\title{
Large-Momentum Effective Theory
}

Xiangdong J因

Maryland Center for Fundamental Physics,

Department of Physics,

University of Maryland,

College Park, Maryland 20742,

USA

Tsung-Dao Lee Institute,

Shanghai Jiao Tong University,

Shanghai, 200240,

China

Yizhuang Liu团

Tsung-Dao Lee Institute,

Shanghai Jiao Tong University,

Shanghai, 200240,

China

Institut fur Theoretische Physik,

Universitat Regensburg,

D-93040 Regensburg,

Germany

Institute of Theoretical Physics,

Jagiellonian University, 30-348 Kraków,

Poland

Yu-Sheng Liu用

Tsung-Dao Lee Institute,

Shanghai Jiao Tong University,

Shanghai, 200240,

China

Jian-Hui Zhang\$

Center of Advanced Quantum Studies,

Department of Physics,

Beijing Normal University, Beijing 100875,

China

Yong Zhad

Physics Department,

Brookhaven National Laboratory Bldg. 510A,

Upton, NY 11973,

USA

Physics Division,

Argonne National Laboratory,

Lemont, IL 60439,

USA

(Dated: April 27, 2021)

Since the parton model was introduced by Feynman more than fifty years ago, we have learned much about the partonic structure of the proton through a large body of highenergy experimental data and dedicated global fits. However, calculating the partonic observables such as parton distribution function (PDFs) from the fundamental theory of strong interactions, QCD, has made limited progress. Recently, the authors have advocated a formalism, large-momentum effective theory (LaMET), through which one can extract parton physics from the properties of the proton travelling at a moderate boost-factor, e.g., $\gamma \sim(2-5)$. The key observation behind this approach is that Lorentz symmetry allows the standard formalism of partons in terms of light-front operators to be replaced by an equivalent one with large-momentum states and time-independent operators of a universality class. With LaMET, the PDFs, generalized PDFs or GPDs, transverse-momentum-dependent PDFs, and light-front wave functions can all be extracted in principle from lattice simulations of QCD (or other non-perturbative methods) 
through standard effective field theory matching and running. Future lattice QCD calculations with exa-scale computational facilities can help to understand the experimental data related to the hadronic structure, including those from the upcoming Electron-Ion Colliders dedicated to exploring the partonic landscape of the proton. Here we review the progress made in the past few years in development of the LaMET formalism and its applications, particularly on the demonstration of its effectiveness from initial lattice QCD simulations.

\section{CONTENTS}

I. Introduction

A. Partons through Infinite-Momentum States

B. Partons through Light-Front Correlators

C. Other Approaches to Parton Structure

II. Large-Momentum Effective Theory

A. Structure of the Proton at Finite Momentum

B. Momentum Renormalization Group

C. Effective Field Theory Matching to PDFs

D. Recipe for Parton Physics in LaMET

E. Universality

III. Renormalization and Matching for PDFs

A. Renormalization of Nonlocal Wilson-Line Operators 1. Renormalization of nonlocal quark operators

2. Renormalization of nonlocal gluon operators

B. Factorization of Quasi-PDFs

C. Coordinate-Space Factorization of Bilinear Operators

D. Nonperturbative Renormalization and Matching

1. Wilson-line mass-subtraction scheme

2. RI/MOM scheme

3. Ratio scheme

4. Hybrid scheme

E. Total Gluon Helicity $\Delta G$ and Transversity PDF

IV. Generalized Collinear Parton Observables

A. Generalized Parton Distributions

B. Hadronic Distribution Amplitudes

C. Higher-Twist Distributions

1. Higher-twist collinear-parton observables

2. Higher-twist contributions to quasi-PDFs

D. Orbital Angular Momemntum of Partons in the Proton

V. Transverse-Momentum Dependent PDFs

A. Introduction to TMDPDFs and Rapidity Divergence

B. Lattice Quasi-TMDPDFs and Matching 40

C. Off-light-cone Soft Function

D. Light-Front Wave-Function Amplitudes And Soft Function from Meson Form Factor

VI. Lattice Parton Physics with LaMET

A. Special Considerations for Lattice Calculations

1. Challenges due to large momentum

2. Considerations for lattice setup

B. Non-Singlet PDFs

1. Proton

2. Pion

\footnotetext{
* xji@umd.edu

$\dagger$ yizhuang.liu@sjtu.edu.cn

$\ddagger$ mestelqure@gmail.com

$\S$ zhangjianhui@bnu.edu.cn

ฯ yong.zhao@anl.gov
}

C. Gluon Helicity and Other Collinear Parton Properties

1. Total gluon helicity 53

2. Gluon PDF 53

3. DA 54

4. GPD 54

5. Higher-twist PDF 55

D. TMDs 55

1. Pre-LaMET study - ratio of lattice correlators 55

2. Quasi-TMDPDF and Collins-Soper kernel 55

3. Soft function

VII. Conclusion and Outlook

Acknowledgments

Appendix A: Acronyms, abbreviations and terminologies 58

Appendix B: Conventions

60

References

\section{INTRODUCTION}

The proton and neutron, collectively called the nucleon, are the basic building blocks of visible matter in the universe today. Ever since they were discovered in laboratories nearly a century ago (Chadwick, 1932; Rutherford, 1919), their fundamental properties have been vigorously explored: from the determination of the spin through the specific heat of liquid hydrogen (Dennison, 1927), to the measurement of the magnetic moments (Estermann et al., 1933), and the extraction of their electromagnetic sizes through elastic electron scattering (Hofstadter, 1956). The most revealing discovery, however, came from the electron deep-inelastic scattering (DIS) on the proton and nuclei at Stanford Linear Accelerator Center (SLAC) in the late 1960s, in which the constituents of the proton and neutron, quarks (and later gluons), were discovered (Bloom et al., 1969). Soon after, quantum chromodynamics (QCD), a quantum field theory (QFT) based on "color" $\mathrm{SU}(3)$ gauge symmetry, was established as the fundamental theory of strong interactions (Fritzsch et al., 1973; Gross and Wilczek, 1973; Politzer, 1973), and of the internal structure of the nucleon as well (Thomas and Weise, 2001).

During the last fifty years, significant progress has been made in understanding the nucleon's internal structure in both experiment and theory. Multiple experimental facilities have been built to study high-energy collisions involving protons and nuclei, from which a large amount 
of experimental data has been accumulated. Based on the QCD factorization theorems (Collins, 2011a), derived from perturbative QCD analyses beyond Feynman's parton model (Feynman, 1972), the parton distribution functions (PDFs), which characterize the longitudinal momentum distributions of quarks and gluons in hadrons moving at infinite momentum, have been obtained from global fits to these data (Ball et al., 2017; Gao et al., 2018; Harland-Lang et al., 2015; Hou et al., 2019). A recent result of the phenomenological proton PDFs is shown in Fig. 1 where $x$ is the momentum fraction of the proton carried by partons. The PDFs provide a comprehensive description of the quark and gluon content of the nucleon. On the theoretical frontier, the Euclidean path-integral formalism of $\mathrm{QCD}$, combined with the lattice regularization and Monte Carlo simulations (Wilson, 1974), has offered a systematic way of performing $a b i n i$ tio calculations of non-perturbative strong interactions. The rapid rise in computational power and development of intelligent numerical algorithms have made such a lattice QCD approach extremely successful in computing hadron spectroscopy, the strong coupling, hadronic form factors, etc., and even scattering phase shifts (Aoki et al., 2020; Briceno et al., 2018; Tanabashi et al., 2018).

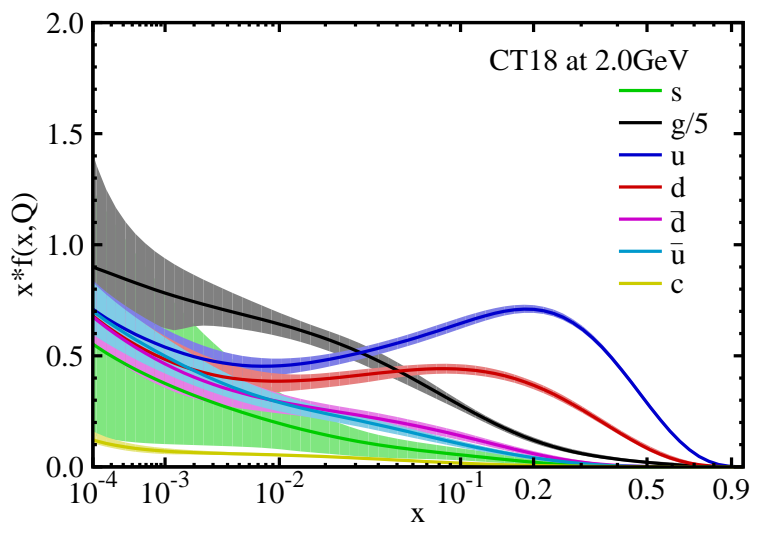

FIG. 1: Phenomenological parton distributions obtained by the CTEQ-TEA collaboration (CT18) from fits to global high-energy scattering data (Hou et al., 2019), where $0 \leq x \leq 1$ is the fraction of the proton's infinite momentum carried in a parton.

Despite these impressive achievements, we have not been able to systematically explain the partonic structure of the proton from first principles, or more explicitly, we have not made fundamental progress in computing the quark and gluon distributions starting from the QCD Lagrangian (see Sec. I.C for a brief summary). There is actually a good reason behind it: The standard formulation of parton physics in the textbooks (Collins, 2011a; Sterman, 1993) is accomplished through the dynamical correlators of quark and gluon fields on the light-front (LF) defined by $t-z=$ const., which has the important feature of being independent of the proton's momentum. On the other hand, lattice QCD is formulated in the Euclidean space with imaginary time, and cannot be used to directly calculate the dynamical correlations that depend on real time. The standard lattice approach to parton physics has been to calculate the lower moments of parton distributions, which are matrix elements of local operators (Lin et al., 2018c). However, the limitations to the first few moments prohibit practitioners from reproducing reliably the $x$-dependent structure as shown in Fig. 1, other than fitting model functional forms. Over the years, Hamiltonian diagonalization in LF quantization (LFQ) (Brodskv et al., 1998) and Schwinger-Dyson equations (Maris and Roberts, 2003) have been proposed to solve the nucleon structure as Minkowskian approaches. Although significant advances have been made phenomenologically, a systematic approximation to calculate the nucleon PDFs is still missing.

A few years ago, some of the present authors proposed a general approach to calculate $x$-dependent parton distributions based on Feynman's original idea about partons: They are the infinite-momentum limit of static properties of the proton at large momentum, and therefore are intrinsically Euclidean quantities accessible through lattice QCD (Ji, 2013, 2014; Ji et al., 2013b). According to this, parton physics in an intermediate range of $x_{\min } \sim 0.1<x<x_{\max } \sim 0.9$ can be calculated from the physical properties of the proton at a moderately-large momentum, e.g., with a Lorentz boost factor $\gamma=2-5$. The theory has been named as largemomentum effective theory (LaMET) because a rigorous connection between the infinite-momentum frame (IMF) partons and quarks and gluons at a finite momentum requires proper account of the ultraviolet (UV) modes with large momentum in effective field theory (EFT) and systematic power counting.

The basic principle for LaMET comes from an implicit observation in the naive parton model: The structure of the proton is approximately independent of its momentum so long as it is much larger than a typical stronginteraction scale $\Lambda_{\mathrm{QCD}}$, or its mass. For example, the quark momentum distribution at moderate $x$ in the proton at $P=|\vec{P}|=5 \mathrm{GeV}$ is not very different from that at $P=50 \mathrm{GeV}$ or $P=5 \mathrm{TeV}$. One might call this phenomenon large-momentum symmetry, the nature of which is similar to that of the electronic structure of the hydrogen atom is not sensitive to the proton mass, so long as it is much larger than that of the electron. The asymptotic behavior of the proton structure might be controlled by an expansion in $\Lambda_{\mathrm{QCD}} / P$, but a justification would require a better understanding of the underlying dynamics. Assuming this, Feynman replaced the protons probed at large but finite momenta in high-energy scattering with the one at infinite momentum $P=\infty$, corresponding to the leading term in the $\Lambda_{\mathrm{QCD}} / P$ expansion, and therefore the idealized concepts of the proton in the IMF and its constituents - partons - were born. 
In QFTs, however, the existence of the $P=\infty$ limit depends on their UV behavior. In general, the infinitemomentum limit does not commute with the UV cut-off limit $\Lambda_{\mathrm{UV}} \rightarrow \infty$. While the physical limit is $\left(\Lambda_{\mathrm{UV}} \gg\right.$ $P) \rightarrow \infty$, the parton model and subsequent QCD factorization theorems use $\left(P \gg \Lambda_{\mathrm{UV}}\right) \rightarrow \infty$, keeping all PDFs with the finite support $|x| \leq 1$ where negative $x$ is for antiquarks. Thus partons are an idealized concept which does not exist in the real world. Fortunately, because of asymptotic freedom, the above differences can be calculated in perturbative QCD. Therefore, LaMET is an effective theory of partons, which uses the ordinary field theoretical calculations $\left(\Lambda_{\mathrm{UV}} \gg P\right) \rightarrow \infty$ and systematically takes into account non-commuting $P \rightarrow \infty$ limits through EFT matching and running and finite $P$ effects by power corrections. Thus, the PDFs defined in the IMF or on the LF can be accessed at moderate $x$ from the structure calculations at $P \sim$ a few $\mathrm{GeVs}$.

The first application of LaMET was to the total gluon helicity $\Delta G$ in the polarized proton, a quantity of significant experimental interest at the polarized RHIC (Bunce et al., 2000), but not within theoretical reach for many years. In (Ji et al., 2013b), we have shown that from a large-momentum matrix element of the gluon spin operator in a physical gauge, $\Delta G$ can be obtained through an EFT matching. Following this success, LaMET was applied to the collinear quark PDFs (Ji, 2013). This latter application has generated considerable theoretical as well as numerical activities, particularly for the flavor non-singlet $u-d$ distributions in the proton and other hadrons. A general LaMET framework was subsequently introduced in (Ji, 2014). More recently, the approach has been extended to the gluons as well (Li et al., 2019; Zhang et al., 2019b). Therefore, the PDFs can now be computed directly in lattice QCD at specific Feynman variable $x$, without using LFQ. Besides, the partonic landscape of the proton is extremely rich, and LaMET holds the promise of computing parton physics beyond the collinear PDFs.

In recent years, tremendous progress has been made in formulating new parton observables for the proton. In particular, two parallel concepts have been developed in characterizing the transverse structure of the proton. The first is the generalized parton distributions (GPDs) (Ji, 1997b; Müller et al., 1994; Radyushkin, 1999). The GPDs combine the features of the proton's elastic form factors, which provide the transverse-space density of partons (Miller, 2007), and Feynman PDFs, and interpolate them. Given the joint longitudinalmomentum and transverse-space distributions, one can construct the orbital angular momentum (OAM) of partons, among others (Ji, 1997b). In general, the GPDs can be used to generate momentum-dissected transverse space images of the proton (Burkardt, 2000). A new class of experimental processes, deeply-virtual exclusive processes (DVEP), including deeply-virtual Compton scat- tering (DVCS) in which the final state is a diffractive real photon plus a recoiling proton, has been found to measure them (Ji, 1997a, $\mathrm{b})$. The second concept is the transverse-momentum-dependent (TMD) PDFs (or TMDPDFs), in which the parton's transverse momentum is explicit Collins, 2011a; Collins and Soper, 1981). Much theoretical progress has been made in recent years regarding their proper definitions, factorizations, and spin correlations (Collins and Rogers, 2017, 2013; Echevarría et al., 2013). TMDPDFs can be measured in experimental processes by observing the transverse momentum of the final-state particles.

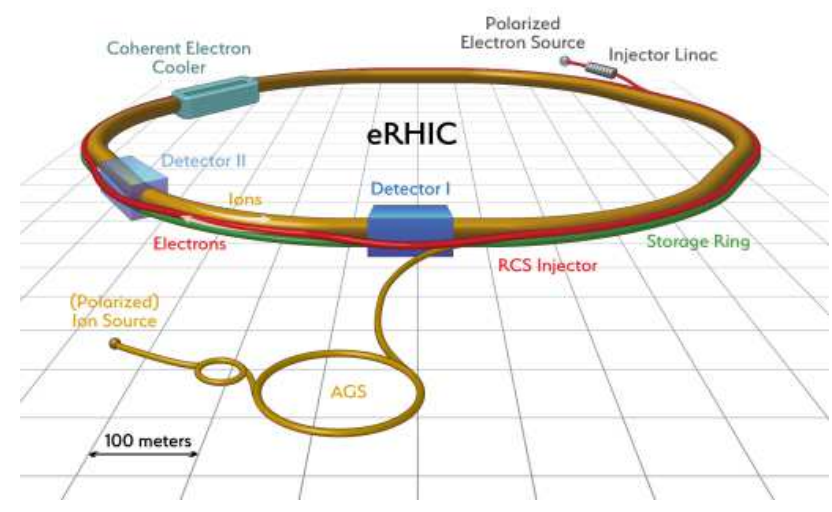

FIG. 2: A realization of Electron-Ion Collider at BNL (figure credit to BNL), which can be used to probe the partonic landscape of the proton.

Over the years, it has gradually become clear that a dedicated experimental facility to fully explore the partonic landscape of the proton is required. To meet this requirement, the US nuclear science community has proposed, to build a high-energy, high-luminosity ElectronIon Collider (EIC) (Aprahamian et al., 2015), which has been recently approved by the US Department of Energy. The new collider accelerates electrons to $10-30 \mathrm{GeV}$ and ions - including the proton and heavy nuclei all the way up to $\mathrm{Pb}$ or $\mathrm{U}$ - up to $100 \mathrm{GeV}$ per nucleon, realizing the center-of-mass collision energy $E_{\mathrm{cm}}$ from 40 to $170 \mathrm{GeV}$. The corresponding electron energy in fixedtarget experiments would be $100 \mathrm{GeV}$ to $10 \mathrm{TeV}$. The beams are polarized, with high-luminosity up to $10^{33-34}$ collisions $/\left(\mathrm{cm}^{2} \cdot \mathrm{s}\right)$, which are critical for studying exclusive processes such as DVCS. The kinematic range of the collisions covers the Bjorken $x_{B}$ (which coincides with the parton momentum fraction $x$ in the naive parton model to be discussed in the next section) down to sub- $10^{-4}$, and $Q^{2}$ as high as $10^{4} \mathrm{GeV}^{2}$. Much of the EIC science has been discussed in a dedicated study (Accardi et al., 2016b).

Of course, the EIC and lattice QCD efforts will not stop at the precision parton physics of the proton. More importantly, we need to develop ways or languages to describe the nucleon as a strongly-coupled relativistic quantum system, in much the same way as we understand, for 
example, the quantum Hall effects in condensed matter physics. Without a deep understanding of the mechanisms of strongly-coupled QCD physics, we cannot claim a fundamental understanding of the structure of the proton and neutron, in particular, the origin of their mass and spin. This is one of the most challenging goals facing the standard model of particle and nuclear physics today.

This review is to systematically expose the idea, formalism, and results of the LaMET approach to parton physics. We do not claim to be entirely complete because the field is rapidly developing. References in the related fields are not meant to be complete either, and we apologize for any important omissions. Closely-related reviews on lattice parton physics can be found in (Cichy and Constantinou, 2019; Zhao, 2019). There have been studies on the effectiveness of LaMET in various models (Bhattacharya et al., 2019a,b; Broniowski and Ruiz Arriola, 2017, 2018; Del Debbio et al., 2020b; Gamberg et al., 2015; Hobbs, 2018; Ji et al., 2019c; Jia and Xiong, 2016; Kock et al., 2020; Ma et al., 2019; Nam, 2017; Radyushkin, 2017d; Son et al., 2019; Xu et al., 2018), some of which we will mention in the following for illustrative purposes. There have been also papers questioning the validity of LaMET method (Carlson and Freid, 2017; Rossi and Testa, 2017, 2018) and some got clarified later in the literature (Briceño et al., 2017; Ji et al., 2017b; Radyushkin, 2019c), We will not discuss them here and interested readers may refer to the above references. We have used proton in most places in the text to emphasize its importance in nuclear and particle physics. However, the discussions apply equally to the neutron and other hadrons as well.

The plan for the presentation of this review is as follows. In the remainder of the Introduction, we explain the nature of parton physics as an effective description of the internal structure of the proton at large momentum, as well as other existing methods in the literature for solving the parton structure. In Sec. II], we introduce the LaMET method starting from momentum renormalization group equation (RGE) of physical observables in a moving hadron, followed by the matching between momentum distributions and PDFs. We then formulate an EFT expansion to compute parton physics from theoretical methods suitable for the structure of a large-momentum proton. In Sec. III, we discuss some important details for collinear PDFs: renormalization of the nonlocal operators, particularly power divergences in lattice regularization, and matching to all orders in perturbation theory. Sec. IV is devoted to applications to general collinear parton observables including GPDs, parton distribution amplitudes and higher-order parton correlations. We also discuss applications for the OAM of the partons in a polarized proton. In Sec. V], we consider the application to TMDPDFs, a new class of parton observables. We study matching of the quasi-TMDPDFs to the physical ones, and explore the lattice calculation of the soft function. Finally, Sec. VI summarizes the recent lattice calculations relevant to the LaMET applications, and the conclusion is given in Sec. VII] The review is completed with an Appendix with a list of acronyms and glossaries, as well as notations and conventions.

\section{A. Partons through Infinite-Momentum States}

Although partons have become a ubiquitous language for high-energy scattering, their role as effective degrees of freedom of QCD for describing the internal structure of the nucleon is less emphasized in the literature. In applications within QCD factorization theorems, they arefollowing Feynman - objects arising from the limit of infinite momentum, with the potential UV divergences regulated and renormalized after the limit. Thus, the partons are an idealized concept, referring to the quark and gluon Fock components of the nucleon or other hadrons only in the context of IMF and LF gauge $A^{+}=\left(A^{0}+A^{z}\right) / \sqrt{2}=$ 0 . They are in the same category of concepts as the infinitely-heavy quark in heavy-quark effective field theory (HQET) (Manohar and Wise, 2000). To motivate LaMET, it is important to understand this origin and nature of partons.

Built from the knowledge of electron scattering in non-relativistic systems (atoms and molecules) (West, 1975), Feynman introduced the naive parton model to describe deep-inelastic scattering (DIS) on the proton, and to explain the observed phenomenon of Bjorken scaling (Bjorken and Paschos, 1969; Fevnman, 1972, 1969).

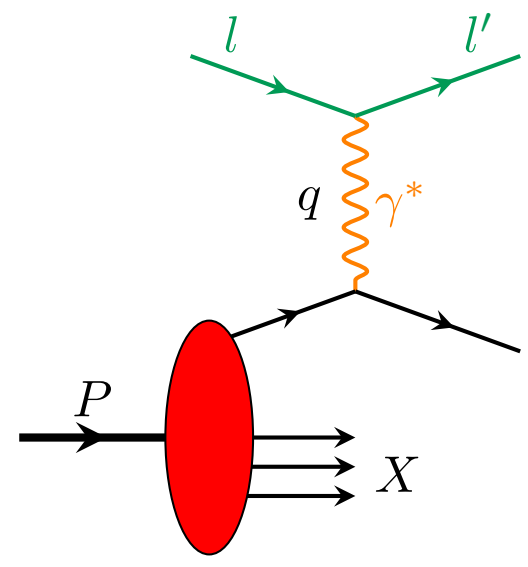

FIG. 3: Deep-inelastic scattering in which partons are probed in the proton.

Shown in Fig. 3 is the DIS process in which a virtual photon with large momentum $q^{\mu}$ is absorbed by a proton of momentum $P^{\mu}$ and mass $M$. The invariant variables are $Q^{2}=-q^{\mu} q_{\mu}$ and $P \cdot q=M \nu$, and Bjorken $x_{B}=Q^{2} /(2 P \cdot q)$ fixed in the scaling (or Bjorken) limit $Q^{2} \rightarrow \infty, P \cdot q \rightarrow \infty$. The inclusive DIS cross section can 
be factored into a product of leptonic and hadronic tensors, where the former is associated with the electromagnetic current of the lepton, while the latter contains all information about the electromagnetic interaction with the target proton.

To learn about the proton structure, it is best to consider the scattering in the Breit frame where

$$
\begin{aligned}
q^{\mu} & =(0,0,0,-Q), \\
P^{\mu} & =\left(\sqrt{\frac{Q^{2}}{4 x_{B}^{2}}+M^{2}}, 0,0, \frac{Q}{2 x_{B}}\right),
\end{aligned}
$$

and the virtual photon has zero energy. The probe is sensitive only to the spatial structure as in non-relativistic electron scattering. However, relativity now constrains the proton to move at a large momentum $P^{z}=Q /\left(2 x_{B}\right)$ with boost factor $\gamma=Q /\left(2 x_{B} M\right)$, which approaches $P^{z}=\infty$ in the Bjorken limit.

Feynman made intuitive assumptions about the proton structure and scattering mechanism, without QFT subtleties (Feynman, 1972): The proton structure at different large $P^{z}$ should be similar, and can be approximated by that at $P^{z}=\infty$, or in the IMF. The interactions between constituents (partons) are infinitely time-dilated, and the wave function configurations are frozen. The proton in high-energy scattering can be seen as being made of non-interacting partons, each with a longitudinal momentum $x P^{z}$ with $0<x<1$.

The internal structure of non-relativistic systems is independent of their overall momentum. However, relativistic systems is different as they least experience the Lorentz contraction. The structures of such systems are inextricably mixed with the overall motion, and their dependence on the external momentum is a dynamical problem. On the other hand, if the internal structure depends on a particular hadron scale $\Lambda_{\mathrm{QCD}}$, the protons at all large-momentum with $P^{z} \gg \Lambda_{\mathrm{QCD}}$ have a similar structure, corresponding to the $P^{z} \rightarrow \infty$ limit. This means that if $f\left(k^{z}, P^{z}\right)$ is the constituent momentum- $k^{z}$ distribution in a proton of momentum $P^{z}$, it might be analytical at $P^{z}=\infty$ and admits Taylor series expansions in $1 / P^{z}$,

$$
f\left(k^{z}, P^{z}\right)=f(x)+f_{2}(x)\left(\Lambda_{\mathrm{QCD}} / P^{z}\right)^{2}+\ldots,
$$

where $x=k^{z} / P^{z}$. If so, one may find a large-momentum symmetry of the proton properties up to power corrections $\mathcal{O}\left(1 / P^{z}\right)$ (we omit the upper index $z$ sometimes for simplicity), and $f(x)$ is the parton distribution.

The above picture can be shown to hold in certain simple QFT models, where the dynamical frame dependence of wave functions for composite systems can be studied straightforwardly. There are many interesting examples of two-dimensional systems, for which solutions can be found. One of the much studied cases is the large $N_{c}$ QCD, also called 't Hooft model ('t Hooft, 1974), in which the bound states have a well-defined largemomentum limit. The wave functions can be expanded in $1 / P$, with the corrections starting from $(1 / P)^{2}$. The momenta of the constituents, $k$ and $P-k$, scale in this limit. When plotted as a function of $x=k / P$, the change in the wave function with the magnitude of the momentum can be found in Figs. 8-11 in (Jia et al., 2017). This is the type of example in which Feynman's intuition applies.

However, such a intuition fails in many $3+1$ dimensional QFTs, such as QCD. When a bound state travels at increasingly large momentum, more and more highmomentum modes of a field theory are needed to build up its internal structure. Lorentz contraction indicates that the range of constituent momentum important for the structure also increases. If these high-momentum modes do not decouple effectively from the low-momentum ones, large logarithms of the form $\ln P$, will develop in the structural quantities. Hence a singularity (cut) at $P=\infty$ can exist in these theories, making $P \rightarrow \infty$ limit illdefined and the large momentum expansion impossible. This situation is intimately related to UV properties of the theories, for which the limits of taking the UV cutoff $\Lambda_{\mathrm{UV}} \rightarrow \infty$ and $P \rightarrow \infty$ do not commute. While the physically-relevant one is $\left(\Lambda_{\mathrm{UV}} \gg P\right) \rightarrow \infty$, partons in QCD factorizations are obtained in the other limit $\left(\Lambda_{\mathrm{UV}} \ll P\right) \rightarrow \infty$ when the UV divergences are ignored. Thus one can formally write the parton distribution as

$$
f(x)=\int \frac{d \lambda}{2 \pi} e^{i x \lambda}\left\langle P^{z}=\infty\left|\psi^{\dagger}(z) \psi(0)\right| P^{z}=\infty\right\rangle,
$$

where $\lambda=\lim _{P^{z} \rightarrow \infty, z \rightarrow 0}\left(z P^{z}\right)$, and $\psi$ is a quantum field.

Historically, the IMF limit of field theories has been studied first at the level of diagrammatic rules for perturbation theory (Weinberg, 1966). It was found that taking $P \rightarrow \infty$ by ignoring the UV divergences considerably simplifies the perturbation theory rules: Many timeordered diagrams vanish and only few have finite contributions. Moreover, scattering in this limit resembles that in non-relativistic quantum mechanics, and the wave function description becomes useful. The Fock states define the partons which have the proper kinematic support $(0<x<1)$. After the limit is taken, all physical quantities are now independent of $P$, and large-momentum symmetry is exact before UV divergences are regulated. Therefore, it is the "naive" limit, $\Lambda_{\mathrm{UV}} \ll P \rightarrow \infty$, that corresponds to Feynman's naive parton model.

In the standard QCD study of high-energy scattering, the above concept of partons as effective degrees of freedom has been used implicitly. The PDFs are defined in terms of the naive $P=\infty$ limit, and are used to match the experimental cross sections, resulting in QCD factorization theorems (Collins, 2011a). 


\section{B. Partons through Light-Front Correlators}

In the literature and textbooks, parton distributions are not traditionally represented in terms of the Euclidean matrix elements as in Eq. (3). Rather, they are represented by the so-called LF correlators of quantum fields ("operator formalism") (Brodsky et al., 1998; Collins, 2011a). A more explicit formulation in terms of collinear quantum fields and effective lagrangian is made in the soft-collinear effective theory (SCET) (Bauer et al., 2001, 2002; Bauer and Stewart, 2001).

There is a physical way to see that the parton description of high-energy scattering results in the light-front correlations. Consider DIS in the rest frame of the proton, where the virtual photon has momentum

$$
q^{\mu}=\left(\nu, 0,0,-\sqrt{\nu^{2}+2 x_{B} M \nu}\right) .
$$

In the Bjorken limit $\nu \rightarrow \infty$, although the invariant mass $Q$ of the photon goes to infinity, the photon momentum becomes actually light-like in the sense that it approaches the light front. Therefore, in inclusive DIS cross section, the separation of the two electromagnetic currents in the hadronic tensor, which is Fourier conjugate to the photon momentum, also approaches the light-cone direction.

Thus, it appears natural that all the structural physics of the proton in the IMF can also be expressed in terms of time-dependent LF correlators or correlations of quantum fields on the LF. Formally, this is simple to see if one writes

$$
|P \rightarrow \infty\rangle=U\left(\Lambda_{\infty}\right)|P=0\rangle
$$

The boost operator $U\left(\Lambda_{\infty}\right)$ can be applied to the static nonlocal operators in the ordinary momentum distributions. In doing so, all static correlations become light-cone ones. The boost process is then similar to shifting the Hamiltonian evolution in quantum mechanics from Schrödinger to Heisenberg picture where timedependence is now in the operators.

To express light-cone correlations, it is convenient to introduce two conjugate light-like (or light-cone) vectors, $p^{\mu}=(\Lambda, 0,0, \Lambda)$ and $n^{\mu}=(1 / 2 \Lambda, 0,0,-1 / 2 \Lambda)$, with the following properties, $n^{2}=p^{2}=0$, and $n \cdot p=1$, where $\Lambda$ is a parameter. Then any four-vector can be expanded as,

$$
k^{\mu}=k \cdot n p^{\mu}+k \cdot p n^{\mu}+k_{\perp}^{\mu} .
$$

In particular, the momentum $P^{\mu}$ of a proton moving in the $z$-direction can be expressed as

$$
P^{\mu}=p^{\mu}+\left(M^{2} / 2\right) n^{\mu},
$$

where $M$ is the proton mass.
Using the above notation, one can express the unpolarized quark distribution in the proton as (Collins, 2011a),

$$
q(x)=\frac{1}{2} \int \frac{d \lambda}{2 \pi} e^{i \lambda x}\langle P|\bar{\psi}(0) \not h W(0, \lambda n) \psi(\lambda n)| P\rangle_{c}
$$

where $\psi$ is the quark field and $W$ is a gauge-link defined as

$$
\begin{aligned}
& W\left(x_{2}, x_{1}\right)= \\
& \mathcal{P} \exp \left[-i g \int_{0}^{1} d t\left(x_{2}-x_{1}\right)_{\mu} A^{\mu}\left(x_{1}+\left(x_{2}-x_{1}\right) t\right)\right]
\end{aligned}
$$

to ensure gauge invariance with $\mathcal{P}$ denoting the path ordering. $c$ indicates the connected contributions only, and will be suppressed in the rest of this work. It is a property of gauge theories in which the charge fields are not gauge-invariant, and the physical distributions must include a beam of collinear gauge particles. Note that the above expression is true for any momentum $P$ (a residual momentum symmetry), in particular, in the rest frame of the nucleon. The $x$-support of the above distribution is $[-1,1]$. For negative $x$, one defines the antiquark distribution with $-q(-x) \equiv \bar{q}(x)$. The above expression has been more familiar in the literature than Feynman's original formulation of PDFs. In the single quark target, one finds $q(x)=\delta(x-1)$.

To expose the partons in the above equation, one can follow the QCD light-front quantitzation Chang and Ma, 1969; Drell and Yan, 1971; Kogut and Soper, 1970), suggested by Dirac in 1949 (Dirad, 1949). In LFQ (Brodsky et al., 1998), one defines the LF coordinates,

$$
\xi^{ \pm}=\left(\xi^{0} \pm \xi^{3}\right) / \sqrt{2}
$$

where $\xi^{+}$is the LF "time", and $\xi^{-}$is the LF "spatial coordinate". And any four-vector $A^{\mu}$ will be now written as $\left(A^{+}, A^{-}, \vec{A}_{\perp}\right)$. Dynamical degrees of freedom are defined on the $\xi^{+}=0$ plane with arbitrary $\xi^{-}$and $\vec{\xi}_{\perp}$, with conjugate momentum $k^{+}$and $\vec{k}_{\perp}$. Dynamics is generated by the light-cone Hamiltonian $H_{\mathrm{LC}}=P^{-}$. For a free particle with three-momentum $\left(k^{+}, \vec{k}_{\perp}\right)$ and mass $m$, the on-shell LF energy is $k^{-}=\left(\vec{k}_{\perp}^{2}+m^{2}\right) /\left(2 k^{+}\right)$.

For QCD, one can define the Dirac matrices $\gamma^{ \pm}=$ $\left(\gamma^{0} \pm \gamma^{3}\right) / \sqrt{2}$, and the projection operators for the quark fields as $P_{ \pm}=(1 / 2) \gamma^{\mp} \gamma^{ \pm}$, so that any $\psi$ can be decomposed into $\psi=\psi_{+}+\psi_{-}$with $\psi_{ \pm}=P_{ \pm} \psi$, where $\psi_{+}$is considered as a dynamical degree of freedom. For the gauge field, $A^{+}$is fixed by the LF gauge $A^{+}=0 . A_{\perp}$ are dynamical degrees of freedom. $\psi_{-}$and $A^{-}$are dependent variables, which can be expressed in terms of $\psi_{+}$and $A_{\perp}$ using equations of motion (Kogut and Soper, 1970).

The physics of the LF correlations becomes manifest if 
one introduces the canonical expansion,

$$
\begin{aligned}
& \psi_{+}\left(\xi^{+}=0, \xi^{-}, \vec{\xi}_{\perp}\right)=\int \frac{d^{2} k_{\perp}}{(2 \pi)^{3}} \frac{d k^{+}}{2 k^{+}} \sum_{\sigma}\left[b_{\sigma}(k) u(k, \sigma)\right. \\
& \left.\times e^{-i\left(k^{+} \xi^{-}-\vec{k}_{\perp} \cdot \vec{\xi}_{\perp}\right)}+d_{\sigma}^{\dagger}(k) v(k, \sigma) e^{i\left(k^{+} \xi^{-}-\vec{k}_{\perp} \cdot \vec{\xi}_{\perp}\right)}\right],
\end{aligned}
$$

where $b^{\dagger}(k)$ and $d^{\dagger}(k)(b(k)$ and $d(k))$ are quark and antiquark creation (annihilation) operators, respectively. $\sigma$ is the light-cone helicity of the quarks which can take $+1 / 2$ or $-1 / 2$. Covariant normalization is adopted for the particle states and the creation and annihilation operators, i.e.,

$$
\begin{aligned}
& \left\{b_{\sigma}(k), b_{\sigma^{\prime}}^{\dagger}\left(k^{\prime}\right)\right\}=\left\{d_{\sigma}(k), d_{\sigma^{\prime}}^{\dagger}\left(k^{\prime}\right)\right\} \\
& =(2 \pi)^{3} \delta_{\sigma \sigma^{\prime}} 2 k^{+} \delta\left(k^{+}-k^{\prime+}\right) \delta^{(2)}\left(\vec{k}_{\perp}-\vec{k}_{\perp}^{\prime}\right) .
\end{aligned}
$$

Substituting the above expansion into Eq. (8), one finds the quark distribution as

$$
q(x)=\frac{1}{2 x} \sum_{\sigma} \int \frac{d^{2} \vec{k}_{\perp}}{(2 \pi)^{3}}\left\langle P\left|b_{\sigma}^{\dagger}\left(x, \vec{k}_{\perp}\right) b_{\sigma}\left(x, \vec{k}_{\perp}\right)\right| P\right\rangle /\langle P \mid P\rangle
$$

for $x>0$, and similarly for $x<0$ for which one gets the antiquark distribution. The factor $1 / x$ comes from the normalization of the creation and annihilation operators. The matrix element above should be interpreted as the matrix element in a wave packet state, in the limit of a state of definite momentum (Collins, 2011a). This way, one recovers the physical meaning of PDFs in the LF correlator (operator) formalism.

\section{Other Approaches to Parton Structure}

Calculating the partonic structure of the hadrons from QCD has always been an important goal in hadronic physics. There have been two main approaches apart from various phenomenology and models: light-front quantization and lattice QCD. Here the authors give a very brief review on LFQ and lattice approaches that are different from the main subject of this review.

Although LFQ explicitly uses the parton degrees of freedom, it has not been very successful in practical calculations. First of all, LF perturbation theory, like the standard Hamiltonian perturbation theory, breaks Lorentz symmetry manifestly and requires a sophisticated renormalization scheme to restore it. A potential renormalization scheme must deal with the longrange correlations in the $\xi^{-}$direction which require functional dependence on the renormalization counterterms (Wilson et al., 1994). Thus LF perturbation theory has not been used for any calculations beyond one loop, except for the two-loop anomalous magnetic moment in QED (Langnau and Burkardt, 1993). In fact, the common wisdom of using dimensional regularization
(DR) for the transverse integrals and cut-off regularization for the longitudinal one has not been proven useful for multi-loop calculations, although it has been successfully used to derive the BFKL evolution by Mueller from the quarkonium wave functions (Mueller, 1994a).

The enthusiasm for using LFQ in QCD is not about perturbation theory, but to solve the hadron states. Discretized LFQ was proposed in (Pauli and Brodsky, 1985) to make practical calculations for the bound state problems. This non-perturbative method turns out to be successful for models in $1+1$ dimension, such as the Schwinger model (Harada et al., 1996; McCartor, 1994), the 1+1 QCD (Burkardt, 1989; Srivastava and Brodsky, 2001), the $1+1 \phi^{4}$ theory (Harindranath and Vary, 1987) and the sine-Gordon model (Burkardt, 1993). For $3+1$ dimensional theories, simple approximations have been considered, like the Tamm-Dancoff approximation (Perry et al., 1990). For QCD itself, one again has to use severe truncations in the number of Fock states. Some recent works of this type include (Jia and Vary, 2019; Lan et al., 2019; Vary et al., 2010). However, to derive a fully-renormalized hamiltonian is difficult and moreover, there has been no demonstration so far that the Fock-space truncation actually converges (Wilson et al., 1994). Therefore a systematic approximation for QCD bound states in LFQ has yet to be found.

Given the rapid development in lattice QCD, it is natural to use it to compute parton physics. However, simulating real-time evolution directly is numerically challenging, which runs into the so-called sign problem or more generally NP-hard problem. Over the years, a number of methods have been proposed previously to indirectly calculate the PDFs, which includes well-studied moment methods, hadronic tensor and Compton amplitude method, coordinate space factorization, etc. These approaches calculate lattice observables that can be related to the PDFs/structure functions through OPE or the dispersion relation, and thus can be used to probe certain information on the partonic structure of hadrons. However, their aims are mainly to get the lower moments of PDFs and/or segments of certain coordinate correlations, not directly in parton degrees of freedom.

The most-adopted approach on the lattice has been to calculate the moments of PDFs as the matrix elements of local operators (Kronfeld and Photiadis, 1985; Martinelli and Sachrajda, 1987). In the moments approach, one starts with the so-called twist-two operators (Christ et al., 1972),

$$
O^{\mu_{1} \ldots \mu_{n}}=\bar{\psi} \gamma^{\left(\mu_{1}\right.} i D^{\mu_{2}} \ldots i D^{\left.\mu_{n}\right)} \psi-\text { trace }
$$

in the quark case, where $\left(\mu_{1} \ldots \mu_{2}\right)$ indicates that all the indices are symmetrized, the trace terms are those with at least one factor of the metric tensor $g^{\mu_{i} \mu_{j}}$ multiplied by operators of dimension $(n+2)$ with $n-2$ Lorentz 
indices, etc. Their matrix elements in the proton state are

$$
\left\langle P\left|O^{\mu_{1} \ldots \mu_{n}}(\mu)\right| P\right\rangle=2 a_{n}(\mu)\left(P^{\mu_{1}} \cdots P^{\mu_{n}}-\text { trace }\right),
$$

and the PDFs are related to the local matrix elements through

$$
\begin{aligned}
a_{n}(\mu) & =\int_{-1}^{1} d x x^{n-1} q\left(x, \mu^{2}\right) \\
& =\int_{0}^{1} x^{n-1}\left[q\left(x, \mu^{2}\right)+(-1)^{n} \bar{q}\left(x, \mu^{2}\right)\right]
\end{aligned}
$$

with $n=1,2, \ldots$. The time-dependent correlation for the PDF in Eq. (8) is recovered by taking all the components as + in Eq. (15),

$$
\left\langle P\left|O^{+\ldots+}(\mu)\right| P\right\rangle=2 a_{n}(\mu) P^{+} \ldots P^{+},
$$

and packaging all the moments into a distribution. Likewise, for the gluon PDF, its moments are again related to the matrix elements of local operators,

$$
O_{g}^{\mu_{1} \ldots \mu_{n}}=-F^{\left(\mu_{1} \alpha\right.} i D^{\mu_{2}} \cdots . i D^{\mu_{n-1}} F_{\alpha}^{\left.\mu_{n}\right)},
$$

with $n=2,4,6, \ldots$.

A large number of lattice QCD calculations of PDF moments have been done so far with various degrees of control in systematics (Lin et al., 2018c), which include discretization errors, physical pion mass, finite volume effects, excited state contaminations, and proper renormalization. Most of the lattice calculations have been focused on the first and second moments, $\langle x\rangle$ (Alexandrou et al., 2017a; Bali et al., 2014; Green et al., 2014), and $\left\langle x^{2}\right\rangle$ (Deka et al., 2009; Dolgov et al., 2002) for the unpolarized distributions, and the zero-th and first moments, $\langle 1\rangle$ (Alexandrou et al., 2019a, 2017a; Chang et al., 2018; Gong et al., 2017), and $\langle x\rangle$ (Abdel-Rehim et al., 2015; Aoki et al., 2010) for the polarized distributions. However, it has been difficult to calculate higher moments, due to power divergences and rapid decay in signals. Nonetheless, moment calculations can provide a useful calibration for any comprehensive lattice approach to PDFs.

To get more information about the PDFs, it was proposed to calculate the hadronic tensor of DIS in Euclidean space, and analytically continue the result to Minkowski space (Liu, 2000, 2016, 2017, 2020; Liu and Dong, 1994; Liu et al., 1999). Since numerical methods for analytical continuation are known to be difficult for precision control (similar to NP-hard or sign problem mentioned earlier), the approach is useful mainly for the nucleon low-lying excitations. It is very challenging to obtain parton physics this way.

A similar approach called "operator product expansion (OPE) without OPE" was suggested in Aglietti et al., 1998; Martinelli, 1999), see also (Capitani et al., 1999a, b;
Dawson et al., 1998). The point is that the Compton amplitude in the non-dispersive region can be calculated in the Euclidean space (Ji and Jung, 2001). Through dispersion relation and Taylor-expansion at $\nu=P \cdot q=0$, one can extract the higher moments of structure functions from the lattice Compton amplitude. The recent works and references for parton structure from this approach can be found in (Chambers et al., 2017; Hannaford-Gunn et al., 2020; Horslev et al., 2020). A similar method has been adopted for Compton amplitude with heavy-light currents (Detmold and Lin, 2006). This approach has been used to calculate the second moment of pion distribution amplitude (Detmold et al., 2020, 2018).

The current-current correlators can also be studied through OPE in the coordinate space without momentum insertion into the currents (Braun and Müller, 2008). The spatial correlation at small distances can be used to calculate higher-moments of distribution amplitudes of the mesons. A number of lattice studies have been performed in (Bali et al., 2019, 2018a; Braun et al., 2015). Similar strategy has been suggested more recently by Qiu et al. (Ma and Qiu, 2018a) for parton distributions, and has been used in lattice simulations (Sufian et al., 2020, 2019). The pseudo-PDF has been proposed based on the equal-time correlation - or the quasi-PDF in Fourier space - used in LaMET (Radyushkin, 2017a, 2019b), and uses a coordinate-space factorization or OPE at small distance as in (Braun and Müller, 2008). Because of its close connection with the quasi-PDF, we will discuss comparisons of the pseudo-PDF data analysis method with that for the quasi-PDF in Sec. III.C.

There have been pioneering studies on moments of the "quasi" quark TMDPDFs on lattice (Engelhardt et al., 2016; Hagler et al., 2009; Musch et al., 2012, 2011; Yoon et al., 2017). The staple-shaped gauge link operators have been used to connect the quark fields separated in the spatial direction to simulate the moments of TMDPDF. The ratios of these moments are presumed independent of the unknown soft function and may be compared with experimental data. However, a rigorous relation of these constructions to the physical moments of TMDPDFs had not been investigated before LaMET, particularly the relationship between large momentum limit and the rapidity cutoff which is an essential ingredient of TMD physics. Comparison of this approach and LaMET will be made in Sec. V.B.

\section{LARGE-MOMENTUM EFFECTIVE THEORY}

As has been explained in Sec. I.A Feynman's partons were motivated from describing the structure of a bound state travelling at large momentum $P$. On the other hand, in QCD factorizations, they appear as effective degrees of freedoms arising in infinite momentum 
limit disregarding UV divergences. Reconciling these two pictures results in large-momentum effective theory (LaMET) for the parton structure of hadrons.

In this section, we start by considering the structure of the proton at finite momentum. We define the ordinary momentum distributions of the constituents, and trying to illustrate their dependence on the proton momentum. We demonstrate that the large- $P$ momentumdependence follow a RGE, similar to the well-known RGE for partons. In Sec. II.C we show that momentum distributions at large $P$, are related to PDFs through a matching between different orders of $P \rightarrow \infty$ and UV cut-off limits. This matching process has a standard EFT explanation: Parton physics or observables can be obtained from an effective theory in which $P \ll \Lambda_{\mathrm{UV}}$ are calculated non-perturbatively in the so-called $\mathcal{P}$ space Messiah, 1979), after "integrating out" degrees of freedom between $P$ and $\infty$ (or $\mathcal{Q}=1-\mathcal{P}$ space) through perturbation theory. Therefore, the LaMET approach to partons is in some sense similar to lattice QCD as an EFT approach for continuum field theories, in which all active degrees of freedom ( $\mathcal{P}$ space) are bounded by $|k| \leq \pi / a$, where $a$ is lattice spacing, whereas those at $|k| \geq \pi / a$ ( $\mathcal{Q}$ space) are taken into account through perturbative coefficients and higher dimensional operators.

In Sec. II.D we outline the formalism of LaMET for a general parton observable. The method can in principle be used also to calculate any LF correlations in terms of large momentum external states (see in particular the application to soft function in Sec. (V). The strategy is also applicable for the components of the LF wave functions. Thus, LaMET offers a practical and systematic way to carry out the program of LFQ. Instead of working with the LF coordinates directly, one uses the instant form of dynamics and large momentum or boost factor $\gamma$ as a regulator for the LF divergences. In a certain sense, the quantization using tilted light-cone coordinates (Lenz et al., 1991) is similar to the spirit of the LaMET approach.

At present, the only systematic approach to solve non-perturbative QCD is lattice field theory (Wilson, 1974). Therefore, a practical implementation of LaMET can be done through lattice calculations. It can also be done with other bound-state methods using Euclidean approaches, such as the instanton liquid model (Schäfer and Shurvak, 1998). While LFQ may provide an attractive physical picture for the proton, the Euclidean equal-time formulation is more practical for carrying out the calculations, and LaMET serves to bridge them.

\section{A. Structure of the Proton at Finite Momentum}

In relativistic theories, the internal structure of a composite system is frame-dependent (we always refer to the total momentum eigenstates), and we are interested in the properties of the proton at a momentum much larger than its rest mass.

We start from the quark momentum density in a fastmoving proton, assuming that it moves in the $z$-direction. A straightforward definition is

$$
N_{P}(\vec{k})=\sum_{\sigma}\left\langle P\left|b_{\sigma}^{\dagger}(\vec{k}) b_{\sigma}(\vec{k})\right| P\right\rangle /\langle P \mid P\rangle,
$$

where the quark helicity, color, and other implicit indices are summed over. This equation should be compared with the parton density in Eq. (13). To make it gauge invariant, it is convenient to consider the definition from a coordinate-space correlator,

$$
N_{P, W}(\vec{k})=\int \frac{d^{3} \xi}{(2 \pi)^{3}} e^{-i \vec{k} \cdot \vec{\xi}}\left\langle P\left|\bar{\psi}(0) \gamma^{0} W(0, \vec{\xi}) \psi(\vec{\xi})\right| P\right\rangle,
$$

where the Dirac matrix $\gamma^{0}$ ensures that it is a number density. Clearly, it is a static quantity without timedependence and can be calculated in Euclidean field theories, in contrast to Eq. (8) for partons. The gauge invariance is ensured by the Wilson line $W(0, \vec{\xi})$ between the quark fields separated by $\vec{\xi}$, which is defined in the fundamental representation of the color $\mathrm{SU}(3)$ group. There are infinitely many choices for the Wilson line, generating infinitely many momentum densities. For example, one can choose a straight-line link between 0 and $\vec{\xi}$. One can also let the Wilson line run from the fields along the $z$-direction for a long distance (if not infinity) before joining them together along the transverse direction (a staple).

For its obvious connection to the PDFs, we consider a transverse-momentum integrated, longitudinalmomentum distribution,

$$
\begin{aligned}
N_{P}\left(k^{z}\right) & =\int d^{2} \vec{k}_{\perp} N_{P, W}(\vec{k}) \\
& =\int \frac{d z}{2 \pi} e^{-i k^{z} z}\left\langle P\left|\bar{\psi}(0) \gamma^{0} W(0, z) \psi(z)\right| P\right\rangle,
\end{aligned}
$$

where we ignore the question of convergence at large $\left|\vec{k}_{\perp}\right|$. Now the gauge-link $W(0, z)$ is naturally taken as a straight-line,

$$
\begin{aligned}
W(0, z) & =\exp \left(-i \int_{0}^{z} A^{z^{\prime}}\left(z^{\prime}\right) d z^{\prime}\right) \\
& =\exp \left(i \int_{\infty}^{0} A^{z^{\prime}}\left(z^{\prime}\right) d z^{\prime}\right) \exp \left(i \int_{z}^{\infty} A^{z^{\prime}}\left(z^{\prime}\right) d z^{\prime}\right) \\
& =W^{\dagger}(\infty, 0) W(\infty, z),
\end{aligned}
$$

where in the second line we have split the gauge link into two, going from $z$ to the infinity and coming back from the infinity to zero. We can define a "gauge-invariant" quark field

$$
\Psi(\vec{\xi})=W(\infty, \vec{\xi}) \psi(\vec{\xi})
$$


and the above density becomes,

$$
N_{P}\left(k^{z}\right)=\int \frac{d z}{2 \pi} e^{-i k^{z} z}\left\langle P\left|\bar{\Psi}(0) \gamma^{0} \Psi(z)\right| P\right\rangle,
$$

where again we have not considered UV divergences. The momentum distribution defined above has been called quasi- $P D F$, but it is really a physical momentum distribution in a proton of momentum $P$.

In the rest frame of the proton, $N_{P=0}\left(k^{z}\right)$ is symmetric in positive and negative $k^{z}$, probably peaks around $k^{z}=0$ and decays away as $k^{z} \rightarrow \pm \infty$. Due to the perturbative QCD effects, it decays algebraically at large $k^{z}$, instead of exponentially. Because of this property, the high moments of the distribution, $\int d k^{z}\left(k^{z}\right)^{n} N_{0}\left(k^{z}\right)$ with $n>0$, have the standard QFT UV divergences.

As $P$ becomes non-zero and large, the peak $N_{P}\left(k_{z}\right)$ will be around $\alpha P^{z}$, where $\alpha$ is a constant of order one. The density at negative $k^{z}$ becomes smaller, but not zero. This is due to the so-called backward-moving particles from the large momentum kick in perturbation theory. For the same reason, the density at $k^{z}>P^{z}$ is not zero either.

$N_{P}\left(k^{z}\right)$ has a renormalization scale dependence because the quark fields must be renormalized. One can choose DR and modified minimal subtraction ( $\overline{\mathrm{MS}})$ scheme. Any other regularization scheme can be converted into this one perturbatively. For $z \neq 0$, the only renormalization necessary is the quark wave function (with anomalous dimension $\gamma_{F}$ ) in the $A^{z}=0$ gauge, because the linear divergence associated with the gauge link vanishes in the $\overline{\mathrm{MS}}$ scheme. More extensive discussions on the renormalization issue, particularly about non-perturbative renormalization, will be made in the following section.

As an example showing how the parton momentum density depends on $P$, we depict in Fig. 4 the quark wave function amplitude of a meson in the 't Hooft model $(1+1$ dimensional QCD with $N_{c} \rightarrow \infty$ ) ('t Hooft, 1974), the square of which yields the quark momentum density. In this model, a meson of momentum $P^{\mu}$ can be built as

$$
\begin{aligned}
\left|P_{n}^{\mu}\right\rangle=\int \frac{d k}{2 \pi|P|} & {\left[M(k-P, k) \phi_{n}^{+}(k, P)\right.} \\
+ & \left.M^{\dagger}(k, k-P) \phi_{n}^{-}(k, P)\right]|0\rangle,
\end{aligned}
$$

where $M(p, k)=\sum_{i} d_{-p}^{i} b_{k}^{i} / \sqrt{N_{c}}$, and $M^{\dagger}(p, k)=$ $\sum_{i} b_{k}^{i \dagger} d_{-p}^{i \dagger} / \sqrt{N_{c}}$ are annihilation and creation operators for quark-antiquark pairs. The corresponding wave function amplitudes, $\phi_{n}^{+}(k, P)$ and $\phi_{n}^{-}(k, P)$, satisfy a pair of equations first derived in (Bars and Green, 1978).

The meson bound state defined above has a welldefined large-momentum limit. The wave functions can be expanded in $1 / P$, with the corrections starting from $(1 / P)^{2}$. The momenta of the constituents, $k$ and $P-k$, scale in this limit. When plotted as a function of $x=$ $k / P$, the change in the wave function with the magnitude of the momentum is shown in Fig. [4.
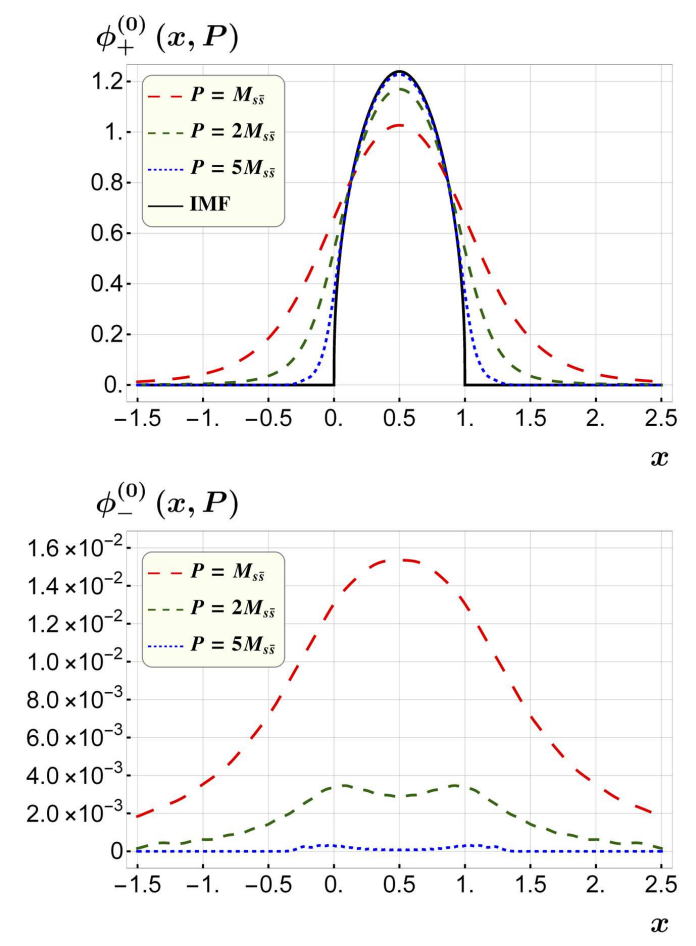

FIG. 4: Wave function amplitudes of a meson in the 't Hooft model at different external momenta (Jia et al., 2017).

\section{B. Momentum Renormalization Group}

In this subsection, we consider how to calculate the external momentum $P$ dependence of physical observables discussed in the previous subsection. Clearly, the dependence is related to the boost properties of the operators under consideration, namely their commutation relations with the boost generators, $\hat{K}^{i}$. We argue that in the large momentum limit, one has a momentum $R G E$ which is a differential equation relating properties of the system at different momenta. Momentum RGE will be, in the end, related to the renormalization properties of the observables on the LF.

Consider a generic operator $\hat{O}$, and its matrix element in a state with momentum $P$,

$$
O(P)=\langle P|\hat{O}| P\rangle .
$$

We calculate the momentum dependence by writing $|P\rangle=\exp (-i \omega(P) \hat{K})|P=0\rangle$, where $\hat{K}$ is the boost operator along the momentum direction and $\omega$ is a boost parameter depending on $P$. Taking a derivative with respect to the boost parameter gives

$$
\frac{d O(P)}{d P}=i \frac{d \omega(P)}{d P}\langle P|[\hat{O}, \hat{K}]| P\rangle .
$$

The r.h.s. of the equation depends on the commutator $[\hat{O}, \hat{K}]$, i.e., the boost properties of the operator. For a scalar operator, the commutation relation vanishes, and $O(P)$ is frame independent. For a vector operator, 


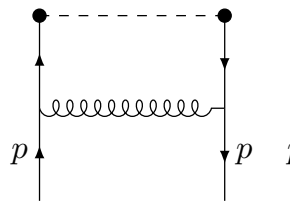

(a)

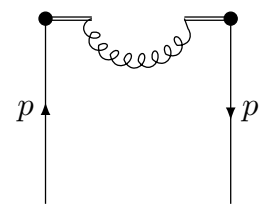

(d)

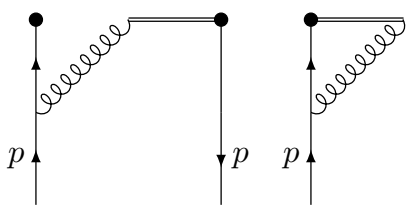

(b)

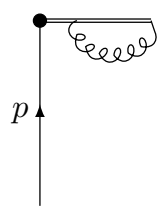

(e) (c)

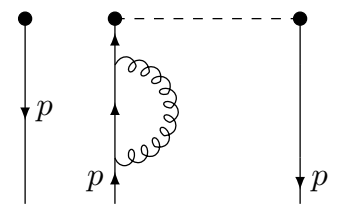

(f)
FIG. 5: One-loop diagrams for the quasi-PDF in a free quark state in the Feynman gauge. The conjugate diagrams of (b), (c), (e), (f) do contribute but are not shown here.

the commutation relation resembles that of an energymomentum four-vector, and the result is the standard Lorentz transformation of a four-vector. For nonlocal operators, the commutation relation requires the elementary formula,

$$
\left[J^{\mu \nu}, \phi_{i}(x)\right]=i\left[l^{\mu \nu} \delta_{i j}+S_{i j}^{\mu \nu}\right] \phi_{j}(x),
$$

where $l^{\mu \nu}=-i\left(x^{\mu} \partial^{\nu}-x^{\nu} \partial^{\mu}\right)$ is the OAM operator and $S^{\mu \nu}$ is the intrinsic spin matrix. Thus one of the fields is now $\phi_{i}(t=\sinh \omega z, 0,0, \cosh \omega z)$ which generates a time-dependent correlation function.

In the large-momentum limit, because of asymptotic freedom, the $P$-dependence is calculable in perturbation theory, and Eq. (27) simplifies. One obtains the momentum or boost RGE (Ji, 2014),

$$
\begin{aligned}
& \frac{d O(P)}{d P}=\lim _{\Delta P \rightarrow 0}[O(P+\Delta P)-O(P)] / \Delta P \\
& \stackrel{P \gg M}{\longrightarrow} C\left(\alpha_{s}(P)\right) \otimes O(P)+\mathcal{O}\left(M^{2} / P^{2}\right) .
\end{aligned}
$$

where $C\left(\alpha_{s}(P)\right)$ is a perturbative expansion in the strong coupling $\alpha_{s}$. The symbol " $\otimes$ " can be a simple multiplication or certain form of convolution, depending on the observable $O(P)$ studied. The proof of the above equation is non-trivial, and it can be analyzed on a case-by-case basis. There can be mixings among a set of independent operators with the same quantum numbers. The momentum RGEs are very similar to those for scale transformation or that for the coarse graining of a Hamiltonian. That the two are connected in some cases may be traced to Lorentz symmetry.

As an example of the momentum RGE, we calculate the quark momentum distribution in a perturbative quark state using Eq. (24). Since it is gauge invariant, we can calculate it in any gauge, for example, the Feynman gauge. The one-loop diagrams in QCD are shown in Fig. 5. There are two sources of UV divergences, one is the logarithmic divergences from the vertex and selfenergy diagrams, and the other is the linear divergence in the self-energy of the Wilson line. For the moment, we will use transverse momentum cut-off, $\Lambda_{\mathrm{UV}}$, as the UV regulator. Using $y=k^{z} / P^{z}$, the one-loop result reads for a large momentum quark (Xiong et al., 2014),

$$
\begin{aligned}
& \tilde{q}^{(1)}\left(y, P^{z}, \Lambda_{\mathrm{UV}}\right)=\frac{\alpha_{s} C_{F}}{2 \pi} \\
& \times\left\{\begin{array}{cl}
\frac{1+y^{2}}{1-y} \ln \frac{y}{y-1}+1+\frac{\Lambda_{\mathrm{UV}}}{(1-y)^{2} P^{z}}, & y>1 \\
\frac{1+y^{2}}{1-y} \ln \frac{\left(P^{z}\right)^{2}}{m^{2}}+\frac{1+y^{2}}{1-y} \ln \frac{4 y}{1-y} & 0<y<1 \\
-\frac{4 y}{1-y}+1+\frac{\Lambda_{\mathrm{UV}}}{(1-y)^{2} P^{z}}, & 0<1 \\
\frac{1+y^{2}}{1-y} \ln \frac{y-1}{y}-1+\frac{\Lambda_{\mathrm{UV}}}{(1-y)^{2} P^{z}}, & y<0
\end{array}\right.
\end{aligned}
$$

where we have ignored all power-suppressed contributions and keep the leading $P^{z}$ dependence only. There is an additional contribution of the form $\delta Z_{1}\left(\Lambda_{\mathrm{UV}} / P^{z}\right) \delta(y-1)$.

The above result has several interesting features:

- The distribution does not vanish outside $[0,1]$. The radiative gluon can carry a large negative momentum fraction, resulting in a recoiling quark carrying larger momentum than the parent quark, and thus $y>1$. The same gluon can also carry a momentum larger than $P^{z}$, making the active quark have $y<0$.

- While the above effect is easy to understand perturbatively, it is surprising that a scaling contribution remains outside $[0,1]$ in the IMF. As the proton travels faster, one might think any constituent has a momentum $k^{z}$ positive from Lorentz transformation. However, the order of limits matters because no matter how large the parent-quark momentum is, there are always quarks with much larger momentum, i.e., $k^{z} \gg P^{z} \gg \Lambda_{\mathrm{QCD}}$. In this sense, Feynman's parton model does not describe the exact properties of the momentum distribution in a large-momentum nucleon.

- The contribution outside $[0,1]$ at one-loop is entirely perturbative because of the absence of any infrared (IR) divergence. This is no longer true at two-loop level, but the contribution depends only on the same one-loop IR physics in $[0,1]$.

- The distribution for $y$ in $[0,1]$ has a term depending on $\ln P^{z}$. This dependence reflects that the quark substructure is resolved as a function of $P^{z}$, an interesting feature of boost. This dependence is perturbative in the sense that the derivative is IR safe,

$$
\begin{aligned}
& P^{z} \frac{d \tilde{q}\left(y, P^{z}, \Lambda_{\mathrm{UV}}\right)}{d P^{z}} \\
& =\frac{\alpha_{s} C_{F}}{\pi}\left[\left(\frac{1+y^{2}}{1-y}\right)_{+}-\frac{3}{2} \delta(1-y)\right] .
\end{aligned}
$$


Apart from the $\delta$-function term, the r.h.s. is similar to the one-loop quark splitting function in DGLAP evolution Altarelli and Parisi, 1977; Dokshitzer, 1977; Gribov and Lipatov, 1972). Therefore one might suspect that the momentum dependence is closely related to the familiar renormalization scale evolution in the PDFs. In fact, the physics is just the other way around: It is the hadron-momentum dependence of the physical momentum distribution that generates the DGLAP evolution in the infinitemomentum limit. One can derive an all-order momentum RGE for the momentum distribution function. Momentum RGE also provides a method to sum over the large logarithms of the momentum.

- There is a singularity at $y=1$. This singularity is generated from soft-gluon radiation. Fortunately, this singularity combined with the virtual contribution yields a finite result.

- There is a linear divergence in the cut-off regulator, leading to $\Lambda_{\mathrm{UV}} / P^{z}$ term, which is absent in DR. Thus, to keep $1 /\left(P^{z}\right)^{2}$ power counting, it is important to work in a renormalization scheme where this term does not exist.

We can also move on to study the hadron momentum RGEs of other structural properties considered in the previous subsection. In particular, the RGE for TMD distributions will lead to the familiar rapidity RGE in the literature. We reserve these discussions to Sec. V.

\section{Effective Field Theory Matching to PDFs}

As we have seen in the previous subsection, the momentum distributions of the constituents (now called quasi-PDFs in the literature) in a proton at large $P$ are different from the PDFs or LF distributions in many ways. In particular, the momentum fraction $y$ in a physical momentum distribution is not limited to $[0,1]$ due to backward moving particles, which is the case even in the $P \rightarrow \infty$ limit. In fact, the infinite-momentum limit is not analytical due to the presence of $\ln P$.

However, partons are effective objects arising from a different limit $\Lambda_{\mathrm{UV}} \ll P \rightarrow \infty$. There is also an important computational advantage in taking the naive limit $P \gg \Lambda_{\mathrm{UV}}$ in perturbative calculations: Feynman integrals have one fewer four-momentum. Therefore, this limit of QFTs serves as a reference system where the structure of the bound states is manifestly independent of the hadron momentum, and is similar to scale-invariant critical points at which second-order phase transitions occur in condensed matter systems. However, the theory in the naive IMF limit introduces additional UV divergences.

Therefore, the partons in QCD are very similar to the infinitely heavy quarks in HQET (Manohar and Wise,
2000). In certain QCD systems, heavy quarks such as the bottom quark are present, and their masses are much larger than the typical QCD scale $\Lambda_{\mathrm{QCD}}$. In this case, one might study the dependence on the heavy quark mass by expanding around $m_{Q}=\infty$. This expansion will generally produce a power series in $1 / m_{Q}$. However, the limits of taking $\Lambda_{\mathrm{UV}} \rightarrow \infty$ and infinite heavy-quark mass limits are not interchangeable, due to the presence of the large logarithms $\ln m_{Q}$. In an EFT approach, one takes the $m_{Q} \rightarrow \infty$ limit first, this will result in a new theory with different UV behavior, but without the heavy-quark mass, and symmetries among very different heavy-quark systems become manifest. The renormalization of the extra UV divergences yields a RGE which can be used to resum large quark-mass logarithms.

Therefore, the momentum distribution at large- $P$ differs from the parton distributions only in the order of limits, their IR non-perturbative physics is the same. In asymptotically free theories such as QCD, differences (or discontinuities) in taking the limits of $P \gg \Lambda_{\mathrm{UV}}$ and $\Lambda_{\mathrm{UV}} \gg P \rightarrow \infty$ are perturbatively calculable, as only the high-momentum modes matter. The differences are called matching coefficients. Therefore, one is able to write down a power expansion for the momentum-dependent distribution (quasi-PDF) in terms of the PDF (Izubuchi et al., 2018; Ma and Qiu, 2018a, b; Xiong et al., 2014),

$$
\begin{aligned}
\tilde{q}\left(y, P^{z}, \mu\right)=\int_{-1}^{1} \frac{d x}{|x|} & C\left(\frac{y}{x}, \frac{\mu}{x P^{z}}\right) q(x, \mu) \\
& +\mathcal{O}\left(\frac{\Lambda_{\mathrm{QCD}}^{2}}{\left(y P^{z}\right)^{2}}, \frac{\Lambda_{\mathrm{QCD}}^{2}}{\left((1-y) P^{z}\right)^{2}}\right)
\end{aligned}
$$

where the power correction is suppressed by the parton momentum $y P^{z}$ and the spectator momentum $(1-$ y) $P^{z}$ (Ji et al., 2020b). This expansion may be also called a factorization formula, as the quasi-PDF contains all the IR physics in the PDF, and $C$ involves only UV physics. As we will discuss extensively in the next section, this factorization formula is true to all orders in perturbation theory. The above relation allows us to calculate the LF parton physics from the momentum distribution at large $P$. Since the expansion parameter is $\Lambda_{\mathrm{QCD}}^{2} /\left(y P^{z}\right)^{2}$ and $\Lambda_{\mathrm{QCD}}^{2} /\left((1-y) P^{z}\right)^{2}$, for intermediate $y$ one might not need very large $P^{z}$ to neglect the power corrections.

The above relation between the two quantities has a simple explanation in terms of the Lorentz boost: Consider the spatial correlation along $z$ shown in Fig. [6 in a large momentum state. It can be seen as approaching the LF one in the rest frame of the proton. In other words, we are using a near-LF correlation to approximate a LF correlation. Accordingly, we can invert the above equation recursively to express the PDF in terms of quasiPDF with their differences being taken care of through 
the perturbative matching $\tilde{C}$ and power corrections,

$$
\begin{aligned}
q(x, \mu)=\int_{-\infty}^{\infty} \frac{d y}{|y|} \tilde{C}\left(\frac{x}{y}, \frac{\mu}{y P^{z}}\right) \tilde{q}\left(y, P^{z}, \mu\right) \\
+\mathcal{O}\left(\frac{\Lambda_{\mathrm{QCD}}^{2}}{\left(x P^{z}\right)^{2}}, \frac{\Lambda_{\mathrm{QCD}}^{2}}{\left((1-x) P^{z}\right)^{2}}\right) .
\end{aligned}
$$

The above equation has an EFT interpretation: The parton physics is calculated in an effective field theory with physical momentum scale from 0 to $P$, whereas the physics from degrees of freedom from $P$ to $\infty$ can be integrated out to generate the perturbative coefficients $\tilde{C}$ and the high-order terms in $1 /\left(P^{z}\right)^{2}$. In constrast to HQET, the full QCD degrees of freedom are used in LaMET calculations. In other words, the effective Lagrangian of LaMET is the standard QCD one, while the large momentum $P$ for expansions appears only in the external states.

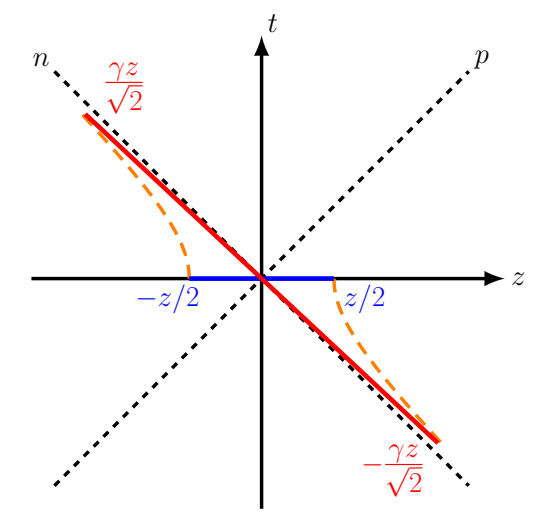

FIG. 6: The line segment in the $z$-direction in the frame of a large-momentum hadron. Through Lorentz boost, it is equivalent to a line segment of length $\sim \gamma z$ close to the light-one in the hadron state of zero momentum. Here $\gamma z / \sqrt{2}$ is the length of projection of the boosted line segment to the light-cone direction $n$. Thus, we call the dimensionless variable $\lambda=z P^{z} \sim \gamma z M$ as the quasi light-cone distance.

\section{Recipe for Parton Physics in LaMET}

The principle of LaMET is to simulate the timedependence of parton observables through external states at large momentum. Thus, we can generalize the discussions in the previous subsection to any type of physical observables for the large momentum proton, which will be generally called quasi-parton observables. Examples will be given in the later sections including transversemomentum dependent distributions and LF wave functions.

Consider any Euclidean quasi-observable $O$ which depends on a large hadron momentum $P^{z}$ and UV cut-off $\Lambda_{\mathrm{UV}} \gg P^{z}$. Using asymptotic freedom, we can system- atically expand the $P^{z}$ dependence,

$$
O\left(P^{z}, \Lambda_{\mathrm{UV}}\right)=Z\left(\frac{P^{z}}{\Lambda_{\mathrm{UV}}}, \frac{P^{z}}{\mu}\right) \otimes o(\mu)+\mathcal{O}\left(\frac{\Lambda_{\mathrm{QCD}}^{2}}{\left(P^{z}\right)^{2}}\right)+\ldots,
$$

where $\otimes$ refers to a convolution if appropriate, and $Z$ factorizes all the perturbative dependence on $P^{z}$ and does not contain any IR divergence. The quantity $o(\mu)$ is defined in a theory with $P^{z} \rightarrow \infty$, exactly as in Feynman's parton model. In fact, $o(\mu)$ is a LF correlation containing all the IR collinear (and soft) singularities. The important point of the expansion is that it may converge at moderately large $P^{z}$, say a few $\mathrm{GeV}$, allowing access to quantities needed for very large $P^{z}$ (a few TeV). One can also use the large boost-factor $\gamma=P^{z} / M$ as the expansion parameter $1 / \gamma$.

Momentum dependence of the quasi-observables can be studied through momentum RGEs. Defining the anomalous dimension through

$$
\gamma_{P}\left(\alpha_{s}\right)=\frac{1}{Z} \frac{\partial Z}{\partial \ln P^{z}}
$$

it follows that

$$
\frac{\partial O\left(P^{z}\right)}{\partial \ln P^{z}}=\gamma_{P}\left(\alpha_{s}\right) \otimes O\left(P^{z}\right),
$$

up to power corrections. One can resum large logarithms involving $P^{z}$ using the above equation.

When taking $P^{z} \rightarrow \infty$ first in $O\left(P^{z}\right)$ before a UV regularization is imposed, one recovers from $\hat{O}$ the lightcone operator $\hat{o}$, by construction. On the other hand, the physical matrix element is calculated at a large $P^{z}$, with UV regularization such as the lattice cut-off imposed first. Thus the difference between the matrix elements of $\hat{o}$ and $\hat{O}$ is a matter of the order of limits. This is the standard set-up for an EFT. The different limits do not change the IR physics. In fact, the factorization in terms of Feynman diagrams can be proved order by order as in the renormalization program, as discussed in the following section.

The parton physics can be calculated more directly by reversing Eq. (35) to produce an EFT expansion,

$$
o(\mu)=\tilde{Z}\left(\frac{P^{z}}{\Lambda_{\mathrm{UV}}}, \frac{P^{z}}{\mu}\right) \otimes O\left(P^{z}, \Lambda_{\mathrm{UV}}\right)+\mathcal{O}\left(\frac{\Lambda_{\mathrm{QCD}}^{2}}{\left(P^{z}\right)^{2}}\right)+\ldots .
$$

Thus, to compute any parton observable defined by an operator made of LF dynamical fields, $\hat{o}$, one constructs a time-independent version $\hat{O}$ which, under an infinite Lorentz boost, approaches $\hat{o}$. Then, one calculates the matrix element of $\hat{O}$ in a hadron with large momentum $P^{z}$ using whatever approach (lattice QCD is an obvious choice for the time-independent operator $\hat{O}$ ) and then uses Eq. (38) to systematically approximate the parton 
observable. Usually the matrix element of $\hat{O}$ depends on $P^{z}$ as well as all the lattice UV artifacts. In principle, the latter does not affect the EFT expansion and will be cancelled by the matching coefficient $\tilde{Z}$ and higher order terms in the expansion. However, in practical applications such as the quasi-PDF calculations, a nonperturbative renormalization is still necessary to remove all the power divergences to ensure a continuum limit.

\section{E. Universality}

LaMET provides a framework to systematically compute partonic observables on the LF from the properties of a large-momentum proton. However, the relationship is not one-to-one. There can be infinitely many possible Euclidean operators in the large-momentum proton which generate the same LF observable. This is because the large-momentum physical states have built-in collinear (as well as soft) parton modes, and upon acting on a Euclidean operator, they help to project out the leading LF physics. All operators projecting out the same LF physics form a universality class. Accordingly, in the operator formulation for parton physics such as SCET, one uses LF operators to project out parton physics off the external states of any momentum, including $P=0$.

The concepts such as universality class have been used in critical phenomena in condensed matter physics, where systems with different microscopic Hamiltonians can have the same scaling properties near their critical points. Critical phenomena correspond to the IR fixed points of the scale transformation, and are dominated by physics at long-distance scales. In the present case, parton physics arises from the infinite-momentum limit, $P=\infty$, which is a UV fixed point of the momentum RGEs. It is the longitudinal short distance (or large momentum) physics that is relevant at the fixed point. However, the short distance here does not mean everything is perturbative. The part that is non-perturbative characterizes the partonic structure of the proton. The critical region near $P=\infty$ acts as a filter to select only the physics that is relevant, so universality classes emerge.

In the case of unpolarized PDFs, the initial proposal in LaMET starts from the matrix element of the following operator ( Ji, 2013),

$$
O_{1}(z)=\bar{\psi}(0) \gamma^{z} W(0, z) \psi(z) .
$$

However, from Constantinou and Panagopoulos, 2017; Radyushkin, 2017b),

$$
\mathrm{O}_{2}(z)=\bar{\psi}(0) \gamma^{0} W(0, z) \psi(z),
$$

and the leading contributions in the large-momentum expansion are the same. One can also consider any linear combination of the two. In (Jia et al., 2018), the calculations have been done with these two operators in the 't Hooft model, and the results have been compared at different hadron momenta. For lattice simulations, an important issue is about the operator mixing, which depends on specific choices of the operators in the universality class.

Another example of Euclidean operators for PDFs is the current-current correlators in a pure space separation,

$$
O_{3}(z)=J^{\mu}(0) J^{\nu}(z),
$$

where $J^{\mu}$ is, for example, an electromagnetic current. This type of correlator was first considered in (Bali et al., 2018b; Braun and Müller, 2008) for calculating pion DA, and recently has been suggested to calculate PDFs with generalized bilocal "currents" Ma and Qiu, 2018a). When the matrix elements are calculated in the large-momentum states, $O(z)$ falls into the same universality class as the operators in Eqs. (39) and (40). Instead of using light quarks as the intermediate propagator in $O(z)$, one can have a number of other choices for LaMET applications, including scalars (Abada et al., 2001; Aglietti et al., 1998) and heavy-quarks (Detmold and Lin, 2006). One can also similarly work with quark bilinear operators in any physical gauge which become the light-front one in the large momentum limit (Gupta et al., 1993).

Another important example of universality class is the gluon helicity contribution to the spin of the proton, as we will discuss in detail in Sec. IV.D. The gluon spin operator $\vec{E} \times \vec{A}$ is gauge-dependent. However, in physical gauges where the transverse degrees of freedom are dynamical, its matrix element is the same in the largemomentum limit. Therefore, one can potentially choose different gauges to perform calculations at finite momentum on lattice, such as Coulomb gauge $\vec{\nabla} \cdot \vec{A}=0$, axial gauge $A^{z}=0$ or temporal gauge $A^{0}=0$. Different gauge choices will have different UV properties $(\ln P)$ and hence different matching conditions. However, the IR part of the matrix element is the same (Hatta et al., 2014).

At a practical level, it is very useful to find which operator has the fastest convergence in the LaMET expansion. The current-current correlators use the light-quark propagator to simulate the light-like Wilson line (sometimes called light-ray). The quasi-PDF approach not only starts from a quantity with clear physical meaning (a momentum distribution), but also generates the needed Wilson line simply by rotating a space-like one, shown in Fig. 6. Thus, it is plausible that the quasi-PDF will provide mathematically the fastest large- $P$ convergence than the other choices.

\section{RENORMALIZATION AND MATCHING FOR PDFS}

In this section, we consider the LaMET application to calculating the simplest collinear PDFs, which have been most extensively studied in the literature so far. 
Although universality allows one to extract the collinear PDFs from the matrix elements of a wide class of operators evaluated at large momentum, we will focus on physical observables closely resembling the collinear PDFs, i.e., the quark and gluon momentum distributions or the quasi-PDFs. We also discuss the coordinate-space factorization approach in which the pseudo-PDF and currentcurrent correlators have been studied.

We mainly review the technical progress made in renormalization and matching using the quasi-PDFs. The matching can be done in principle at the bare matrix elements level, since the factorization formula like Eq. (33) is valid for both bare and renormalized momentum distributions. All the UV divergences in the bare quasiPDF can be factorized into the matching coefficient $C$, and the latter automatically renormalizes the bare lattice matrix elements, so the continuum limit can be taken afterwards. However, such a matching coefficient then has to be calculated in lattice perturbation theory, which is computationally challenging and converges slowly (Lepage and Mackenzie, 1993). More importantly, the quasi-PDF contains linear power divergence under UV cutoff regularization due to the Wilson line selfenergy (Ji, 2013; Xiong et al., 2014), which makes it impossible to take the continuum limit with fixed-order calculations in lattice perturbation theory. Though the latter problem can be improved by resumming the linear and possibly logarithmic divergences, it is usually preferred to nonperturbatively renormalize the quasi-PDFs on the lattice, after which a continuum limit can be taken and a perturbative matching can be done in the continuum theory. To this end, a thorough understanding of the renormalizability of Wilson-line operators defining the quasi-PDFs is required. In addition to renormalization, the applications of LaMET rely on the validity of the large-momentum factorization formula Eq. (33), which can be proven in perturbation theory to all orders by showing that the collinear divergences are the same in the momentum distributions and light-cone PDFs.

We begin in Sec. III.A with the proof of multiplicative renormalizability of the Wilson-line operators that define the quasi-PDFs. We first work in the continuum theory with $\overline{\mathrm{MS}}$ scheme, and then generalize the conclusion to lattice theory. Next, in Sec. III.B we outline the factorization theorem for momentum distributions to all orders in perturbation theory, and state the form of convolution between the matching coefficient and the PDF. In Sec. III.C we show that the factorization theorem has an equivalent form in coordinate space, which can be used as an alternate route to extract PDFs from lattice matrix elements. Finally, we discuss the nonperturbative renormalization of quasi-PDFs on the lattice and their matching to the $\overline{\mathrm{MS}} \mathrm{PDF}$ in Sec. III.D

\section{A. Renormalization of Nonlocal Wilson-Line Operators}

The momentum distributions of the proton are defined from equal-time nonlocal Wilson line operators of the form in Eq. (21). In this subsection, we review the renormalization of these spacelike nonlocal operators (the renormalization of lightlike nonlocal operators defining the PDFs can be found in (Collins, 2011a; Collins and Soper, 1982b)). We first discuss their renormalization in DR using an auxiliary field approach, followed by the discussion on similar gluon operators. We then consider power divergences in the momentum cutoff type of UV regularization. The conclusion is that they are all multiplicatively renormalizable with a finite number of mixings with other operators.

\section{Renormalization of nonlocal quark operators}

We are interested in operators of the following kind,

$$
O_{\Gamma}(z)=\bar{\psi}\left(\frac{z}{2}\right) \Gamma W\left(\frac{z}{2},-\frac{z}{2}\right) \psi\left(-\frac{z}{2}\right) .
$$

Since the Wilson line $W\left(z_{1}, z_{2}\right)$ is a path-ordered integral of gauge fields, it is not obvious that such operators are multiplicatively renormalizable. The renormalization of non-lightlike Wilson loops and Wilson lines has been studied in early literature (Craigie and Dorn, 1981; Dotsenko and Vergeles, 1980), and the all-order proof of their multiplicative renormalizability was first made using diagrammatic methods in Craigie and Dorn, 1981; Dotsenko and Vergeles, 1980) and then the functional formalism of gauge theories in (Dorn, 1986). The same conclusion was conjectured to hold also for the quark bilinear operator $O_{\Gamma}(z)$, whose renormalization takes the following form (Chen et al., 2017; Ishikawa et al., 2016; Musch et al., 2011),

$$
O_{\Gamma}^{B}(z, \Lambda)=Z_{\psi, z}(\Lambda, \mu) e^{\delta m(\Lambda)|z|} O_{\Gamma}^{R}(z, \mu),
$$

where " $B$ " and " $R$ " stand for bare and renormalized operators respectively, and all the fields and couplings in $O_{\Gamma}^{B}(z, \Lambda)$ are bare ones which depend on the UV cutoff $\Lambda$. $\delta m(\Lambda)$ is the "mass correction" of the Wilson line, which includes all the linear power divergences of its self-energy. $Z_{\psi, z}(\Lambda, \mu)$ includes all the logarithmic divergences from wavefunction and vertex renormalizations.

An early two-loop study of the quasi-PDF in the $\overline{\mathrm{MS}}$ scheme indeed indicated the multiplicative renormalizability of $O_{\Gamma}(z)$ (Ji and Zhang, 2015). The first rigorous proof of Eq. (43) was given in the auxiliary "heavy quark" field formalism (Green et al., 2018; Ji et al., 2018) which was used to prove the renormalizability of Wilson lines (Arefeva, 1980; Dorn, 1986; Gervais and Neveu, 1980; Samuel, 1979). This formalism is defined by extending the QCD Lagrangian to include the auxiliary 
"heavy quark" fields $Q, \bar{Q}$ and their gauge interaction,

$$
\mathcal{L}=\mathcal{L}_{\mathrm{QCD}}+\bar{Q}_{0} i n_{z} \cdot D_{0} Q_{0},
$$

where the subscript " 0 " denotes bare quantities. $n_{z}^{\mu}=$ $(0,0,0,1)$ is the direction vector of the spacelike Wilson line $W(z, 0), D_{0}^{\mu}=\partial^{\mu}+i g_{0} A_{0}^{\mu}$, and $Q_{0}$ is a color-triplet scalar Grassmann field in the fundamental representation of SU(3). Note that if we replace $n_{z}^{\mu}$ with the timelike vector $n_{t}^{\mu}=(1,0,0,0)$, then Eq. (44) yields the leading order HQET Lagrangian.

In the theory defined by Eq. (44), the Wilson line can be expressed as the connected two-point function of the "heavy-quark" fields,

$$
\left\langle Q_{0}(\xi) \bar{Q}_{0}(\eta)\right\rangle_{Q}=S_{0}^{Q}(\xi, \eta),
$$

where $\xi$ and $\eta$ are space-time coordinates, and $\langle\ldots\rangle_{Q}$ stands for integrating out the auxiliary fields. The above equation is valid up to the determinant of $i n_{z} \cdot D_{0}$, which is a constant and can be absorbed into the normalization of the generating functional (Mannel et al., 1992). The Green's function $S_{0}^{Q}(\xi, \eta)$ satisfies

$$
i n_{z} \cdot D_{0}(\xi) S_{0}^{Q}(\xi, \eta)=\delta^{(4)}(\xi-\eta),
$$

which has the solution

$$
S_{0}^{Q}(\xi, \eta)=W\left(\xi^{3}, \eta^{3}\right) \theta\left(\xi^{3}-\eta^{3}\right) \delta\left(\xi^{0}-\eta^{0}\right) \delta^{(2)}\left(\vec{\xi}_{\perp}-\vec{\eta}_{\perp}\right)
$$

with a proper choice of boundary condition. In this way, the Wilson-line operator $O_{\Gamma}^{B}(z)$ can be replaced by the product of two local composite operators averaged over all the "heavy-quark" field configurations (Dorn, 1986),

$$
\begin{aligned}
O_{\Gamma}^{B}(z)= & \int d^{4} \xi \delta\left(\xi^{3}-z\right) \\
& \times\left\langle\bar{\psi}_{0}\left(\frac{\xi}{2}\right) Q_{0}\left(\frac{\xi}{2}\right) \Gamma \bar{Q}_{0}\left(-\frac{\xi}{2}\right) \psi_{0}\left(-\frac{\xi}{2}\right)\right\rangle_{Q},
\end{aligned}
$$

where the UV regulator is suppressed.

Consequently, the renormalization of $O_{\Gamma}^{B}(z)$ is reduced to that of the two local "heavy-to-light" currents

$$
J^{B}=\bar{Q}_{0} \psi_{0} .
$$

The renormalizability of this auxiliary field theory has been proven using the standard functional techniques for gauge theories (Dorn, 1986). After fixing the covariant gauge and introducing the ghost fields, the theory including the auxiliary "heavy-quark" has a residual BRST symmetry, from which one can derive the Ward-Takahashi identities to show that all the UV divergences of the Green's functions can be subtracted with a finite number of local counterterms. In analogy, the same method has also been used to prove the all-order renormalization of HQET in perturbation theory (Bagan and Gosdzinsky, 1994).
According to (Dorn, 1986), the "heavy-quark" Lagrangian can be renormalized in a covariant gauge as

$$
\begin{aligned}
\mathcal{L} & =\mathcal{L}_{\mathrm{QCD}}\left[g_{0}, \psi_{0}, A_{0}, c_{0}\right]+\bar{Q}_{0} i n_{z} \cdot D_{0} Q_{0} \\
& =\mathcal{L}_{\mathrm{QCD}}[g, \psi, A, c]+\mathcal{L}_{\text {c.t. }}[g, \psi, A, c] \\
& +Z_{Q} \bar{Q}\left(i n_{z} \cdot \partial-i \delta m\right) Q-g Z_{1}^{Q Q g} \bar{Q} n_{z} \cdot A_{a} t^{a} Q
\end{aligned}
$$

where $\mathcal{L}_{\text {c.t. }}[g, \psi, A, c]$ are the $\mathrm{QCD}$ counterterms, and the bare fields and coupling are related to the renormalized ones through

$$
\psi_{0}=Z_{\psi}^{\frac{1}{2}} \psi, A_{0}=Z_{A}^{\frac{1}{2}} A, Q_{0}=Z_{Q}^{\frac{1}{2}} Q, g_{0}=Z_{g} g .
$$

The heavy-quark-gluon vertex renormalization constant $Z_{1}^{Q Q g}$ is related to $Z_{g}$ through the Slavnov-Taylor identities of the auxiliary field theory (Dorn, 1986),

$$
Z_{g}=Z_{1}^{Q Q g} Z_{A}^{-\frac{1}{2}} Z_{Q}^{-1} .
$$

The $i \delta m$ can be regarded as the mass correction of the "heavy quark" except that it is imaginary. For Dirac fermions, the mass correction is logarithmically divergent and proportional to the bare mass, as a result of chiral symmetry; for HQET, the mass correction of the heavy quark is proportional to the UV cutoff, i.e. linearly divergent, which is also expected for the auxiliary field here. Since the proof of renormalizability for this auxiliary field theory is carried out in the $\overline{\mathrm{MS}}$ scheme with DR $(d=4-2 \epsilon)$, all power divergences vanish, so does $\delta m$. Nevertheless, $\delta m$ may include $\mathcal{O}\left(\Lambda_{\mathrm{QCD}}\right)$ contributions due to the renormalon ambiguities which are known to exist in HQET Beneke and Braun, 1994; Bigi et al., 1994).

Since the auxiliary field theory is renormalizable, the renormalization of the operator product in Eq. (48) amounts to the renormalizations of the two "heavy-tolight" currents. Using the standard techniques in quantum field theory (Collins, 1986), one can show recursively that the overall UV divergence of the insertion of $J^{B}$ into Green's functions is absorbed into a renormalization factor $Z_{J}$ to all orders in perturbation theory,

$$
J^{B}=Z_{J} J^{R}=Z_{\psi}^{1 / 2} Z_{Q}^{1 / 2} Z_{V} J^{R},
$$

where $Z_{V}$ is the vertex renormalization constant of the "heavy-to-light" current. The renormalization of heavy-to-light currents in HQET has been calculated up to three-loop order in perturbative QCD Broadhurst and Grozin, 1991; Chetyrkin and Grozin, 2003; Ji and Musolf, 1991; Politzer and Wise, 1988; Shifman and Voloshin, 1987). More recently, it has been argued that the anomalous dimension of the "heavy-to-light" current is identical to that in HQET to all orders (Braun et al., 2020), which is also the case for the "heavy-to-gluon" current that will be discussed below, so the renormalization factors for 
the spacelike and timelike Wilson line operators should be exactly the same.

Using the above results, we can show that

$$
\begin{aligned}
O_{\Gamma}^{B}(z) & =Z_{J}^{2} \int d^{4} \xi \delta\left(\xi^{3}-z\right)\left\langle\bar{J}^{R}\left(\frac{\xi}{2}\right) \Gamma J^{R}\left(-\frac{\xi}{2}\right)\right\rangle_{Q} \\
& =Z_{J}^{2} e^{\delta m|z|} O_{\Gamma}^{R}(z)
\end{aligned}
$$

where $\delta m$ arises from the determinant of $\left(i n_{z} \cdot \partial-\delta m\right)$ in Eq. (50). In this way, we identify that $Z_{\psi, z}=Z_{J}^{2}$ in Eq. (43) which is independent of $\Gamma$. At one-loop order (Stefanis, 1984),

$$
Z_{\psi, z}=1+\frac{\alpha_{s} C_{F}}{4 \pi} \frac{3}{\epsilon_{\mathrm{UV}}},
$$

where the UV regulator $\epsilon_{\mathrm{UV}}$ is to be distinguished from the IR regulator $\epsilon_{\mathrm{IR}}$ in DR.

The multiplicative renormalizability of $O_{\Gamma}^{B}(z)$ has also been proven with a recursive analysis of all-order Feynman diagrams (Ishikawa et al., 2017). In addition to Eq. (43), it was found that $O_{\Gamma}^{B}(z)$ does not mix with gluons or quarks of other flavors. This can also be easily understood within the auxiliary field formalism, as the flavor-changing "heavy-to-light" current does not mix with other operators (Green et al., 2020).

Finally, under lattice regularization we can still use the above techniques to prove Eq. (54), where the mass correction $\delta m$ is now nonvanishing and equal to the lattice UV cutoff $1 / a$ multiplied by a perturbative series in the coupling constant $\alpha_{s}$.

\section{Renormalization of nonlocal gluon operators}

Using the same "heavy-quark" auxiliary field formalism, it has also been proven that the Wilson-line operators for the gluon quasi-PDF are multiplicatively renormalizable (Zhang et al., 2019b), which is echoed by the diagrammatical proof in (Li et al., 2019).

According to LaMET, the gluon quasi-PDF can be defined as ( Ji, 2013)

$$
\tilde{g}\left(x, P^{z}\right)=N \int \frac{d \lambda}{4 \pi x\left(P^{z}\right)^{2}} e^{i \lambda x}\left\langle P\left|O_{g}(z)\right| P\right\rangle,
$$

where $N$ is a normalization factor, and

$$
O_{g}^{B}(z)=g_{\perp, \mu \nu} F_{0, a}^{n_{1} \mu}\left(\frac{z}{2}\right) W^{a b}\left(\frac{z}{2},-\frac{z}{2}\right) F_{0, b}^{n_{2} \nu}\left(-\frac{z}{2}\right)
$$

with $F_{0, a}^{n \mu}=n_{\rho} F_{0, a}^{\rho \mu}$ and $n_{1}^{\mu}, n_{2}^{\mu}$ being either $n_{z}^{\mu}$ or $n_{t}^{\mu}$. $a, b$ are color indices in the adjoint representation. The transverse metric tensor

$$
g_{\perp}^{\mu \nu}=g^{\mu \nu}-n_{t}^{\mu} n_{t}^{\nu} / n_{t}^{2}-n_{z}^{\mu} n_{z}^{\nu} / n_{z}^{2}
$$

and $N=\left(n_{z} \cdot P / n_{t} \cdot P\right)^{\left(n_{1}+n_{2}\right) \cdot n_{t}}$. For lattice implementation, $O_{g}^{B}(z)$ can also be defined as (Dorn, 1986;
Zhang et al., 2019b)

$$
O_{g}^{B}(z)=2 g_{\perp}^{\mu \nu} \operatorname{tr}\left[F_{0, \mu}^{n_{1}}\left(\frac{z}{2}\right) W\left(\frac{z}{2},-\frac{z}{2}\right) F_{0, \nu}^{n_{2}}\left(-\frac{z}{2}\right) W\left(-\frac{z}{2}, \frac{z}{2}\right)\right],
$$

where $F^{\mu \nu}=F_{a}^{\mu \nu} t^{a}$ and $W$ are in the fundamental representation. Similar to Eq. (48), we can express $O_{g}^{B}(z)$ as a product of two local composite operators,

$$
\begin{aligned}
\tilde{O}_{g}^{B}(z) & =\int d^{4} \xi \delta\left(\xi^{3}-z\right) \\
& \times g_{\perp, \mu \nu}\left\langle F_{0, a}^{n_{1} \mu}\left(\frac{\xi}{2}\right) Q_{0}^{a}\left(\frac{\xi}{2}\right) \bar{Q}_{0}^{b}\left(-\frac{\xi}{2}\right) F_{0, b}^{n_{2} \nu}\left(-\frac{\xi}{2}\right)\right\rangle_{Q} \\
& \equiv \int d^{4} \xi \delta\left(\xi^{3}-z\right) g_{\perp}^{\mu \nu}\left\langle J_{n_{1} \mu}^{B}\left(\frac{\xi}{2}\right) \bar{J}_{n_{2} \nu}^{B}\left(-\frac{\xi}{2}\right)\right\rangle_{Q}
\end{aligned}
$$

where the auxiliary "heavy" quark fields are in the adjoint representation, and

$$
J_{B}^{\mu \nu}=F_{0, a}^{\mu \nu} Q_{0}^{a}, \quad \bar{J}_{B}^{\mu \nu}=\bar{Q}_{0}^{a} F_{0, a}^{\mu \nu} .
$$

The renormalization of $J_{B}^{\mu \nu}$ and $\bar{J}_{B}^{\mu \nu}$ is more involved than the quark case, as they can mix with other composite operators of the same or less dimensions. In DR, BRST symmetry allows $J_{B}^{\mu \nu}$ to mix with (Dorn, 1986; Zhang et al., 2019b)

$$
\begin{aligned}
& J_{2 B}^{\mu \nu}=\left(n_{z}^{\nu} F_{0, a}^{\mu n_{z}}-n_{z}^{\mu} F_{0, a}^{\nu n_{z}}\right) Q_{0}^{a} / n_{z}^{2} \\
& J_{3 B}^{\mu \nu}=\left(-i n_{z}^{\mu} A_{0, a}^{\nu}+i n_{z}^{\nu} A_{0, a}^{\mu}\right)\left[\left(i n_{z} \cdot D_{0}-i \delta m\right) Q_{0}\right]^{a} / n_{z}^{2} .
\end{aligned}
$$

Their renormalization matrix is given by (Dorn, 1986)

$$
\left(\begin{array}{l}
J_{B}^{\mu \nu} \\
J_{2 B}^{\mu \nu} \\
J_{3 B}^{\mu \nu}
\end{array}\right)=\left(\begin{array}{ccc}
Z_{11} & Z_{12} & Z_{13} \\
0 & Z_{22} & Z_{23} \\
0 & 0 & Z_{33}
\end{array}\right)\left(\begin{array}{l}
J_{R}^{\mu \nu} \\
J_{2 R}^{\mu \nu} \\
J_{3 R}^{\mu \nu}
\end{array}\right)
$$

where $J_{2 B}^{\mu \nu}$ is gauge invariant while $J_{3 B}^{\mu \nu}$ is gauge dependent and proportional to the equation of motion (EOM) for the auxiliary field. The Green's functions of the EOM operator will result in a $\delta$-function,

$$
\left(i n_{z} \cdot D_{0}(\xi)-i \delta m\right)\left\langle Q_{0}(\xi) \bar{Q}_{0}(0)\right\rangle_{Q}=\delta^{(4)}(\xi)
$$

which only contributes a contact term $\delta(z)$ after integrating over the auxiliary fields. As long as $z \neq 0$, such mixing vanishes in all Green's functions of $O_{g}^{B}(z)$, so we can ignore the mixing between $J_{B}^{\mu \nu}$ and $J_{3 B}^{\mu \nu}$ in the renormalization of $O_{g}^{B}(z)$. At $z=0, O_{g}^{B}(z)$ becomes a local operator and is known to mix with BRST-exact and EOM operators (Collins and Scalise, 1994), whose renormalization can be performed in the standard way.

Note that when contracted with $n_{z}$,

$$
\begin{aligned}
& J_{2 B}^{n_{z} \mu}=J_{B}^{n_{z} \mu}=F_{0, a}^{n_{z} \mu} Q_{0}^{a} \\
& J_{3 B}^{n_{z} \mu}=i\left(-A_{0}^{\mu, a}+\frac{n_{z}^{\mu}}{n_{z}^{2}} n_{z} \cdot A_{0}^{a}\right)\left[\left(i n_{z} \cdot D_{0}-i \delta m\right) Q_{0}\right]_{a}
\end{aligned}
$$


the $J_{B}^{n_{z} \mu}$ only mixes with the EOM operator $J_{3 B}^{n_{z} \mu}$. As has been argued above, we can ignore such mixing for $z \neq 0$. Moreover, this degeneracy also leads to relations among elements in the renormalization matrix (Dorn, 1986),

$$
Z_{11}+Z_{12}=Z_{22}, \quad Z_{13}=Z_{23} .
$$

When contracted with $n_{t}$,

$$
\begin{aligned}
J_{B}^{n_{t} \mu} & =F_{0, a}^{n_{t} \mu} Q_{0}^{a}, \\
J_{2 B}^{n_{t} \mu} & =n_{z}^{\mu} F_{a, 0}^{n_{t} n_{z}} Q_{0}^{a} / n_{z}^{2}, \\
J_{3 B}^{n_{t} \mu} & =i \frac{n_{z}^{\mu}}{n_{z}^{2}} n_{t} \cdot A_{0}^{a}\left[\left(i n_{z} \cdot D_{0}-i \delta m\right) Q_{0}\right]_{a} .
\end{aligned}
$$

As one can see, $J_{2 B}^{n_{t} \mu}$ and $J_{3 B}^{n_{t} \mu}$ vanish after contraction with $g_{\perp}^{\mu \nu}$, so $J_{B}^{n_{t} \mu}$ with transverse Lorentz index $\mu$ is multiplicatively renormalizable.

To summarize, for $z \neq 0$ and transverse Lorentz index $\mu$, both $J_{B}^{n_{z} \mu}$ and $J_{B}^{n_{t} \mu}$ are multiplicatively renormalizable in coordinate space, thus proving the renormalizability of the gluon Wilson-line operator $O_{g}^{B}(z)$,

$$
\begin{aligned}
O_{g}^{B}(z) & =Z_{J} Z_{\bar{J}} \int d^{4} \xi \delta\left(\xi^{3}-z\right) g_{\perp}^{\mu \nu}\left\langle J_{n_{1} \mu}^{R}\left(\frac{\xi}{2}\right) \bar{J}_{n_{2} \nu}^{R}\left(-\frac{\xi}{2}\right)\right\rangle_{Q} \\
& =e^{\delta m|z|} Z_{J} Z_{\bar{J}} O_{g}^{R}(z),
\end{aligned}
$$

where

$$
\begin{aligned}
& J_{B}^{n_{1} \mu}=Z_{J} J_{R}^{n_{1} \mu}=\left(Z_{Q}^{g}\right)^{\frac{1}{2}} Z_{A}^{\frac{1}{2}} Z_{V}^{g} J_{R}^{n_{1} \mu}, \\
& \bar{J}_{B}^{n_{2} \nu}=Z_{\bar{J}} J_{R}^{n_{2} \nu}=\left(Z_{Q}^{g}\right)^{\frac{1}{2}} Z_{A}^{\frac{1}{2}} Z_{\bar{V}}^{g} J_{R}^{n_{2} \mu},
\end{aligned}
$$

with $Z_{V}^{g}$ and $Z_{V}^{g}$ being the renormalization constants for the vertex involving one gluon and one "heavy quark" fields. The wavefunction renormalization constant for the auxiliary "heavy quark", $Z_{Q}^{g}$, is different from the quark case as it is in the adjoint representation.

In addition, since $J_{B}^{n_{z} \mu}$ and $J_{B}^{n_{t} \mu}$ do not mix with "heavy-to-light" quark currents due to the mismatch of quantum numbers, it implies that the nonlocal gluon Wilson-line operator does not mix with the singlet quark one under renormalization.

For the polarized gluon quasi-PDF, its definition is the same as Eq. (56), except that the gluon Wilson-line operator becomes

$$
\Delta O_{g}^{B}(z)=\epsilon_{\perp, \mu \nu} F_{0, a}^{n_{1} \mu}(z) W^{a b}(z, 0) F_{0, b}^{n_{2} \nu}(0),
$$

where $\epsilon_{\perp}^{\mu \nu}=\epsilon^{03 \mu \nu}$. Since $\epsilon_{\perp}^{\mu \nu}$ only contracts with the transverse Lorentz indices, one can use the same proof for $O_{g}^{B}(z)$ to show that $\Delta O_{g}^{B}(z)$ is also multiplicatively renormalizable and does not mix with singlet quark case (Zhang et al., 2019b).

Finally, one can also prove that Eq. (69) is valid under lattice regularization with $\delta m$ being linearly divergent (Zhang et al., 2019b). This completes the proof of renormalizability of the gluon Wilson-line operators.
One-loop renormalization. Now we demonstrate the above result by an explicit one-loop example. For the nonlocal Wilson-line operators to be multiplicatively renormalizable, it is important that all linear divergences associated with diagrams other than the Wilson line selfenergy cancel out among themselves. To see this, a gauge symmetry preserving regularization is crucial. We use DR and keep poles around $d=3$ to identify the linear divergences (Wang et al., 2019b; Zhang et al., 2019b).

The one-loop vertex correction to the "heavy-to-gluon" current is shown in Fig. 17. Each diagram contributes

$$
\begin{aligned}
I_{a}^{\rho \nu} & =\frac{\alpha_{s} C_{A}}{\pi}\left[\frac{1}{4-d} \frac{3}{4} F_{a}^{\rho \nu} Q_{a}+\text { finite terms }\right], \\
I_{b}^{\rho \nu} & =\frac{\alpha_{s} C_{A}}{\pi}\left[\frac{1}{d-4}\left(A_{a}^{\nu} n_{z}^{\rho}-A_{a}^{\rho} n_{z}^{\nu}\right) n_{z} \cdot \partial Q_{a} / n_{z}^{2}\right. \\
& \left.+\frac{\pi \mu}{d-3}\left(n_{z}^{\rho} A_{a}^{\nu}-n_{z}^{\nu} A_{a}^{\rho}\right) Q_{a}+\text { finite terms }\right], \\
I_{c}^{\rho \nu} & =\frac{\alpha_{s} C_{A}}{\pi}\left\{\frac { 1 } { d - 4 } \left[\frac{1}{2}\left(F_{a}^{\rho n_{z}} n_{z}^{\nu}-F_{a}^{\nu n_{z}} n_{z}^{\rho}\right) Q_{a} / n_{z}^{2}\right.\right. \\
& \left.+\frac{1}{4} F_{a}^{\rho \nu} Q_{a}+\frac{1}{2}\left(A_{a}^{\rho} n_{z}^{\nu}-A_{a}^{\nu} n_{z}^{\rho}\right) n_{z} \cdot \partial Q_{a} / n_{z}^{2}\right] \\
& \left.-\frac{\pi \mu}{d-3}\left(n_{z}^{\rho} A_{a}^{\nu}-n_{z}^{\nu} A_{a}^{\rho}\right) Q_{a}+\text { finite terms }\right\} .
\end{aligned}
$$

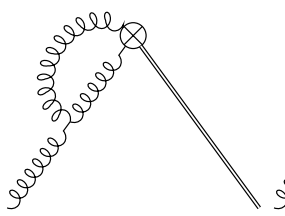

(a)

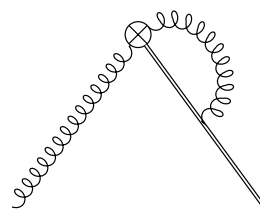

(b)

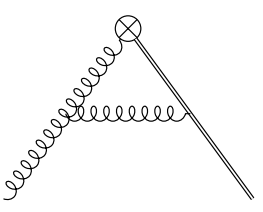

(c)
FIG. 7: One-loop vertex corrections to the "heavy-to-gluon" current.

Both Fig. 7b and Fig. 7c include a linear divergence that is evident as the $\mu /(d-3)$ term, but they cancel among themselves. This guarantees that the overall UV divergence in the vertex correction is logarithmic, thus the renormalization of the "heavy-to-gluon" current is multiplicative. Combining the one-loop results in Eq. (73) and wavefunction renormalizations, we have

$$
\begin{array}{ll}
Z_{11}=1+\frac{\alpha_{s} C_{A}}{4 \pi} \frac{1}{\epsilon_{\mathrm{UV}}}, & Z_{12}=1-\frac{\alpha_{s} C_{A}}{4 \pi} \frac{1}{\epsilon_{\mathrm{UV}}} \\
Z_{13}=Z_{23}=1-\frac{\alpha_{s} C_{A}}{4 \pi} \frac{1}{\epsilon_{\mathrm{UV}}}, & Z_{22}=0,
\end{array}
$$

where $C_{A}=N_{c}=3$ for QCD. If we ignore the mixing to the EOM operator,

$$
\begin{aligned}
& Z_{V}^{J^{n_{z \nu}}}=Z_{V}^{J^{\nu n_{z}}}=0 \\
& Z_{V}^{J^{n_{t} i}}=Z_{V}^{J^{i n_{t}}}=Z_{V}^{J^{i j}}=Z_{V}^{J^{j i}}=1+\frac{\alpha_{s} C_{A}}{4 \pi} \frac{1}{\epsilon_{\mathrm{UV}}},
\end{aligned}
$$


where $i, j=1,2$. As a result, the one-loop current renormalization constant is

$$
\begin{aligned}
Z_{J^{n z^{\nu}}} & =Z_{J^{\nu n_{z}}}=1+\frac{\alpha_{s}}{4 \pi}\left(\frac{1}{6} C_{A}-\frac{4}{3} n_{f} T_{F}\right) \frac{1}{\epsilon_{\mathrm{UV}}} \\
Z_{J^{n t^{i}}} & =Z_{J^{i n_{t}}}=Z_{J^{i j}}=Z_{J^{j i}} \\
& =1+\frac{\alpha_{s}}{4 \pi}\left(\frac{7}{6} C_{A}-\frac{4}{3} n_{f} T_{F}\right) \frac{1}{\epsilon_{\mathrm{UV}}}
\end{aligned}
$$

where $T_{F}=1 / 2$, and $n_{f}$ is the number of active quark flavors. The two-loop results can be found in (Braun et al., 2020).

As one can see, the anomalous dimension of the "heavyto-gluon" current is the same for $\mu, \nu=0,1,2$, which is due to $S O(2,1)$ (or $S O(3)$ in Euclidean space) symmetry around the $z$-axis.

\section{B. Factorization of Quasi-PDFs}

The key to LaMET applications for collinear parton physics is the factorization formula that relates the quasiPDFs to light-cone PDFs (Ji, 2013). Here we use the perturbative properties of the matching coefficients to write the factorization form in the $\overline{\mathrm{MS}}$ scheme in a way consistent with a direct EFT calculations of PDFs at any given $x$ (Izubuchi et al., 2018; Wang et al., 2019b)

$$
\begin{array}{r}
q_{i}(x, \mu)=\int_{-\infty}^{\infty} \frac{d y}{|y|}\left[\sum_{j} \tilde{C}_{q_{i} q_{j}}\left(\frac{x}{y}, \frac{\mu}{y P^{z}}\right) \tilde{q}_{j}\left(y, P^{z}, \mu\right)\right. \\
\left.\quad+\tilde{C}_{q g}\left(\frac{x}{y}, \frac{\mu}{y P^{z}}\right) \tilde{g}\left(x, P^{z}, \mu\right)\right]+\cdots, \\
g(x, \mu)=\int_{-\infty}^{\infty} \frac{d y}{|y|}\left[\sum_{j} \tilde{C}_{g q}\left(\frac{x}{y}, \frac{\mu}{y P^{z}}\right) \tilde{q}_{j}\left(y, P^{z}, \mu\right)\right. \\
\left.+\tilde{C}_{g g}\left(\frac{x}{y}, \frac{\mu}{y P^{z}}\right) \tilde{g}\left(y, P^{z}, \mu\right)\right]+\cdots,
\end{array}
$$

where $i, j$ runs over quark and anti-quark flavors. The "..." term includes mass corrections whose anayltical forms have been derived to all orders of $M^{2} /\left(P^{z}\right)^{2}$ (Chen et al., 2016), and higher-twist contributions of order $\mathcal{O}\left(\Lambda_{\mathrm{QCD}}^{2} /\left(x P^{z}\right)^{2}, \Lambda_{\mathrm{QCD}}^{2} /\left((1-x) P^{z}\right)^{2}\right)$ (see Eq. (33)). All $P^{z}$-dependence on the right hand side cancels out, just like a renormalization scale.

As we have explained in the Sec. III the above factorization is guaranteed on the physics ground because the difference between quasi-PDFs and light-cone PDFs is the order of limits in $P^{z} \rightarrow \infty$ and $\Lambda_{\mathrm{UV}} \rightarrow \infty$, and the IR physics in both quantities must be the same. An all-order factorization proof for the quark quasi-PDF in perturbation theory was first given with a diagrammatical approach (Ma and Qiu, 2018b). The formula has also been derived using the operator product expansion (OPE) of nonlocal Wilson-line operators (Izubuchi et al.,
2018; Ma and Qiu, 2018a; Wang et al., 2019b). Here we outline the diagrammatic proof similar to (Ma and Qiu, 2018b), showing that the collinear divergences of the quasi-PDFs do factorize and are equal to those of the light-cone PDFs. Since the collinear divergence is a concept in perturbation theory, we will show the factorization using a massless external quark state with lightlike momentum $P^{\mu}=\left(P^{z}, 0,0, P^{z}\right)$. While the proof is only for perturbative free quark states, the factorization formulas are widely believed to be true nonperturbatively as well. We use DR to regulate both UV and collinear divergences and only consider bare quantities, since UV renormalization does not change the leading collinear divergences.

Before the analysis, we should mention that all the soft divergences cancel between the real and virtual contributions to the quasi-PDFs, as discussed in Sec. II.B. thus we only need to focus on the collinear divergences. To obtain an intuitive understanding of the structure for collinear divergences, we start from the one-loop diagram in Fig. 5a in the Feynman gauge. The integral reads

$$
\int \frac{d^{4-2 \epsilon} k}{(2 \pi)^{4-2 \epsilon}} \frac{\operatorname{tr}\left[\not P \not k \gamma^{z} \not k\right] \delta\left(k^{z}-y P^{z}\right)}{\left(k^{2}+i 0\right)^{2}\left((P-k)^{2}+i 0\right)} .
$$

The internal quark momentum is $k^{\mu}=\left(k^{+}, k^{-}, \vec{k}_{\perp}\right)$ and the gluon momentum is $P-k$. When $k^{-}$and $k_{\perp}=\left|\vec{k}_{\perp}\right|$ are very small, the internal quark and gluon become collinear to the external quark, i.e. $k^{\mu} \sim\left(k^{+}, 0,0_{\perp}\right)$ and $(P-k)^{\mu} \sim\left(P^{+}-k^{+}, 0,0_{\perp}\right)$. In this case, the denominator of the quark and gluon propagators, $\left(k^{2}\right)^{2}$ and $(P-k)^{2}$, both vanish, which leads to collinear divergence. Conversely, for $k^{2}=(P-k)^{2}=0, k$ must be collinear to $P$ since the condition requires $k^{2}=k \cdot P=P^{2}=0$. For small $k^{-}$and $k_{\perp}$, the $\delta$ function is dominated by the $k^{+}$term of $k^{z}=\left(k^{+}-k^{-}\right) / \sqrt{2}$ and reduces to $\sqrt{2} \delta\left(k^{+}-y P^{+}\right)$. This is just the vertex which restricts $k^{+}=y P^{+}$for the light-cone PDF, up to the factor $\sqrt{2}$. Furthermore, for collinear $k$ and $(P-k)$, the spinor trace in the numerator is dominated by the $\gamma^{+}$part of $\gamma^{z}=\left(\gamma^{+}-\gamma^{-}\right) / \sqrt{2}, \operatorname{tr}\left[\not \not k \gamma^{z} \not k\right] \sim \operatorname{tr}\left[\not P \not k \gamma^{+} \not k\right] / \sqrt{2}$. Thus in the collinear region $k^{\mu} \sim\left(k^{+}, 0,0,0\right)$ the above integral reduces to that for the light-cone PDF:

$$
\int_{\mathrm{c}} \frac{d^{4-2 \epsilon} k}{(2 \pi)^{4-2 \epsilon}} \frac{\operatorname{tr}\left[\not P \not k \gamma^{+} \not k\right] \delta\left(k^{+}-y P^{+}\right)}{\left(k^{2}+i 0\right)^{2}\left((P-k)^{2}+i 0\right)}
$$

where the subscript "c" denotes the collinear region.

The above picture naturally arises in a highly boosted hadron state where the quark is approximately onshell. Therefore, as explained in Sec. II.E although the operator contains no light-cone information, the large-momentum external hadron state can still generate collinear divergences equivalent to those in the light-cone PDFs. By subtracting the full integral for light-cone PDF from that for the quasi-PDF, the logarithmic collinear divergence 
cancels, and the remaining difference is perturbative and can be absorbed into the matching kernel.

Similarly, for the vertex diagram in Fig. $5 \mathrm{~b}$, the loop integral is proportional to

$$
\int \frac{d^{4-2 \epsilon} k}{(2 \pi)^{4-2 \epsilon}} \frac{1}{P^{z}-k^{z}} \frac{\operatorname{tr}\left[\not P \gamma^{z} \not k \gamma^{z}\right] \delta\left(k^{z}-y P^{z}\right)}{\left(k^{2}+i 0\right)\left((P-k)^{2}+i 0\right)} .
$$

The whole integral in the collinear region reduces to

$$
\int_{\mathrm{c}} \frac{d^{4-2 \epsilon} k}{(2 \pi)^{4-2 \epsilon}} \frac{1}{P^{+}-k^{+}} \frac{\operatorname{tr}\left[\not P \gamma^{+} \not k \gamma^{+}\right] \delta\left(k^{+}-y P^{+}\right)}{\left(k^{2}+i 0\right)\left((P-k)^{2}+i 0\right)},
$$

which is the corresponding integral for the light-cone PDF. One key feature of the diagram is that while the gauge link probes the $z$-component of the gluon field $A^{z}=\left(A^{+}-A^{-}\right) / \sqrt{2}$, only the $A^{+}$component (longitudinal polarization) contributes to the leading collinear divergence. While attaching a new collinear gluon to the gauge-link induces a power suppression from the link propagator of $\mathcal{O}\left(1 / P^{z}\right)$, the $A^{+}$component of the collinear gluon radiated from fast-moving color charges receives enhancement from Lorentz boost factor $\gamma$ that compensates for the suppression.

The above result can be generalized to all orders. Similar to the one-loop diagrams, in the leading region of collinear divergence there are an arbitrary number of longitudinally polarized $A^{+}$gluons, which are emitted dynamically from the fast-moving state instead of being put in by hand using the lightlike gauge link, in contrast to the standard collinear PDF. The existence of the $A^{+}$ gluons clearly increases the level of complication in showing the equivalence of collinear divergences between the quasi- and light-cone PDFs. For simplification, from now on we choose to work in the light-cone gauge $A^{+}=0$ to eliminate all the $A^{+}$gluons. Therefore, the vertex diagrams no longer contribute to the leading collinear divergence, thus making its structure much simpler.

In a general diagram, we decompose the potential leading region of the quasi-PDF into the ladder structure shown in Fig. 8. The upper two-particle-irreducible (2PI) kernel that contains the nonlocal operator defining the quasi-PDF is $H$. The 2PI kernel in the ladder is $K . K$ contains the upper two external quark lines but not the lower ones. The momentum flowing out of the ladders are labeled as $k_{1}$ to $k_{n}$ from bottom to top when there are $n$ 2PI kernels. We write $H$ and $K$ as matrices in spinor and momentum space. $H=H_{\alpha^{\prime} \beta^{\prime}}\left(y P^{z} ; k\right)$ where $k$ denotes the momentum flowing into $H$ and $K=K_{\alpha \beta ; \alpha^{\prime} \beta^{\prime}}\left(k, k^{\prime}\right)$ where $k, k^{\prime}$ are the momenta of the upper and lower external legs, respectively. Here $\alpha \beta$ and $\alpha^{\prime} \beta^{\prime}$ are the spinor indices for the upper and lower two external legs, respectively. Following the method in (Collins, 2011a; Curci et al., 1980), we find that:

1. There are no collinear divergences in the upper part $H$ in the light-cone gauge.

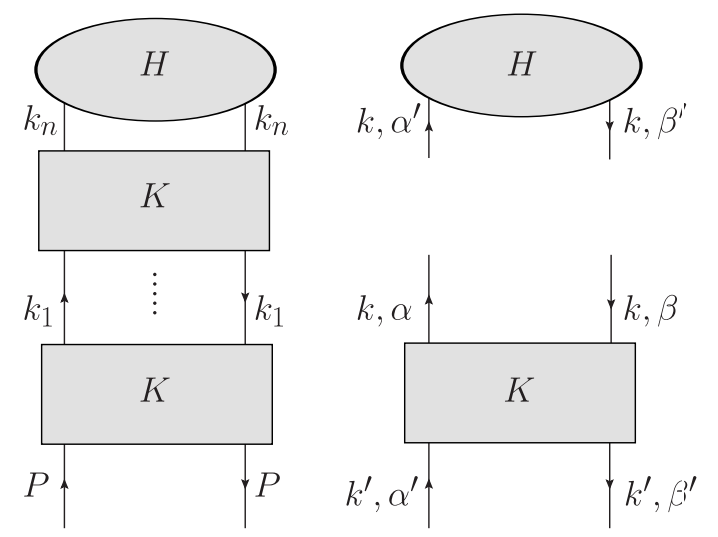

FIG. 8: The ladder decomposition of the quasi-PDF (left). The upper 2PI kernel $H$ contains the operator defining the quasi-PDF, and external two legs at the bottom of the diagram is the external large $P^{z}$ state. The kernels $H$ and $K$ are shown on the right.

2. If none of $k_{1}, \cdots, k_{n}$ is collinear, there will be no leading collinear divergence. More generally, for the $i^{\prime}$ th 2PI kernel, if either of $k_{i-1}$ and $k_{i}$ is not collinear, then the sub-integrals inside the kernel are finite and it does not contribute to leading collinear divergence.

3. If $k_{i}$ is not collinear, then there are no collinear divergences for the upper part of the diagram above the $i$ 'th ladder.

Therefore, the collinear divergences are generated in the momentum regions $R_{i}$ in which $k_{1}$ to $k_{i}$ are collinear while $k_{i+1}$ to $k_{n}$ are not. We can construct counter terms that subtract out the collinear divergences in each of the regions $R_{i}$. For this we keep only the + component of $k_{i}$ in the convergent upper part $H K^{n-i}$ as in the oneloop example, namely $k_{i} \rightarrow\left(k_{i}^{+}, 0,0_{\perp}\right)$ in the upper part. This will clearly leave the collinear divergence unchanged. Also notice that $\left[H K^{n-i}\right]_{\alpha \beta}=H_{\alpha^{\prime} \beta^{\prime}} K_{\alpha^{\prime} \beta^{\prime} ; \alpha \beta}^{n-i}$ should be understood as a $4 \times 4$ Dirac matrix with indices $\alpha \beta$, while the lower part is $\left[K^{i} \not P\right]_{\alpha \beta}=K_{\alpha \beta ; \alpha^{\prime} \beta^{\prime}}^{i} P_{\alpha^{\prime} \beta^{\prime}}$. In the leading region of collinear divergence, $H K^{n-i}$ and $K^{i} \not P$ are proportional to $\gamma^{+}$and $\gamma^{-}$respectively. Therefore, to obtain the leading collinear divergence, we can disentangle the spinor traces for the upper and lower parts by contracting them with $\gamma^{-} / 2$ and $\gamma^{+} / 2$ separately. The only communication between them is the $k^{+}$integration. The collinear divergence is contained in the lower part

$$
q^{i}\left(x, \epsilon_{\mathrm{IR}}\right)=\int \frac{d k^{-} d^{d-2} k_{\perp}}{2(2 \pi)^{d}} \operatorname{tr}\left[\gamma^{+} K^{i}\left(x P^{+}, k^{-}, k_{\perp} ; P\right) \not P\right],
$$

where $d=4-2 \epsilon, k^{+}=x P^{+}$, and the subtraction for the region $R_{i}$ can be written effectively as a convolution

$$
\int \frac{d x}{x} \hat{C}^{n-i}\left(y, x, P^{z}\right) q^{i}\left(x, \epsilon_{\mathrm{IR}}\right),
$$


where

$$
\hat{C}^{n-i}\left(y, x, P^{z}\right)=\frac{1}{2} \operatorname{tr}\left[H K^{n-i}\left(y P^{z} ; x P^{+}, 0,0_{\perp}\right)\left(x P^{+}\right) \gamma^{-}\right]
$$

is the naive matching kernel. Here the $y$ dependence comes from the operator in $H$. However, the naive matching kernel still suffers from collinear subdivergences that need to be subtracted. This can be achieved using the subtracted matching kernels $C^{n-i}(y, x)$ defined recursively in a way similar to the BPHZ relation for UV renormalization (Collins, 2011a). Summing over $n$ and $i$, the recursive relation leads to

$$
\begin{aligned}
\tilde{q}\left(y, P^{z}, \epsilon_{\mathrm{IR}}\right) & =\sum_{n=0}^{\infty} \sum_{i=0}^{n} \int \frac{d x}{x} C^{n-i}\left(y, x, P^{z}\right) q^{i}\left(x, \epsilon_{\mathrm{IR}}\right) \\
& =\int \frac{d x}{x} C\left(y, x, P^{z}\right) q\left(x, \epsilon_{\mathrm{IR}}\right),
\end{aligned}
$$

where $\tilde{q}\left(y, P^{z}, \epsilon_{\mathrm{IR}}\right)$ is the quasi-PDF, $C\left(y, x, P^{z}\right)=$ $\sum_{n=0}^{\infty} C^{n}\left(y, x, P^{z}\right)$ is the all-order matching kernel and $q\left(x, \epsilon_{\mathrm{IR}}\right)=\sum_{i=0} q^{i}\left(x, \epsilon_{\mathrm{IR}}\right)$. Based on the definition of $q^{i}\left(x, \epsilon_{\mathrm{IR}}\right)$, it is clear that $q^{i}$ equals the light-cone PDF with $i$ PI kernels and $q$ is the full light-cone PDF with natural support $0<x<1$. The light-cone PDF $q(x)$ is independent of the operator defining the quasi-PDF, as it is only sensitive to the explicit form of the collinear divergence. The r.h.s. of Eq. (86) contains all the collinear divergences from the quasi-PDF $\tilde{q}$. Thus the matching relation for bare quantities is established. A similar matching can be written down for the renormalized quantities, where the renormalization only affects the matching kernel $C\left(y, x, P^{z}\right)$. Note that the explicit solution for $C^{n-i}\left(y, x, P^{z}\right)$, which leads to Eq. (33), can be given based on a subtraction operator defined similar to that in (Collins, 2011a). Besides, Eq. (86) can be inverted order by order in $\alpha_{s}$, thus proving Eq. (33), which can also be generalized to Eqs. (77) and (78).

Now we present the matching coefficient in the $\overline{\mathrm{MS}}$ scheme at one-loop order. The one-loop expansion of the $\overline{\mathrm{MS}}$ quasi- and light-cone PDFs in a free massless quark state with momentum $p^{\mu}=\left(p^{z}, 0,0, p^{z}\right)$ are

$$
\begin{aligned}
\tilde{q}\left(y, \mu / p^{z}, \epsilon_{\mathrm{IR}}\right) & =\tilde{q}^{(0)}(y)+\frac{\alpha_{s} C_{F}}{2 \pi} \tilde{q}^{(1)}\left(y, \mu / p^{z}, \epsilon_{\mathrm{IR}}\right), \\
q\left(x, \epsilon_{\mathrm{IR}}\right) & =q^{(0)}(x)+\frac{\alpha_{s} C_{F}}{2 \pi} q^{(1)}\left(x, \epsilon_{\mathrm{IR}}\right) .
\end{aligned}
$$

At tree level, $\tilde{q}^{(0)}(y)=q^{(0)}(y)=\delta(1-y)$. At one loop, the $\overline{\mathrm{MS}}$ quasi-PDF and its counterterm are (Izubuchi et al., 2018)

$$
\begin{aligned}
& \tilde{q}^{(1)}\left(y, \mu / p^{z}, \epsilon_{\mathrm{IR}}\right) \\
& = \begin{cases}\left(\frac{1+y^{2}}{1-y} \ln \frac{y}{y-1}+1+\frac{3}{2 y}\right)_{+(1)}^{[1, \infty]}-\frac{3}{2 y} & y>1 \\
\left(\frac{1+y^{2}}{1-y}\left[-\frac{1}{\epsilon_{\mathrm{IR}}}-\ln \frac{\mu^{2}}{4\left(p^{z}\right)^{2}}+\ln (y(1-y))\right]\right. & \\
\left.-\frac{y(1+y)}{1-y}+2 \sigma(1-y)\right)_{+(1)}^{[0,1]} & 0<y<1 \\
\left(-\frac{1+y^{2}}{1-y} \ln \frac{-y}{1-y}-1+\frac{3}{2(1-y)}\right)_{+(1)}^{[-\infty, 0]} & \\
-\frac{3}{2(1-y)} & y<0\end{cases} \\
& +\delta(1-y)\left[\frac{3}{2} \ln \frac{\mu^{2}}{4\left(p^{z}\right)^{2}}+\frac{5+2 \sigma}{2}\right] \text {, } \\
& \delta \tilde{q}^{(1)}\left(y, \mu / p^{z}, \epsilon_{\mathrm{UV}}\right)=\frac{3}{2 \epsilon_{\mathrm{UV}}} \delta(1-y)
\end{aligned}
$$

where $\epsilon_{\mathrm{IR}}$ regulates the collinear divergence, $\sigma=0$ for $\Gamma=\gamma^{t}$ and 1 for $\Gamma=\gamma^{z}$. The plus function at $y=y_{0}$ with support in a given domain $D$ is defined as

$$
\int_{D} d y[g(y)]_{+\left(y_{0}\right)}^{D} h(y)=\int_{D} d y g(y)\left[h(y)-h\left(y_{0}\right)\right]
$$

with arbitrary $g(y)$ and $h(y)$. Note that the $\overline{\mathrm{MS}}$ renormalization of the quasi-PDF actually requires a subtle treatment of vector current conservation (Izubuchi et al., 2018). We only present results in the form that is sufficient for our discussion, which differs slightly from that in (Izubuchi et al., 2018) by the $\delta$-functions at $y= \pm \infty$ and from the treatment in (Alexandrou et al., 2019b).

On the other hand,

$$
q^{(1)}\left(x, \epsilon_{\mathrm{IR}}\right)=\frac{\alpha_{s} C_{F}}{2 \pi} \frac{(-1)}{\epsilon_{\mathrm{IR}}}\left(\frac{1+x^{2}}{1-x}\right)_{+(1)}^{[0,1]},
$$

which is limited to the physical region as expected.

By comparing the quasi- and light-cone PDFs in Eqs. (89) and (92), we find that both of them have the same collinear divergence, or in other words, they share the same IR physics, thus validating the factorization formula at one-loop order. Setting $p^{z}=x P^{z}$ and plugging the one-loop results into Eq. (33), we extract the matching coefficient for the hadron matrix element which only depends on the perturbative scales $\mu$ and $P^{z}$,

$$
\begin{aligned}
& C^{\overline{\mathrm{MS}}}\left(y, \frac{\mu}{x P^{z}}\right)=\delta(1-y)+\frac{\alpha_{s} C_{F}}{2 \pi}\left[\tilde{q}^{(1)}\left(y, \frac{\mu}{x P^{z}}, \epsilon_{\mathrm{IR}}\right)\right. \\
&\left.-q^{(1)}\left(y, \epsilon_{\mathrm{IR}}\right)\right] .
\end{aligned}
$$

The complete one-loop matching coefficients in Eq. 333 in the transverse-momentum cutoff and $\overline{\mathrm{MS}}$ schemes can be found in (Wang et al., 2019b; Wang and Zhao, 2018; Wang et al., 2018). The two-loop results were obtained recently in (Chen et al., 2020a b. c; Li et al., 2020). 


\section{Coordinate-Space Factorization of Bilinear Operators}

Although the LaMET application to PDFs concerns the expansion of momentum densities in the $P^{z} \rightarrow \infty$ limit, lattice QCD calculations actually start from computing coordinate-space correlations, for example,

$$
\tilde{h}\left(z, P^{z}\right)=\frac{1}{N_{\Gamma}}\left\langle P^{z}\left|O_{\Gamma}(z)\right| P^{z}\right\rangle,
$$

at all $z$ and do Fourier transform with respect to $\lambda=z P^{z}$ at a fixed $P^{z}$. Here the normalization factor $N_{\Gamma}=2 P^{z}$ for $\Gamma=\gamma^{z}$ and $N_{\Gamma}=2 P^{t}$ for $\Gamma=\gamma^{t}$. The $\tilde{h}\left(z, P^{z}\right)$ is a function of two independent variables $z$ and $P^{z}$, and in LaMET analysis the relevant combinations are quasi-LF distance $\lambda$ (see Fig. 6) and $P^{z}$, hence $\tilde{h}\left(\lambda, P^{z}\right)$ will be called quasi-LF correlation, which is distinguished from the LF correlation $h(\lambda, \mu)$ below.

The coordinate-space factorization approach in (Braun et al., 1995) has been suggested as an alternative way to extract the PDFs from $\tilde{h}\left(z, P^{z}\right)$ (Orginos et al., 2017; Radyushkin, 2017a, 2019a), which is closely related to the OPE. Instead of working with variables $\lambda$ and $P^{z}$, one may consider $\tilde{h}$ as a function of $\lambda$ and $z^{2}$, i.e., $\tilde{h}\left(\lambda, z^{2}\right)$. The Fourier transform of $\tilde{h}\left(\lambda, z^{2}\right)$ with respect to $\lambda$ is no longer the momentum distribution of the proton at a fixed momentum. Instead, it is called a pseudo-distribution (Radyushkin, 2017a). At small $|z| \ll \Lambda_{\mathrm{QCD}}^{-1}, \tilde{h}\left(\lambda, z^{2}\right)$ can be factorized into the lightcone correlation (Izubuchi et al., 2018; Radyushkin, 2018a),

$$
\tilde{h}\left(\lambda, z^{2} \mu^{2}\right)=\int_{-1}^{1} d \alpha \mathcal{C}\left(\alpha, z^{2} \mu^{2}\right) h(\alpha \lambda, \mu)+\ldots,
$$

where $\ldots$ are the power corrections in $z^{2} \Lambda_{\mathrm{QCD}}^{2}$, and the matching coefficient $\mathcal{C}$ is related to $C$ in Eq. (33) by

$$
C\left(\eta, \frac{\mu}{x P^{z}}\right)=\int \frac{d \lambda}{2 \pi} e^{i \eta \lambda} \int_{-1}^{1} d \alpha e^{-i \lambda \alpha} \mathcal{C}\left(\alpha, \frac{\mu^{2} \lambda^{2}}{\left(x P^{z}\right)^{2}}\right)
$$

To illustrate the connection between the above factorization in Eq. (95) and OPE, let us take the nonsinglet quark case as an example (Izubuchi et al., 2018; Wang et al., 2019b). In the $\overline{\mathrm{MS}}$ scheme, the renormalized $O_{\gamma^{\mu_{0}}}(z, \mu)$ can be expanded in terms of local gaugeinvariant twist- 2 operators as $z^{2} \rightarrow 0$,

$$
\begin{aligned}
O_{\gamma^{\mu_{0}}}(z, \mu)=\sum_{n=0}^{\infty} & {\left[C_{n}\left(\mu^{2} z^{2}\right) \frac{(i z)^{n}}{n !}\left(n_{z}\right)_{\mu_{1}} \cdots\left(n_{z}\right)_{\mu_{n}}\right.} \\
& \left.\times O^{\mu_{0} \mu_{1} \cdots \mu_{n}}(\mu)+\text { higher-twist }\right]
\end{aligned}
$$

where $\mu_{0}=0$ or $3, C_{n}=1+\mathcal{O}\left(\alpha_{s}\right)$ is the Wilson coefficient, and $O^{\mu_{0} \mu_{1} \cdots \mu_{n}}(\mu)$ is the twist-two operator in Eq. (14).

Using the hadron matrix elements in Eq. (15) and their relation to the light-cone PDF in Eq. (16), we write down the small- $|z|$ expansion of the hadron matrix element of $O_{\gamma^{\mu_{0}}}(z, \mu)$ (Izubuchi et al., 2018),

$$
\begin{aligned}
\tilde{h}\left(\lambda, z^{2} \mu^{2}\right)= & \left\langle P\left|O_{\gamma^{\mu_{0}}}(z, \mu)\right| P\right\rangle /\left(2 P^{\mu_{0}}\right) \\
= & \sum_{n=0}^{\infty} C_{n}\left(z^{2} \mu^{2}\right) \frac{(-i \lambda)^{n}}{n !}\left[1+\mathcal{O}\left(\frac{M^{2}}{\left(P^{z}\right)^{2}}\right)\right] \\
& \times \int_{-1}^{1} d x x^{n} q(x, \mu)+\mathcal{O}\left(z^{2} \Lambda_{\mathrm{QCD}}^{2}\right),
\end{aligned}
$$

where the $\mathcal{O}\left(M^{2} /\left(P^{z}\right)^{2}\right)$ term comes from the kinematic trace contribution and the $\mathcal{O}\left(z^{2} \Lambda_{\mathrm{QCD}}^{2}\right)$ term from higher-twist. The Wilson coefficients $C_{n}\left(z^{2} \mu^{2}\right)$ have been calculated at one-loop (Izubuchi et al., 2018) and twoloop (Li et al., 2020) orders. Comparing the above equation with Eq. (95), we identify

$$
\mathcal{C}\left(\alpha, \mu^{2} z^{2}\right) \equiv \int \frac{d \lambda}{2 \pi} e^{i \lambda \alpha} \sum_{n} C_{n}\left(\mu^{2} z^{2}\right) \frac{(-i \lambda)^{n}}{n !} .
$$

Since $z^{2}$ is fixed in $\mathcal{C}\left(\alpha, \mu^{2} z^{2}\right)$, the integration in Eq. (99) is actually over $P^{z}$ from $-\infty$ to $+\infty$. $\mathcal{C}\left(\alpha, z^{2} \mu^{2}\right)$ has support $-1 \leq \alpha \leq 1$, and its one-loop result is

$$
\begin{aligned}
& \mathcal{C}\left(\alpha, z^{2} \mu^{2}\right) \\
= & {\left[1+\frac{\alpha_{s} C_{F}}{2 \pi}\left(\frac{3}{2} \ln \frac{z^{2} \mu^{2} e^{2 \gamma_{E}}}{4}+\frac{3}{2}\right)\right] \delta(1-\alpha) } \\
& +\frac{\alpha_{s} C_{F}}{2 \pi}\left\{\left(\frac{1+\alpha^{2}}{1-\alpha}\right)_{+(1)}^{[0,1]}\left[-\ln \frac{z^{2} \mu^{2} e^{2 \gamma_{E}}}{4}-1\right]\right. \\
& \left.-\left(\frac{4 \ln (1-\alpha)}{1-\alpha}\right)_{+(1)}^{[0,1]}+2(1+\sigma)(1-\alpha)\right\} \theta(\alpha) \theta(1-\alpha),
\end{aligned}
$$

which was also calculated and further studied in (Ji et al., 2017b; Li et al., 2020; Radyushkin, 2018a,b; Zhang et al., 2018). One can check that the above result is indeed related to one-loop momentum-space matching by Eq. (96). Since we are interested in the relation between the quasi-LF correlation with the matrix element of the light-ray operator $O_{\gamma^{+}}(\lambda n)$, Eq. (95) can also be obtained by using the light-ray operator expansion in Balitsky and Braun, 1989; Braun et al., 1995; Braun and Müller, 2008).

Using OPE or short-distance expansion, the exact factorization formula for the gluon and singlet quark quasiPDFs, which includes their mixings, has also been derived in coordinate space and studied at one-loop order (Balitsky et al., 2019; Wang et al., 2019b).

It is easy to see that the limits $P^{z} \rightarrow \infty$ in LaMET expansion and $z \rightarrow 0$ in coordinate-space factorization, keeping finite $\lambda=z P^{z}$, are equivalent. However, in practical lattice QCD calculations, one is limited by the largest momentum $P_{\max }^{z}$ in a specific setup, and the two approaches start to differ.

In LaMET systematic approximation, one should calculate $\tilde{h}\left(z, P_{\max }^{z}\right)$ with all possible $z$ or $\lambda$, but in prac- 
tice the largest $\lambda_{\max }=z_{\max } P_{\max }^{z}$ is limited by the lattice volume as well as data quality at large $z$. Due to QCD confinement, $\tilde{h}\left(z, P_{\max }^{z}\right)$ has a correlation length $\sim 1 / \Lambda_{\mathrm{OCD}}$, leading to an exponential decay at large $z$ (Ji et al., 2020b). Therefore, if $z_{\max }$ is sufficiently large (e.g., the proton size $\sim 1 \mathrm{fm})$ for $\tilde{h}\left(z, P_{\max }^{z}\right)$ to fall to almost zero, then the truncated Fourier transform of $\tilde{h}\left(z, P_{\max }^{z}\right)$ should converge quickly, and the truncation effects mainly affect results at small $x \lesssim 1 / \lambda_{\max }$. If $\tilde{h}\left(z, P_{\max }^{z}\right)$ exhibits exponential decay but still has a nonzero value at $z_{\max }$, then one can perform a physically motivated extrapolation beyond $z_{\max }$ (Ji et al., 2020b) to do the Fourier transform, which removes the unphysical oscillation from truncation and only affects the small- $x$ region. In the momentum space, one can use LaMET expansion to calculate the PDF point by point in $x$ with systematic error controlled by $\Lambda_{\mathrm{QCD}}^{2} /\left(x P_{\max }^{z}\right)^{2}$ and $\Lambda_{\mathrm{QCD}}^{2} /\left((1-x) P_{\max }^{z}\right)^{2}$, which gives the prediction for a cetain region of $x,\left[x_{\min }, x_{\max }\right]$, with a target error.

In coordinate-space factorization, one expands $\tilde{h}\left(\lambda, z^{2}\right)$ in $z^{2} \Lambda_{\mathrm{QCD}}^{2}$. For the factorization formula to be valid, $z$ must remain in the perturbative region. For example, an estimate in (Ji et al., 2020b) gives $z_{\max } \sim 0.3-$ $0.4 \mathrm{fm}$. Although there have been observations that forming ratios of $\tilde{h}\left(\lambda, z^{2}\right)$ may cancel the higher-twist contributions at $z>0.4 \mathrm{fm}$ (Orginos et al., 2017), this cancellation needs be quantified for precision calculations. With a finite range of quasi-LF correlations, the PDFs can be extracted through modelling the $x$-dependence or more advanced techniques such as Bayesian analysis (Bringewatt et al., 2020) or neural network (Cichv et al., 2019; Del Debbio et al., 2020a; Karpie et al., 2019), which is similar to extracting the PDFs from experimental data ( $\mathrm{Ma}$ and Qiu, 2018a), although quantifying the systematic error from fitting can be challenging. The coordinate-space factorization can also provide the extraction of the lowest moments of PDFs (Gao et al., 2020; Joó et al., 2020; Karpie et al., 2018; Shugert et al., 2020), where the main systematic error is controlled by $z^{2} \Lambda_{\mathrm{QCD}}^{2}$.

So far, there have been very limited studies about the comparison between quasi- and pseudo-PDF analysis (Alexandrou et al., 2020c; Bhat et al., 2020). It remains to be seen how systematic errors in the two strategies are compared and contrasted.

\section{Nonperturbative Renormalization and Matching}

The multiplicative renormalizability of the nonlocal Wilson-line operators for quasi-PDFs allows a nonperturbative renormalization on the lattice, after which the continuum limit can be taken. This is an important step in the application of LaMET. One way of doing so is to perform a mass subtraction of the Wilson line first (Chen et al., 2017; Green et al.,
2018, 2020; Ishikawa et al., 2016; Musch et al., 2011; Zhang et al., 2017), and then renormalize the remnant UV divergences with lattice perturbation theory or other nonperturbative schemes. Another scheme which has gained more popularity in recent years is the regularization-independent momentum subtraction (RI/MOM) scheme (Alexandrou et al., 2017b; Chen et al., 2018; Constantinou and Panagopoulos, 2017; Liu et al., 2020; Stewart and Zhao, 2018). In the coordinate space approach where $|z| \ll \Lambda_{\mathrm{QCD}}^{-1}$, the ratios of quasi-LF correlations in different states (Braun et al., 2019; Li et al., 2020; Orginos et al., 2017; Radyushkin, 2017a) have also been proposed as a renormalization scheme. At large $z$, the $\mathrm{RI} / \mathrm{MOM}$ and ratio schemes introduce extra nonperturbative effects at different levels, which may distort the IR property of the original quasi-LF correlations. Due to the suppression of long-range contributions by large $P^{z}$ in the Fourier transform, this nonperturbative contamination mainly affects the end-point region in $x$-space, while the existing LaMET calculations with RI/MOM scheme at moderate $x$, for example in (Alexandrou et al., 2018b; Lin et al., 2018a), suffers less from such systematics. Nevertheless, the above complication can be avoided by switching to the hybrid scheme (Ji et al., 2020b) where one utilizes the advantages of different schemes at short and large distances. In the following, we discuss the above schemes in order, with a particular focus on the hybrid renormalization scheme.

Before we proceed, it should be noted that the current-current correlators in (Braun and Müller, 2008; Detmold and Lin, 2006; Ma and Qiu, 2018a) do not need or have simple renormalization on the lattice, though it might be more costly to simulate them. Besides, there is another distinct method based on a redefinition of the quasi-PDF with smeared fermion and gauge fields via the gradient flow (Monahan and Orginos, 2017). The smeared quasi-PDF is free from UV divergences and remains finite in the continuum limit, which can be perturbatively matched onto the PDF (Monahan, 2018b). Nevertheless, this method awaits to be implemented on the lattice.

\section{Wilson-line mass-subtraction scheme}

Since the mass correction $\delta m$ includes all the linear UV divergences, it is highly favored to nonperturbatively subtract it from the quasi-PDFs. It is well known that the Wilson line renormalization is related to the additive renormalization of the static quark-antiquark potential, i.e., $\delta m$, especially in the context of finite temperature field theory. For a rectangle-shaped Wilson loop of dimension $L \times T$ in the spatial and temporal directions, its 
vacuum expectation value for large $T$ scales as

$$
\lim _{T \rightarrow \infty} W(L, T)=c(L) e^{-V(L) T}
$$

The renormalized static potential is

$$
V^{R}(L)=V(L)+2 \delta m,
$$

and $\delta m$ can be fixed by imposing the condition $V^{R}\left(L_{0}\right)=$ 0 for a particular value of $L_{0}$. Alternatively, one can also fit $\delta m$ from the famous string potential model,

$$
V(L)=\sigma L-\frac{\pi}{12 L}-2 \delta m
$$

Apart from using the static potential to determine $\delta m$, it was also proposed to calculate this quantity in the auxiliary "heavy quark" field theory with the following condition (Green et al., 2018),

$$
\delta m=\left.\frac{d}{d z} \ln \operatorname{Tr}\left\langle Q\left(x+z n_{z}\right) \bar{Q}(x)\right\rangle_{\mathrm{QCD}+Q}\right|_{z=z_{0}}
$$

Other suggestions have also been made for a nonperturbative calculation of $\delta m$ (Ji et al., 2020b). For example, one can investigate the asymptotic large- $z$ behavior of the hadron matrix element or the single quark Green's function, of the vacuum expectation value of $O_{\Gamma}(z, a)$ in a fixed gauge. The $\delta m$ calculated from all these matrix elements will have the following dependence on the lattice spacing $a$,

$$
\delta m=m_{-1}(a) / a+m_{0},
$$

where $m_{-1}(\mathrm{a})$ is the coefficient of the power divergence which is independent of the specific matrix element, while $m_{0} \sim O\left(\Lambda_{\mathrm{QCD}}\right)$ is finite and depends on the external state. The determination of $m_{0}$ can be rather nontrivial, and in practical calculations one could adopt a finetuning method, such as that for the Wilson-fermion mass, to find the critical value of $m_{0}$ at which the final result converges fastest in the large $P^{z}$ limit.

After the Wilson-line mass subtraction, there are still logarithmic UV divergences in $O_{\Gamma}(z, a)$. One can use lattice perturbation theory to match $\delta m$-subtracted $O_{\Gamma}(z, a)$ to the $\overline{\mathrm{MS}}$ scheme (Constantinou and Panagopoulos, 2017; Ishikawa et al., 2016; Xiong et al., 2017), but the convergence still needs to be examined at higher orders. In (Green et al., 2018, 2020), the logarithmic divergences were nonperturbatively renormalized with RI/MOM-like schemes.

The Wilson-line mass-subtraction has been implemented on the lattice in (Alexandrou et al., 2020c; Green et al., 2018; Musch et al., 2011; Zhang et al., 2017, 2019c).

\section{RI/MOM scheme}

The RI/MOM scheme has been widely used in lattice QCD for the renormalization of local composite quark operators that are free from power-divergent mixings (Martinelli et al., 1995). It is essentially a momentum subtraction scheme in QFT and can be nonperturbatively implemented on the lattice. For an arbitrary composite quark bilinear operator $O^{B}$ that is multiplicatively renormalized as $O^{B}=Z_{O} O^{R}$, the RI/MOM scheme is defined by imposing the following condition on its off-shell quark matrix element at a subtraction scale $\mu_{R}$,

$$
\left.Z_{O}^{-1}\left\langle p\left|O^{B}\right| p\right\rangle\right|_{p^{2}=-\mu_{R}^{2}}=\langle p|O| p\rangle_{\text {tree }}
$$

where the subscript "tree" means the tree-level matrix element in perturbation theory. If $\mu_{R} \gg \Lambda_{\mathrm{QCD}}, Z_{O}$ defined in Eq. (106) is in the perturbative region, and we can convert it to the $\overline{\mathrm{MS}}$ scheme order by order in perturbation theory. In this sense, $Z_{O}$ is not literally nonperturbative, but an all-order calculable quantity.

Since the nonlocal quark bilinear operator $O_{\Gamma}(z)$ has been proven to be multiplicatively renormalizable in the coordinate space, one can also renormalize it in the $\mathrm{RI} / \mathrm{MOM}$ scheme and then match the result to PDF in the $\overline{\mathrm{MS}}$ scheme (Constantinou and Panagopoulos, 2017; Stewart and Zhao, 2018). On the lattice, the off-shell matrix element of an operator is defined from its amputated Green's function, or vertex function, with off-shell quarks. For the nonlocal Wilson-line operator, the latter is

$$
\begin{aligned}
& \Lambda_{0}^{\Gamma}(z, a, p) \equiv\left[S_{0}^{-1}(p, a)\right]^{\dagger} \sum_{x, y} e^{i p \cdot(x-y)} \\
& \quad \times\left\langle 0\left|T\left[\psi_{0}(x, a) O_{\Gamma}^{B}(z, a) \bar{\psi}_{0}(y, a)\right]\right| 0\right\rangle S_{0}^{-1}(p, a),
\end{aligned}
$$

where $S_{0}(p, a)$ is the bare quark propagator, and the external momentum $p$ is Euclidean on the lattice. Since Green's functions are not gauge invariant, one needs to fix a gauge (usually Landau gauge $\partial \cdot A=0$ is chosen), and the gauge dependence is expected to be canceled by the matching or scheme conversion order by order in perturbation theory.

After including the quark wavefunction renormalization $Z_{q}$, which can be determined independently on the lattice (Martinelli et al., 1995), Eq. (106) is revised as

$$
\left.Z_{q} Z_{O_{\Gamma}}^{-1} \Lambda_{0}^{\Gamma}(z, a, p)\right|_{p=p_{R}}=\Lambda_{\text {tree }}^{\Gamma}(z, a, p)=\Gamma e^{i p_{R} \cdot z}
$$

Since $O_{\Gamma}(z, a)$ is not $O(4)$ covariant, one needs to define the $\mathrm{RI} / \mathrm{MOM}$ scheme with two scales, one is $\mu_{R}=\left|p_{R}\right|$, and the other $p_{R}^{z}$. For convenience we simply denote them as $p=p_{R}$. To work in the perturbative region and control the lattice discretization effects that are of order $\mathcal{O}\left(a^{2} \mu_{R}^{2}, a^{2}\left(p_{R}^{z}\right)^{2}\right)$, one must work in the window $\Lambda_{\mathrm{QCD}} \ll \mu_{R} \ll a^{-1}, p_{R}^{z} \ll a^{-1}$, which is attainable if the lattice spacing is small enough. 
Since the quarks are off-shell, also finite mixings with the EOM operators can appear. As a result, Eq. (108) in general cannot be satisfied as a matrix equation. Instead, one usually needs a projection operator $\mathcal{P}$ to define the off-shell matrix elements, i.e.

$$
\left\langle p\left|O_{\Gamma}^{B}\right| p\right\rangle=\operatorname{tr}\left[\Lambda_{0}^{\Gamma}(z, a, p) \mathcal{P}\right],
$$

so as to calculate the renormalization factor $Z_{O_{\Gamma}}$.

Then, the bare hadron matrix element $\tilde{h}_{B}\left(z, P^{z}, a\right)$ can be renormalized in coordinate space as

$$
\tilde{h}_{R}\left(z, P^{z}, p_{R}^{z}, \mu_{R}, a\right)=Z_{O}^{-1}\left(z, p_{R}^{z}, \mu_{R}, a\right) \tilde{h}_{B}\left(z, P^{z}, a\right),
$$

In the continuum limit, the renormalized matrix element is independent of the UV regulator, so we should obtain the same result in DR under RI/MOM scheme, i.e.,

$$
\begin{gathered}
\tilde{h}_{R}\left(z, P^{z}, p_{R}^{z}, \mu_{R}\right)=\lim _{a \rightarrow 0} \tilde{h}_{R}\left(z, P^{z}, p_{R}^{z}, \mu_{R}, a\right) \\
=\lim _{\epsilon \rightarrow 0} Z_{O}^{-1}\left(z, p_{R}^{z}, \mu_{R}, \epsilon\right) \tilde{h}_{B}\left(z, P^{z}, \epsilon\right),
\end{gathered}
$$

which allows us to compute the matching coefficients in continuum perturbation theory. Note that $\delta m$ vanishes in $Z_{O}$ due to the use of DR.

By Fourier transforming the above renormalized matrix element to momentum space, one can then work out the RI/MOM matching coefficient for the quasiPDFs (Stewart and Zhao, 2018). The one-loop matching coefficient for different spin structures has been obtained in (Liu et al., 2018, 2020; Stewart and Zhao, 2018), and the two-loop result for the unpolarized case can be found in (Chen et al., 2020b). Alternatively, one can also first convert the $\mathrm{RI} / \mathrm{MOM}$ matrix element to the $\overline{\mathrm{MS}}$ or modified $\overline{\mathrm{MS}}$ schemes (Alexandrou et al., 2019b; Constantinou and Panagopoulos, 2017), and then do the Fourier transform and momentum-space matching.

\section{Ratio scheme}

In the coordinate-space factorization, $|z| \ll \Lambda_{\mathrm{QCD}}^{-1}$ must be small, whereas $P^{z}$ can be of any value. In this case, the ratio scheme in (Orginos et al., 2017; Radyushkin, 2017a) can be an effective choice for lattice renormalization. Consider the ratio

$$
\tilde{h}\left(\lambda, z^{2}, a\right) / \tilde{h}\left(0, z^{2}, a\right),
$$

where the denominator is a nonperturbative matrix element at $P^{z}=0$. Since $\tilde{h}\left(\lambda, z^{2}, a\right)$ and $\tilde{h}\left(0, z^{2}, a\right)$ calculated from the same lattice ensemble are correlated with each other, the error in the ratio can be reduced. Besides, the ratio does not need further renormalization on the lattice, so one can directly take the continuum limit

$$
\lim _{a \rightarrow 0} \frac{\tilde{h}\left(\lambda, z^{2}, a\right)}{\tilde{h}\left(0, z^{2}, a\right)}=\frac{\tilde{h}\left(\lambda, z^{2}\right)}{\tilde{h}\left(0, z^{2}\right)},
$$

which has referred to as the "reduced Ioffe-time pseudo" distribution in (Orginos et al., 2017; Radyushkin, 2017a). In the $\overline{\mathrm{MS}}$ scheme, $\tilde{h}\left(0, z^{2} \mu^{2}\right)$ has a small- $z$ expansion,

$$
\tilde{h}\left(0, z^{2} \mu^{2}\right)=C_{0}\left(z^{2} \mu^{2}\right)+\mathcal{O}\left(z^{2} M^{2}, z^{2} \Lambda_{\mathrm{QCD}}^{2}\right),
$$

where the lowest moment of the iso-vector quark PDF $a_{0}$ is trivially one. If we ignore all the power corrections, then $\tilde{h}\left(0, z^{2} \mu^{2}\right)$ is perturbative and can be regarded as a renormalization factor. Therefore, the ratio in Eq. (113) still satisfies a similar OPE or factorization formula to Eqs. (98) and (95), except that the matching coefficient must be modified correspondingly (Izubuchi et al., 2018; Radyushkin, 2018a),

$$
\mathcal{C}^{\text {ratio }}\left(\alpha, z^{2} \mu^{2}\right)=\mathcal{C}\left(\alpha, z^{2} \mu^{2}\right)-\delta(1-\alpha) C_{0}\left(z^{2} \mu^{2}\right)
$$

In other variants of the ratio scheme, it has also been suggested that one replaces $\tilde{h}\left(0, z^{2}, a\right)$ by the vacuum matrix element of the nonlocal Wilson line operator (Braun et al., 2019; Li et al., 2020), as the UV divergence does not depend on the external state.

\section{Hybrid scheme}

Since the factorization formula for the quasi-PDF is only proven in the $\overline{\mathrm{MS}}$ scheme, it is not legitimate to use momentum-space factorization for any other scheme that differ from $\overline{\mathrm{MS}}$ nonperturbatively. The RI/MOM and ratio schemes fall into this category as the conversion factors that match them to $\overline{\mathrm{MS}}$ includes logarithms of $z^{2}$ (Constantinou and Panagopoulos, 2017; Izubuchi et al., 2018), which requires running $\alpha_{s}$ to the IR region when $z \sim \Lambda_{\mathrm{QCD}}^{-1}$. In contrast, the Wilson-line mass-subtraction scheme with wavefunction renormalizations is essentially the same as $\overline{\mathrm{MS}}$, so it will not introduce extra IR effects.

However, the Wilson-line mass-subtraction scheme also has disadvantages. On the lattice, due to discretization effects at $z \sim a$, the lattice scheme cannot reproduce the short-distance $\ln z^{2}$ behavior of the $\overline{\mathrm{MS}}$ matrix elements of the nonlocal operator. Such discretization effects, however, are cancelled in the RI/MOM or ratio scheme. To take advantages of both types of schemes, the hybrid scheme was proposed in (Ji et al., 2020b) which provides a viable approach to renormalize the quasi-LF correlations at all $z$.

To begin with, for $|z| \leq z_{\mathrm{S}}$ where $z_{\mathrm{S}}$ is smaller than the distance at which the leading-twist approximation in the OPE becomes unreliable, one renormalizes the quasi-LF correlation as

$$
\frac{\tilde{h}\left(z, a, P^{z}\right)}{Z_{X}(z, a)},
$$

where " $X$ " can be the RI/MOM or ratio scheme. 
For $|z|>z_{\mathrm{S}}$, one applies the Wilson-line mass subtraction

$$
\tilde{h}\left(z, a, P^{z}\right) e^{-\delta m|z|} Z_{\text {hybrid }},
$$

where $Z_{\text {hybrid }}$ denotes the wavefunction and vertex renormalizations, which can be nonperturbatively determined by imposing a continuity condition at $z=z_{\mathrm{S}}$,

$$
Z_{\text {hybrid }} e^{-\delta m\left|z_{\mathrm{S}}\right|} \tilde{h}\left(z, a, P^{z}\right)=\frac{\tilde{h}\left(z, a, P^{z}\right)}{Z_{X}\left(z_{\mathrm{S}}, a\right)},
$$

leading to

$$
Z_{\text {hybrid }}\left(z_{\mathrm{S}}, a\right)=e^{\delta m\left|z_{\mathrm{S}}\right|} / Z_{X}\left(z_{\mathrm{S}}, a\right) .
$$

In this way, one only has to calculate $\delta m$. Note that the final result should be independent of $z_{\mathrm{S}}$, so one should try multiple values and find the optimal one around which the result changes the most slightly.

The perturbative matching for the hybrid renormalized quasi-PDF can be derived accordingly. Taking $Z_{X}$ being the zero-momentum matrix element in the ratio scheme as an example, the $O\left(\alpha_{s}\right)$ matching has been derived as (Ji et al., 2020b)

$$
\begin{gathered}
C_{\text {hybrid }}\left(\xi, \mu^{2} /\left(p^{z}\right)^{2}, z_{\mathrm{S}}^{2} \mu^{2}\right)=C_{\text {ratio }}\left(\xi, \mu^{2} /\left(p^{z}\right)^{2}\right) \\
+\frac{\alpha_{s} C_{F}}{2 \pi} \frac{3}{2}\left[-\frac{1}{|1-\xi|_{+}}+\frac{2 \operatorname{Si}\left((1-\xi) \lambda_{\mathrm{S}}\right)}{\pi(1-\xi)}\right]
\end{gathered}
$$

where $C_{\text {ratio }}$ can be found in (Izubuchi et al., 2018), $\xi=$ $y / x$, and $\lambda_{\mathrm{S}}=z_{\mathrm{S}} p^{z}$ with $p^{z}=x P^{z}$ being the parton momentum. The plus function is defined as

$$
\frac{1}{|1-\xi|_{+}} \equiv \lim _{\beta \rightarrow 0^{+}}\left[\frac{\theta(|1-\xi|-\beta)}{|1-\xi|}+2 \delta(1-\xi) \ln \beta\right] .
$$

Due to finite lattice volume and deteriorating signal-tonoise ratios at large $z$, the available lattice data have to be truncated at $z_{\mathrm{L}}$. As we have discussed in Sec. III.C, the quasi-LF correlation has a correlation length $\xi_{z} \sim \Lambda_{\mathrm{QCD}}^{-1}$ and exhibits an exponential decay at large $z(\sim 1 \mathrm{fm})$. If $z_{\mathrm{L}}$ is not sufficiently large and the quasi-LF correlation still has a considerable nonzero value, then a direct Fourier transform truncated at $z_{\mathrm{L}}$ will lead to unphysical oscillations and other systematics in the quasi-PDF.

To improve this situation, it is suggested in the hybrid scheme to perform an extrapolation to $z \rightarrow \infty$ (Ji et al., $2020 \mathrm{~b})$. When $P^{z}$ is not very large and the lattice matrix elements exhibit the exponential behavior near $z_{\mathrm{L}}$, one can use the form $\sim e^{-z / \xi_{z}}$ to do the extrapolation, although some algebraic behavior can be added on top to better reflect the $z$-dependence. If $P^{z}$ is very large, then the signal-to-noise ratio gets worse, so $z_{\mathrm{L}}$ is smaller. In this case, the quasi-LF correlation is yet to show exponential decay and dominated by the leading-twist contributions, so one can use the algebraic decay form to do the extrapolation. Since $\lambda_{\mathrm{L}}=z_{\mathrm{L}} P^{z}$ can reach reasonably large values with contemporary computing resources, the extrapolation will only affect very small- $x$ region, for which the LaMET expansion is not well under control after all.

To summarize, the hybrid scheme provides a proper renormalization of the quasi-LF correlations at all $z$, which allows for a controlled calculation of the PDF for $x \in\left[x_{\min }, x_{\max }\right]$ through LaMET expansion in momentum space. Therefore, we expect it to play a dominant role in the LaMET calculation of PDFs in the future.

\section{E. Total Gluon Helicity $\Delta G$ and Transversity PDF}

Apart from the collinear PDFs, the first application of LaMET is the gluon helicity contribution $\Delta G$ to the proton spin (Ji et al., 2013b). In the naive sum rule for the proton spin (Jaffe and Manohar, 1990), $\Delta G$ is related to the matrix element of a nonlocal light-cone correlation operator (Manohar, 1991),

$\Delta G=\left\langle P S\left|i \int \frac{d x d \lambda}{2 \pi x P^{+}} e^{i \lambda x} F^{+\alpha}(0) W(0, \lambda n) \tilde{F}_{\alpha}{ }^{+}(\lambda n)\right| P S\right\rangle$,

which in the light-cone gauge $A^{+}=0$ reduces to

$$
\Delta G=\left\langle P S\left|(\vec{E} \times \vec{A})^{z}\right| P S\right\rangle /\left(2 P^{+}\right) .
$$

Within the LaMET framework, one can start from a static "gluon spin" operator, which is defined as $\vec{E} \times \vec{A}$ fixed in a time-independent gauge which maintains the transverse polarizations of the gluon field in the IMF limit. For example, the Coulomb gauge $\vec{\nabla} \cdot \vec{A}=0$, axial gauges $A^{z}=0$ and $A^{0}=0$ are viable options (Hatta et al., 2014).

In the Coulomb gauge and $\overline{\mathrm{MS}}$ scheme, the static "gluon spin" $\Delta \widetilde{G}$ in a massive on-shell quark state at oneloop order is (Chen et al., 2011; Ji et al., 2013b)

$$
\begin{aligned}
\Delta \widetilde{G}\left(P^{z}, \mu\right) & \left(2 S^{z}\right)=\left.\left\langle P S\left|\epsilon_{\perp}^{i j} F^{i 0} A^{j}\right| P S\right\rangle_{q}\right|_{\vec{\nabla} \cdot \vec{A}=0} \\
= & \frac{\alpha_{s} C_{F}}{4 \pi}\left[\frac{5}{3} \ln \frac{\mu^{2}}{m^{2}}-\frac{1}{9}+\frac{4}{3} \ln \frac{\left(2 P^{z}\right)^{2}}{m^{2}}\right]\left(2 S^{z}\right),
\end{aligned}
$$

where the subscript $q$ denotes a quark, and $S^{\mu}$ is the spin vector. The collinear divergence is regulated by the finite quark mass $m$.

If we follow the procedure in (Weinberg, 1966) and take $P^{z} \rightarrow \infty$ limit before UV regularization (Ji et al. , 2013b), then

$$
\begin{aligned}
\Delta \widetilde{G}(\infty, \mu)\left(2 S^{z}\right) & =\left.\left\langle P S\left|\epsilon_{\perp}^{i j} F^{i 0} A^{j}\right| P S\right\rangle_{q}\right|_{\vec{\nabla} \cdot \vec{A}=0} \\
& =\frac{\alpha_{s} C_{F}}{4 \pi}\left(3 \ln \frac{\mu^{2}}{m^{2}}+7\right)\left(2 S^{z}\right),
\end{aligned}
$$


which is exactly the same as the light-cone gluon helicity $\Delta G(\mu)$ (Hoodbhoy et al., 1999a). Therefore, despite the difference in the UV divergence, the collinear divergences of $\Delta \widetilde{G}\left(P^{z}, \mu\right)$ and $\Delta G(\mu)$ are exactly the same, which allows for a perturbative matching between them.

The complete factorization formula that relates $\Delta \widetilde{G}\left(P^{z}, \mu\right)$ to $\Delta G$ and $\Delta \Sigma$ is

$$
\begin{aligned}
\Delta \widetilde{G}\left(P^{z}, \mu\right)=Z_{g g}( & \left.P^{z} / \mu\right) \Delta G(\mu) \\
& +Z_{g q}\left(P^{z} / \mu\right) \Delta \Sigma(\mu)+\ldots
\end{aligned}
$$

where $\ldots$ are power corrections suppressed by $1 / P^{z}$, and the matching coefficients $Z_{g g}$ and $Z_{g g}$ have been calculated for the Coulomb gauge at one-loop (Ji et al., 2015d).

Besides, one can also calculate the gluon helicity PDF $\Delta g(x)$ according to the factorization formula in Sec. III, and then integrate it over $x$ to obtain $\Delta G$.

At leading-twist, apart from the unpolarized and helicity PDFs that we have discussed before, there is also the transversity PDF defined as (Jaffe and Ji, 1991, 1992)

$h_{1}(x)=\frac{1}{2 P^{+}} \int \frac{d \lambda}{2 \pi} e^{-i \lambda x}\left\langle P S_{\perp}\left|\bar{\psi}(0) \gamma^{+} \gamma_{\perp} \gamma_{5} \psi(\lambda n)\right| P S_{\perp}\right\rangle$

The $h_{1}(x)$ simply counts the number of transversely polarized quarks carrying the momentum fraction $x$ in a transversely polarized proton. The first moment of this distribution corresponds to the socalled tensor charge $\delta q$, which is the matrix element of a chiral-odd operator. $h_{1}(x)$ can be accessed through the transverse-transverse spin asymmetry in Drell-Yan processes (Jaffe and Ji, 1991, 1992; Ralston and Soper, 1979) or the Collins single-spin asymmetry in SIDIS where the transversity TMDPDF couples to a chiral-odd TMD fragmentation function Collins, 1993). At present, experimental results on the transversity PDF are very limited (Barone et al., 2002; Cammarota et al., 2020; Kang et al., 2016; Lin et al., 2018b; Radici and Bacchetta, 2018), especially for the sea quark contributions (Chang and Peng, 2014), so this is one scenario where lattice QCD calculation can make an important difference.

The LaMET calculation of $h_{1}(x)$ is straightforward as the nonlocal operator has the same renormalization as the unpolarized case, and its one-loop matching has been calculated in the $\overline{\mathrm{MS}}$ and $\mathrm{RI} / \mathrm{MOM}$ schemes at one-loop order (Alexandrou et al., 2018b; Liu et al., 2018). First lattice calculations of $h_{1}(x)$ have been done in Alexandrou et al., 2018b; Chen et al., 2016; Liu et al., 2018), which will be discussed with more details in Sec. VI.

\section{GENERALIZED COLLINEAR PARTON OBSERVABLES}

In the previous section, we have extensively discussed the leading-twist collinear PDFs that characterize the 1D structure of the proton in longitudinal momentum space. There exist various other parton observables that provide complementary information. In this section, we focus on observables defined by collinear parton correlators, in the sense that only the collinear quark and gluon mode contribute, corresponding to the so-called collinear expansion in QCD factorizations (Collins, 2011a; Sterman, 1993). We call them "generalized collinear parton observables" (GCPOs), and discuss their calculations through LaMET framework. For observables defined by parton correlators involving transverse separations, in particular, the TMDPDFs, Wigner functions, and LFWFs, we will consider them in the following sections.

One of the important GCPOs is the GPDs introduced in (Müller et al., 1994), and rediscovered (Ji, 1997b) from their connection to the spin structure of the proton. They describe the correlation between the transverse position and longitudinal momentum of partons inside the proton, and thus provide important information for 3D imaging of the proton. A proton spin sum rule was derived in terms of the moments of the GPDs, which has stimulated considerable general interest in the GPDs. It was also found that in the so-called zero skewness limit or when the longitudinal momentum transfer vanishes, the GPD has a probability interpretation in the impact parameter space (Burkardt, 2000). In general case, it is related to the quantum phase-space distributions or Wigner functions (Belitsky et al., 2004; Ji, 2003). Experimentally, the GPDs can be measured through hard exclusive processes such as deeply virtual Compton scattering (DVCS) or meson production (DVMP) that were first proposed in (Ji, 1997a, b). Much effort has been devoted to measuring such processes at completed and ongoing experiments, including HERA, COMPASS and JLab. For a more comprehensive discussion on the GPDs, we refer the readers to the review articles (Belitsky and Radyushkin, 2005; Diehl, 2003; Ji, 2004, 1998). Despite that the GPDs have more complicated kinematic dependence and relation to experimental observables, various fitting methods have been proposed in the literature to fit available DVCS and DVMP data (Favart et al., 2016; Kumericki et al., 2016). In parallel, one can also extract certain information on the GPDs from lattice calculations of their moments (Alexandrou et al., 2020b; Gockeler et al., 2004; Hagler et al., 2008), which, however, is again very limited due to the same difficulties existing in lattice calculations of the PDF moments. For JLab $12 \mathrm{GeV}$ program and future EIC, it is critically important to have first-principle calculations of GPDs with much better understanding of the physical landscape in different kinematic variables.

A simpler but closely related GCPO is the parton distribution amplitudes (DAs), which are collinear ma- 
trix elements of light-cone operators between a hadron state and the QCD vacuum, representing the probability amplitude of finding a given Fock state in the hadron. They can be probed in certain exclusive processes (Brodsky, 2002), and are crucial inputs for processes relevant to measuring fundamental parameters of the Standard Model and probing new physics. There exists a vast amount of literature on this subject, particularly about the pion DA. For a review see e.g. (Braun, 2006; Brodsky and Lepage, 1989; Grozin, 2005).

Another type of GCPO is the higher-twist parton distributions. They are defined by multi-parton correlation functions, and quantify the proton structure in terms of longitudinal momentum correlations (Ellis et al., 1983; Jaffe and Ji, 1992; Jaffe and Soldate, 1982). Although physically interesting, they are hard to separate theoretically due to mixing with the leading-twist ones (Ji), 1995; Mueller, 1985), and difficult to extract experimentally because they are power-suppressed (Ji, 1993). Higher-twist effects can become important in kinematic regions where the suppression is relaxed. Moreover, some twist-three distributions, $g_{T}$ and $h_{L}$, are different; they have no leading-twist to mix with and are dominant in spin-related observables (Jaffe and Ji, 1992). Twist-three GPDs are also relevant for studying parton OAM in the proton (Courtoy et al., 2014; Hatta and Yoshida, 2012; Ji et al., 2013a) and can be accessed through DVCS process (Kiptily and Polyakov, 2004; Penttinen et al., 2000).

In principle, all the GCPOs discussed above can be computed within LaMET. In addition, an accurate LaMET expansion for the leading-twist PDFs requires calculations of quasi higher-twist matrix elements. In the following, we begin with the flavor non-singlet quark GPDs and hadronic DAs for which the computational procedure has been well established, and then give some generic discussions on higher-twist distributions, followed by the discussion on power-suppressed contributions required to extract the leading-twist quark PDFs, which have been investigated using different approaches though not yet implemented in numerical computations.

\section{A. Generalized Parton Distributions}

The operators defining the GPDs are the same as those defining the PDFs. Thus, the LaMET calculation of PDFs can be rather straightforwardly generalized to the GPDs by taking into account the non-forward kinematics (Liu et al., 2019b). To illustrate how it works, let us take the nonsinglet unpolarized quark GPDs in the nucleon as an example.

The unpolarized quark GPDs are defined through the following matrix element ( $\mathrm{Ji}$, 2004)

$$
\begin{aligned}
F & =\frac{1}{2 \bar{P}^{+}} \int \frac{d \lambda}{2 \pi} e^{-i x \lambda}\left\langle P^{\prime} S^{\prime}\left|O_{\gamma^{+}}(\lambda n)\right| P S\right\rangle \\
& =\frac{1}{2 \bar{P}^{+}} \bar{u}\left(P^{\prime} S^{\prime}\right)\left[H \gamma^{+}+E \frac{i \sigma^{+\mu} \Delta_{\mu}}{2 M}\right] u(P S),
\end{aligned}
$$

where we have suppressed the arguments $(x, \xi, t, \mu)$ of $F$, $H$ and $E$ for simplicity. The operator

$$
O_{\gamma^{+}}(\lambda n)=\bar{\psi}\left(\frac{\lambda n}{2}\right) \gamma^{+} W\left(\frac{\lambda n}{2},-\frac{\lambda n}{2}\right) \psi\left(-\frac{\lambda n}{2}\right)
$$

with $n^{\mu}=1 / \sqrt{2}\left(1 / \bar{P}^{+}, 0,0,-1 / \bar{P}^{+}\right)$is the same operator used to define the unpolarized quark PDF, $M$ is the nucleon mass. The momentum fraction $x \in[-1,1]$, and

$$
\Delta \equiv P^{\prime}-P, \quad t \equiv \Delta^{2}, \quad \xi \equiv-\frac{P^{\prime+}-P^{+}}{P^{\prime+}+P^{+}}=-\frac{\Delta^{+}}{2 \bar{P}^{+}},
$$

where without loss of generality we have chosen a Lorentz frame in which the average momentum takes the following form

$$
\bar{P}^{\mu} \equiv \frac{P^{\prime \mu}+P^{\mu}}{2}=\left(\bar{P}^{0}, 0,0, \bar{P}^{z}\right)
$$

The skewness parameter $\xi \in[-1,1]$ since $P^{+}, P^{\prime+} \geq 0$. Besides, there exists another kinematic constraint on $\xi$, which follows from $\vec{\Delta}_{\perp}^{2} \geq 0$,

$$
\xi \leq \xi_{\max }(t)=\sqrt{\frac{-t}{-t+4 M^{2}}} .
$$

In the following, we will also assume $\xi>0$ without loss of generality. With these kinematic constraints, the GPDs can be divided into several kinematic regions that have different physical interpretations. As shown in Fig. 9, in the region $\xi<x<1(-1<x<-\xi)$ the distribution describes the emission and reabsorption of a quark (antiquark), while in the region $-\xi<x<\xi$ it represents the creation of a quark and antiquark pair. The first region is similar to that present in usual PDFs and referred to as the DGLAP region, whereas the second is similar to that in a meson DA, which will be discussed later in this section, and referred to as the Efremov-RadyushkinBrodsky-Lepage (ERBL) region. The easiest way to see this is in light-cone quantization and light-cone gauge where the matrix element defining the GPDs can be rewritten in terms of parton creation and annihilation operators, for details see e.g. (Ji, 2004).

The quark GPDs defined above have a number of remarkable properties, see, e.g., Belitsky and Radyushkin, 2005; Diehl, 2003; Ji, 2004, 1998), which either hold or have similar counterparts for the quark quasi-GPDs to be defined below. Apart from their physical significance, these properties also serve as useful checks on calculations related to GPDs. 

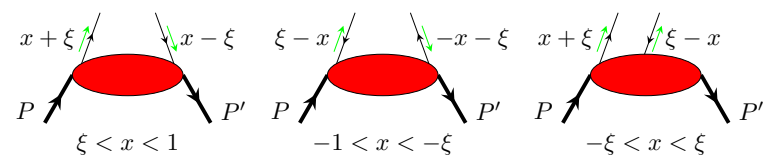

FIG. 9: Parton interpretation of the GPDs in different kinematic regions.

According to LaMET, the unpolarized quark GPDs defined above can be determined by calculating the following quasi-GPDs

$$
\begin{aligned}
\tilde{F} & =\frac{1}{2 \bar{P}^{0}} \int \frac{d \lambda}{2 \pi} e^{i y \lambda}\left\langle P^{\prime} S^{\prime}\left|O_{\gamma^{0}}(z)\right| P S\right\rangle \\
& =\frac{1}{2 \bar{P}^{0}} \bar{u}\left(P^{\prime} S^{\prime}\right)\left\{\tilde{H} \gamma^{0}+\tilde{E} \frac{i \sigma^{0 \mu} \Delta_{\mu}}{2 M}\right\} u(P S),
\end{aligned}
$$

where we have again suppressed the arguments $\left(y, \tilde{\xi}, t, \bar{P}^{z}, \mu\right)$ of $\tilde{F}, \tilde{H}$, and $\tilde{E}$. The operator $O_{\gamma^{0}}(z)=$ $\bar{\psi}\left(\frac{z}{2}\right) \gamma^{0} W\left(\frac{z}{2},-\frac{z}{2}\right) \psi\left(-\frac{z}{2}\right)$ is the same operator defining the unpolarized quark quasi-PDF, and $\lambda=z \bar{P}^{z}$. As in the quasi-PDF case, the momentum fraction $y$ extends from $-\infty$ to $\infty$. The skewness parameter for the quasi-GPD

$$
\tilde{\xi}=-\frac{P^{\prime z}-P^{z}}{P^{\prime z}+P^{z}}=-\frac{\Delta^{z}}{2 \bar{P}^{z}}=\xi+\mathcal{O}\left(\frac{M^{2}}{\left(\bar{P}^{z}\right)^{2}}, \frac{t}{\left(\bar{P}^{z}\right)^{2}}\right)
$$

differs from the light-cone skewness $\xi$ by power suppressed corrections. Moreover, the constraint from $\vec{\Delta}_{\perp}^{2} \geq$ 0 becomes (Ji et al., 2015a)

$$
\tilde{\xi} \leq \frac{1}{2 \bar{P}^{z}} \sqrt{\frac{-t\left[\left(\bar{P}^{z}\right)^{2}+M^{2}-t / 4\right]}{M^{2}-t / 4}},
$$

which differs from the constraint in Eq. (132) by corrections of $\mathcal{O}\left(M^{2} /\left(\bar{P}^{z}\right)^{2}, t /\left(\bar{P}^{z}\right)^{2}\right)$. We can replace $\tilde{\xi}$ with $\xi$ and attribute the difference to generic power suppressed contributions.

The quasi-GPDs defined above can be renormalized by observing that their UV divergence depends only on the operators defining them, but not on the external states. Since $O_{\gamma^{0}}(z)$ is multiplicatively renormalized, we can choose the same renormalization factor as that for the quasi-PDF (Liu et al., 2020; Stewart and Zhao, 2018) to renormalize the quasi-GPD. After renormalization, the quasi-GPD can then be matched to the usual GPD through a factorization formula.

The factorization of quasi-GPDs was first proposed and verified at one-loop order in (Ji et al., 2015a; Xiong and Zhang, 2015), where a transverse momentum cutoff and a quark mass were used as the UV and IR regulator, respectively. Later on, a detailed derivation based on OPE was given in (Liu et al., 2019a). In contrast with the OPE for the quasi-PDF, a crucial difference here is that the total derivative of operators can come into play, as it simply gives momentum transfer factors when sandwiched between non-forward external states, and therefore is non-vanishing. In other words, the local twist-two operators as those in Eq. (97) will mix under renormalization with operators with total derivatives. The RGE that governs the mixing reads (Braun et al., 2003),

$$
\begin{aligned}
& \mu^{2} \frac{d}{d \mu^{2}} O^{\mu_{0} \mu_{1} \ldots \mu_{n}}(\mu)=\sum_{m=0}^{[n / 2]} \Gamma_{n m} \\
& \times\left[i \partial^{\left(\mu_{1}\right.} \cdots i \partial^{\mu_{2 m}} \bar{\psi} \gamma^{\mu_{0}} i \overleftrightarrow{D^{\mu_{2 m+1}}} \cdots i \overleftrightarrow{D}^{\left.\mu_{n}\right)} \psi-\text { trace }\right]
\end{aligned}
$$

where $\Gamma_{n m}$ is the anomalous dimension of the associated operators, $\overleftrightarrow{D}=(\vec{D}-\overleftarrow{D}) / 2$ with $\vec{D}(\overleftarrow{D})$ denoting the covariant derivative acting to the right (left). The above equation can be diagonalized by choosing an appropriate operator basis. Such an operator basis has been studied in the literature and known as the "renormalization group improved" conformal operators Braun et al., 2003; Mueller, 1994b). In terms of the matrix elements of these operators, we have

$$
\begin{array}{r}
\left\langle P^{\prime}\left|O_{\gamma^{0}}(z)\right| P\right\rangle=2 P^{0} \sum_{n=0}^{\infty} C_{n}\left(\mu^{2} z^{2}\right) \mathcal{F}_{n}(-\lambda) \sum_{m=0}^{n} \mathcal{B}_{n m}(\mu) \\
\times \xi^{n} \int_{-1}^{1} d x C_{m}^{3 / 2}\left(\frac{x}{\xi}\right) F(x, \xi, t, \mu)+\ldots, \quad(137)
\end{array}
$$

where $\mathcal{F}_{n}(-\lambda)$ are partial wave polynomials whose explicit forms are known in the conformal OPE of current-current correlators for the hadronic lightcone DAs (Braun and Müller, 2008), $\mathcal{B}_{n m}$ can be found in (Braun et al., 2003; Mueller, 1994b), and $\ldots$ denotes the higher-twist contributions $\mathcal{O}\left(M^{2} /\left(\bar{P}^{z}\right)^{2}, t /\left(\bar{P}^{z}\right)^{2}, z^{2} \Lambda_{\mathrm{QCD}}^{2}\right)$.

Fourier transforming the l.h.s of the above equation to momentum space and invert it order by order in $\alpha_{s}$, we then obtain the following EFT expansion of the unpolarized quark GPD,

$$
\begin{aligned}
F & (x, \xi, t, \mu) \\
& =\int_{-\infty}^{\infty} \frac{d y}{|\xi|} \bar{C}\left(\frac{x}{\xi}, \frac{y}{\xi}, \frac{\mu}{\xi \bar{P}^{z}}\right) \tilde{F}\left(y, \xi, t, \bar{P}^{z}, \mu\right)+\ldots \\
& =\int_{-\infty}^{\infty} \frac{d y}{|y|} C\left(\frac{x}{y}, \frac{\xi}{y}, \frac{\mu}{y \bar{P}^{z}}\right) \tilde{F}\left(y, \xi, t, \bar{P}^{z}, \mu\right)+\ldots
\end{aligned}
$$

which has been organized following the same spirit as the factorization of PDFs in previous sections. Both forms have been used in the literature (Ji et al., 2015a; Liu et al., 2019a; Xiong and Zhang, 2015) with the matching coefficients being related by

$$
C\left(\frac{x}{y}, \frac{\xi}{y}, \frac{\mu}{y \bar{P}^{z}}\right)=\left|\frac{y}{\xi}\right| \bar{C}\left(\frac{x}{\xi}, \frac{y}{\xi}, \frac{\mu}{\xi \bar{P}^{z}}\right),
$$

and ... denotes the higher-twist contributions which have the same power-counting as in Eq. (137) except that $z^{2}$ 
is replaced by $1 /\left(x \bar{P}^{z}\right)^{2}$. For the helicity and transversity quark quasi-GPDs, the factorization formula has the same form as Eq. (138) (Liu et al., 2019a).

The matching coefficient can be obtained by replacing the hadron states in Eqs. (128) and (133) with the quark states carrying momentum $p+\Delta / 2$ and $p-\Delta / 2$ with $p^{\mu}=\left(p^{0}, 0,0, p^{z}\right)$, and calculating the quark matrix element in perturbation theory. The explicit expression for the $\mathcal{O}\left(\alpha_{s}\right)$ matching coefficients can be found in (Liu et al., 2019a). An important feature of the result is: The quasi-GPDs do not vanish in all $y$ range, but the collinear singularities only show up in DGLAP and ERBL regions at one-loop. They are exactly the same as those in light-cone GPDs, and thus cancel in the matching coefficient. Moreover, one can derive momentum RGEs for the quasi-GPDs, which are turned into RGE for the scale dependence of the GPDs by the matching procedure.

To conclude this subsection, let us make some remarks on the EFT formula for the quark GPD above. First, at zero skewness $\xi=0$, we have

$$
\begin{gathered}
F(x, 0, t, \mu)=\int_{-\infty}^{\infty} \frac{d y}{|y|} C\left(\frac{x}{y}, 0, \frac{\mu}{y P^{z}}\right) \tilde{F}\left(y, 0, t, P^{z}, \mu\right) \\
+\ldots,
\end{gathered}
$$

where the matching kernel $C\left(x / y, 0, \mu /\left(y P^{z}\right)\right)$ is exactly the same as the matching coefficient for the quasiPDF (Izubuchi et al., 2018), even when $t \neq 0$. This can be understood as follows: At zero skewness, both the longitudinal momentum transfer and the energy transfer vanish, the momentum transfer is purely transverse and thus is not affected by Lorentz boost along the longitudinal $z$ direction. As a result, no extra matching related to $t$ is required in the large $P^{z}$ limit, and the matching remains the same as in the quasi-PDF case. If we take the forward limit $\Delta \rightarrow 0$, then Eq. (140) reduces exactly to the EFT expansion formula for the PDF (Izubuchi et al., 2018; Ji et al., 2015a).

Second, in the limit $\xi \rightarrow 1$ and $t \rightarrow 0$, the quasi-GPD reduces to the quasi-DA that will be discussed in the next subsection, and the corresponding matching kernel also reduces to that for the quasi-DA.

\section{B. Hadronic Distribution Amplitudes}

Within LaMET, the DAs of protons as well as other hadrons can also be extracted from lattice simulations of appropriately chosen quasi-DAs. In this subsection, we show how this can be done in practice. For illustration, we take the leading-twist pion DA as an example. The application to other hadrons (Wang et al., 2019a; Zhang et al., 2019c) is analogous.

The leading-twist DA of the pion is the simplest and most extensively studied hadronic DA. It represents the probability amplitude of finding the valence $q \bar{q}$ Fock state in the pion with the quark carrying a fraction $x$ of the total pion momentum, and is defined as

$$
\phi_{\pi}(x)=\frac{1}{i f_{\pi}} \int \frac{d \lambda}{2 \pi P^{+}} e^{-i\left(x-\frac{1}{2}\right) \lambda}\left\langle 0\left|O_{\gamma^{+} \gamma_{5}}(\lambda n)\right| \pi(P)\right\rangle,
$$

with normalization $\int_{0}^{1} d x \phi_{\pi}(x)=1$. Here $f_{\pi}$ denotes the decay constant, and $O_{\gamma^{+} \gamma_{5}}(\lambda n)$ has the same structure as that used in Eq. (128) with $\gamma^{+}$replaced by $\gamma^{+} \gamma_{5}$. The pion DA can be constrained from experimental measurements of, e.g., $\gamma \gamma * \rightarrow \pi^{0}$ from BaBar and Belle (Aubert et al., 2009; Uehara et al., 2012), and then used as an input to test QCD in other measurements such as the pion form factor (Efremov and Radvushkin, 1980; Farrar and Jackson, 1979). In the asymptotic limit, it is well known that the pion DA takes the form $6 x(1-x)$ (Efremov and Radyushkin, 1980; Lepage and Brodsky, 1979). However, how it behaves at lower scales remains under debate (see e.g. (Chernyak and Zhitnitsky, 1982)). Calculating the pion DA with controllable systematics in LaMET will be able to shed new lights on its shape and thus on our understanding of pion structure.

Following the same strategy as before, we can access the $x$-dependence of the pion DA by studying the following quasi-DA (Ji et al., 2015a; Zhang et al., 2017)

$$
\tilde{\phi}_{\pi}\left(y, P^{z}\right)=\frac{1}{i f_{\pi}} \int \frac{d \lambda}{2 \pi P^{z}} e^{i\left(y-\frac{1}{2}\right) \lambda}\left\langle 0\left|O_{\gamma^{z} \gamma_{5}}(z)\right| \pi(P)\right\rangle,
$$

The longitudinally and transversely polarized vector meson quasi-DAs can be defined analogously by replacing $\gamma^{z} \gamma_{5}$ in the above equation with $\gamma^{0}, \gamma^{z} \gamma_{\perp}$, respectively (Liu et al., 2019b).

The quark bilinear operators defining quasi-DAs follow the same renormalization pattern as those defining the quasi-PDFs or quasi-GPDs. In the literature, the Wilson-line mass-subtraction scheme was used in the first LaMET calculations of the meson DAs (Zhang et al., 2017, 2019c), and the RI/MOM scheme has been adopted in more recent works (Zhang et al., 2020b).

The LaMET expression for DAs takes the following form in the $\overline{\mathrm{MS}}$ scheme (Ji et al., 2015a; Liu et al., 2019b)

$$
\phi_{\pi}(x, \mu)=\int_{-\infty}^{\infty} d y C_{\pi}\left(x, y, P^{z} / \mu\right) \tilde{\phi}_{\pi}\left(y, P^{z}, \mu\right)+\ldots
$$

The matching coefficient for the quasi-DAs can be obtained by replacing the meson state $|\pi(P)\rangle$ in Eqs. (141) and (142) with the lowest Fock state $\mid q(y P) \bar{q}((1-$ $y) P)\rangle$ and calculating the quark matrix elements, where $y P$ and $(1-y) P$ are the momenta of the quark $q$ and anti-quark $\bar{q}$, respectively. Its one-loop results have been calculated in both $\overline{\mathrm{MS}}$ and $\mathrm{RI} / \mathrm{MOM}$ 
schemes (Liu et al., 2019b), which agrees with matching coefficient for the quasi-GPDs (Ji et al., 2015a; Liu et al., 2019a; Xiong and Zhang, 2015) in Eq. (138) with the replacement of $\xi \rightarrow 1 /(2 y-1), x / \xi \rightarrow 2 x-1$, and the external momentum $p^{z}$ to $p^{z} / 2$.

Apart from the LaMET approach in momentum space, the shape of the pion DA has also been studied using equal-time current-current correlation in coordinate space approach (Bali et al., 2018a, b) ,

$$
\begin{aligned}
\left\langle 0\left|T\left\{J_{\mu}\left(\frac{z}{2}\right) J_{\nu}\left(-\frac{z}{2}\right)\right\}\right| \pi^{0}(P)\right\rangle & \\
= & \frac{2 i f_{\pi}}{3 \pi^{2} z^{4}} \epsilon_{\mu \nu \alpha \beta} P^{\alpha} z^{\beta} \Phi_{\pi}\left(\lambda, z^{2}\right),
\end{aligned}
$$

where $\Phi_{\pi}\left(\lambda, z^{2}\right)$ can be factorized as

$$
\Phi_{\pi}\left(\lambda, z^{2}\right)=C_{2}\left(\lambda, z^{2} \mu^{2}, x\right) \otimes \phi_{\pi}(x, \mu)+\cdots .
$$

Here the matching coefficient $C_{2}$ depends on the choice of the currents. Its explicit expression can be found in (Bali et al., 2018a). The above factorization is controlled by $\mathcal{O}\left(z^{2} \Lambda_{\text {OCD }}^{2}\right)$, with power corrections denoted by "...". In (Bali et al., 2018a), a combined analysis of several current-current correlations has been performed where twist-four contributions were also included using the model estimate in (Ball et al., 2006; Braun and Filvanov, 1990). The leading-twist pion DA was then extracted from a global fit to the data, and the second moment of the pion DA has been fitted with controlled precision, both of which favor a considerably broader shape than the asymptotic DA at a scale of 2 $\mathrm{GeV}$. A large pion momentum is required to access information at large $\lambda$ so that we can extract wider range of $x$ or higher moments of the pion DA (Bali et al., 2018b).

\section{Higher-Twist Distributions}

Higher-twist distributions are quantities of great interest because they describe the coherent quark-gluon correlations in the proton. In contrast with the leading-twist distributions, our understanding of the higher-twist ones is rather poor. On one hand, they often depend on more than one parton momentum fractions; on the other hand, there is no physical intuition about what they may look like, in particular, about how they behave asymptotically at small and large $x$ (Braun et al., 2011). There have been studies on the higher-twist distributions in the context of their connection to the DIS structure function, the transverse single-spin asymmetries in various hadron productions, GPDs related to quark and gluon OAM, parton DAs, etc. LaMET will be able to shed new lights by providing a possibility to access them from the lattice.

Higher-twist contributions also appear in LaMET expansion, where the suppression is provided by powers of the hadron momentum squared. In all factorizations presented in previous sections, only the leading-twist terms that capture the logarithmic dependence on hadron momentum are taken into account. The higher-twist contributions have been assumed to be small. If the hadron momentum is not sufficiently large compared and/or one is close to the endpoint region $(x \rightarrow 0$ and $x \rightarrow 1)$, the higher-twist contributions can become non-negligible, whose structure and impact require understanding.

\section{Higher-twist collinear-parton observables}

Beyond leading-twist, there exist three simplest twistthree quark distributions $e(x), g_{T}(x)$ and $h_{L}(x)$ related to the unpolarized, transversely and longitudinally polarized proton (Jaffe and Ji, 1992),

$$
\begin{aligned}
e(x)= & \frac{1}{2 M} \int \frac{d \lambda}{2 \pi} e^{i x \lambda} \\
& \times\left\langle P S\left|\psi_{+}^{\dagger}(0) \gamma_{0} \psi_{-}(\lambda n)\right| P S\right\rangle+\text { h.c. } \\
g_{T}(x)= & \frac{1}{2 M} \int \frac{d \lambda}{2 \pi} e^{i x \lambda} \\
& \times\left\langle P S_{\perp}\left|\psi_{+}^{\dagger}(0) \gamma_{0} \gamma_{\perp} \gamma_{5} \psi_{-}(\lambda n)\right| P S_{\perp}\right\rangle+\text { h.c. } \\
h_{L}(x)= & \frac{1}{2 M} \int \frac{d \lambda}{2 \pi} e^{i x \lambda} \\
& \times\left\langle P S_{z}\left|\psi_{+}^{\dagger}(0) \gamma_{0} \gamma_{5} \psi_{-}(\lambda n)\right| P S_{z}\right\rangle+\text { h.c. }
\end{aligned}
$$

where we have again employed the decomposition of quark fields $\psi=\psi_{+}+\psi_{-}$in Sec. I.A and the light-cone gauge $A^{+}=0$, and "h.c." stands for Hermitian conjugate.

The twist-three distributions can contribute as leading effects in certain experimental observables. For example, $g_{T}(x)$ and $h_{L}(x)$ can be measured as the leading effects in the longitudinal-transverse spin asymmetry in polarized Drell-Yan process.

Since $\psi_{-}$is a non-dynamical component depending on $\psi_{+}$, all the above distributions can be shown to be related to more complicated quark-gluon correlation functions (Balitsky et al., 1996; Ji and Chou, 1990). A complete set of such correlation functions has been given in (Ji, 1992; Ji and Osborne, 2001; Kang and Qiu, 2009; Qiu and Sterman, 1991), where the quark-gluon correlations in a transversely-polarized proton take the following form

$$
\begin{gathered}
T_{q}\left(x_{1}, x_{2}\right)=\frac{1}{\left(P^{+}\right)^{2}} \int \frac{d \lambda d \zeta}{(2 \pi)^{2}} e^{i \lambda x_{1}+i \zeta\left(x_{2}-x_{1}\right)} \\
\times\left\langle P S_{\perp}\left|\bar{\psi}(0) \gamma^{+} \epsilon^{+-S_{\perp} i} g F^{+i}(\zeta n) \psi(\lambda n)\right| P S_{\perp}\right\rangle \\
T_{\Delta q}\left(x_{1}, x_{2}\right)=\frac{1}{\left(P^{+}\right)^{2}} \int \frac{d \lambda d \zeta}{(2 \pi)^{2}} e^{i \lambda x_{1}+i \zeta\left(x_{2}-x_{1}\right)} \\
\times\left\langle P S_{\perp}\left|\bar{\psi}(0) i \gamma^{+} \gamma_{5} S_{\perp}^{i} g F^{+i}(\zeta n) \psi(\lambda n)\right| P S_{\perp}\right\rangle
\end{gathered}
$$

There are also ones in an unpolarized and longitudinallypolarized proton. Generalizing to off-forward kinematics, the resulting twist-three GPDs are also related 
to quark and gluon OAM contribution to the proton spin (Hatta and Yoshida, 2012; Ji et al., 2013a).

One can also define twist-four distributions in a similar way as in Eq. (149) by using $\psi_{-}$for both quark fields. More general twist-four distributions will involve three light-cone variables, which will contribute to, e.g., $1 / Q^{2}$ term in DIS (Ellis et al., 1983; Jaffe and Ji, 1992; Jaffe and Soldate, 1982; Ji, 1993).

In principle, all the above higher-twist distributions, as well as others that have not been listed here, can be computed using the LaMET approach by choosing appropriate quasi-LF correlations. For example, the first exploratory lattice calculation of $g_{T}(x)$ has been done in (Bhattacharva et al., 2020a), which will be discussed in Sec. VI.C However, extra complications are expected due to their complex structure. For instance, the lightcone zero modes that do not enter in dealing with leadingtwist distributions come into play here. Recently, one of the authors has shown how to study the properties of these zero modes from lattice simulations in LaMET (Ji), 2020). In addition, the higher-twist distributions will have a more complex mixing pattern (Balitsky et al., 1996; Ji and Chou, 1990). Thus, their matching from the corresponding quasi distributions must take into account such mixings, making them more challenging than calculating the twist-two PDFs. One-loop studies of the matching for twist-three disributions have been carried out in (Bhattacharya et al., 2020b, c).

\section{Higher-twist contributions to quasi-PDFs}

Let us turn to the power suppressed higher-twist contributions appearing in the extraction of leading-twist quark PDFs using LaMET. Such contributions have two distinct origins. To understand them, let us recall the OPE for the quasi-LF correlation in Eq. (98). For simplicity, we ignore the renormalization here. Recovering the leading-twist quark PDF requires removing the contributions of both trace terms in that equation. The trace terms on the r.h.s. of Eq. (98), which lead to contributions suppressed by powers of $M^{2} /\left(P^{z}\right)^{2}$, are known as kinematic power contributions or target mass corrections. In DIS, they can be accounted for by changing the scaling variable $x$ to the Nachtmann variable (Nachtmann, 1973). In the case of LaMET, it behaves slightly differently, as shown in the following. The second type of power corrections come from the trace terms in the operators on the r.h.s. of Eq. (97), and in general leads to contributions of $\mathcal{O}\left(\Lambda_{\mathrm{QCD}}^{2} /\left(P^{z}\right)^{2}\right)$. These are genuine higher-twist contributions that involve multi-parton correlations, sometimes also known as dynamical highertwist contributions. The target mass corrections have been computed to all orders in $M^{2} /\left(P^{z}\right)^{2}$ for the quark quasi-PDFs in (Chen et al., 2016; Radyushkin, 2017c). The genuine higher-twist contributions have been investi- gated using two different approaches (Braun et al., 2019; Chen et al., 2016).

According to (Chen et al., 2016), the $M^{2} /\left(P^{z}\right)^{2}$ corrections can be obtained from the ratio

$$
K_{m} \equiv \frac{n_{\left(\mu_{1}\right.} \cdots n_{\left.\mu_{m}\right)} P^{\mu_{1}} \cdots P^{\mu_{m}}}{n_{\mu_{1}} \cdots n_{\mu_{m}} P^{\mu_{1}} \cdots P^{\mu_{m}}}=\sum_{i=0}^{i_{\max }} C_{m-i}^{i} c^{i},
$$

where $i_{\max }=(m-\operatorname{Mod}[m, 2]) / 2, C$ is the binomial function and $c=-n^{2} M^{2} / 4(n \cdot P)^{2}=M^{2} / 4\left(P^{z}\right)^{2}$ with $n^{\mu}=(0,0,0,-1)$ and $n \cdot P=P^{z}$.

Plugged into the tree-level OPE formula in Eq. (98), the above factors can then be converted to the following relation between unpolarized PDF and quasiPDF (Chen et al., 2016)

$$
\begin{gathered}
q(x)=\sqrt{1+4 c} \sum_{n=0}^{\infty} \frac{(4 c)^{n}}{f_{+}^{2 n+1}}\left[\left(1+(-1)^{n}\right) \tilde{q}\left(\frac{f_{+}^{2 n+1} x}{2(4 c)^{n}}\right)\right. \\
\left.+\left(1-(-1)^{n}\right) \tilde{q}\left(\frac{-f_{+}^{2 n+1} x}{2(4 c)^{n}}\right)\right],
\end{gathered}
$$

where $f_{+}=\sqrt{1+4 c}+1$. It is worth noting that quark number conservation is preserved in the above result. The target mass corrections for the longitudinally and transversely polarized quasi-PDFs can be derived analogously.

The trace part on the r.h.s. of Eq. (97) is a genuine higher-twist effect. One may try to construct a non-local form of the higher-twist operators from OPE. The leading trace term, which is a twist-four effect, has been studied in (Chen et al., 2016) (see also Balitsky and Braun, 1989)) and shown to give rise to a twist-four PDF

$$
q_{4}\left(x, P^{z}\right)=\int_{-\infty}^{\infty} \frac{d \lambda}{8 \pi P^{z}} \Gamma_{0}(-i x \lambda)\left\langle P\left|O_{\mathrm{tr}}(z)\right| P\right\rangle,
$$

with

$$
\begin{aligned}
& O_{\operatorname{tr}}(z)=\int_{0}^{z} d z_{1} \bar{\psi}(0)\left[\Gamma^{\nu} W\left(0, z_{1}\right) D_{\nu} W\left(z_{1}, z\right)\right. \\
& \left.+\int_{0}^{z_{1}} d z_{2} n \cdot \Gamma W\left(0, z_{2}\right) D^{\nu} W\left(z_{2}, z_{1}\right) D_{\nu} W\left(z_{1}, z\right)\right] \psi(z n)
\end{aligned}
$$

where one has $\Gamma^{\mu}=\gamma^{\mu}, \gamma^{\mu} \gamma^{5}, \gamma^{\perp} \gamma^{\mu} \gamma^{5}$ for the unpolarized, helicity and transversity PDFs, respectively. $\Gamma_{0}$ is the incomplete Gamma function

$$
\Gamma_{0}(-i x)=\int_{0}^{1} \frac{d t}{t} e^{i x / t}
$$

The above twist-four contribution needs to be removed from the quasi-PDF to recover the leading-twist PDF. It also provides a possibility for practical computations on the lattice. However, as a multi-parton correlation involving more gauge links and covariant derivatives, its lattice computation is rather challenging and has not been carried out in any existing work yet. 
Another approach that has been used to estimate power corrections related to quark quasi-PDFs is the renormalon model (see (Beneke, 1999) for a comprehensive review). It is based on the observation that the perturbative expansion of the matching coefficient for the quasi-PDF diverges factorially with the loop order, implying that it is only well defined up to a power accuracy. This is known as the renormalon ambiguity, which must be cancelled by terms in the higher-twist contributions.

In (Braun et al., 2019), it was shown that the cancellation of renormalon ambiguity requires that the leading higher-twist or twist-four contribution takes the following form

$$
q_{4}\left(y, P^{z}, \mu\right)=\mu^{2} \int_{-1}^{1} \frac{d x}{|x|} D\left(\frac{y}{x}\right) q(x, \mu)+q_{4}^{\prime}\left(y, P^{z}, \mu\right),
$$

where the first term on the r.h.s. cancels the renormalon ambiguity from the leading-twist matching coefficient, and $q_{4}^{\prime}$ depends on $\mu$ at most logarithmically. Since the first term is to merely cancel similar contributions in the matching coefficient, it does not contribute to any physical observable. The renormalon model of power corrections (Beneke and Braun, 1995; Beneke et al., 1997; Braun et al., 2004; Dasgupta and Webber, 1996, 1997; Dokshitzer et al., 1996) is based on the assumption that, by replacing $\mu$ with a suitable nonperturbative scale, this contribution reflects the order and the functional form of actual power-suppressed contributions. This was known as "ultraviolet dominance" in Beneke, 1999; Beneke and Braun, 2000; Braun, 1995). Under this assumption, we obtain the following estimate,

$$
q_{4}\left(y, P^{z}, \mu\right)=\kappa \Lambda_{\mathrm{QCD}}^{2} \int_{-1}^{1} \frac{d x}{|x|} D\left(\frac{y}{x}\right) q(x, \mu),
$$

where $\kappa$ is a dimensionless coefficient of $\mathcal{O}(1)$ that cannot be fixed within theory and remains a free parameter.

A detailed analysis (Braun et al., 2019) showed that for the quasi-PDF we have

$$
\begin{aligned}
& q_{4}\left(y, P^{z}\right)=\frac{\kappa \Lambda_{\mathrm{QCD}}^{2}}{y^{2}(1-y)\left(P^{z}\right)^{2}} \\
& \times(1-y)\left[\int_{|y|}^{1} \frac{d x}{x}\left[\frac{x^{2}}{(1-x)_{+}}-2 x^{2}\right] q\left(\frac{y}{x}\right)+2 q(y)-|y| q^{\prime}(y)\right],
\end{aligned}
$$

where the first term in the integral was reproduced in a recent analysis of the renormalon effects in the quasiPDF (Liu and Chen, 2020). As one can see, the second row vanishes as $q(y)$ when $y \rightarrow 1$ if $\lim _{y \rightarrow 1} q(y) \sim(1-y)^{a}$ with $a>0$. This gives an estimate of the twist-four contribution on the r.h.s. of Eq. (33), which implies that the higher-twist contributions are enhanced as $1 / y^{2}$ and $1 /(1-y)$ for $y \sim 0$ and $y \sim 1$, respectively. Similar analysis can also be done for the pseudo-PDF. The above result can be used as a way to model the twist-four contribution with $\kappa$ as the only parameter.

\section{Orbital Angular Momemntum of Partons in the Proton}

Over the past three decades, much experimental and theoretical work has been done on the origin and structure of proton spin, which has been covered in depth in the review articles (Aidala et al., 2013; Bass, 2005; Deur et al., 2019; Filippone and Ji, 2001; Ji, 2017; Ji et al., 2020c; Leader and Lorcé, 2014).

In addition to the spin-dependent PDFs and TMDs, the GCPOs - in particular the GPDs - also play an important role in understanding the spin structure of the proton. Since GPDs describe the correlation between the transverse position and longitudinal momentum of quarks and gluons inside the proton, they offer a unique channel to study the orbital angular momentum (OAM) from experiments.

There are two widely known definitions of OAM in literature. One is the kinetic OAM in the gauge-invariant and frame-independent sum rule for the proton spin (Ji), $1997 \mathrm{a}, \mathrm{b})$, which is related to the first moment of twisttwo GPDs and can be calculated from the form factors of the symmetric QCD energy-momentum tensor. A review of the lattice calculations of kinetic OAM can be found in (Ji et al., 2020c). The other definition, which has a clear partonic interpretation in comparison to the kinetic OAM, is the canonical OAM in the naive partonic sum rule (Jaffe and Manohar, 1990) based on the free-field form of the QCD angular momentum,

$$
\begin{aligned}
\vec{J}= & \int d^{3} \xi \psi^{\dagger} \frac{\vec{\Sigma}}{2} \psi+\int d^{3} \xi \psi^{\dagger}[\vec{\xi} \times(-i \vec{\nabla})] \psi \\
& +\int d^{3} \xi \vec{E} \times \vec{A}+\int d^{3} \xi E^{i}(\vec{\xi} \times \vec{\nabla}) A^{i},
\end{aligned}
$$

where $i$ is the spatial Lorentz index. Except for the first one, the other three operators are gauge dependent, and their matrix elements are generally frame dependent. In high-energy scattering, there is one frame and gauge that are special: the IMF and light-front gauge, $A^{+}=0$. Therefore, the naive partonic sum rule for proton spin can be expressed as (Jaffe and Manohar, 1990)

$$
\frac{1}{2}=\frac{1}{2} \Delta \Sigma(\mu)+l_{q}^{z}(\mu)+\Delta G(\mu)+l_{g}^{z}(\mu),
$$

where $l_{q}^{z}(\mu)$ and $l_{g}^{z}(\mu)$ are the canonical OAM of the quark and gluon partons, respectively. Both $l_{q}^{z}$ and $l_{a}^{z}$ can be related to twist-three GPDs (Hatta, 2012; Hatta and Yoshida, 2012; Ji et al., 2013a), which can be accessed through spin-asymmetries in hard exclusive processes (Bhattacharya et al., 2018, 2017; Hatta et al., 2017; Ji et al., 2017a) (see the recent review (Ji et al., 2020c)).

To fully understand the partonic spin structure of the proton, one also needs to determine the quark and gluon canonical OAM, $l_{q}^{z}$ and $l_{g}^{z}$. LaMET allows extraction of $l_{q}^{z}$ and $l_{g}^{z}$ from lattice calculation in the same way as the gluon helicity that was reviewed in Sec. III.E. 
The quasi-partonic OAM operators can be chosen as the free-field operators fixed in gauges that belong to the universality class (Hatta et al., 2014). Their matrix elements $\tilde{l}_{q}^{z}$ and $\tilde{l}_{g}^{z}$ can be calculated from the off-forward matrix elements of the relevant energy-momentum tensors (Zhao et al., 2016), for example,

$$
\tilde{l}_{q}^{z}\left(2 S^{z}\right)=\left.\lim _{\Delta \rightarrow 0} \epsilon^{i j} \frac{\partial}{\partial i \Delta^{i}}\left\langle P^{\prime} S\left|\psi^{\dagger}(0) i \partial^{j} \psi(0)\right| P S\right\rangle\right|_{\vec{\nabla} \cdot \vec{A}=0} .
$$

where the kinematics is the same as Eq. (130).

Along with $\Delta G, \tilde{l}_{q}^{z}$ and $\tilde{l}_{g}^{z}$ can be matched to the partonic quantities defined in the Jaffe-Manohar sum rule through the factorization formulas,

$$
\begin{gathered}
\tilde{l}_{q}^{z}\left(P^{z}, \mu\right)=P_{q q} l_{q}^{z}(\mu)+P_{g q} l_{g}^{z}(\mu) \\
\quad+p_{q q} \Delta \Sigma(\mu)+p_{g q} \Delta G(\mu)+\ldots, \\
\tilde{l}_{g}^{z}\left(P^{z}, \mu\right)=P_{q g} l_{q}^{z}(\mu)+P_{g g} l_{g}^{z}(\mu) \\
+p_{q g} \Delta \Sigma(\mu)+p_{g g} \Delta G(\mu)+\ldots,
\end{gathered}
$$

where $\cdots$ are power corrections suppressed by the momentum $P^{z}$, and the one-loop matching coefficients in front of each term on the r.h.s. have been calculated in the Coulomb gauge (Ji et al., 2015c). Since the quasipartonic operators are gauge-variant and need to be fixed in a particular gauge, they can mix with new operators that are not allowed by Lorentz or gauge symmetries. For example, the gauge-dependent potential angular momentum $\psi^{\dagger}(\vec{r} \times \vec{A}) \psi$ comes into play Ji et al., 2016; Wakamatsu, 2014). Such mixings must be taken into account in lattice renormalization to have a controlled calculation of the canonical OAM.

Apart from the above approach, it has also been proposed to calculate the ratio of $l_{q}^{z}$ and the valence quark number from the derivatives of off-forward matrix elements of staple-shaped quark Wilson line operators (Engelhardt, 2017), whose definition can be found in Eq. (195) below. The first lattice calculations with this approach have been carried out in (Engelhardt, 2017; Engelhardt et al., 2018), which shows different size of effects between the kinetic and canonical OAM. For systematic improvement in this calculation, one should include the matching of such matrix elements to the physical $l_{q}^{z}$ in the limit when the transverse separation of the quark fields approaches zero.

For the transverse polarization, it is natural to define a twist-two partonic transverse angular momentum density of quarks (Hoodbhov et al., 1999b; Ji et al., 2012; Ji and Yuan, 2020; Ji et al., 2020c),

$$
J_{\perp}^{q}(x)=x\left[q(x)+E_{q}(x)\right] / 2,
$$

and similarly for the gluons, where $q(x)$ is the unpolarized quark/antiquark distributions, and $E_{q, g}(x)$ is the GPDs defined earlier in this section. Thus to get a simple partonic picture of the proton transverse spin from the first principles, it is important to calculate the GPD $E(x)$ using LaMET.

\section{TRANSVERSE-MOMENTUM DEPENDENT PDFS}

The transverse-momentum-dependent (TMD) parton distribution functions (TMDPDFs) are a natural generalization of the collinear PDFs to include both longitudinal and transverse momentum of partons. They are in principle probability distributions $f_{i}\left(x, \vec{k}_{\perp}, \sigma\right)$ of finding a parton of given species $i$, longitudinal and transverse momentum $\left(x P^{+}, \vec{k}_{\perp}\right)$, and polarization $\sigma$ inside the hadron state. TMDPDFs are playing an increasingly important role in understanding the partonic structure of hadrons and high-energy scattering.

The TMD parton densities were firstly introduced by Collins and Soper in 1980s (Bodwin, 1985; Collins and Soper, 1981, 1982a; Collins et al., 1983, $1985 \mathrm{a}, \mathrm{b})$ to understand the Drell-Yan (DY) and $e^{+} e^{-}$ annihilation process, and generalized in (Ji et al., 2004, 2005) to semi-inclusive deep-inelastic scattering(SIDIS) process. The TMD factorization has been reanalyzed in the framework of SCET in which modes are made manifest by effective fields (Bauer et al., 2001, 2002; Becher and Neubert, 2011; Chiu et al., 2012; Echevarría et al., 2013; Echevarria et al., 2012; Manohar and Stewart, 2007). Various TMD factorization formalisms finally converged to the standard one where a scheme-independent TMDPDF can be defined (Collins and Rogers, 2017, 2013; Echevarría et al., 2013).

The TMD parton densities are important in understanding the experimental processes where the transverse momenta of final state particles are measured. For example, in DY pair and $W, Z$ production it is known that the differential cross section $d \sigma / d Q_{T}^{2}$ normally peaks at relatively small transverse momentum. For $Q \sim 10 \mathrm{GeV}$, the peak is typically located at $Q_{\perp} \sim 1 \mathrm{GeV}$ where nonperturbative effects are important (Collins et al., 1985b). A good knowledge of TMD parton densities is therefore crucial for the determination of the cross sections and precision test of perturbative QCD predictions.

Besides their importance in understanding the highenergy experimental data, the TMD parton densities are also important by themselves for their crucial role in describing hadron structures. With them, one can simultaneously study the fast-moving collinear physics through the longitudinal $x$-dependencies, and the nonperturbative effect from the transverse $\vec{k}_{\perp}$-dependencies. Moreover, the TMDPDFs are sensitive to effects such as soft radiations. Therefore, the physics in the presence of transverse degrees of freedom is rather rich. This is particularly true in studies of spin-dependent phenomena where one can define various TMDPDFs through Lorentz decompositions (see Sec. V.B). One 
example is the Sivers function for a transversely polarized proton, $\epsilon_{i j} k_{\perp}^{i} S_{\perp}^{i} f_{1 T}^{\perp}\left(x, k_{\perp}\right)$, which is naive-timereversal odd and is predicted to change sign between the DY and SIDIS processes (Collins, 2011a). Similar properties also exist in the Boer-Mulders function (Boer and Mulders, 1998) concerning a transverselypolarized parton distribution in an unpolarized hadron. These two functions are related to the single transverse spin asymmetry. If we generalize the TMDPDFs to include the impact parameter dependence, we can further define the Wigner function, the parton orbital angular momentum distributions, etc (Belitsky et al., 2004; Lorce et al., 2012). Therefore, the TMDPDFs allow for a more complete and refined 3D description (or tomography) of the hadron structure (Boer et al., 2011; Burkardt, 2000). The 3D tomography of the proton is a major physical goal of the EIC program. The TMDPDFs are also important in understanding small- $x$ physics (Balitsky, 1996; Balitskv and Lipatov, 1978; Kovchegov, 1999; Kovchegov and Levin, 2012; Kuraev et al., 1977).

Our current knowledge on TMDPDFs mainly comes from fitting to the experimental data (Bacchetta et al., 2019, 2017; Bertone et al., 2019; Echevarria et al., 2014; Kang et al., 2016; Konvchev and Nadolsky, 2006; Landrv et al., 2001; Scimemi and Vladimirov, 2018a, 2019; (Sun et al., 2018). This is, however, rather primitive due to the paucity of data. Although the future EIC will make up the gap and produce more data for TMD measurements, it is still important to develop firstprinciple methods for the determination of nonperturbative TMDPDFs, which can serve as a test or provide useful inputs to constrain the global fits. LaMET provides a systematic way to extract TMDPDFs from the lattice calculations. Early studies (Ebert et al., 2019a, b; Ji et al., 2019a, 2015b) have tried to construct a quasiTMDPDF on the lattice, but its relation to the physical TMDPDF is expected to be nonperturbative due to complications in the soft function (Ebert et al., 2019b). The recent works in (Ji et al., 2019b, 2020a) provide a formulation to calculate the soft function so that a perturbative matching formula can be established between the quasiand physical TMDPDFs, allowing for a complete determination of the latter from lattice QCD. In this section we review the application of LaMET to the nonperturbative TMDPDFs. The investigation is still in its early stage and a lot remains to be explored, particularly in lattice calculations and matching.

In the first subsection we introduce the TMDPDFs and discuss the associated rapidity divergences. In the following subsections, we define the quasi-TMDPDFs or TMD momentum distributions in a proton of finite momentum, and study their momentum RGEs and UV renormalization properties. In the process, we introduce the off-lightcone soft functions. We then present the factorization of the quasi-TMDPDFs into the light-cone TMDPDFs and the off-light-cone soft function, where various one-loop results and the relevant RGEs are also given. The properties of the off-light-cone soft function are discussed in the last subsection, where it is shown to be related to the form factor of a pair of charged color sources, which paves the way for its calculation on a Euclidean lattice.

\section{A. Introduction to TMDPDFs and Rapidity Divergence}

As explained in Sec. [I] we can define various TMDPDFs by choosing different gauge-links between the quark or gluon bilinears. The one relevant to highenergy phenomena is defined with light-like Wilson lines. The links represent the propagation of high-energy colorcharged particles, and are crucial in forming gaugeinvariant nonlocal operators (Belitsky et al., 2003). As argued in previous sections, such operators are the result of an EFT description (more explicitly so in SCET) arising from taking the infinite-momentum limit of the proton. Thus, it is natural to expect that they require additional regularization and renormalization.

Let us take the non-singlet quark unpolarized TMD$\mathrm{PDF}$ as an example. Without the field theoretic subtleties, the distribution is

$$
\begin{aligned}
& f\left(x, \vec{k}_{\perp}\right)=\frac{1}{2 P^{+}} \int \frac{d \lambda}{2 \pi} \frac{d^{2} \vec{b}_{\perp}}{(2 \pi)^{2}} e^{-i \lambda x+i \vec{k}_{\perp} \cdot \vec{b}_{\perp}} \\
& \times\left\langle P\left|\bar{\psi}\left(\lambda n / 2+\vec{b}_{\perp}\right) \gamma^{+} \mathcal{W}_{n}\left(\lambda n / 2+\vec{b}_{\perp}\right) \psi(-\lambda n / 2)\right| P\right\rangle
\end{aligned}
$$

where $\mathcal{W}_{n}\left(\lambda n+\vec{b}_{\perp}\right)$ is the staple-shaped gauge-link of the form

$$
\begin{aligned}
\mathcal{W}_{n}(\xi) & =W_{n}^{\dagger}(\xi) W_{\perp} W_{n}(-\xi \cdot p n) \\
W_{n}(\xi) & =\mathcal{P} \exp \left[-i g \int_{0}^{-\infty} d \lambda n \cdot A(\xi+\lambda n)\right]
\end{aligned}
$$

along the light-cone direction $n^{\mu}$, as shown in Fig. 10, The $W_{\perp}$ is a transverse gauge-link at light-cone infinity to maintain gauge-invariance.. If one uses LFQ and ignores the transverse gauge-link, the above distribution is just $\left\langle P\left|b^{\dagger}\left(x, \vec{k}_{\perp}\right) b\left(x, \vec{k}_{\perp}\right)\right| P\right\rangle$ for $x>0$, as expected.

However, there are a number of qualifications in the above definition. First, the light-like gauge-links $\mathcal{W}_{n}$ are chosen to be past-pointing in accordance with the DY kinematics, but for SIDIS they should be chosen as future-pointing, as shown in Fig. 10, For unpolarized TMDPDFs there is no distinction between the two choices, but for spin-dependent TMDPDFs there are physical consequences associated with the direction of gauge-links.

Second, there exists a new type of divergence associated with the infinitely-long light-like gauge-links. These divergences are due to radiation of gluons collinear to the light-like gauge-link and cannot be regularized by the 


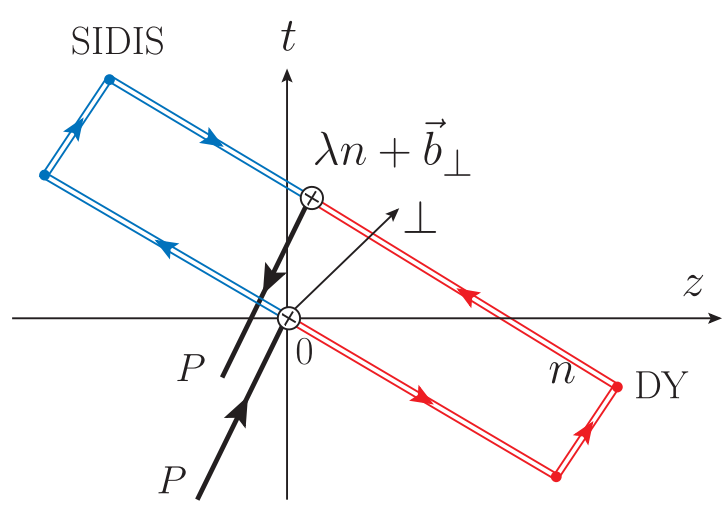

FIG. 10: The space-time picture of TMDPDF for DY and SIDIS process. The circled crosses denote the quark-link vertices. Notice that the vertices are placed at $\lambda n+\vec{b}_{\perp}$ and 0 which gives the same result as the symmetric choice in Eq. (165).

standard UV regulators. An example is the following integral in dimensional regularization (DR) (Ebert et al., 2019b),

$$
I=\int d k^{+} d k^{-} \frac{f\left(k^{+} k^{-}\right)}{\left(k^{+} k^{-}\right)^{1+\epsilon}}=\frac{1}{2} \int \frac{d y}{y} \int d m^{2} \frac{f\left(m^{2}\right)}{m^{2+2 \epsilon}},
$$

where $m^{2}=k^{+} k^{-}$and $y=k^{+} / k^{-}$is the rapidity-related variable. The divergences in $y$ arise from large and small $y$ where the integral is unregulated. The contribution from $k^{+}=0$ is called the light-zero mode in LFQ, where it is also called light-cone divergence which causes considerable problems.

To regulate the light-cone or rapidity divergences, a number of methods have been introduced in the literature (for a review see (Ebert et al. 2019b)). They can be put into two classes: on-light-cone regulators and off-light-cone regulators. In the former case, the gauge-links are kept along the light-cone direction $n^{\mu}$ after regularization. For example, the so-called $\delta$ regulator (Echevarria et al., 2016a b) regularizes the gauge-link as:

$$
\begin{aligned}
& \left.W_{n}(\xi) \rightarrow W_{n}(\xi)\right|_{\delta^{-}} \\
& =\mathcal{P} \exp \left[-i g \int_{0}^{-\infty} d \lambda A^{+}(\xi+\lambda n) e^{-\frac{\delta^{-}}{2 p^{+}}|\lambda|}\right],
\end{aligned}
$$

and similarly for the conjugate direction. The $\delta$ regulator breaks gauge-invariance, but preserves the boost invariance $\delta^{ \pm} \rightarrow e^{ \pm Y} \delta^{ \pm}$where $Y$ is the rapidity of the Lorentz boost. Other on-light-cone regulators include the exponential regulator ( $\mathrm{Li}$ et al. 2016), $\eta$ regulator (Chiu et al., 2012), analytical regulator (Becher and Neubert, 2011), etc. In the remainder of this section, we will use the $\delta$ regulator as a representative whenever we need an on-light-cone regulator.

The off-light-cone regulator was introduced in (Collins, 2011a; Collins and Soper, 1981; Ji et al., 2004,
2005), and also used in (Ji et al., 2005). This type of regulator chooses off-light-cone directions to avoid the rapidity divergence. One can choose, for instance, to deform the gauge-links into the space-like region:

$$
n \rightarrow n_{Y}=n-e^{-2 Y} \frac{p}{\left(p^{+}\right)^{2}} .
$$

Here $Y$ plays the role of a rapidity regulator, as when $Y \rightarrow \infty, n_{Y} \rightarrow n$. In certain cases one can also deform $n_{Y}$ into time-like region (Collins and Metz, 2004).

The on-light-cone regulators are consistent with the spirit of parton physics, and therefore are useful to define COM-momentum-independent parton densities. The off-light-cone regulators, on the other hand, follow a similar spirit as LaMET, and therefore can be exploited for practical lattice QCD calculations, as we shall see in the next subsection.

To avoid light-cone divergences, from now on we include the rapidity regulator in the definition of the lightcone TMDPDFs. Using the same label $f$ for the TMDPDFs in both momentum and coordinate spaces, we have

$$
\begin{aligned}
& f\left(\lambda, b_{\perp}, \mu, \delta^{-} / P^{+}\right) \\
& =\left\langle P\left|\bar{\psi}\left(\lambda n / 2+\vec{b}_{\perp}\right) \hbar \mathcal{W}_{n}\left(\lambda n / 2+\vec{b}_{\perp}\right)\right|_{\delta^{-}} \psi(-\lambda n / 2) \mid P\right\rangle,
\end{aligned}
$$

where $\mu$ is the $\overline{\mathrm{MS}}$ scale for UV renormalization. Due to rotational invariance, the bare TMDPDF defined above is a function of $b_{\perp}=\left|\vec{b}_{\perp}\right|$, so we have omitted the vector arrow for $\vec{b}_{\perp}$ in $f$ and will do so throughout the discussion for the soft functions, quasi-TMDPDFs, etc. The subscript $\delta^{-}$denotes that the staple-shaped gauge-link $\mathcal{W}$ is regulated by the $\delta$ regulator in the light-cone minus direction. $f$ diverges logarithmically as $\delta^{-} \rightarrow 0$, and the finite part also depends on the rapidity regulator. To define the physical TMDPDF, we need to remove all divergences and rapidity regularization scheme dependencies in $f$, in a way similar to removing UV divergences in physical quantities.

The rapidity divergence for TMDPDFs can be removed by the soft function, which also plays an important role in TMD factorization. Intuitively, the soft function represents a cross section for fast-moving charged particles emitting soft gluons into final states. It has rapidity divergence associated with the light-cone direction, which is ultimately related to the mass singularity. The TMD soft function corresponding to Drell-Yan process is defined (Collins, 2011b; Echevarria et al., 2016b) as

$$
\begin{aligned}
& S\left(b_{\perp}, \mu, \delta^{+}, \delta^{-}\right) \\
& =\frac{\operatorname{Tr}\left\langle\left.\left.\left. 0\left|\overline{\mathcal{T}} W_{p}\left(\vec{b}_{\perp}\right)\right|_{\delta^{+}} W_{n}^{\dagger}\left(\vec{b}_{\perp}\right)\right|_{\delta^{-}} \mathcal{T} W_{n}(0)\right|_{\delta^{-}} W_{p}^{\dagger}(0)\right|_{\delta^{+}} \mid 0\right\rangle}{N_{c}} \\
& =\frac{\operatorname{tr}\left\langle\left. 0\left|\mathcal{W}_{n}\left(\vec{b}_{\perp}\right)\right|_{\delta^{+}} \mathcal{W}_{p}^{\dagger}\left(\vec{b}_{\perp}\right)\right|_{\delta^{-}} \mid 0\right\rangle}{N_{c}},
\end{aligned}
$$

where $\mathcal{T} / \overline{\mathcal{T}}$ stands for time/anti-time ordering. The first equality defines the soft function in terms of cut-diagrams 


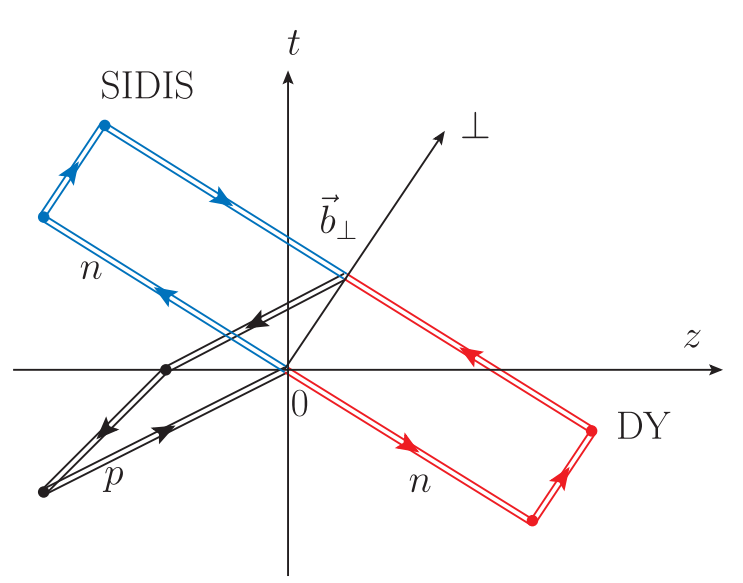

FIG. 11: The soft function $S\left(b_{\perp}, \mu, \delta^{+}, \delta^{-}\right)$as space-time Wilson-loop arising in the factorization of DY and SIDIS process.

as an amplitude square. Since the soft function for DY process is independent of time ordering, one can also define it with a single time ordering or no time ordering, leading to the second equality. The staple-shaped gaugelink $\mathcal{W}_{n}$ is defined in Eq. (166), while the staple-shaped gauge-link $\mathcal{W}_{p}$ is defined similarly as:

$$
\begin{aligned}
& \mathcal{W}_{p}(\xi)=W_{p}^{\dagger}(\xi) W_{\perp} W_{p}(0) \\
& W_{p}(\xi)=\mathcal{P} \exp \left[-i g \int_{0}^{-\infty} d \lambda p \cdot A(\xi+p \lambda)\right] .
\end{aligned}
$$

The soft function is shown in Fig. 11] as a Wilson loop in Minkowski space.

If the rapidity divergences are multiplicative, one can use $S$ as the rapidity renormalization factor for the TMDPDF defined in Eq. (165). In on-light-cone schemes such as the $\delta$ regularization, it has been argued in (Vladimirov, 2018) based on conformal transformation that the rapidity divergences are indeed multiplicative in position space. For each of the staple-shaped light-like gauge-links, the rapidity divergence is proportional to $\exp \left[-(1 / 2) K\left(b_{\perp}, \mu\right) \ln \left(\mu^{2} / 2\left(\delta^{ \pm}\right)^{2}\right)\right]$ where $K\left(b_{\perp}, \mu\right)$ is the nonperturbative Collins-Soper evolution kernel (Collins and Soper, 1981). Thus at small $\delta^{ \pm}$, we can write

$$
S\left(b_{\perp}, \mu, \delta^{+}, \delta^{-}\right)=e^{\ln \frac{\mu^{2}}{2 \delta^{+} \delta^{-}} K\left(b_{\perp}, \mu\right)+\mathcal{D}_{2}\left(b_{\perp}, \mu\right)},
$$

where $\mathcal{D}_{2}\left(b_{\perp}, \mu\right)$ is a $b_{\perp}$-dependent but rapidityindependent function. Notice that our definitions of $\delta^{ \pm}$ differ from those in Ref. (Echevarria et al., 2016b) by a factor of $\sqrt{2}$ due to our normalization of light-cone vectors.

The soft-function in $\delta$ regularization satisfies the renormalization group equation

$$
\begin{aligned}
& \mu^{2} \frac{d}{d \mu^{2}} \ln S\left(b_{\perp}, \mu, \delta^{+}, \delta^{-}\right) \\
& =-\Gamma_{\text {cusp }}\left(\alpha_{s}\right) \ln \frac{\mu^{2}}{2 \delta^{+} \delta^{-}}+\gamma_{s}\left(\alpha_{s}\right),
\end{aligned}
$$

where $\Gamma_{\text {cusp }}\left(\alpha_{s}\right)$ is the light-like cusp anomalous dimension (Korchemsky and Radyushkin, 1987; Polyakov, 1980) and the $\gamma_{s}\left(\alpha_{s}\right)$ is the soft anomalous dimension (Korchemskaya and Korchemsky, 1992). The Collins-Soper kernel and the rapidity-independent part $\mathcal{D}_{2}$ satisfy the RGEs:

$$
\begin{array}{r}
\mu^{2} \frac{d}{d \mu^{2}} K\left(b_{\perp}, \mu\right)=-\Gamma_{\text {cusp }}\left(\alpha_{s}\right), \\
\mu^{2} \frac{d}{d \mu^{2}} \mathcal{D}_{2}\left(b_{\perp}, \mu\right)=\gamma_{s}\left(\alpha_{s}\right)-K\left(b_{\perp}, \mu\right) .
\end{array}
$$

At one-loop, the soft function $S\left(b_{\perp}, \mu, \delta^{+}, \delta^{-}\right)$is given by (Echevarría et al., 2013) :

$$
\begin{aligned}
& S\left(b_{\perp}, \mu, \delta^{+}, \delta^{-}\right) \\
& =1+\frac{\alpha_{s} C_{F}}{2 \pi}\left(L_{b}^{2}-2 L_{b} \ln \frac{\mu^{2}}{2 \delta^{+} \delta^{-}}+\frac{\pi^{2}}{6}\right),
\end{aligned}
$$

where $L_{b}=\ln \left(\mu^{2} b_{\perp}^{2} e^{2 \gamma_{E}} / 4\right)$. Therefore, we have at the leading order,

$$
\begin{aligned}
K\left(b_{\perp}, \mu\right) & =-\frac{\alpha_{s} C_{F}}{\pi} L_{b}, \\
\mathcal{D}_{2}\left(b_{\perp}, \mu\right) & =\frac{\alpha_{s} C_{F}}{2 \pi}\left(L_{b}^{2}+\frac{\pi^{2}}{6}\right),
\end{aligned}
$$

and $\Gamma_{\text {cusp }}=\alpha_{s} C_{F} / \pi+\mathcal{O}\left(\alpha_{s}^{2}\right), \gamma_{s}=\mathcal{O}\left(\alpha_{s}^{2}\right)$. It is worth pointing out that $K$ (Li and Zhu, 2017; Vladimirov, 2017) and $\mathcal{D}_{2}$ (Li and Zhu, 2017) are known to 3-loop order in the exponential regularization scheme.

With the above soft function, we can take its square root to perform rapidity renormalization for the bare TMD correlator. The square root can be explained as follows: $S$ contains two staples, while $f$ contains one, thus the rapidity divergences as well as scheme dependencies in $S$ are twice as those in $f$. This leads to the following definition of the renormalized physical TMDPDF (Collins and Rogers, 2013; Echevarría et al., 2013):

$$
f^{\mathrm{TMD}}\left(x, b_{\perp}, \mu, \zeta\right)=\lim _{\delta^{-} \rightarrow 0} \frac{f\left(x, b_{\perp}, \mu, \delta^{-} / P^{+}\right)}{\sqrt{S\left(b_{\perp}, \mu, \delta^{-} e^{2 y_{n}}, \delta^{-}\right)}},
$$

where the rapidity scale reads

$$
\zeta=2\left(x P^{+}\right)^{2} e^{2 y_{n}} .
$$

The rapidity dependence in the numerator of the right-hand side of Eq. (182) has the form $\exp \left[-\frac{1}{2} K\left(b_{\perp}, \mu\right) \ln \frac{\left(\delta^{-}\right)^{2}}{\left(x P^{+}\right)^{2}}\right]$, while in the denominator it behaves as $\exp \left[\frac{1}{2} K\left(b_{\perp}, \mu\right) \ln \frac{\mu^{2}}{2\left(\delta^{-}\right)^{2} e^{2 y_{n}}}\right]$. The $\delta^{-}$dependence thus cancels out in the ratio, leaving a dependence on the rapidity scale as $\exp \left[-\frac{1}{2} K\left(b_{\perp}, \mu\right) \ln \frac{\mu^{2}}{2\left(x P^{+}\right)^{2} e^{2 y_{n}}}\right]$, which is controlled by the so-called Collins-Soper evolution equation:

$$
2 \zeta \frac{d}{d \zeta} \ln f^{\mathrm{TMD}}\left(x, b_{\perp}, \mu, \zeta\right)=K\left(b_{\perp}, \mu\right) .
$$


The $\zeta$-dependence comes from the initial-state quark radiation and is intrinsically nonperturbative for large $b_{\perp}$. $f^{\mathrm{TMD}}\left(x, b_{\perp}, \mu, \zeta\right)$ is the standard object to be matched to in LaMET.

We should emphasize that although $f^{\text {TMD }}$ is free from rapidity divergences, it does contain soft radiation from the charged particles in the initial state. This can be seen clearly by considering Feynman diagrams for the unsubtracted $f$ and applying soft approximation to gluons. "One-half" of the soft contribution in $f$ is subtracted to define the physical $f^{\text {TMD }}$ due to the requirement of factorization of physical processes. The remaining soft radiation also has a natural rapidity cut-off associated with $\ln \left(x P^{+}\right)$, reflected in the $\zeta$-dependence. What is remarkable, however, is that $f^{\mathrm{TMD}}$ is rapidity-regulator independent. Although a general proof to all orders in perturbation theory is beyond the scope of this review, it is due to factorization and exponentiation of the soft physics in $f$ and thus the scheme cancellation can be done systematically in the exponent. It worth mentioning that in old-fashioned or SCET-like approaches, one can define the "subtracted" TMDPDF or "beams functions" that contains only collinear physics. However, they are generically scheme dependent and must be combined with an extra soft functions in factorization theorems. At oneloop level, the scheme-independent one-loop TMDPDF for an external quark state reads,

$$
\begin{aligned}
& f^{\mathrm{TMD}}\left(x, b_{\perp}, \mu, \zeta\right)=\delta(1-x) \\
& +\frac{\alpha_{s} C_{F}}{2 \pi} F\left(x, \epsilon_{\mathrm{IR}}, b_{\perp}, \mu\right) \theta(x) \theta(1-x)+\frac{\alpha_{s} C_{F}}{2 \pi} \delta(1-x) \\
& \times\left[-\frac{1}{2} L_{b}^{2}+\left(\frac{3}{2}-\ln \frac{\zeta}{\mu^{2}}\right) L_{b}+\frac{1}{2}-\frac{\pi^{2}}{12}\right],
\end{aligned}
$$

where

$$
F\left(x, \epsilon_{\mathrm{IR}}, b_{\perp}, \mu\right)=\left[-\left(\frac{1}{\epsilon_{\mathrm{IR}}}+L_{b}\right) \frac{1+x^{2}}{1-x}+1-x\right]_{+} .
$$

Two-loop order results for the TMDPDFs can be found in (Catani and Grazzini, 2012; Catani et al., 2012; Echevarria et al., 2016c; Gehrmann et al., 2014; Lübbert et al., 2016; Luo et al., 2019) and three-loop order results can be found in (Luo et al., 2020).

The physical TMDPDF also satisfies the RG equation,

$$
\begin{aligned}
\gamma_{\mu}(\mu, \zeta) & =\mu^{2} \frac{d}{d \mu^{2}} \ln f^{\mathrm{TMD}}\left(x, b_{\perp}, \mu, \zeta\right) \\
& \equiv \frac{1}{2} \Gamma_{\text {cusp }}\left(\alpha_{s}\right) \ln \frac{\mu^{2}}{\zeta}-\gamma_{H}\left(\alpha_{s}\right),
\end{aligned}
$$

where $\gamma_{H}$ is called the hard anomalous dimension. At one-loop, the cusp and hard anomalous dimensions read

$$
\Gamma_{\text {cusp }}\left(\alpha_{s}\right)=\frac{\alpha_{s} C_{F}}{\pi} ; \quad \gamma_{H}\left(\alpha_{s}\right)=-\frac{3 \alpha_{s} C_{F}}{4 \pi} .
$$

Recently the cusp anomalous dimension have been calculated to 4-loops (Henn et al., 2019; von Manteuffel et al., 2020).

Combining the RGE and the rapidity evolution equation for the TMDPDF, one obtains the consistency condition :

$$
\mu^{2} \frac{d}{d \mu^{2}} K\left(b_{\perp}, \mu\right)=-2 \zeta \frac{d}{d \zeta} \gamma_{\mu}(\mu, \zeta)=-\Gamma_{\text {cusp }}\left(\alpha_{s}(\mu)\right),
$$

from which one finds a resummed form for the CollinsSoper kernel:

$$
K\left(b_{\perp}, \mu\right)=-2 \int_{1 / b_{\perp}}^{\mu} \frac{d \mu^{\prime}}{\mu^{\prime}} \Gamma_{\text {cusp }}\left(\alpha_{s}\left(\mu^{\prime}\right)\right)+K\left(\alpha_{s}\left(1 / b_{\perp}\right)\right) .
$$

Here $K\left(\alpha_{s}\left(1 / b_{\perp}\right)\right)$ contains both perturbative and nonperturbative contributions. The TMDPDFs at different scales are then related by

$$
\begin{aligned}
& f^{\mathrm{TMD}}\left(x, b_{\perp}, \mu, \zeta\right)=f^{\mathrm{TMD}}\left(x, b_{\perp}, \mu_{0}, \zeta_{0}\right) \\
& \times \exp \left[\int_{\mu_{0}}^{\mu} \frac{d \mu^{\prime}}{\mu^{\prime}} \gamma_{\mu}\left(\mu^{\prime}, \zeta_{0}\right)\right] \exp \left[\frac{1}{2} K\left(b_{\perp}, \mu\right) \ln \frac{\zeta}{\zeta_{0}}\right] .
\end{aligned}
$$

The double-scale evolution in the $\mu-\zeta$ plane for phenomenology has been recently studied in (Scimemi and Vladimirov, 2018b). With the scheme-independent physical TMDPDF defined above, the DY cross section at $s=\left(P_{A}+P_{B}\right)^{2}$ and small $Q_{\perp}$ can be factorized as

$$
\begin{aligned}
& \frac{d \sigma}{d Q_{\perp}^{2}}=\int d x_{A} d x_{B} d^{2} b_{\perp} e^{i \vec{b}_{\perp} \cdot \vec{Q}_{\perp}} \hat{\sigma}\left(x_{A} x_{B} s, \mu\right) \\
& \times f_{A}^{\mathrm{TMD}}\left(x_{A}, b_{\perp}, \mu, \zeta_{A}\right) f_{B}^{\mathrm{TMD}}\left(x_{B}, b_{\perp}, \mu, \zeta_{B}\right)+\ldots
\end{aligned}
$$

The rapidity scales satisfy $\zeta_{A} \zeta_{B}=Q^{4} \equiv\left(x_{A} x_{B} s\right)^{2}$. The remaining term at large but finite $Q^{2}$ are called power corrections or "higher-twist" contributions. A detailed study of the power corrections to TMD factorization is beyond the scope of this review. Without mention we will omit all the power-corrections in equations. The QCD part of the hard cross section $\hat{\sigma}$ at one-loop level reads

$$
\hat{\sigma}\left(x_{A}, x_{B}\right)=\left|1+\frac{\alpha_{s} C_{F}}{4 \pi}\left(-L_{Q}^{2}+3 L_{Q}-8+\frac{\pi^{2}}{6}\right)\right|^{2},
$$

where $L_{Q}=\ln \frac{-Q^{2}-i 0}{\mu^{2}}$, and the result is now known up to three loops (see (Baikov et al., 2009; Gehrmann et al., 2010; Lee et al., 2010; Moch et al., 2005) and the references therein). Similarly for the SIDIS process we have

$$
\begin{aligned}
\frac{d \sigma}{d Q_{\perp}^{2}}= & \int d x d z d^{2} b_{\perp} e^{i \vec{b}_{\perp} \cdot \vec{Q}_{\perp}} H(x, z, \mu, Q) \\
& \times f^{\mathrm{TMD}}\left(x, b_{\perp}, \mu, \zeta_{A}\right) d^{\mathrm{TMD}}\left(z, b_{\perp}, \mu, \zeta_{B}\right)
\end{aligned}
$$

where $d^{\mathrm{TMD}}\left(z, b_{\perp}, \mu, \zeta_{B}\right)$ is the TMD fragmentation function and $H$ is the hard kernel. 


\section{B. Lattice Quasi-TMDPDFs and Matching}

Before LaMET, there had been efforts to access TMD physics from lattice QCD by calculating the ratios of the $x$-moments of TMDPDFs (Engelhardt et al., 2016; Hagler et al., 2009; Musch et al., 2012, 2011; Yoon et al., 2017), which are free from complications associated with the soft function and can be compared to certain experimental observables. In LaMET, we are more interested in obtaining the full $x$ and $\vec{k}_{\perp}$ dependence of the TMDPDFs (Ebert et al., 2019a, b; Ji et al., 2019a, b, 2020a, 2015b). Therefore, a proper treatment of the soft function subtraction and matching is essential. The earliest suggestion of a bent soft function in (Ji et al., 2015b) and the follow-up work (Ebert et al., 2019b) has the correct IR logarithms at one-loop order, but this is expected to break down at higher-loop orders (Ji et al., 2020a), thus not allowing for a perturbative matching. Another suggestion which uses a naive rectangle-shaped Wilson loop (Ebert et al., 2019b; Ji et al., 2019a) does not possess the correct IR physics, either. Nevertheless, in (Ebert et al., 2019a) important progress was made for calculating the nonperturbative Collins-Soper kernel $K\left(b_{\perp}, \mu\right)$ from the ratio of quasi-TMDPDFs at two different large momenta. Recently, some of the authors showed (Ji et al., 2019b, 2020a) that the quasi-TMDPDF combined with a reduced soft function capture the correct IR physics to all-orders and thus allow for a perturbative matching to the physical TMDPDF.

To construct such quasi-TMDPDFs, the collinear part can be treated in a way similar to the collinear PDFs, while the soft piece is more challenging. Our starting point is that the physical $f^{\mathrm{TMD}}$ is independent of the rapidity regulator, so one can use a scheme in which the gauge-links in both $f$ and $S$ are off the light-cone, such as that used in (Collins, 2011a). In this case, one can use Lorentz symmetry to boost the staple-shaped gauge-link $\mathcal{W}_{n}$ in $f$ to a purely space-like staple with no time dependence. However, one can only use this trick for one of the staples in $S$, say $\mathcal{W}_{n}$, whereas the other one $\mathcal{W}_{p}$ is still time-dependent. In other words, there is no way to get rid of the time dependence in $S$ entirely with Lorentz boost alone. This is natural because $S$ in fact represents the square of an $S$-matrix, which appears to be intrinsically Minkowskian. However, using the LaMET principle that time dependence of an operator can be simulated through external physical states at large momentum, we find that $S$ can indeed be calculated on the lattice in the off-light-cone scheme as a form factor. A detailed discussion will be given in the next subsection. Here we assume that this is true, and discuss the matching between quasiand physical TMDPDFs.

First, we define the quasi-TMDPDF with a staple-shaped gauge-link along the $z$ direction (Ebert et al. 2019b; Ji et al., 2019a, , 2015b) as

$$
\begin{aligned}
& \tilde{f}\left(\lambda, b_{\perp}, \mu, \zeta_{z}\right) \\
& =\lim _{L \rightarrow \infty} \frac{\left\langle P\left|\bar{\psi}\left(\frac{\lambda n_{z}}{2}+\vec{b}_{\perp}\right) \gamma^{z} \mathcal{W}_{z}\left(\frac{\lambda n_{z}}{2}+\vec{b}_{\perp} ; L\right) \psi\left(-\frac{\lambda n_{z}}{2}\right)\right| P\right\rangle}{\sqrt{Z_{E}\left(2 L, b_{\perp}, \mu\right)}},
\end{aligned}
$$

where the $\overline{\mathrm{MS}}$ renormalization is implied, and

$$
\begin{aligned}
& \mathcal{W}_{z}(\xi ; L)=W_{z}^{\dagger}(\xi ; L) W_{\perp} W_{z}\left(-\xi^{z} n_{z} ; L\right) \\
& W_{z}(\xi ; L)=\mathcal{P} \exp \left[-i g \int_{\xi^{z}}^{L} d \lambda n_{z} \cdot A\left(\vec{\xi}_{\perp}+n_{z} \lambda\right)\right]
\end{aligned}
$$

Here $\xi^{z}=-\xi \cdot n_{z}$ and $\zeta_{z}=\left(2 x P^{z}\right)^{2}$ is the Collins-Soper scale of the quasi-TMDPDF. $W_{\perp}$ is inserted at $z=L$ to maintain explicit gauge invariance. $\sqrt{Z_{E}\left(2 L, b_{\perp}, \mu, 0\right)}$ is the square root of the vacuum expectation value of a flat rectangular Euclidean Wilson-loop along the $n_{z}$ direction with length $2 L$ and width $b_{\perp}$ :

$$
Z_{E}\left(2 L, b_{\perp}, \mu\right)=\frac{1}{N_{c}} \operatorname{Tr}\left\langle 0\left|W_{\perp} \mathcal{W}_{z}\left(\vec{b}_{\perp} ; 2 L\right)\right| 0\right\rangle .
$$

Again, $\gamma^{z}$ can be replaced by $\gamma^{t}$ as in the collinear quasiPDF. For a depiction of $\tilde{f}$ and $Z_{E}$ see Fig. 12 ,

The purpose of the factor $Z_{E}$ is as follows. At large $L$, the naive quasi-TMD correlator in the numerator of Eq. 195) contains divergences that go as $e^{-L E\left(b_{\perp}, \mu\right)}$ where $E\left(b_{\perp}\right)$ is the ground state energy of a pair of static heavy-quarks. $E\left(b_{\perp}, \mu\right)=2 \delta m+V\left(b_{\perp}, \mu\right)$ contains both the linear divergent mass corrections $2 \delta m$ and the heavyquark potential $V\left(b_{\perp}, \mu\right)$ due to mutual interactions. In literature the $L V\left(b_{\perp}, \mu\right)$ part was sometimes called the "pinch pole singularity." Therefore, we introduce the square root of a rectangular Wilson-loop $Z_{E}\left(2 L, b_{\perp}, \mu\right)$ with twice the length to cancel all these divergences and guarantee the existence of the $L \rightarrow \infty$ limit after the subtraction. The introduction of $\sqrt{Z_{E}}$ also removes additional contributions from the transverse gauge link. An alternative approach to avoid the pinch-pole singularity was proposed in $(\mathrm{Li}, 2016)$. We should mention that although the $\sqrt{Z_{E}}$ subtraction removes all the linear divergences, the logarithmic UV divergences are still present. Therefore, a non-perturbative renormalization of $\tilde{f}$ on the lattice is still required, which has been studied in the RI/MOM scheme (Shanahan et al., 2019), and its matching to the $\overline{\mathrm{MS}}$ scheme has been calculated at one-loop order (Constantinou et al., 2019; Ebert et al., 2020b).

The quasi-TMDPDFs defined above satisfy the following RGE (Collins and Soper, 1981; Ji et al., 2019b, 2015b)

$$
\mu^{2} \frac{d}{d \mu^{2}} \ln \tilde{f}\left(x, b_{\perp}, \mu, \zeta_{z}\right)=\gamma_{F}\left(\alpha_{s}(\mu)\right),
$$

where $\gamma_{F}$ is the anomalous dimension for the heavy-tolight current in Sec. III.A This is due to the fact that the 

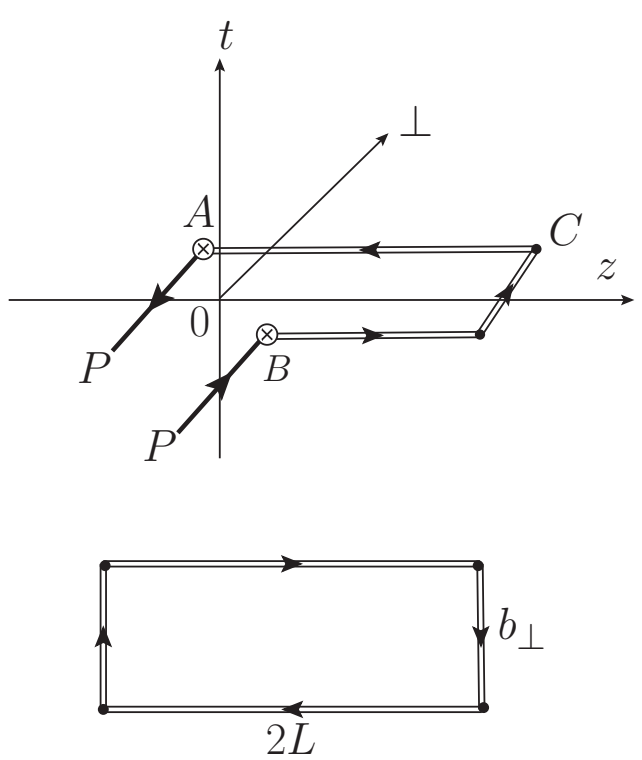

FIG. 12: The quasi-TMDPDF (upper) and the Euclidean Wilson-loop $Z_{E}\left(2 L, b_{\perp}, \mu, 0\right)$ (lower). In the figure,

$A=\lambda n_{z} / 2+\vec{b}_{\perp} / 2, B=-\lambda n_{z} / 2-\vec{b}_{\perp} / 2$ and $C=L n_{z}+\vec{b}_{\perp}$. The crosses denote the quark-link vertices.

quasi-TMDPDF, after the self-energy subtraction, contains only logarithmic UV divergences associated with quark-Wilson-line vertices. In the $\overline{\mathrm{MS}}$ scheme, the oneloop quasi-TMDPDF in an external quark state with momentum $\left(p^{z}, 0,0, p^{z}\right)$ reads (Ebert et al., 2019b; Ji et al., 2019a)

$$
\begin{aligned}
& \tilde{f}\left(x, b_{\perp}, \mu, \zeta_{z}\right)= \\
& 1+\frac{\alpha_{s} C_{F}}{2 \pi} F\left(x, \epsilon_{\mathrm{IR}}, b_{\perp}, \mu\right) \theta(x) \theta(1-x)+\frac{\alpha_{s} C_{F}}{2 \pi} \delta(1-x) \\
& \times\left[-\frac{1}{2} L_{b}^{2}+L_{b}\left(\frac{5}{2}-L_{z}\right)-\frac{3}{2}-\frac{1}{2} L_{z}^{2}+L_{z}\right],
\end{aligned}
$$

where $L_{z}=\ln \left(\zeta_{z} / \mu^{2}\right)$. As expected, the $L$ dependence has been cancelled in the large $L$ limit.

As there is no light-like gauge-link in $\tilde{f}$, no additional rapidity regulator is needed. Instead, there is an explicit dependence on the hadron momentum (or energy), which is similar to the momentum RGE for collinear quasiPDF. The momentum (rapidity) evolution equation for $\tilde{f}$ reads (Collins and Soper, 1981; Ji et al., 2019b, 2015b),

$$
P^{z} \frac{d}{d P^{z}} \ln \tilde{f}\left(x, b_{\perp}, \mu, \zeta_{z}\right)=K\left(b_{\perp}, \mu\right)+\mathcal{G}\left(\frac{\left(P^{z}\right)^{2}}{\mu^{2}}\right)
$$

where $\mathcal{G}\left(\zeta_{z} / \mu^{2}\right)$ is perturbative and $K\left(b_{\perp}, \mu\right)$ is the Collins-Soper kernel. A similar equation was proven for off-light-cone TMD-fragmentation functions in (Collins and Soper, 1981). From this equation, it is clear that a correct matching to $f^{\mathrm{TMD}}\left(x, b_{\perp}, \mu, \zeta\right)$ with arbitrary $\zeta$ must include $K\left(b_{\perp}, \mu\right)$ to compensate the $P^{z}$ dependence.
There is actually one more requirement for the matching: there is a rapidity scheme dependence which must be removed, since the quasi-TMDPDF can be viewed as defined with an off-light-cone regulator along the $z$ direction. To understand this dependence, let us consider $f$ again in the off-light-cone regularization, where there are rapidity divergences. The divergence is cancelled by the square root of an off-light-cone soft function $S_{\mathrm{DY}}\left(b_{\perp}, \mu, Y, Y^{\prime}\right)$, with $Y, Y^{\prime}$ being the rapidities of the off-light-cone space-like vectors $p \rightarrow p_{Y}=p-$ $e^{-2 Y}\left(p^{+}\right)^{2} n$ and $n \rightarrow n_{Y^{\prime}}=n-e^{-2 Y^{\prime}} p /\left(p^{+}\right)^{2}$. Schematically, we have:

$$
S_{\mathrm{DY}}\left(b_{\perp}, \mu, Y, Y^{\prime}\right)=\frac{\operatorname{tr}\left\langle 0\left|\mathcal{W}_{n_{Y^{\prime}}}\left(\vec{b}_{\perp}\right) \mathcal{W}_{p_{Y}}^{\dagger}\left(\vec{b}_{\perp}\right)\right| 0\right\rangle}{N_{c} \sqrt{Z_{E}} \sqrt{Z_{E}}},
$$

where $\mathcal{W}_{n_{Y^{\prime}}}\left(\vec{b}_{\perp}\right)$ and $\mathcal{W}_{p_{Y}}^{\dagger}\left(\vec{b}_{\perp}\right)$ are staple-shaped gaugelinks in $n_{Y^{\prime}}, p_{Y}$ directions, respectively. $\sqrt{Z_{E}}$ is introduced to subtract the pinch pole singularities for the off-light-cone staple-shaped gauge-links. In terms of $\ln \rho^{2}=2\left(Y+Y^{\prime}\right)$ sometimes we also write this soft function as $S_{\mathrm{DY}}\left(b_{\perp}, \mu, \rho\right)$. At large $\rho$, we have

$$
S_{\mathrm{DY}}\left(b_{\perp}, \mu, Y, Y^{\prime}\right)=e^{\left(Y+Y^{\prime}\right) K\left(b_{\perp}, \mu\right)+\mathcal{D}\left(b_{\perp}, \mu\right)}+\ldots .
$$

We can perform a Lorentz boost of $\mathcal{W}_{n_{Y^{\prime}}}\left(\vec{b}_{\perp}\right) \mathcal{W}_{p_{Y}}^{\dagger}\left(\vec{b}_{\perp}\right)$ in Eq. (202) such that one of the gauge-links, say $\mathcal{W}_{n_{Y^{\prime}}}$, is boosted to the equal-time version $\mathcal{W}_{z}$ in $\tilde{f}$, whereas the other gauge-link $\mathcal{W}_{n_{Y}}$ is boosted to $\mathcal{W}_{n_{Y+Y^{\prime}}}$. The soft function becomes $S_{\mathrm{DY}}\left(b_{\perp}, \mu, Y+Y^{\prime}, 0\right)$ which contains light-cone divergence for the $p_{Y+Y^{\prime}}$ direction, but is still the same $S_{\mathrm{DY}}\left(b_{\perp}, \mu, Y, Y^{\prime}\right)$ due to boost invariance. The square-root of the finite part $e^{\mathcal{D}\left(b_{\perp}, \mu\right)}$ is exactly what is needed to cancel the rapidity-scheme dependence. We define the rapidity-independent part as the reduced soft function:

$$
S_{r}\left(b_{\perp}, \mu\right) \equiv e^{-\mathcal{D}\left(b_{\perp}, \mu\right)} .
$$

Based on the renormalization property of non-light-like Wilson-loops, the reduced soft function satisfies the RG equation

$$
\mu^{2} \frac{d}{d \mu^{2}} \ln S_{r}\left(b_{\perp}, \mu\right)=\Gamma_{S}\left(\alpha_{s}\right),
$$

where $\Gamma_{S}$ is the constant part of the cusp-anomalous dimension at large hyperbolic cusp angle $Y+Y^{\prime}$ for the off-light-cone soft function:

$$
\begin{aligned}
\mu^{2} \frac{d}{d \mu^{2}} \ln & S_{\mathrm{DY}}\left(b_{\perp}, \mu, Y, Y^{\prime}\right) \\
& =-\left(Y+Y^{\prime}\right) \Gamma_{\text {cusp }}\left(\alpha_{s}\right)-\Gamma_{S}\left(\alpha_{s}\right) .
\end{aligned}
$$

At one-loop level (Ebert et al., 2019b),

$$
S_{\mathrm{DY}}^{(1)}\left(b_{\perp}, \mu, Y, Y^{\prime}\right)=\frac{\alpha_{s} C_{F}}{2 \pi}\left[2-2\left(Y+Y^{\prime}\right)\right] L_{b},
$$


and $\Gamma_{S}^{(1)}\left(\alpha_{s}\right)=\alpha_{s} C_{F} / \pi$. Based on RGE, at two-loop level $\mathcal{D}\left(b_{\perp}, \mu\right)$ can be predicted to be

$$
\mathcal{D}^{(2)}\left(b_{\perp}, \mu\right)=c_{2}+\Gamma_{S}^{(2)} L_{b}-\frac{\alpha_{s}^{2} \beta_{0} C_{F}}{2 \pi} L_{b}^{2},
$$

where

$$
\Gamma_{S}^{(2)}=-\frac{\alpha_{s}^{2}}{\pi^{2}}\left[C_{F} C_{A}\left(-\frac{49}{36}+\frac{\pi^{2}}{12}-\frac{\zeta_{3}}{2}\right)+C_{F} N_{F} \frac{5}{18}\right]
$$

is the two-loop anomalous dimension for $S_{r}$ which can be extracted from (Grozin et al., 2016), $\beta_{0}=$ $-\left(\frac{11}{3} C_{A}-\frac{4}{3} N_{f} T_{F}\right) /(2 \pi)$ is the coefficient of one-loop $\beta$-function, and $c_{2}$ is a constant to be determined by explicit calculation.

After taking into account the reduced soft function, we can now write down the matching formula between the quasi-TMDPDF and the scheme-independent TMDPDF (Ji et al., 2019b):

$$
\begin{aligned}
& f^{\mathrm{TMD}}\left(x, b_{\perp}, \mu, \zeta\right) \\
& =H\left(\frac{\zeta_{z}}{\mu^{2}}\right) e^{-\ln \left(\frac{\zeta_{z}}{\zeta}\right) K\left(b_{\perp}, \mu\right)} \tilde{f}\left(x, b_{\perp}, \mu, \zeta_{z}\right) S_{r}^{\frac{1}{2}}\left(b_{\perp}, \mu\right)+\ldots
\end{aligned}
$$

where the power-corrections of order $\mathcal{O}\left(\Lambda_{\mathrm{QCD}}^{2} / \zeta_{z}, M^{2} /\left(P^{z}\right)^{2}, 1 /\left(b_{\perp}^{2} \zeta_{z}\right)\right)$. The above relation except for the definition of $S_{r}\left(b_{\perp}, \mu\right)$ was argued to hold in (Ebert et al., 2019b), where the unknown function $g_{q}^{S}$ in Eq. (5.3) should be identified as the reduced soft function here; it has also been confirmed recently in (Vladimirov and Schäfer, 2020).

We now explain the individual factors of the formula.

1. The factor $H\left(\zeta_{z} / \mu^{2}\right)$ is the perturbative matching kernel, which is a function of $\zeta_{z} / \mu^{2}=\left(2 x P^{z}\right)^{2} / \mu^{2}$. The kernel is responsible for the large logarithms of $P^{z}$ generated by the $\mathcal{G}\left(\zeta_{z} / \mu^{2}\right)$ term of the momentum RG equation. Unlike the case of quasi-PDFs, the momentum fractions of the quasi-TMDPDF and the TMDPDF are the same. This is due to the fact that at leading power in $1 / \zeta_{z}$ expansion, the $k_{\perp}$ integral is naturally cut off by the transverse separation around $k_{\perp} \sim 1 / b_{\perp} \ll P^{z}$. Therefore, the momentum fraction can only be modified by collinear modes for which there are no distinction between $x=k^{z} / P^{z}$ and $x=k^{+} / P^{+}$. In comparison, for the $\vec{k}_{\perp}$ integrated quasi-PDF, the $k_{\perp} \geq P^{z}$ region leads to non-trivial $x$ dependence outside the physical region. This is also consistent with the fact that the momentum evolution equation for quasiTMDPDF is local in $x$ instead of being a convolution.

2. The factor $\exp \left[\ln \left(\frac{\zeta_{z}}{\zeta}\right) K\left(b_{\perp}, \mu\right)\right]$ is the part involving the Collins-Soper evolution kernel. From the momentum evolution equation, it is clear that at large $P^{z}$ there are logarithms of the form
$K\left(b_{\perp}, \mu\right) \ln \frac{\zeta_{z}}{\mu^{2}}$ with $\zeta_{z}$ being the natural CollinsSoper scale. Therefore, to match to the TMDPDF at arbitrary $\zeta$, a factor $\exp \left[\ln \left(\frac{\zeta_{z}}{\zeta}\right) K\left(b_{\perp}, \mu\right)\right]$ is required to compensate the difference. An important implication of this property is that one can obtain the Collins-Soper kernel $K\left(b_{\perp}, \mu\right)$ by constructing the ratio of quasi-TMDPDFs at two different momenta or $\zeta_{z}$ 's (Ebert et al., 2019a),

$\frac{\tilde{f}\left(x, b_{\perp}, \mu, \zeta_{z, 1}\right)}{\tilde{f}\left(x, b_{\perp}, \mu, \zeta_{z, 2}\right)}=\frac{H\left(\frac{\zeta_{z, 2}}{\mu^{2}}\right)}{H\left(\frac{\zeta_{z, 1}}{\mu^{2}}\right)}\left(\frac{\zeta_{z, 1}}{\zeta_{z, 2}}\right)^{K\left(b_{\perp}, \mu\right)}$.

Thus given the $\tilde{f}$ 's at the two rapidity scales, the Collins-Soper kernel $K\left(b_{\perp}\right)$ can be obtained.

Combining the RGEs of the quasi-TMDPDF $\tilde{f}$, reduced soft function $S_{r}$ and physical TMDPDF $f^{\mathrm{TMD}}$, we obtain the RGE of the matching kernel $H\left(\frac{\zeta_{z}}{\mu^{2}}\right)$ (Ji et al., 2019b),

$$
\mu^{2} \frac{d}{d \mu^{2}} \ln H^{-1}\left(\frac{\zeta_{z}}{\mu^{2}}\right)=\frac{1}{2} \Gamma_{\text {cusp }}\left(\alpha_{s}\right) \ln \frac{\zeta_{z}}{\mu^{2}}+\frac{\gamma_{C}\left(\alpha_{s}\right)}{2},
$$

where $\gamma_{C}\left(\alpha_{s}\right)=2 \gamma_{F}\left(\alpha_{s}\right)+\Gamma_{S}\left(\alpha_{s}\right)+2 \gamma_{H}\left(\alpha_{s}\right)$. The matching kernel is closely related to the perturbative part of the rapidity evolution kernel $\mathcal{G}\left(\frac{\zeta_{z}}{\mu^{2}}\right)$ through

$$
2 \zeta_{z} \frac{d}{d \zeta_{z}} \ln H^{-1}\left(\frac{\zeta_{z}}{\mu^{2}}\right)=\mathcal{G}\left(\frac{\zeta_{z}}{\mu^{2}}\right) .
$$

Again, we can see that the anomalous dimension of $\mathcal{G}\left(\frac{\zeta_{z}}{\mu^{2}}\right)$ is $\Gamma_{\text {cusp }}\left(\alpha_{s}\right)$.

It is convenient to write $H$ in the exponential form, $H=e^{-h}$. Collecting all the above results, one obtains at one-loop level (Ebert et al., 2019b; Ji et al., 2019a)

$$
h^{(1)}\left(\frac{\zeta_{z}}{\mu^{2}}\right)=\frac{\alpha_{s} C_{F}}{2 \pi}\left(-2+\frac{\pi^{2}}{12}-\frac{L_{z}^{2}}{2}+L_{z}\right) .
$$

Similar as before, the two loop contribution $h^{(2)}$ is predicted to be

$$
\begin{aligned}
& h^{(2)}\left(\frac{\zeta_{z}}{\mu^{2}}\right)=c_{2}^{\prime}-\frac{1}{2}\left(\gamma_{C}^{(2)}-\alpha_{s}^{2} \beta_{0} c_{1}\right) \ln \frac{\zeta_{z}}{\mu^{2}} \\
& -\frac{1}{4}\left(\Gamma_{\text {cusp }}^{(2)}-\frac{\alpha_{s}^{2} \beta_{0} C_{F}}{2 \pi}\right) \ln ^{2} \frac{\zeta_{z}}{\mu^{2}}-\frac{\alpha_{s}^{2} \beta_{0} C_{F}}{24 \pi} \ln ^{3} \frac{\zeta_{z}}{\mu^{2}},
\end{aligned}
$$

where $c_{1}=\frac{C_{F}}{2 \pi}\left(-2+\frac{\pi^{2}}{12}\right)$ and $c_{2}^{\prime}$ is again a constant to be determined in perturbation theory at two-loop level.

Finally, we compare the current formulation with previous approaches to lattice TMDPDF. First, we comment on the developments in (Engelhardt et al., 2016; Hagler et al., 2009; Musch et al., 2012, 2011; Yoon et al., 2017) in which the $x$-moments of TMDPDF are extracted 
from ratio of quasi-TMDPDF. From Eq. (209), it is clear that both the matching kernel $H$ and the exponential factor of Collins-Soper kernel depends on $x$ nontrivially. Therefore, simply taking the ratio of moments for quasi-TMDPDF will not be sufficient to reproduce the same ratio for TMDPDF, although the soft function does cancel. This observation is also made recently in Ref. (Ebert et al., 2020a). Second, the quasi-TMDPDF defined with the naive rectangle-shaped soft function, i.e. $Z_{E}$, is $\tilde{f}$ in Eq. (195), so it is obvious that it still needs the reduced soft function $S_{r}$ to be matched to $f^{\mathrm{TMD}}$. As for the other proposal in (Ebert et al., 2019b; Ji et al., $2015 \mathrm{~b})$, it replaces $Z_{E}$ in $\tilde{f}$ with $S_{\text {bent }}$ which is the vacuum matrix element of a spacelike bent-shaped Wilson loop with angle $\pi / 2$ at each junction, and does not include the function $S_{r}^{\frac{1}{2}}$ in Eq. (209). Although $\sqrt{S_{\text {bent }} / Z_{E}}$ agrees with $S_{r}^{\frac{-1}{2}}$ at one-loop order (Ebert et al., 2019b; Ji et al., 2019a), it is expected to be different at higher orders. In fact, for the anomalous dimension $\Gamma_{\frac{\pi}{2}}$ defined through

$$
\Gamma_{\frac{\pi}{2}}\left(\alpha_{s}\right) \equiv \mu^{2} \frac{d}{d \mu^{2}} \ln \left(\frac{S_{\text {bent }}\left(L, b_{\perp}, \mu\right)}{Z_{E}\left(2 L, b_{\perp}, \mu\right)}\right),
$$

it starts to deviate from $\Gamma_{S}\left(\alpha_{s}\right)$ at two-loop order (Grozin et al., 2016), as

$$
\begin{aligned}
-\Gamma_{S}\left(\alpha_{s}\right) & =\frac{\alpha_{s} C_{F}}{\pi} \\
& +\frac{\alpha_{s}^{2}}{\pi^{2}}\left[C_{F} C_{A}\left(-\frac{49}{36}+\frac{\pi^{2}}{12}-\frac{\zeta_{3}}{2}\right)+C_{F} N_{F} \frac{5}{18}\right], \\
\Gamma_{\frac{\pi}{2}}\left(\alpha_{s}\right) & =\frac{\alpha_{s} C_{F}}{\pi} \\
& +\frac{\alpha_{s}^{2}}{\pi^{2}}\left[C_{F} C_{A}\left(-\frac{49}{36}+\frac{\pi^{2}}{24}\right)+C_{F} N_{F} \frac{5}{18}\right] .
\end{aligned}
$$

In the equation, $\zeta_{3}=\sum_{n=1}^{\infty}\left(1 / n^{3}\right) \neq \pi^{2} / 12$, therefore the two anomalous dimensions are different. The differences in the anomalous dimension will result in different logarithmic behaviors in $b_{\perp}$, as the soft functions are dimensionless and depend on $b_{\perp}$ and $\mu$ only. At large $b_{\perp}$, it will lead to different IR physics that cannot be controlled by perturbation theory.

Combining the reduced soft function and the quasiTMDPDF, one can effectively factorize the DY cross section,

$$
\begin{aligned}
& \sigma=\int d x_{A} d x_{B} d^{2} b_{\perp} e^{i \vec{Q}_{\perp} \cdot \vec{b}_{\perp}} \hat{\sigma}\left(x_{A}, x_{B}, Q^{2}, \mu\right) \\
& \times \tilde{f}\left(x_{A}, b_{\perp}, \mu, \zeta_{A}\right) \tilde{f}\left(x_{B}, b_{\perp}, \mu, \zeta_{B}\right) S_{r}\left(b_{\perp}, \mu\right) .
\end{aligned}
$$

where all non-perturbative quantities do not involve the light cone, and can be calculated on lattice.

Spin-dependent TMDPDFs are also physically important. They can be computed using LaMET theory (Ebert et al., 2020a). Again one can define quasi distributions just like the spin-independent ones. For a general proton target $|P S\rangle$ and the general spin structure $\Gamma$ of the parton, the parent TMDPDF can be defined as

$$
\begin{aligned}
& f_{[\Gamma]}^{\mathrm{TMD}}\left(x, \vec{k}_{\perp}, \mu, \zeta\right)=\frac{1}{2 P^{+}} \int \frac{d \lambda}{2 \pi} \int \frac{d^{2} \vec{b}_{\perp}}{(2 \pi)^{2}} e^{-i \lambda x+i \vec{k}_{\perp} \cdot \vec{b}_{\perp}} \\
& \times \lim _{\delta^{-} \rightarrow 0} \frac{\left\langle P S\left|\bar{\psi}\left(\lambda n+\vec{b}_{\perp}\right) \Gamma \mathcal{W}_{n}\left(\lambda n+\vec{b}_{\perp}\right)\right|_{\delta^{-}} \psi(0) \mid P S\right\rangle}{\sqrt{S\left(b_{\perp}, \mu, \delta^{-} e^{2 y_{n}}, \delta^{-}\right)}},
\end{aligned}
$$

where the $\zeta=2\left(P^{+}\right)^{2} e^{2 y_{n}}$ is the rapidity scale, see Sec. $\mathrm{V}$ for more detail of the soft function subtraction. The individual spin-dependent TMD distributions can then be obtained through Lorentz decompositions (Mulders and Tangerman, 1996; Ralston and Soper, 1979; Tangerman and Mulders, 1995):

$$
\begin{aligned}
f_{\left[\gamma^{+}\right]}^{\mathrm{TMD}}= & f_{1}-\frac{\epsilon^{i j} k^{i} S_{\perp}^{j}}{M} f_{1 T}^{\perp}, \\
f_{\left[\gamma^{+} \gamma_{5}\right]}^{\mathrm{TMD}}= & S^{+} g_{1}+\frac{\vec{k}_{\perp} \cdot \vec{S}_{\perp}}{M} g_{1 T}, \\
f_{\left[i \sigma^{i+} \gamma_{5}\right]}^{\mathrm{TMD}}= & S_{\perp}^{i} h_{1}+\frac{\left(2 k^{i} k^{j}-\vec{k}_{\perp}^{2} \delta^{i j}\right) S_{\perp}^{j}}{2 M^{2}} h_{1 T}^{\perp} \\
& +\frac{S^{+} k^{i}}{M} h_{1 L}^{\perp}+\frac{\epsilon^{i j} k^{j}}{M} h_{1}^{\perp},
\end{aligned}
$$

where we suppress the arguments $\left(x, \vec{k}_{\perp}, \mu, \zeta\right)$ in all distributions; $f_{1}, g_{1}$, and $h_{1}$ are unpolarized, helicity and transversity TMDPDFs, respectively; the indices $i$ and $j$ are in transverse space of $\vec{k}_{\perp} ; S^{+}$and $S_{\perp}^{i}$ are longitudinal and transverse spin components.

Note that the Sivers function $f_{1 T}^{\perp}$ (Sivers, 1990) and the Boer-Mulders function $h_{1}^{\perp}$ (Boer and Mulders, 1998) are T-odd. The orientation of the gauge-link have important effects on these two functions Collins, 2011a, 2002), such that they change sign between the DY and SIDIS processes. In the light-cone gauge, these contributions arise from the transversal gauge-link at infinities (Belitsky et al., 2003). They are related to the phenomenologically interesting single transversespin asymmetry (Boer and Mulders, 1998; Collins et al., 2005, 2006; Efremov et al., 2005).

\section{Off-light-cone Soft Function}

In previous subsections, the soft function has been introduced to define rapidity-scheme-independent TMDPDFs. The major motivation of introducing the soft function is to capture nonperturbative effects due to softgluon radiations from fast moving color-charges. For many inclusive processes the soft radiations cancel in the total cross section, but for certain processes where a small transverse momentum is measured, such cancellation can be incomplete and result in measurable con- 
sequences. In such cases, the TMD soft function is introduced to account for the soft-gluon effects and appears in factorization theorems for the Drell-Yan (DY) process (Collins et al., 1985b, 1988) and semi-inclusive DIS (SIDIS) (Ji et al., 2004, 2005).

To calculate the TMD physics nonperturbatively, formulating a Euclidean version of the soft function is critical. Since the soft function in fact is a cross section and hence real and positive, it satisfies the necessary condition for a Monte Carlo simulation. In this subsection, we present an approach to calculate it in heavy-quark effective theory (HQET) (Ji et al., 2020a). There is also another method proposed to extract the reduced soft function $S_{r}$ from a light-meson form factor (Ji et al., 2020a), where many subtleties of HQET can be avoided. The first lattice calculation of the reduced soft function based on the light-meson formalism has been performed in the recent work (Zhang et al., 2020a).

Due to the different space-time pictures of the DY and SIDIS processes, the soft functions for the two processes also differ from each other as shown in Fig. 11. To define the soft function, one also needs to specify a timeordering prescription. Since it is a cross section, it involves a time order and an anti-time order (or cut diagrams). However, in the light-cone limit, the time order does not matter. What really matters is the rapidity regularization scheme. It has been proven for the $\delta$ regulator in (Vladimirov, 2018) that the time ordering is not quite relevant up to overall phase factors, and the soft functions for the two processes are equal. The method therein can be modified to apply to the off-light-cone scheme too. Therefore, our first step is to convert the cut-diagrams into Feynman diagrams by imposing just the single time order. In this way, the soft function can be viewed as a scattering amplitude.

In the off-light-cone scheme, there are further complications caused by the space-like or time-like choices for off-light-cone vectors. Fortunately, one can show that in the light-cone limit, the space-like and timelike choices are equivalent up to overall phase factors (Ji et al., in preparation). Thus we will use the notation $S\left(b_{\perp}, \mu, Y, Y^{\prime}\right)$ to denote a generic off-light-cone soft function that satisfies our demands.

With these in mind, we show that the off-light-cone soft function $S\left(b_{\perp}, \mu, Y, Y^{\prime}\right)$ is equivalent to an equaltime form factor of fast-moving color sources and can be formulated on the Euclidean lattice. From the matching formula Eq. (209) in the last subsection, once the off-light-cone soft function is known, we can combine it with the lattice calculated quasi-TMDPDF to obtain the physical TMDPDF. Therefore, the cross section of DY processes in the low transverse-momentum region (Collins et al., 1985b) becomes predictable from first principles (Ji et al., 2020a).

To begin with, we define the scattering amplitude of a
Wilson loop as shown in Fig. 13.

$$
\begin{aligned}
& W\left(t, t^{\prime}, b_{\perp}, Y, Y^{\prime}\right) \\
& =\frac{1}{N_{c}} \operatorname{Tr}\left\langle 0\left|\mathcal{T}\left[\mathcal{W}_{v^{\prime}}^{\dagger}\left(\vec{b}_{\perp}, t^{\prime}\right) \mathcal{W}_{v}\left(\vec{b}_{\perp}, t\right)\right]\right| 0\right\rangle
\end{aligned}
$$

where $|0\rangle$ is the QCD vacuum state and $N_{c}$ is number of colors and $\mathrm{Tr}$ is the color-trace. Timelike fourvectors $v^{\mu}=\gamma\left(1, \beta, \overrightarrow{0}_{\perp}\right)$ and $v^{\prime \mu}=\gamma^{\prime}\left(1,-\beta^{\prime}, \overrightarrow{0}_{\perp}\right)$ approach the lightcone as $\beta$ and $\beta^{\prime} \rightarrow 1$. The rapidity $Y$ and the speed $\beta$ are related through $\beta=\tanh Y$, in terms of the light-cone vectors $p$ and $n$, the velocities read $v=\frac{e^{Y}}{\sqrt{2}}\left(\frac{p}{p^{+}}+e^{-2 Y} p^{+} n\right)$ and $v^{\prime}=\frac{e^{Y}}{\sqrt{2}}\left(e^{-2 Y} \frac{p}{p^{+}}+p^{+} n\right)$. The $\mathcal{W}_{v}\left(\vec{b}_{\perp}, t\right)$ is a staple-shaped gauge-link along $v$ direction similar to those defined in Eqs. (173) and (196). $t$ and $t^{\prime}$ are the lengths of the $t$-components of the staples. The single time-order prescription for $S$ allows physical interpretation as a chronological process. Similar to the

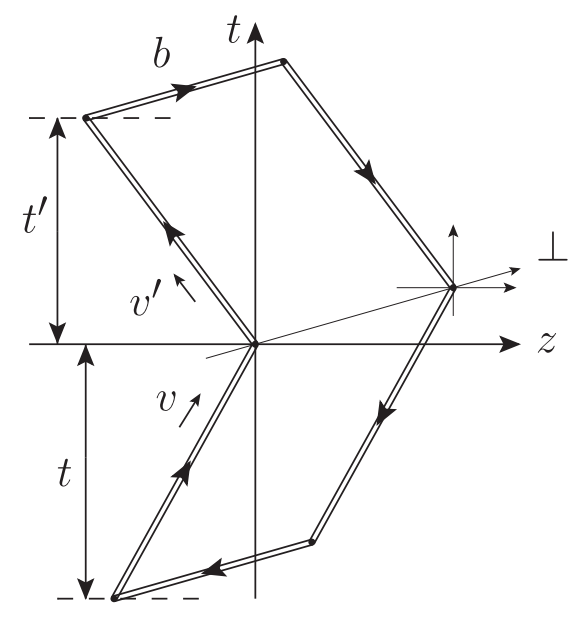

FIG. 13: The Wilson-loop $\mathcal{W}$ showing a pair of quark and antiquark scattering at $t=0$.

quasi-TMDPDF, the Wilson-loop in Eq. (223) contains pinch-pole singularities associated to time evolution of initial and final states at large $t$ and $t^{\prime}$. Therefore, we need to subtract them out in Eq. (223) with rectangular Wilson-loops (Collins, 2008; Ji et al., 2019a). This leads to an off-light-cone realization of the soft function:

$$
\begin{aligned}
& S\left(b_{\perp}, \mu, Y, Y^{\prime}\right) \\
& =\lim _{\substack{t \rightarrow \infty \\
t^{\prime} \rightarrow \infty}} \frac{W\left(t, t^{\prime}, b_{\perp}, \mu, Y, Y^{\prime}\right)}{\sqrt{Z\left(2 t, b_{\perp}, \mu, Y\right) Z\left(2 t^{\prime}, b_{\perp}, \mu, Y^{\prime}\right)}},
\end{aligned}
$$

where $Z\left(2 t, b_{\perp}, Y\right)$ is the vacuum expectation value of rectangular Wilson loop which is similar to $W$ by setting $v^{\prime}=v$ and $t^{\prime}=t$, i.e. $Z\left(2 t, b_{\perp}, Y\right)=W\left(t, t, b_{\perp}, Y,-Y\right)$. The factor $Z$ has a clear physical interpretation: It can be viewed as the wave function renormalization for incoming or outgoing color sources. After the subtraction through $Z$, the only remaining UV divergences for $S\left(b_{\perp}, \mu, Y, Y^{\prime}\right)$ are the cusp divergences with hyperbolic angle $Y+Y^{\prime}$. 
We should mention that a more common definition of the soft function $S_{\mathrm{DY}}\left(b_{\perp}, \mu, Y, Y^{\prime}\right)$ for the DY process was proposed in (Collins, 2011a, b). The space-like vectors $u^{\mu}=\gamma(\beta, 1,0,0)$ and $u^{\prime \mu}=\gamma^{\prime}\left(-\beta^{\prime}, 1,0,0\right)$ were chosen instead of time-like $v$ and $v^{\prime}$ to define the soft function for the DY process. This soft function has already been defined in the last subsection in Eq. (202). $u$ and $u^{\prime}$ are equal to $p_{Y}, n_{Y^{\prime}}$ up to overall normalization factors.

While $S$ and $S_{\mathrm{DY}}$ are defined differently, we can show that

$$
S\left(b_{\perp}, \mu, Y, Y^{\prime}\right)=S_{\mathrm{DY}}\left(b_{\perp}, \mu, Y, Y^{\prime}\right)
$$

using analyticity property (Ji et al., in preparation). Here we focus on $S$ in Eq. (224), which has a simple Euclidean realization.

After defining the soft function $S$, we now show that it is equal to a form factor. In HQET, the propagator of a color source is equivalent to a gauge-link along its moving direction. Thus $W\left(t, t^{\prime}, b_{\perp}, \mu, Y, Y^{\prime}\right)$ can be expressed by fields in HQET with the Lagrangian

$$
\mathcal{L}_{\mathrm{HQET}}=Q_{v}^{\dagger}(x)(i v \cdot D) Q_{v}(x)+\bar{Q}_{v}^{\dagger}(x)(i v \cdot D) \bar{Q}_{v}(x),
$$

where $Q_{v}$ and $\bar{Q}_{v}$ are quark and anti-quark in the fundamental and anti-fundamental representations, respectively; $v^{\mu}=\gamma\left(1, \beta, \overrightarrow{0}_{\perp}\right)$ is the four velocity; $D$ is the covariant derivative. Note that quarks in HQET can be viewed as color sources. If the gluon soft function is considered, the heavy quarks should be in the adjoint representation.

In HQET, a color-singlet heavy-quark pair separated by $\vec{b}$ generates a heavy quark potential $V(|\vec{b}|)$ in the ground state, and the spectrum includes a gapped continuum above it. The state can also have a residual momentum $\delta \vec{P}$, which is arbitrary due to reparameterization invariance (Luke and Manohar, 1992; Manohar and Wise, 2000), and for simplicity we always consider $\delta \vec{P}=0$. When the sources move with a velocity $v$, the ground state can be labeled by $|\bar{Q} Q, \vec{b}, \delta \vec{P}\rangle_{v}$, where the residue momentum $\delta \vec{P}=\vec{P}_{\text {total }}-2 m_{Q} \gamma \vec{\beta}$ is the difference between the total momentum $\vec{P}_{\text {total }}$ and the kinetic momentum of the heavy-quarks. The residual energy of the state is $E=\gamma^{-1} V\left(\left|\vec{b}_{\perp}\right|\right)+\vec{\beta} \cdot \delta \vec{P}$.

Consider a process with incoming and outgoing states being heavy-quark pairs separated by $\vec{b}_{\perp}$ and at velocity $v$ and $v^{\prime}$, respectively. Such a state is created by the interpolating fields

$$
\mathcal{O}_{v}\left(t, \vec{b}_{\perp}\right)=\int d^{3} \vec{r} Q_{v}^{\dagger}(t, \vec{r}) \mathcal{U}\left(\vec{r}, \vec{r}^{\prime}, t\right) \bar{Q}_{v}^{\dagger}\left(t, \vec{r}^{\prime}\right),
$$

where $\vec{r}^{\prime}=\vec{r}+\vec{b}_{\perp}$ and $\mathcal{U}\left(\vec{r}, \vec{r}^{\prime}, t\right)$ is a gauge-link connecting $\vec{r}^{\prime}$ to $\vec{r}$ at time $t$. The heavy-quark pair created by $\mathcal{O}_{v}$ is forced to be at relative separation $\vec{b}_{\perp}$ and to have vanishing residual momentum $\delta \vec{P}=0$. Between the incoming and outgoing states, a product of two local equal-time operators

$$
J\left(v, v^{\prime}, \vec{b}_{\perp}\right)=\bar{Q}_{v^{\prime}}^{\dagger}\left(\vec{b}_{\perp}\right) \bar{Q}_{v}\left(\vec{b}_{\perp}\right) Q_{v^{\prime}}^{\dagger}(0) Q_{v}(0)
$$

is inserted at $t=0$. Then $W$ can be expressed in terms of HQET propagators which are gauge-links in the $v, v^{\prime}$ directions. After integrating out the heavy-quark fields, we obtain up to an overall volume factor (Ji et al., 2020a)

$$
\begin{aligned}
& W\left(t, t^{\prime}, b_{\perp}, \mu, Y, Y^{\prime}\right) \\
& =\frac{1}{N_{c}}\left\langle 0\left|\mathcal{O}_{v^{\prime}}^{\dagger}\left(t^{\prime}, \vec{b}_{\perp}\right) J\left(v, v^{\prime}, \vec{b}_{\perp}\right) \mathcal{O}_{v}\left(-t, \vec{b}_{\perp}\right)\right| 0\right\rangle \\
& \underset{\substack{t \rightarrow \infty \\
t^{\prime} \rightarrow \infty}}{\longrightarrow} \frac{1}{N_{c}} \Phi^{\dagger}\left(b_{\perp}, \mu\right) S\left(b_{\perp}, \mu, Y, Y^{\prime}\right) \Phi\left(b_{\perp}, \mu\right) e^{-i E^{\prime} t^{\prime}-i E t},
\end{aligned}
$$

where

$$
\begin{aligned}
\Phi\left(b_{\perp}, \mu\right) & =\lim _{T \rightarrow \infty}\left\langle\bar{Q} Q, \vec{b}_{\perp}\left|\mathcal{O}_{v}\left(T, \vec{b}_{\perp}\right)\right| 0\right\rangle, \quad \\
S\left(b_{\perp}, \mu, Y, Y^{\prime}\right) & ={ }_{v^{\prime}}\left\langle\bar{Q} Q, \vec{b}_{\perp}\left|J\left(v, v^{\prime}, \vec{b}_{\perp}\right)\right| \bar{Q} Q, \vec{b}_{\perp}\right\rangle_{v} .
\end{aligned}
$$

In the last line of Eq. (229), we have inserted a complete set of heavy-quark pair states before and after $J$. At large $t$ and $t^{\prime}$, the contribution from the continuum spectrum is damped out due to the RiemannLebesgue lemma (Zuazo, 2001), while the contribution from $\left|\bar{Q} Q, \vec{b}_{\perp}, \delta \vec{P}=0\right\rangle_{v}$ with residual energy $E=$ $\gamma^{-1} V\left(\left|\vec{b}_{\perp}\right|\right)$ survives. As a result we obtain Eqs. (229)(230), where we have omitted the state label $\delta \vec{P}=0$ for simplicity. Alternatively, we can also give $t$ and $t^{\prime}$ a small negative imaginary part, which is consistent with the time order, to damp out all states except $\left|\bar{Q} Q, \vec{b}_{\perp}\right\rangle_{v}$ at large $t$ and $t^{\prime}$. Note that $\Phi\left(\vec{b}_{\perp}, \mu\right)$ is independent of $Y$ because it is boost invariant.

Similarly, $Z$ can also be formulated in HQET as

$$
\begin{aligned}
Z\left(2 t, b_{\perp}, Y\right) & =\frac{1}{N_{c}}\left\langle 0\left|\mathcal{O}_{v^{\prime}}^{\dagger}\left(t, \vec{b}_{\perp}\right) \mathcal{O}_{v}\left(-t, \vec{b}_{\perp}\right)\right| 0\right\rangle \\
& \underset{t \rightarrow \infty}{\longrightarrow} \frac{1}{N_{c}} \Phi^{\dagger}\left(\vec{b}_{\perp}, \mu\right) \Phi\left(\vec{b}_{\perp}, \mu\right) e^{-2 i E t},
\end{aligned}
$$

whose $t$-component has length $2 t$. The $Y$ dependence of $Z$ is implicit in the energy $E$. Combining Eqs. (229) and (231), we obtain $S$ defined in Eq. (224). We emphasize that Eq. (224) can be seen as a LSZ reduction formula, in which we amputate the external heavy-quark pair states $\left|\bar{Q} Q, \vec{b}_{\perp}\right\rangle_{v}$.

Being an equal-time observable, $S\left(b_{\perp}, \mu, Y, Y^{\prime}\right)$ can be straightforwardly realized in Euclidean time as:

$$
\begin{aligned}
& S\left(b_{\perp}, \mu, Y, Y^{\prime}\right) \\
& =\lim _{\substack{T \rightarrow \infty \\
T^{\prime} \rightarrow \infty}} \frac{W_{E}\left(T, T^{\prime}, b_{\perp}, \mu, Y, Y^{\prime}\right)}{\sqrt{Z_{E}\left(2 T, b_{\perp}, \mu, Y\right) Z_{E}\left(2 T^{\prime}, b_{\perp}, \mu, Y^{\prime}\right)}},
\end{aligned}
$$

where the subscript $E$ indicates the quantity is defined in Euclidean time, with corresponding variables 
$T$ and $T^{\prime}$. Due to boost invariance, the factor $Z_{E}\left(T, b_{\perp}, \mu, Y\right)$ relates to the rectangular Wilson-loop defined in Eq. (198) along the $n_{z}$ direction through the relation $Z_{E}\left(2 T, b_{\perp}, \mu, Y\right)=Z_{E}\left(2 \gamma^{-1} T, b_{\perp}, 0\right)$. The relevant matrix elements are now calculated by a lattice version of HQET with the Lagrangian (Aglietti, 1994; Hashimoto and Matsufuru, 1996; Horgan et al., 2009)

$$
\begin{aligned}
& \mathcal{L}_{\mathrm{HQET}}^{E} \\
& =Q_{v}^{\dagger}(x)\left(i \tilde{v} \cdot D_{E}\right) Q_{v}(x)+\bar{Q}_{v}^{\dagger}(x)\left(i \tilde{v} \cdot D_{E}\right) \bar{Q}_{v}(x),
\end{aligned}
$$

where the subscript $E$ denotes the Euclidean space, $i \tilde{v} \cdot D_{E}=\gamma\left(D^{\tau}-i \beta\right) D^{z}$ with $\tilde{v}^{\mu}=\gamma\left(-i,-\beta, \overrightarrow{0}_{\perp}\right)$. We have explicitly verified Eq. (232) in Euclidean perturbation theory to the one-loop order.

The soft function cannot be calculated on the lattice by simply replacing the Minkowskian gauge-links in Eq. (223) with a finite number of Euclidean gauge-links. Through HQET, we find a time-independent formulation of the soft function, which opens up the possibility of direct lattice calculations.

\section{Light-Front Wave-Function Amplitudes And Soft Function from Meson Form Factor}

Light-front quantization (LFQ) or formalism is a natural language for parton physics in which partons are made manifest at all stages of calculations. It favors a Hamiltonian approach to QCD like for a non-relativistic quantum mechanical system, i.e., to diagonalize the Hamiltonian

$$
\hat{P}^{-}\left|\Psi_{n}\right\rangle=\frac{M_{n}^{2}+\vec{P}_{\perp}^{2}}{2 P^{+}}\left|\Psi_{n}\right\rangle
$$

to obtain wave functions for the QCD bound states (Brodsky et al., 1998). The light-front wave functions (LFWFs) thus obtained can, in principle, be used to calculate all the partonic densities and correlations functions. Moreover, like in condensed matter systems, knowing quantum many-body wave-functions allows one to understand interesting aspects of quantum coherence and entanglement, as well as the fundamental nature of quantum systems. Therefore, a practical realization of light-front quantization program clearly would be a big step forward in understanding the fundamental structure of the proton.

However, from a field theory point of view, wave functions are not the most natural objects to consider due to the non-trivial vacuum, UV divergences as well as the requirement of Lorentz symmetry, according to which the space and time should be treated on equal footing. The proton or other hadrons are excitations of the QCD vacuum which by itself is very complicated because of the well-known phenomena of chiral symmetry breaking and color confinement. To build a proton on top of this vacuum, one naturally has a question of what part of the wave-function reflects the property of the proton and what reflects the vacuum: It is the difference that yields the properties of the proton that are experimentally measurable. There is no clean way to make this separation unless one builds the proton out of elementary excitations or quasi-particles that do not exist in the vacuum, as often done in condensed matter systems.

The partons in the IMF avoids the above problems to a certain extent. In fact, due to the kinematic effects, in the IMF all partons in the vacuum have longitudinal momentum $k^{+}=0$, and to some degree of accuracy, the proton is made of partons with $k^{+} \neq 0$. This natural separation of degrees of freedom (DOF) is particularly welcome, making a wave-function description of the proton more natural and interesting in IMF than in any other frame.

To implement the above DOF separation, one possibility is to assume triviality of the light-front vacuum. The question that to what extent this holds has been continuously debated over the years. One knows a priori that in relativistic QFT, the vacuum state is boost invariant and frame-independent. In fact, it was proven in Nakanishi and Yabuki, 1977; Nakanishi and Yamawaki, 1977) that not only the vacuum can not be trivial, even the Green's functions of the full theory cannot pose generic meaningful restrictions to the null-planes $\xi^{+}=c$. In fact, the vacuum zero modes do contain non-trivial dynamics and contribute to the properties of the proton ( $\mathrm{Ji}, 2020)$. Nevertheless, one can adopt an effective theory point of view to simply cut off the zero-modes and relegate their physics to renormalization constants. In some simple cases, these zero-modes can be treated explicitly (Heinzl et al., 1991; Yamawaki, 1998).

By imposing an IR cut-off on the $k^{+} \geq \epsilon$ in the effective Hilbert space, all physics below $k^{+}=\epsilon$ are taken into account through renormalization constants. We then obtain an effective LF theory with trivial vacuum,

$$
a_{k \lambda}|0\rangle=b_{p \sigma}|0\rangle=d_{p \sigma}|0\rangle=0 .
$$

where $|0\rangle$ is the vacuum of LFQ. Therefore, the proton can be expanded in terms of the superposition of Fock states in the LF gauge $A^{+}=0$ (Brodsky et al. , 1998),

$$
|P\rangle=\sum_{n=1}^{\infty} \int d \Gamma_{n} \psi_{n}^{0}\left(x_{i}, \vec{k}_{i \perp}\right) \prod a_{i}^{\dagger}\left(x_{i}, \vec{k}_{i \perp}\right)|0\rangle .
$$

where $a^{\dagger}$ are generic quarks and gluon quanta on the light-front, the phase-space integral reads $d \Gamma_{n}=$ $\prod \frac{d k^{+} d^{2} k_{\perp}}{2 k^{+}(2 \pi)^{3}}$. The $\psi_{n}\left(x_{i}, \vec{k}_{i \perp}\right)$ are LFWF amplitudes or simply WF amplitudes, where $x_{i}$ to denote the set of momentum fractions from $x_{1}$ to $x_{n}$. They are a complete set of non-perturbative quantities which describe the partonic landscape of the proton. The above amplitudes can in principle be calculated through Hamiltonian 
diagonalization. However, as explained in Sec. II.A a direct systematic solution in LFQ is impractical.

LaMET offers an alternate route to calculate these WF amplitudes. Thanks to the triviality of the vacuum after the truncation $k^{+} \geq \epsilon$, they can then be written in terms of the invariant matrix elements by inverting the above expansion,

$$
\psi_{n}^{0}\left(x_{i}, \vec{k}_{i \perp}\right)=\left\langle 0\left|\prod a_{i}\left(x_{i}, \vec{k}_{i \perp}\right)\right| P\right\rangle .
$$

After properly restoring gauge-invariance and imposing regularizations, they become the matrix elements of lightcone correlators, the same type as those in the TMDPDFs. Therefore, the LaMET method applies to them, which allows one to effectively obtain the results of lightfront quantization through instant quantization in a large momentum frame.

To realize the goal, the LFWF amplitudes also need a rapidity renormalization, as in the case of TMDPDFs. In this section, we explain how the reduced soft function $S_{r}$ can be obtained by combining the LFWF amplitudes and a special light-meson form factor, instead of as the form factor in HQET discussed in the previous section. A lattice calculation based on the light-meson framework has been performed in (Zhang et al., 2020a).

Let us consider the following form factor of a pseudoscalar light-meson state with constituents $\bar{\psi} \eta$,

$$
F\left(b_{\perp}, P, P^{\prime}, \mu\right)=\left\langle P^{\prime}\left|\bar{\eta}\left(\vec{b}_{\perp}\right) \Gamma^{\prime} \eta\left(\vec{b}_{\perp}\right) \bar{\psi}(0) \Gamma \psi(0)\right| P\right\rangle
$$

where $\psi$ and $\eta$ are light quark fields of different flavors; $P^{\mu}=\left(P^{t}, 0,0, P^{z}\right)$ and $P^{\prime \mu}=\left(P^{t}, 0,0,-P^{z}\right)$ are two large momenta which approach two opposite light-like directions in the limit $P^{z} \rightarrow \infty ; \Gamma$ and $\Gamma^{\prime}$ are Dirac gamma matrices, which can be chosen as $\Gamma=\Gamma^{\prime}=1, \gamma_{5}$ or $\gamma_{\perp}$ and $\gamma_{\perp} \gamma_{5}$, so that the quark fields have leading components on the respective light-cones.

At large momentum, the form factor factorizes through TMD factorization into LFWF amplitudes. To motivate the factorization, we need to consider the leading region of IR divergences in a similar way for SIDIS and DrellYan (Collins, 2011b; Ji et al., 2005), and the result is shown in Fig. 14. There are two collinear sub-diagrams responsible for collinear modes in + and - directions, and a soft sub-diagram responsible for soft contributions. Besides, there are two IR-free hard cores localized around $(0,0,0,0)$ and $\left(0, \vec{b}_{\perp}, 0\right)$. In the covariant gauge, there are arbitrary numbers of longitudinally-polarized collinear and soft gluons that can connect to the hard and collinear sub-diagrams. Based on the region decomposition, we now follow the standard procedure to make factorization into LF quantities (Collins, 2011b).

We first factorize the soft divergences. This can be done with the soft function $S\left(b_{\perp}, \mu, \delta^{+}, \delta^{-}\right)$. It re-sums the soft gluon radiations from fast-moving color-charges. Intuitively, soft gluons have no impact on the velocity of

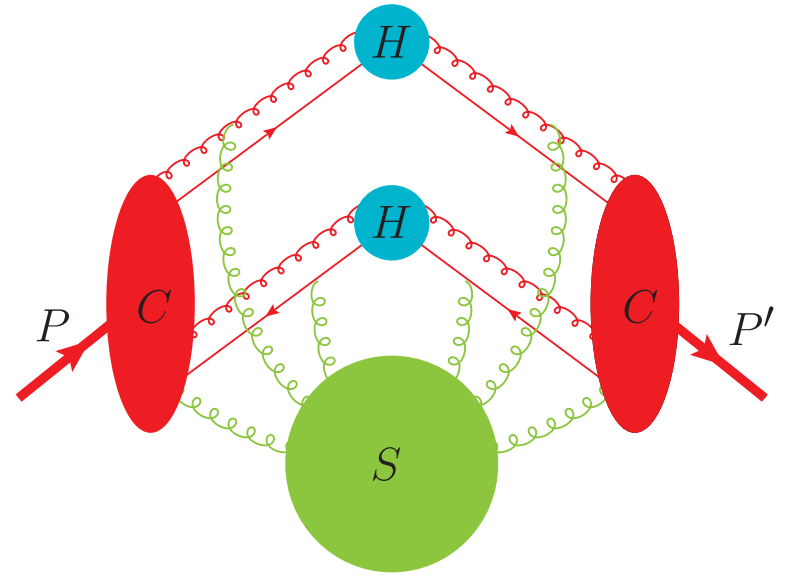

FIG. 14: The reduced diagram for the large-momentum form factor $F$ of a meson. Two $H$ denote the two hard cores separated in space by $\vec{b}_{\perp}, C$ are collinear sub-diagrams and $S$ denotes the soft sub-diagram.

the fast-moving color charged partons, and the propagators of partons eikonalize to straight gauge links along their moving trajectory.

We then factorize the collinear divergences. For the incoming direction, the collinear divergences is captured by the LFWF amplitude for the incoming parton $\psi_{\bar{q} q}\left(x, b_{\perp}, \mu, \delta^{\prime}-\right)$ defined with future-pointing gaugelinks.

$$
\begin{aligned}
& \psi_{\bar{q} q}\left(x, b_{\perp}, \mu, \delta^{\prime}-\right)=\int \frac{d \lambda}{4 \pi} e^{-i x \lambda} \\
& \left\langle 0\left|\bar{\psi}\left(\lambda n / 2+\vec{b}_{\perp}\right) \gamma^{+} \mathcal{W}_{n}\left(\lambda n / 2+\vec{b}_{\perp}\right)\right|_{\delta^{\prime}-} \psi(-\lambda n / 2) \mid P\right\rangle,
\end{aligned}
$$

where the staple-shaped gauge-link $W_{n}$ is defined similar to that in Eq. (166), the only exception being the gaugelinks $W_{n}$ should point to $+\infty$ instead of $-\infty$.

However, the naive LFWF amplitude contains soft divergences as well, to avoid double-counting, we must subtract out the soft contribution from the bared collinear WF amplitude with the soft function $S\left(b_{\perp}, \mu, \delta^{+}, \delta^{\prime}\right)$. This leads to the collinear function for the incoming direction: $\psi_{\bar{q} q}\left(x, b_{\perp}, \mu, \delta^{\prime}-\right) / S\left(b_{\perp}, \mu, \delta^{+}, \delta^{\prime}-\right)$. Similarly, for the out-going direction one obtains the collinear function $\psi^{\dagger}\left(x^{\prime}, b_{\perp}, \mu, \delta^{\prime}+\right) / S\left(b_{\perp}, \mu, \delta^{\prime}+, \delta^{-}\right)$.

Here we briefly comment on the choices for the gaugelink directions in the soft functions and the WF amplitudes. Naively, the gauge-links along the $p$ direction have to be past-pointing. However, similar to the arguments in (Collins and Metz, 2004) for the SIDIS process, based on the space-time picture of collinear divergences, one can chose future pointing gauge-links along $p$ direction as well. With all the gauge-links being future pointing, the soft function equals to $S^{-}$which is manifestly real, and the WF amplitudes for the incoming and outgoing hadrons are in complex conjugation to each other.

Besides the collinear and soft functions, we still need the hard core $H_{F}\left(Q^{2}, \bar{Q}^{2}, \mu^{2}\right)$ where $Q^{2}=x x^{\prime} P \cdot P^{\prime}, \bar{Q}^{2}=$ 
$\bar{x} \bar{x}^{\prime} P \cdot P^{\prime}$ and an integral over the momentum fractions $x, x^{\prime}$ is assumed. Taking together, we have the TMD factorization of the form factor into hard, collinear and soft functions:

$$
\begin{aligned}
& F\left(b_{\perp}, P, P^{\prime}, \mu\right)=\int d x d x^{\prime} H_{F}\left(Q^{2}, \bar{Q}^{2}, \mu^{2}\right) \\
& \times\left[\frac{\psi_{\bar{q} q}^{\dagger}\left(x^{\prime}, b_{\perp}, \mu, \delta^{\prime}\right)}{S\left(b_{\perp}, \mu, \delta^{\prime}, \delta^{-}\right)}\right]\left[\frac{\psi_{\bar{q} q}\left(x, b_{\perp}, \mu, \delta^{\prime}\right)}{S\left(b_{\perp}, \mu, \delta^{+}, \delta^{\prime}-\right)}\right] \\
& \times S\left(b_{\perp}, \mu, \delta^{+}, \delta^{-}\right) .
\end{aligned}
$$

All the rapidity regulators in all the WF amplitudes and the soft functions are cancelled.

Let us consider a one-loop example. The incoming hadron state consists of a free quark with momentum $x_{0} P^{+}$and a free anti-quark with momentum $\bar{x}_{0} P^{+}$. Similarly the outgoing state consists of a pair of free quark and anti-quark with momentum $x_{0}^{\prime} P^{\prime}-, \bar{x}_{0}^{\prime} P^{\prime}-$, respectively. The spin projection operator for the incoming state is proportional to $\gamma^{5} \gamma^{-}$and for the out-going state is proportional to $\gamma^{5} \gamma^{+}$. The tree level form factor is normalized to 1. At one-loop level, the pseudo-scalar form factor with vector currents $\Gamma=\gamma^{\mu}, \Gamma^{\prime}=\gamma_{\mu}$ where a summation over $\mu$ is assumed reads:

$$
F\left(b_{\perp}, P, P^{\prime}, \mu\right)=1+\frac{\alpha_{s} C_{F}}{2 \pi} F^{(1)}\left(b_{\perp}, Q^{2}, \bar{Q}^{2}, \mu^{2}\right)
$$

where $Q^{2}=2 x_{0} x_{0}^{\prime} P^{+} P^{\prime}-, \bar{Q}^{2}=2 \bar{x}_{0} \bar{x}_{0}^{\prime} P^{+} P^{\prime}-$ and

$$
\begin{aligned}
& F^{(1)}\left(b_{\perp}, Q^{2}, \bar{Q}^{2}, \mu^{2}\right) \\
& =-7+\left(-\frac{1}{2} \ln ^{2} b_{\perp}^{2} Q^{2}+\frac{3}{2} \ln b_{\perp}^{2} Q^{2}+(Q \rightarrow \bar{Q})\right)
\end{aligned}
$$

This result can be obtained from the one-loop DY structure function (D'Alesio et al., 2014) using the substitution $\ln ^{2}\left(-Q^{2} b_{\perp}^{2}\right) \rightarrow \frac{1}{2} \ln ^{2} Q^{2} b_{\perp}^{2}+\ln ^{2} \bar{Q}^{2} b_{\perp}^{2}$ and $\ln \left(-Q^{2} b_{\perp}^{2}\right) \rightarrow \frac{1}{2} \ln Q^{2} b_{\perp}^{2}+\ln \bar{Q}^{2} b_{\perp}^{2}$. Similar to the TMD factorization for SIDIS and DY process, one should also notice that the hard kernel $H_{F}\left(Q^{2}, \bar{Q}^{2}, \mu^{2}\right)$ can be obtained from that of the space-like Sudakov form factor:

$$
H_{F}\left(Q^{2}, \bar{Q}^{2}, \mu^{2}\right)=H^{\text {sud }}\left(-Q^{2}\right) H^{\text {sud }}\left(-\bar{Q}^{2}\right)
$$

where $H^{\text {sud }}\left(-Q^{2}\right)$ is given in (Collins and Rogers, 2017). At one-loop level, we then obtain:

$$
\begin{aligned}
& H_{F}\left(Q^{2}, \bar{Q}^{2}, \mu^{2}\right)=1 \\
& +\frac{\alpha_{s}}{4 \pi}\left(-16+\frac{\pi^{2}}{3}+3 L_{Q}+3 L_{\bar{Q}}-L_{Q}^{2}-L_{\bar{Q}}^{2}\right)
\end{aligned}
$$

where $L_{Q}=\ln \frac{Q^{2}}{\mu^{2}}$ and $L_{\bar{Q}}=\frac{\bar{Q}^{2}}{\mu^{2}}$.

Now we construct the Euclidean version of the factorization in terms of the quasi-WF amplitudes, the reduced soft function, and hard contribution. The quasi-WF amplitudes are defined in a way similar to Eq. (195):

$$
\begin{aligned}
& \tilde{\psi}_{\bar{q} q}\left(x, b_{\perp}, \mu, \zeta_{z}\right)=\int \frac{d \lambda}{4 \pi} e^{i x \lambda} \\
& \frac{\left\langle 0\left|\bar{\psi}\left(\frac{\lambda n_{z}}{2}+\vec{b}_{\perp}\right) \gamma^{z} \mathcal{W}_{z}\left(\frac{\lambda n_{z}}{2}+\vec{b}_{\perp} ; L\right) \psi\left(-\frac{\lambda n_{z}}{2}\right)\right| P\right\rangle}{Z_{E}\left(2 L, b_{\perp}, \mu\right)},
\end{aligned}
$$

in which the staple-shaped gauge-link $\mathcal{W}_{z}$ is defined in Eq. (196). The gauge-links should point to $+z$ direction in accordance to the $+\infty$ choice on the light-cone side.

The factorization to the LFWF amplitude follows a similar reasoning to that of the quasi-TMDPDFs presented in previous sections. Alternatively, we can factorize it using quantities defined in on-light-cone rapidity scheme,

$$
\begin{aligned}
\tilde{\psi}_{\bar{q} q}\left(x, b_{\perp}, \mu, \zeta_{z}\right) & =H_{1}^{+}\left(\zeta_{z} / \mu^{2}, \bar{\zeta}_{z} / \mu^{2}\right) \\
& \times\left[\frac{\psi_{\bar{q} q}\left(x, b_{\perp}, \mu, \delta^{-}\right)}{S\left(b_{\perp}, \mu, \delta^{+}, \delta^{-}\right)}\right] S\left(b_{\perp}, \mu, \delta^{+}\right) .
\end{aligned}
$$

This factorization is the result of applying a similar leading-region analysis to the quasi-WF amplitude. The $\psi_{\bar{q} q}\left(x, b_{\perp}, \mu, \delta^{-}\right) / S\left(b_{\perp}, \mu, \delta^{+}, \delta^{-}\right)$re-sums all the collinear divergences, while the soft function $S\left(b_{\perp}, \mu, \delta^{+}\right)$ contains an off-light-cone direction along $n_{z}$. It re-sums the soft divergences of the quasi-WF amplitude. The soft functions $S\left(b_{\perp}, \mu, \delta^{+}, \delta^{-}\right)$and $S\left(b_{\perp}, \mu, \delta^{+}\right)$subtract away the regulator dependencies introduced in the bare LFWF amplitude. The overall combination in the righthand side of Eq. (246) is rapidity-scheme independent. Similar to the case of the form factor, we can chose all the gauge-links along the incoming collinear direction to be future-pointing.

Combining together Eqs. (240) and (246) and using the relation $\zeta \zeta^{\prime}=\zeta_{z} \zeta_{z}^{\prime}$, one obtains the form factor factorization,

$$
\begin{aligned}
& F\left(b_{\perp}, P, P^{\prime}, \mu\right) \\
& =\int d x d x^{\prime} H\left(x, x^{\prime}\right) \tilde{\psi}_{\bar{q} q}^{\dagger}\left(x^{\prime}, b_{\perp}\right) \tilde{\psi}_{\bar{q} q}\left(x, b_{\perp}\right) S_{r}\left(b_{\perp}, \mu\right)
\end{aligned}
$$

where we have only kept the $x, b_{\perp}$ dependencies of the WF amplitudes with other variables being omitted, and the hard kernel $H$ is given by:

$$
\begin{aligned}
& H\left(x, x^{\prime}\right)=H\left(\zeta_{z}, \zeta_{z}^{\prime}, \bar{\zeta}_{z}, \bar{\zeta}_{z}^{\prime}, \mu^{2}\right) \\
& \quad=\frac{H_{F}\left(Q^{2}, \bar{Q}^{2}, \mu^{2}\right)}{H_{1}^{+}\left(\zeta_{z} / \mu^{2}, \bar{\zeta}_{z} / \mu^{2}\right) H_{1}^{+}\left(\zeta_{z}^{\prime} / \mu^{2}, \bar{\zeta}_{z}^{\prime} / \mu^{2}\right)},
\end{aligned}
$$

where $Q^{2}=\sqrt{\zeta_{z} \zeta_{z}^{\prime}}$ and $\bar{Q}^{2}=\sqrt{\bar{\zeta}_{z} \bar{\zeta}_{z}^{\prime}}$. And the reduced soft function is

$$
S_{r}\left(b_{\perp}, \mu\right)=\lim _{\delta^{+}, \delta^{-} \rightarrow 0} \frac{S\left(b_{\perp}, \mu, \delta^{+}, \delta^{-}\right)}{S\left(b_{\perp}, \mu, \delta^{+}\right) S\left(b_{\perp}, \mu, \delta^{-}\right)} .
$$

It can be shown based on property of off-light-cone soft functions that $S_{r}$ defined here agrees with the one defined in Eq. (204). 
Therefore, with non-perturbative quantities $F$ and $\psi^{+}$, we obtain the reduced soft function,

$$
S_{r}\left(b_{\perp}, \mu\right)=\frac{F\left(b_{\perp}, P, P^{\prime}, \mu\right)}{\int d x d x^{\prime} H\left(x, x^{\prime}\right) \tilde{\psi}_{\bar{q} q}^{\dagger}\left(x^{\prime}, b_{\perp}\right) \tilde{\psi}_{\bar{q} q}\left(x, b_{\perp}\right)},
$$

where $H$ can be obtained perturbatively.

Based on the one-loop results for the form factor, the quasi-WF amplitudes and the reduced soft function, the one-loop matching kernel for the vector current can be extracted as:

$$
\begin{aligned}
& H\left(\zeta_{z}, \zeta_{z}^{\prime}, \bar{\zeta}_{z}, \bar{\zeta}_{z}^{\prime}, \mu^{2}\right)=1+\frac{\alpha_{s} C_{F}}{2} i \ln \frac{\sqrt{\zeta_{z} \bar{\zeta}_{z}}}{\sqrt{\zeta_{z}^{\prime} \zeta_{z}^{\prime}}} \\
& +\frac{\alpha_{s} C_{F}}{4 \pi}\left(-8+\ln ^{2} \frac{\sqrt{\zeta_{z}}}{\sqrt{\zeta_{z}^{\prime}}}+\ln \frac{\sqrt{\zeta_{z} \zeta_{z}^{\prime}}}{\mu^{2}}+(\zeta \rightarrow \bar{\zeta})\right),
\end{aligned}
$$

and the renormalization group equation for $H$ reads:

$$
\mu^{2} \frac{d}{d \mu^{2}} \ln H\left(\zeta_{z}, \zeta_{z}^{\prime}, \bar{\zeta}_{z}, \bar{\zeta}_{z}^{\prime}, \mu^{2}\right)=-2 \gamma_{F}\left(\alpha_{s}\right)-\Gamma_{S}\left(\alpha_{s}\right),
$$

where $\gamma_{F}$ and $\Gamma_{S}$ have been defined before.

Here we briefly comment on the end-point behavior. As $x \sim 0$, the hard kernel diverges logarithmically near the end point as $1+\alpha_{s} \ln ^{2} x$, but the quasi-WF amplitudes approach zero at large or small $x$ linearly, thus the end point regions behave as $x \ln ^{2} x$, which is free from those problems for the $k_{T}$ factorization for electromagnetic form factor ( $\mathrm{Li}$ and Sterman, 1992). Moreover, we can fix the $z$-component momentum transfer at each of the vertices to be $P^{z}$, which indicating that $x+x^{\prime}=1$. In this case the end-point behavior is improved to $x^{2} \ln ^{2} x$.

\section{LATTICE PARTON PHYSICS WITH LAMET}

Lattice gauge theory simulates continuum QCD in imaginary time on a discretized 4D Euclidean lattice. The method is characterized by the finite lattice spacing $a$ and volume $L_{1} \times L_{2} \times L_{3} \times T$, and input parameters such as the strong coupling and quark masses. To calculate physical quantities, one usually expects to take the continuum $(a \rightarrow 0)$ and infinite volume $L_{i}, T \rightarrow \infty$ limits, as well as tuning the quark masses so that observables such as the pion mass $m_{\pi}$ agrees with the physical value of $\sim 140 \mathrm{MeV}$. There are different methods to implement the fermions on the lattice (Rothe, 1992), which leads to different properties of the lattice action such as chiral symmetry breaking for Wilson fermions. In the lattice calculation of hadron matrix elements, the initial and final states are generated by acting the source and sink interpolation operators on the vacuum, and the groundstate contributions are filtered out by propagating over a sufficiently large Euclidean time. A boosted hadron state can be obtained by inserting momentum into the source and sink operators through Fourier transform in the 3D spatial coordinates.

The lattice QCD calculations of parton physics using LaMET started with the exploratory studies on the simplest PDFs and the gluon helicity (Alexandrou et al. , 2015; Lin et al., 2015; Yang et al., 2017), which yielded fairly encouraging results, demonstrating that LaMET is a viable approach. In subsequent studies, more attention has been paid to the systematics, including establishing a proper renormalization and matching procedure, simulating at the physical pion mass, removing the excited-state contamination, etc. Such studies have greatly improved the precision of the calculations, with the latest results exhibiting a reasonable agreement with phenomenological PDFs (Alexandrou et al., 2018a,b; Gao et al., 2020; Izubuchi et al., 2019; Lin et al., 2018a). In the meantime, explorations have also been made on similar large momentum data using the coordinate-space factorization methods including the pseudo-PDF (Joó et al., 2019a, b), 2020; Orginos et al., 2017) and current-current correlation (Bali et al., 2019; Sufian et al., 2020, 2019). Nevertheless, dedicated large-scale efforts with the state-of-art resources are yet to be seen. Lattice parton physics with LaMET is just at its dawn. With EIC in the US going forward, a new era of lattice calculations is to come.

In this section, we summarize the current status of lattice calculations using LaMET and discuss future prospects. We will begin with a general discussion on what kind of lattice setups are best suited for LaMET calculations, and then briefly summarize relevant lattice techniques that facilitate such calculations. After that, we review the lattice calculations that have been carried out so far and point out future improvements. A nice complementary discussion about lattice calculations has been made in (Cichy and Constantinou, 2019). Other reviews that summarize the recent developments in the lattice calculation of PDFs can be found in Constantinou, 2020; Monahan, 2018a; Zhao, 2020).

\section{A. Special Considerations for Lattice Calculations}

In this subsection, we discuss the challenges for lattice calculations in LaMET, and estimate the required lattice requirements by taking the collinear PDFs as an example.

\section{Challenges due to large momentum}

In addition to common challenges with other lattice calculations, such as taking the continuum and infinite volume limits, simulating at or extrapolating to the physical pion mass, etc., LaMET applications require generating large-momentum hadron on lattice. For LaMET expansion, $1 /\left(x P^{z}\right)$ is the expansion parameter, and 


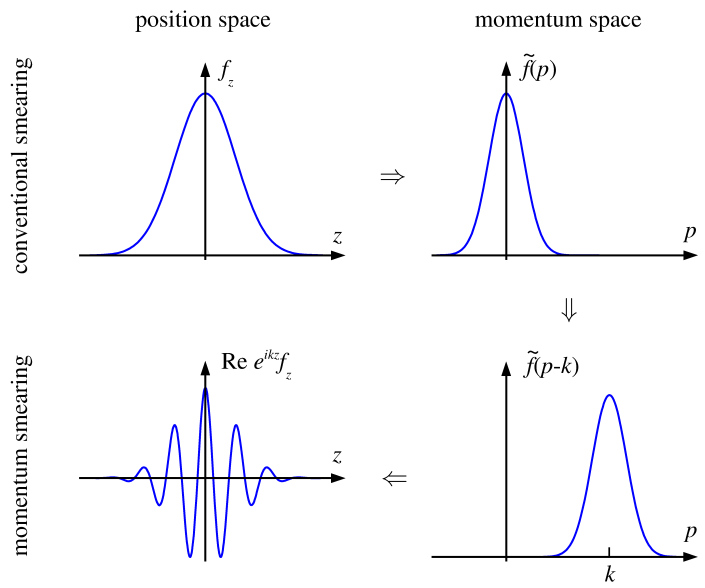

FIG. 15: Conventional smearing (left) versus momentum smearing (right) (Bali et al., 2016): Conventional smearing has small overlap with high momentum state. Momentum smearing shifts momentum to peak at nonzero value in momentum space.

for the coordinate-space factorizations, large quasi-lightcone distance $\lambda$ requires even bigger hadron momentum. However, realizing this faces a number of practical challenges. First, it has been difficult to generate largemomentum hadron states on the lattice, until the technique of momentum smearing (Bali et al., 2016) was proposed. The conventional smearing method in coordinate space is designed to increase the overlap with ground state hadron at rest. Thus, it is not surprising that such a smearing is not efficient when the hadron has a large momentum. The momentum smearing technique introduces an extra phase factor $e^{i \vec{k} \cdot \vec{z}}$ to the quark field, such that it is peaked at nonzero momentum $\vec{k}$ in Fourier space, as shown in Fig. 15. In this way, the overlap with high momentum state is vastly increased after Euclidean time evolution. Recently, the momentum smearing technique has been incorporated into the framework of distillation (Egerer et al., 2020) to improve the extraction of ground-state energy and matrix elements at momentum $\lesssim 3 \mathrm{GeV}$. Although there are other proposed methods to generate large momentum (Wu et al., 2018), the momentum smearing has become a standard technique in LaMET applications.

Second, the proton size is frame-dependent and changes with its momentum. In the proton's rest frame, simulating its structure requires that the lattice spacing be much smaller than the QCD confinement scale, i.e. $a \ll \Lambda_{\mathrm{QCD}}^{-1}$. When the proton is moving fast, it undergoes Lorentz contraction by a boost factor $\gamma$ in the momentum direction, thus a finer lattice spacing $a \ll\left(\gamma \Lambda_{\mathrm{QCD}}\right)^{-1}$ is needed. If $a \leq 0.2 \mathrm{fm}$ is the minimum requirement to investigate a static proton, one will need at least $a \leq 0.04 \mathrm{fm}$ to have the same resolution for a proton at $5 \mathrm{GeV}$. A smaller lattice spacing is difficult to achieve with current computing resources, for it suf- fers from the well-known critical slowing down problem, i.e., the auto-correlation times of observables such as the topological charge increase when approaching the continuum limit (Schaefer et al., 2011), which can be much longer than the Monte-Carlo simulation times. A lattice with open (Neumann) boundary condition on gauge fields in the Euclidean time direction (Luscher and Schaefer, 2011), which allows topological charge to flow in and out at boundaries of time, may overcome this problem.

Third, the gaps between the ground state and the excited state energies become smaller because of the time dilation effect. In the proton's rest frame, the excited state contamination exponentially decays with the mass gap $\Delta M$ and evolution time $\tau$ in the form of $e^{-\Delta M \tau}$. In the boosted frame, the mass gap $\Delta M$ in the decay factor is replaced by the energy gap $\Delta E \sim \Delta M / \gamma$, and the decay changes like $e^{-\Delta M \tau} \rightarrow e^{-\Delta E \tau}=e^{-\Delta M \tau / \gamma}$ under Euclidean time evolution. Therefore, with a boosted state, a longer time evolution (source-sink separation) is needed. For example, if a source-sink separation of $1 \mathrm{fm}$ is needed to separate the excited state of proton with 2 $\mathrm{GeV}$ momentum, a proton with $5 \mathrm{GeV}$ momentum will require a source-sink separation of $2.5 \mathrm{fm}$. Even if the two-state fit technique is used, a longer time evolution is still required so that only the ground and first excited states dominate.

Last but not the least, lattice calculation requires $P^{z} \ll 1 / a$, so that discretization effects of $\mathcal{O}\left(\left(a P^{z}\right)^{n}\right)$ are under control. Therefore, one has to go to smaller lattice spacing in order to reach larger momentum. The quantification of $\mathcal{O}\left(\left(a P^{z}\right)^{n}\right)$ effects alone in LaMET calculations has not been done, as all discretization errors are treated on equal footing in continuum extrapolation.

To summarize, to achieve a precision calculation of boosted hadron structure on lattice, a fine lattice spacing (at least in the longitudinal direction) and a large box size in the time direction are essential, which of course will also require control over the signal-to-noise ratio at large Euclidean times.

\section{Considerations for lattice setup}

In practical calculations, a correlation function is first obtained on lattice in coordinate space, and then Fourier transformed to momentum space with the phase factor $e^{i \lambda x}$ where $\lambda=z P^{z}$. Therefore, the smallest $x$ one can reach can be roughly estimated from the largest $\lambda$ as $x \sim 1 / \lambda$. However, a more stringent constraint comes from requiring that the higher-twist contribution $\mathcal{O}\left(\Lambda_{\mathrm{QCD}}^{2} /\left(x P^{z}\right)^{2}\right)$ be small so that the factorization is still valid, which implies $x \gg \Lambda_{\mathrm{QCD}} / P^{z}$. This also provides a rough estimate for the largest attainable $x$ $\left(x \ll 1-\Lambda_{\mathrm{QCD}} / P^{z}\right)$ since the momentum fraction carried by other partons is $\sim(1-x)$ which should also be bounded from below by the above estimate. 
For the current state-of-the-art simulations, the lattice spacing can reach $0.04 \mathrm{fm}$ (Fan et al., 2020a; Gao et al., 2020), which implies $P_{\max }^{z} \sim 5 \mathrm{GeV}$ and the effective resolution in longitudinal direction is about $\gamma a \sim 0.2 \mathrm{fm}$. Thus the valid $x$ region that can be extracted from lattice is roughly 0.1 to 0.9 . On the other hand, to avoid finite volume effects, it is believed that $m_{\pi} L \gtrsim 4$. For physical pion mass, the box size in spatial direction $L$ should be at least $6 \mathrm{fm}$, which means the box size is 150 lattice spacing. So far, the largest box size in LaMET calculations is 5.8 $\mathrm{fm}$ (Lin et al., 2018a). As discussed in Sec. VI.A.1, the source-sink separation of $2.5 \mathrm{fm}$ is needed for $P^{z}=5$ $\mathrm{GeV}$. So the box size in time direction $T$ does not need to be particularly longer than $L$, and $T=L$ is sufficient in this lattice setup. In summary, with $a=0.04 \mathrm{fm}$ at physical pion mass, one need a $L^{3} \times T=150^{3} \times 150$ lattice to reliably extract $0.1<x<0.9$ region, which could be possible in an exa-scale computer.

There are potential tricks to reduce the computational cost. First, the required source-sink separation can be shorter if one uses a multi-state instead of two-state fit with enough statistics. However, since the number of fitting parameters in $n$-state fit grows as $n^{2}$, such a fitting will become infeasible for too large $n$. Second, note that the resolution required for transverse proton structures is not affected by the Lorentz boost, one may use a coarse lattice in the transverse directions, $a_{\perp}=0.1 \mathrm{fm}$. The required box size is then $L_{\|} \times L_{\perp}^{2} \times T=150 \times 60^{2} \times 150$. This asymmetric lattice can greatly reduce the resources needed for large momentum since the transverse box size is fixed. However, generating configurations and renormalization on such a lattice might bring new problems and should be further studied.

In the near future, exascale supercomputers may help to reach higher momentum, as large as $5 \mathrm{GeV}$ for the proton, and improve the precision of LaMET calculations. Further theoretical developments and new ideas on the technique and algorithms are also needed to overcome the simulation difficulties.

\section{B. Non-Singlet PDFs}

In this subsection, we review current status of lattice calculations of flavor non-singlet (isovector) PDFs in the proton and pion. The non-singlet case has the advantage that the mixing with gluons as well as the lattice calculation of disconnected diagrams can be avoided, thus greatly reducing the computational challenge. It is the most extensively studied parton observable with LaMET so far.

\section{Proton}

The pioneering lattice studies for the isovector quark PDF in the proton were carried out in Alexandrou et al., 2015; Lin et al., 2015). These are proof-of-principle studies as the renormalization of quasi-PDFs was not well understood at that time. Nevertheless, their results encouraged the follow-up theoretical works on LaMET, including a proper renormalization and matching suitable for lattice implementations.

Certain lattice artifacts have also been studied. For example, although there is no power-divergent mixing for the quasi-PDF operators on the lattice, additional operator mixings that are not seen in the continuum can still occur if a non-chiral lattice fermion such as the Wilson-type fermion is used. In (Chen et al. 2019a; Constantinou and Panagopoulos, 2017) it was shown that at $\mathcal{O}\left(a^{0}\right)$ the operator for the unpolarized quark quasi-PDF, $O_{\gamma^{z}}(z)$, can mix with the scalar operator $O_{1}(z)$, whereas $O_{\gamma^{t}}(z)$ does not. To reduce the systematic uncertainty from such mixing, $\Gamma=\gamma^{t}$ has been used since then for lattice calculations of the unpolarized quark PDF, e.g. in Alexandrou et al., 2017b; Chen et al., 2018; (Green et al., 2018). Similarly, for helicity and transversity cases, one should choose $\Gamma=\gamma^{5} \gamma^{z}$ and $\Gamma=i \sigma^{z \perp}=\gamma^{\perp} \gamma^{z}$, respectively, in order to avoid the mixing. It should be noted that at $\mathcal{O}(a)$ all $\tilde{O}_{\Gamma}(z)$ 's can mix with others (Chen et al., 2019a). Nevertheless, a fine lattice spacing can reduce these effects.

In Alexandrou et al., 2017b; Chen et al., 2018; Green et al., 2018), the nonperturbative renormalization (NPR) of the quasi-PDFs was studied in the RI/MOM scheme (Martinelli et al., 1995). This scheme has several advantages: The lattice regularization scheme can be converted to $\overline{\mathrm{MS}}$ scheme through $\mathrm{RI} / \mathrm{MOM}$ renormalization condition, the computation cost is affordable, the systematic errors can be reduced or quantified more easily, etc. The works before 2018 did not include NPR and the systematics were not accurately quantified. The later works have implemented the RI/MOM scheme and the corresponding perturbative matching Constantinou and Panagopoulos, 2017; Liu et al., 2020; Stewart and Zhao, 2018). The coordinate-space method is also developed in parallel in (Bhat et al., 2020; Cichv et al., 2019; ;Joó et al., 2019a, 2020; Orginos et al., 2017). In Figs. 16 and 17, we select some most recent lattice results. ETMC published the proton unpolarized, helicity and transversity PDFs with $P^{z}=$ $1.4 \mathrm{GeV}$ at physical pion mass (Alexandrou et al., 2018a, $\mathrm{b}$ ), and $\mathrm{LP}^{3}$ published the proton helicity PDF with unprecedented momentum $P^{z}=3.0 \mathrm{GeV}$ at physical pion mass (Lin et al., 2018a). Recently, calculations on fine lattices (Alexandrou et al., 2020c; Fan et al. ,2020a) and an extrapolation to the continuum limit (Alexandrou et al., 2020c) have become available. The finite volume effects, which was first studied in a 
model (Briceño et al., 2018), have also been investigated on the lattice lately in (Lin and Zhang, 2019), where no sizeable volume-dependence was observed at $P^{z}=1.3$ and $2.6 \mathrm{GeV}$.

The PDFs extracted from LaMET can be useful for phenomenology by providing input in kinematic regions that are difficult to measure in experiments. It has attracted attention from global fit community (Bringewatt et al., 2020; Constantinou et al., 2020; Hobbs et al., 2019; Lin et al., 2018c). For example, it has been found that in the large- $x$ region of unpolarized PDF the lattice result will lead to significant improvement on global fit result if it reaches an accuracy of about 10\% (Lin et al., 2018c). The sea quark asymmetry (Geesaman and Reimer, 2019) is also possible to be investigated now directly on lattice. For the transversity PDF, due to the difficulty of measurement in experiment, lattice results can already have impact on improving global fit and even making predictions. In addition to the isovector cases, calculations of the strange and charm unpolarized distributions (Zhang et al., 2020c), as well as the flavor separation of light quarks in the helicity PDF (Alexandrou et al., 2020a), have also been carried out recently. From early exploratory results showing qualitative behavior of PDFs to the latest results which are comparable with global fits, it has come a long way in developing new techniques (momentum smearing, renormalization, matching, etc.) and the computation resources have been steadily increased over time. The systematic uncertainties in the lattice calculation of PDFs have been thorougly investigated by the ETMC (Alexandrou et al., 2019b). Further studies on systematics such as the discretization effects and finite volume effects on various lattice ensembles are still necessary. In the future, lattice QCD is expected to make a significant impact on nuclon structure.

To conclude this subsection, we would like to mention that there are also lattice studies of the isovector PDF of other baryons, $\Delta^{+}$to be more concrete, using LaMET (Chai et al., 2020).

\section{Pion}

The pion valence quark distribution has been extracted from various Drell-Yan data for pion-nucleon/pionnucleus scattering, while theoretical predictions do not yield consistent results with the experimental extraction, especially in large- $x$ region (Holt and Roberts, 2010). LaMET calculations will be able to shed valuable light on how to resolve this disagreement, provided that all systematics are well under control.

In principle, calculating the pion valence PDF is easier than the proton PDF. First, the pion state is easier to produce and the quark contractions are fewer. Second, the energy gap between the first excited and ground
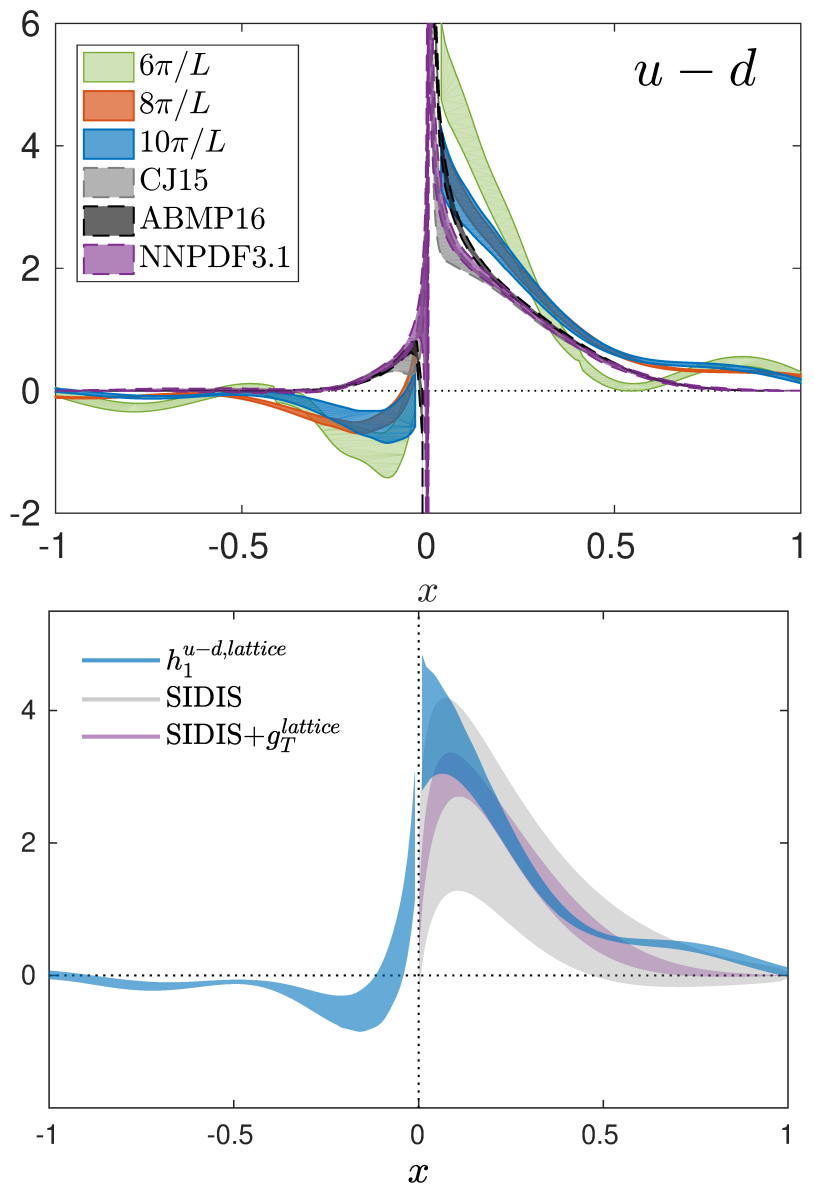

FIG. 16: Proton isovector quark PDF Alexandrou et al., 2018a b): The unpolarized PDF with $P^{z}$ from 0.82 to $1.4 \mathrm{GeV}$ and the transversity PDF with $P^{z}=1.4 \mathrm{GeV}$ are in upper and lower figures. CJ15 (Accardi et al., 2016a),

ABMP16 (Alekhin et al., 2017), and NNPDF3.1 (Ball et al., 2017) are global fits. SIDIS is global fit and SIDIS $+g_{T}^{\text {lattice }}$ is global fit with lattice constraint on tensor charge $g_{T}^{\text {lattice }}$ (Lin et al., 2018b).

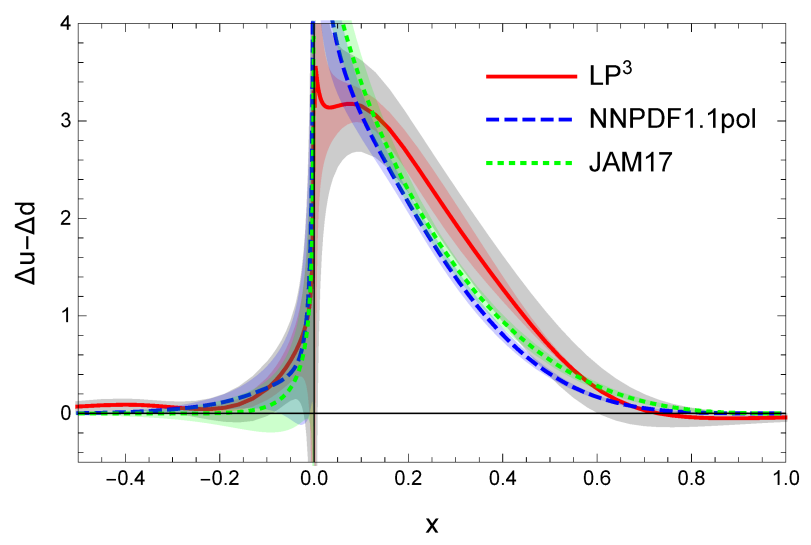

FIG. 17: The proton isovector quark helicity PDF $\left(P^{z}=3.0\right.$ $\mathrm{GeV}$ ) (Lin et al., 2018a) with red band for statistic error and grey band for statistic and systematic errors.

NNPDF1.1pol (Nocera et al., 2014) and JAM17 (Ethier et al., 2017) are global fits. 


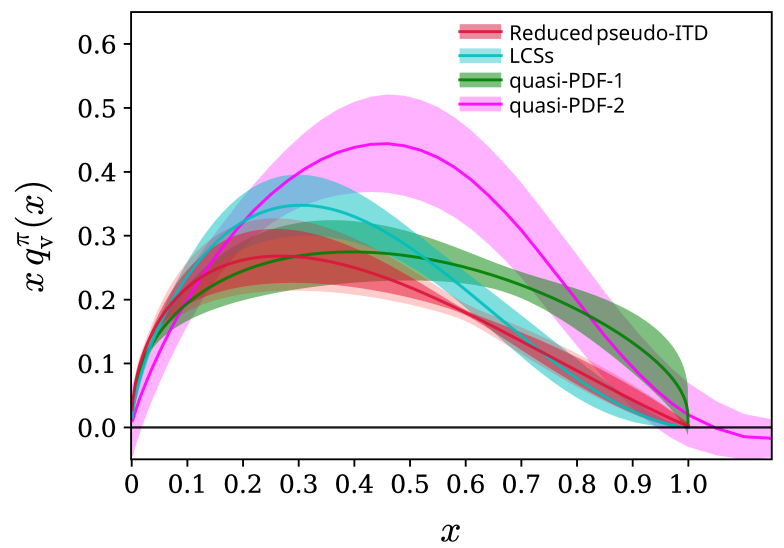

FIG. 18: Pion valence quark PDFs in various approach: Compare the results of pesudo-PDF [Reduced pseudo-ITD (Joó et al., 2019b)], quasi-PDF [quasi-PDF-1 (Izubuchi et al., 2019) and quasi-PDF-2 (Zhang et al., 2019a)], and the current-current correlator approach [LCSs (Sufian et al., 2019)].

state of the pion is much bigger than the energy gap of the proton. Therefore, the excited-state contamination is easier to control. The simulation was first performed in (Zhang et al., 2019a) with the same lattice setup and procedure used in exploratory studies of the proton PDF. A more thorough study on the pion valence quark PDF was done by the lattice QCD group of BNL (Izubuchi et al., 2019). It is worth pointing out that the excited state contamination was thoroughly studied using multi-state fits, with the ground and first excited states both agreeing with the expected dispersion relations, indicating that the excited contamination is well under control. The comparison of the lattice results from quasi-PDF, pseudo-PDF and current-current correlator approach are shown in Fig. 18, Note that the $\mathrm{LP}^{3}$ (Zhang et al., 2019a) result was obtained using Fourier transformation and inversion of factorization formula, while other three groups used parameterization models to fit the lattice data. More dedicated effort is needed to reduce the errors, and a meaningful comparison between different operators and analysis methods should be made.

For other mesons, we would like to mention that there is a study of kaon valence quark PDF using MILC configurations (Lin et al., 2020).

\section{Gluon Helicity and Other Collinear Parton Properties}

In this subsection, we summarize the applications of LaMET to other collinear parton observables, including the gluon helicity, the gluon PDFs, meson DAs and GPDs.

\section{Total gluon helicity}

The total gluon helicity $\Delta G$ is a key component in understanding the proton spin structure. It has been intensively explored at RHIC and will be dedicatedly pursued at EIC in the future. However, a theoretical lattice calculation of $\Delta G$ had not been possible until the proposal of LaMET.

The first such effort was made by $\chi \mathrm{QCD}$ collaboration in (Yang et al., 2017). The calculation was carried out with valence overlap fermions on $2+1$ flavor domain-wall fermion gauge configurations, using ensembles with multiple lattice spacings and volumes including one with physical pion mass. The authors simulated proton matrix elements of the free-field operator $(\vec{E} \times \vec{A})^{3}$ in the Coulomb gauge at various momenta, and then converted them to the $\overline{\mathrm{MS}}$ scheme with one-loop lattice perturbation theory. The $\overline{\mathrm{MS}}$ matrix elements at each lattice momentum are shown in Fig. 19, Though a LaMET matching is necessary to match the results to the physical gluon helicity, the authors did not apply it due to the concern of perturbative convergence of the matching coefficient (Ji et al., 2015c). Instead, as the $\overline{\mathrm{MS}}$ matrix elements show rather mild momentum dependence up to the maximum momentum $\sim 1.5 \mathrm{GeV}$, they extrapolated the results to infinite momentum, as well as physical pion mass and continuum limits, with a model motivated by chiral EFT. Their final result is $\Delta G\left(\mu^{2}=10 \mathrm{GeV}^{2}\right)=0.251(47)(16)$, or $50(9)(3) \%$ of the total proton spin, which agrees with the truncated moment of $\Delta g(x)$ (de Florian et al., 2014; Nocera et al., 2014) within uncertainties.

Despite such progress, one should be cautious that this calculation still needs further improvements in the future. Among others, the most important ones are simulations at larger proton momentum, performing an NPR and investigating perturbative convergence of LaMET matching and its implementation.

\section{Gluon PDF}

The gluon PDF is of great interest not only for precision physics at LHC, but also for understanding the gluonic structure of the proton and nucleias well as the small- $x$ dynamics - at the future EIC. With the recent progress on the renormalization and matching for gluon quasi-PDFs (Li et al., 2019; Wang et al., 2019b; Wang and Zhao, 2018; Wang et al., 2018; Zhang et al., 2019b) or the coordinate-space "pseudo distributions" (Balitsky et al., 2019), a systematic lattice calculation of the gluon PDFs can be carried out in principle.

Before the above theoretical developments, an exploratory lattice study of the proton and pion unpolarized gluon PDFs were carried out in (Fan et al., 2018). 


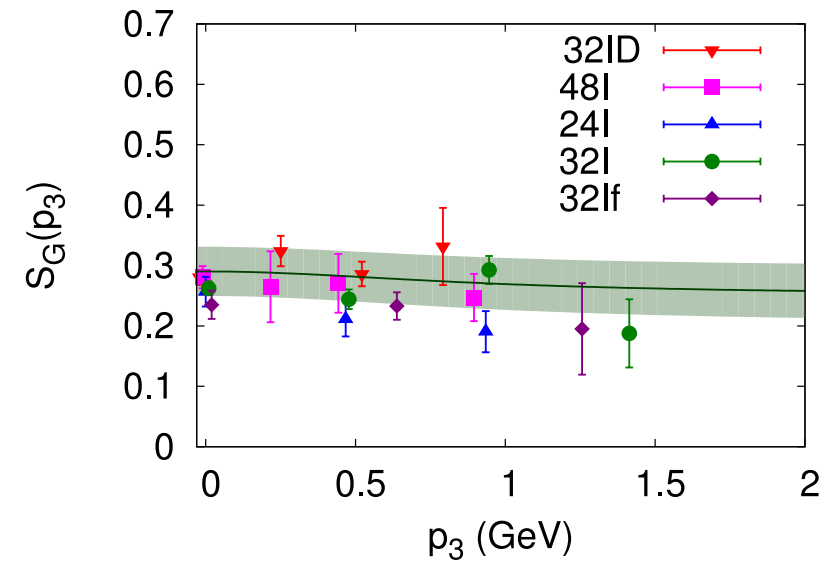

FIG. 19: Total gluon helicity (Yang et al., 2017): The results are extrapolated to the physical pion mass and continuum as a function of the proton momentum $p_{3}$ on all the five ensembles indicated by different colors of the data points.

The authors calculated quasi gluon LF correlations and compared them to the LF correlations for the gluon PDFs. Later on, based on the multiplicative renormalizability of certain choice of the quasi gluon LF correlator (Zhang et al., 2019b), the same authors used the ratio scheme (Balitsky et al., 2019) in coordinate space to renormalize the lattice matrix elements, and fitted the proton unpolarized gluon PDF with a simple two-parameter model (Fan et al., 2020b). Although the results show agreement with the global analyses in the large- $x$ region, the systematics from the modeldependence of the fit remains to be quantified for a controlled calculation of the gluon PDF.

\section{DA}

According to Sec. IV.B LaMET can be readily applied to calculating DAs, and the lattice resource needed is expected to be cheaper than that for PDFs since there is one less external state, which reduces the number of contractions for the quark propagators. So far there are a few exploratory investigations on meson DAs, in particular, on pion (Zhang et al., 2017) and kaon DAs (Zhang et al., 2019c). The lattice calculations of pion (Zhang et al., 2017) and kaon DAs (Zhang et al., 2019c) were first explored without the NPR and the corresponding matching. Recently, the pion and kaon DAs from the RI/MOM scheme analysis are extrapolated to the continuum limit (Zhang et al., 2020b), where the authors eventually adopted a two-parameter model to fit the final result. The above results are shown in Fig. 20. Apart from LaMET, the currentcurrent correlation methods (Braun and Müller, 2008; Braun et al., 2015; Detmold and Lin, 2006) have also made much progress on the pion DA (Bali et al., 2019; Detmold et al., 2020, 2018).

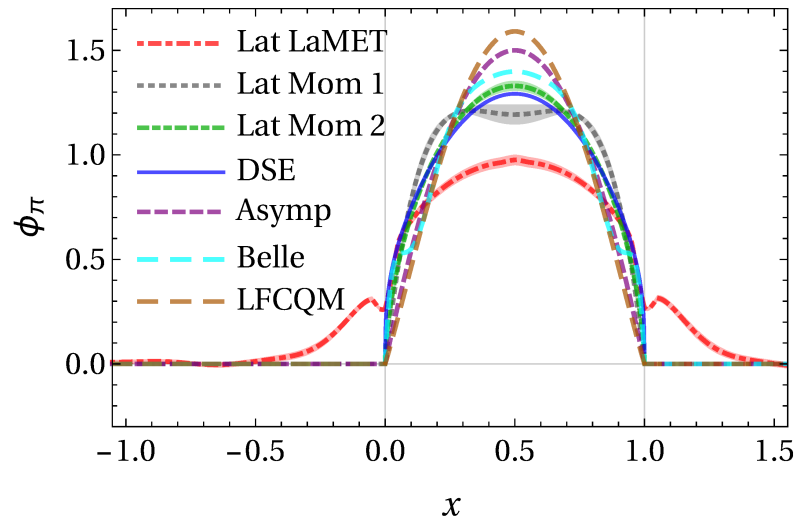

FIG. 20: Pion DA (Zhang et al., 2019c): Comparison of $\phi_{\pi}$ (Lat LaMET) to previous determinations in the literature. Lat Mom 1 and 2 are parameterized fits to the lattice moments (Braun et al., 2015); DSE is Dyson-Schwinger equation

calculations (Chang et al., 2013); Asymp is the asymptotic form $6 x(1-x)$; Belle is a fit to the Belle data (Agaev et al., 2012); LFCQM is light-front constituent quark model (de Melo et al., 2016).

\section{GPD}

As discussed in Sec. IV the global fitting of GPDs still faces challenges from their complicated kinematic dependence and limited information from the experimental observables despite the progress made (Favart et al., 2016; Kumericki et al., 2016). On the other hand, previous lattice QCD method is only able to calculate the lowest few moments of the GPDs (Hagler, 2010), which is far from sufficient to reconstruct their full kinematic dependence. Applying LaMET to GPD calculations will provide important information on the GPDs, especially in kinematic regions that are not accessible in currently available experiments. In addition, on the lattice one can study the GPD dependence on one kinematic variable by fixing the others. All these will help differentiate commonly used models in GPD parameterization.

Calculating the quasi-GPDs requires more resources than quasi-PDF, but does not need further techniques in principle. Besides, the lattice renormalization factors for the quasi-PDFs can be used here, as has been argued in Sec. IV. The first lattice calculation of the pion unpolarized isovector quark GPD was carried out in (Chen et al., 2019b), though the results are not yet able to differentiate models or compare to experiments. Recently, ETMC completed the first proof-ofprinciple calculation of the proton unpolarized and helicity GPDs (Alexandrou et al., 2020d), as shown in Fig. 21, which demonstrates that it is feasible to extract the GPDs with controlled systematics on available computational resources. 


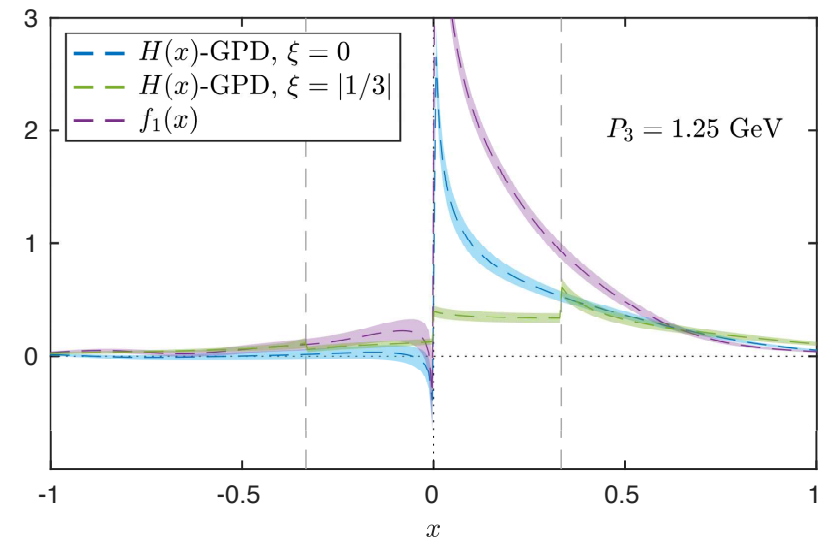

FIG. 21: Proton unpolarized isovector quark GPD (Alexandrou et al., 2020d) $H(x, \xi, t)$ for $t=-0.69 \mathrm{GeV}^{2}$ extracted from quasi-GPDs at $P_{3}=1.25 \mathrm{GeV}$, which is compared to the unpolarized PDF $f_{1}(x)$.

\section{Higher-twist PDF}

The higher-twist PDFs probe multi-parton correlations, and their contribution at $x=0$ can shed light on the LF zero modes (Ji, 2020). As we discussed in Sec. IV] such distributions can also be calculated on the lattice with the LaMET approach.

The first attempt to calculate the isovector twist-three PDF $g_{T}(x)$ has been carried out by ETMC (Bhattacharva et al., 2020a) using the one-loop matching coefficient they computed in (Bhattacharya et al., 2020b.c). Their results show agreement with the Wandzura-Wilczeck approximation (Wandzura and Wilczek, 1977), which ignores the contribution from dynamical twist-three contributions, and the Burkhardt-Cottingham sum rule Burkhardt and Cottingham, 1970). Nevertheless, the mixing between $g_{T}(x)$ and other twist-three distributions was not considered, and further study is still required for an accurate matching to the light-cone PDF.

\section{TMDs}

With tremendous experimental focus on the TMDPDFs for studying 3D proton structures and gluon saturation at EIC, their first-principle calculation from lattice QCD will significantly boost this direction by providing useful nonperturbative inputs for all the phenomenological analyses.

In this subsection, we discuss the status and prospects of calculating the quasi-TMDPDF and soft function with LaMET. Besides, we note that before LaMET there had already been efforts to extract information of TMDs by studying ratios of the lattice correlators (Engelhardt et al., 2016; Hagler et al., 2009;
Musch et al., 2012, 2011; Yoon et al., 2017), which has made a series of progress in the past decade. We begin with a brief review of them.

\section{Pre-LaMET study — ratio of lattice correlators}

By employing Lorentz covariance, the $x$-moments of TMDPDFs are related to the form factors of spacelike staple-shaped gauge link operators, which can be directly simulated on the lattice. Although the lattice calculation of the soft function was not available during that time, ratios of the spin-dependent and the unpolarized matrix elements were formed to cancel it, thus providing useful information of different TMDPDFs. For example, the time-reversal odd TMDPDFs can be studied with the staple-shaped gauge link operator in a transversely polarized proton state, thus helping understand properties related to singlespin asymmetry (SSA), which was measured experimentally at STAR (Adamczyk et al., 2016) and COMPASS (Aghasvan et al., 2017). In (Engelhardt et al., 2016; Musch et al., 2012), the Sivers and Boer-Mulders functions of proton and pion were studied; Other timereversal even functions, such as the worm-gear function $g_{1 T}$ (Tangerman and Mulders, 1995), were also studied (Yoon et al., 2017).

\section{Quasi-TMDPDF and Collins-Soper kernel}

The lattice calculation of the quasi-TMDPDF defined in Eq. (195) is straightforward. The matrix element of the staple-shaped quark Wilson line operator can be simulated the same way as the quasi-PDF case, except that the geometry of the gauge-link is different, while the calculation of Wilson loop $Z_{E}$ is standard practice in lattice QCD. The more challenging part, however, is the renormalization of the quasi-TMDPDF and its matching to the $\overline{\mathrm{MS}}$ scheme.

Using the auxiliary field theory formalism, one can argue that staple-shaped quark Wilson line operator is also multiplicatively renormalizable (Ebert et al., 2020b; Green et al., 2020). On a non-chiral lattice, it suffers from finite mixing with other quark bilinear operators, as was predicted by one-loop lattice perturbation theory (Constantinou et al., 2019). The full mixing pattern for such operators with different Dirac matrices have been studied in the RI/MOM scheme on three quenched lattice ensembles with different spacings (Shanahan et al., 2019), and a diagonalization of the mixing matrix is adopted to renormalize these operators. Meanwhile, the one-loop conversion factors that convert the RI/MOM matrix elements to the $\overline{\mathrm{MS}}$ scheme have been calculated in continuum perturbation theory for both the $z=0$ (Constantinou et al., 2019) and $z \neq 0$ (Ebert et al. 
2020b) cases.

Although the soft function is still needed to fully determine the physical TMDPDF, the $\overline{\mathrm{MS}}$ quasi-TMDPDF can already be used to extract the Collins-Soper kernel according to Eq. (210) (Ebert et al., 2019a, b; Ji et al., 2015b). Since the Collins-Soper kernel can be defined from both the bare TMDPDF and the soft function, it is independent of the external state and can be calculated in a pion which is the least expensive on the lattice. Up to mass corrections suppressed by the momentum in Eq. (209), this calculation also allows for using an unphysical valence pion mass, as long as the sea quark masses are physical.

With the method developed in (Ebert et al., 2019a), the first exploratory lattice calculation of the CollinsSoper kernel was performed in (Shanahan et al., 2020) on a quenched lattice with heavy valence pion mass $m_{\pi} \sim 1.2 \mathrm{GeV}$, and the result is shown in Fig. 22 As one can see, the lattice prediction is robust for $0.1 \mathrm{fm}<b_{\perp}<$ $0.8 \mathrm{fm}$, which covers the nonperturbative region that is important for TMD evolution in global analyses. Besides, at small $b_{\perp}$, the perturbative calculation can serve as a calibration for estimating the systematic uncertainties, as there are power corrections of $\mathcal{O}\left(1 /\left(P^{z} b_{\perp}\right)\right)$ which can only be reduced with larger $P^{z}$. In (Zhang et al., 2020a), the Collins-Soper kernel has also been extracted from a pion quasi-TMD DA, where the lattice renormalization was left out, and the result is in agreement with (Shanahan et al., 2020) within errors for a wide range of $b_{\perp}$. With improved lattice ensembles and systematic corrections in the future, it is promising to have a precise determination of the Collins-Soper kernel for TMD phenomenology.

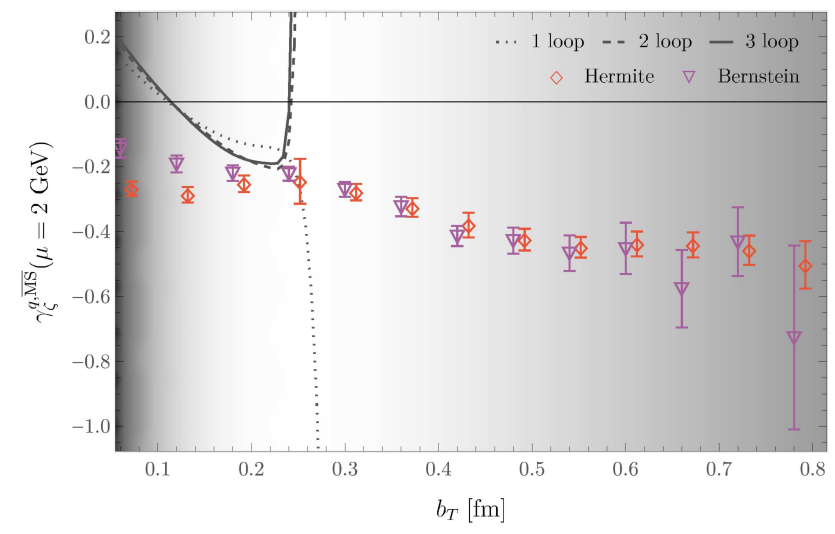

FIG. 22: The Collins-Soper kernel from the first exploratory calculation on a quenched lattice (Shanahan et al., 2020). The results are obtained by using fits to the $\overline{\mathrm{MS}}$ unsubtracted quasi-TMDPDFs with Hermite and Bernstein polynomial bases. The solid and dashed lines are the perturbative predictions ( $\mathrm{Li}$ and $\mathrm{Zhu}$, 2017; Vladimirov, 2018), which is hit the Landau pole near $b_{\perp} \sim 0.25 \mathrm{fm}$. The background shading density is proportional to a naive estimate of the power corrections $1 /\left(b_{\perp} P^{z}\right)+b_{\perp} / L$.

\section{Soft function}

As the remaining piece towards physical TMDPDFs, the soft function must be calculated in lattice QCD. In particular, the reduced soft function in Eq. (204) eliminates the regulator-scheme-dependence of the off-thelight-cone quasi-TMDPDF, so its calculation alone has great physical significance. According to Secs. (V.C) and (V.D), two methods have been proposed to calculate the off-the-light-cone soft function or reduced soft function on the lattice (Ji et al., 2020a), as we discuss in the following. One relies on simulating HQET on the lattice, while the other requires calculating a light-meson form factor of transversely-separated current products.

The latter method has been implemented in the first exploratory lattice calculation of the reduced soft function (Zhang et al., 2020a), which includes simulations of the pion form factor in two external states with opposite large momenta, as well as the pion quasi-TMD DA. The results for the reduced soft function, which are obtained with tree-level matching and omission of lattice renormalization, are shown in Fig. 23. As one can see, they agree with the perturbative prediction for small $b_{\perp}$ within errors, as expected, and start to deviate when $b_{\perp}$ becomes large. Since the quasi-TMD DA depends on the momentum $P^{z}$, the stability of results at different $P^{z}$ suggests the validity of Eq. (250). In the future, larger statistics and improved systematics in both lattice and perturbative matching will be necessary to achieve a precision calculation of this quanity.

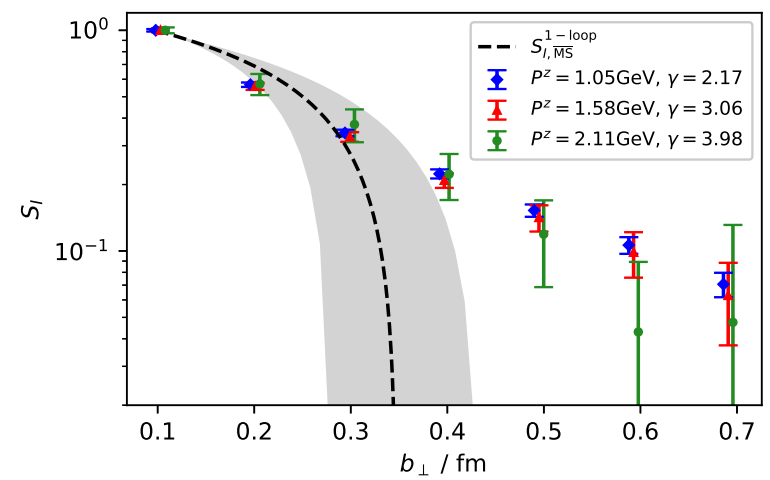

FIG. 23: The reduced soft factor as a function of $b_{\perp}$ extracted from the light-meson form factor in Sec. $\mathrm{V}$ (Zhang et al., 2020a). The results are obtained with quasi-TMD DAs at different pion momentum $P^{z}$, with perturbative matching and power corrections ignored. The dashed line is one-loop prediction in perturbation theory, which hits the Landau pole at $b_{\perp} \sim 0.3 \mathrm{fm}$, and the grey band is the error by varying $\mu$ by a factor of $1 / \sqrt{2}$ and $\sqrt{2}$. 


\section{CONCLUSION AND OUTLOOK}

Since Feynman proposed the parton model more than fifty years ago, our understanding of the partonic structure of the proton has been greatly advanced. On one hand, a number of high-energy experiments carried out at facilities worldwide including SLAC, DESY, CERN, Fermi Lab, JLab, BNL, etc. allowed us to probe various aspects of hadronic structures at different energies and polarizations. On the other hand, many parton observables have been proposed in parallel that provide a multi-dimensional description of the proton structure, including the collinear PDFs, TMDPDFs, GPDs, parton DAs, LFWFs and so on.

Although QCD factorization theorems with RG improvement allow us to extract these parton observables through their connection to experimental observables, it is highly desirable to predict them from $a b$ initio calculations such as lattice QCD. Developments along this line have been rather slow due to difficulties in simulating real-time dynamics. The situation, however, has changed since the proposal of LaMET a few years ago, which provides a systematically improvable method to calculate parton physics from first principles.

In this paper, we give an overview of LaMET formalism and its applications to observables which can be accessed in lattice QCD and other non-perturbative methods. By investigating the frame dependence of the structure of bound state hadrons, we explain how the IMF physics or parton physics naturally arises as an EFT description of the proton structure. Such an EFT description is most naturally formulated in SCET and LFQ, but practical non-perturbative calculations of the proton matrix elements have been difficult. LaMET in effect provides what is needed to realize LFQ. This is achieved by forming appropriate quasi parton observables in a large momentum state and match them to the true parton observables on the LF through factorization. In the case of PDFs, the former corresponds to finite-momentum distributions whose running is controlled by the momentum RGE, whereas the latter corresponds to IMF PDFs whose running is controlled by the usual RGE. It should be pointed out that LaMET is a very general framework which can be applied to large-momentum physical quantities calculated with any non-perturbative methods, either Euclidean (with imaginary time) or Minkowskian (with real time). Moreover, given a large momentum state, the same parton physics can be determined from different quasi observables that form a universality class.

We then present how to calculate the parton observables in practice, with a particular focus on the collinear PDFs, GPDs, DAs, TMDPDFs and LFWFs. We also discuss the proton spin structure and show how the partonic contributions to proton spin can be obtained following the same approach. We finally summarize the lattice studies carried out so far with LaMET which, on one hand, demonstrate that LaMET is a promising approach to compute partonic structures of the proton, and on the other hand, clearly indicate that a lot of improvements are still required to reach such an accuracy that the lattice results can have considerable impact on phenomenology.

We complete this review with a few comments on improvements of lattice calculations for the future. We recommend (Alexandrou et al., 2019b) for more systematic discussion on some of the issues, for example, the continuum, infinite volume, and physical pion mass limits.

- Large hadron momentum. Since the future of LaMET lies in larger momenta which naturally require smaller lattice spacings, it will be critical to address the challenges from using large momenta and small spacings for exa-scale computations, such as the excited state contamination or topological charge freezing problem.

- Renormalization. As discussed in Sec. III.D the mass renormalization of Wilson line operators is favored for it is gauge invariant and does not introduce extra higher-twist effects or large statistical errors at long distance. However, its matching to the $\overline{\mathrm{MS}}$ scheme, especially the renormalon ambiguities, still needs to be resolved for a full systematic application. Moreover, alternative schemes that include the above features are also highly desirable.

- Higher-order perturbative matching. In current LaMET calculations, one-loop perturbative matching has brought considerable corrections. Higherorder matching kernels will be necessary to control the systematics from this procedure.

- Power corrections. They are important if the hadron momentum is not very large or when $x$ is close to 0 or 1 . Little progress has been made toward a model-independent determination of the power corrections so far. One contingent strategy is to extrapolate to $P^{z} \rightarrow \infty$ limit after implementing matching and target-mass corrections, but the ultimate solution relies on the lattice calculation of higher-twist distributions that has been discussed in Sec. IV.C.

The above discussion of systematics is generic and applies to all quasi-observables. The rich theoretical developments in the past years have paved the way for calculating a wide range of parton observables using LaMET. With the rapid increase in computing resources and progress in developing new techniques and algorithms, we expect to see the above systematics to be kept under control step by step in the future. That would be important in establishing LaMET as a systematic approach to computing parton physics, and making lattice calculations play a crucial role in the EIC era. 


\section{ACKNOWLEDGMENTS}

The authors are thankful for collaborations with G. Bali, V. M. Braun, J.-W. Chen, W. Detmold, M. Ebert, X. Gao, B. Glässle, M. Göckeler, M. Gruber, Y. Hatta, F. Hutzler, T. Ishikawa, T. Izubuchi, L. Jin, N. Karthik, P. Korcyl, R. Li, C.-J. D. Lin, H.-W. Lin, K.-F. Liu, C. Monahan, S. Mukherjee, P. Petreczky, A. Schäfer, C. Schugert, P. Shanahan, I. Stewart, P. Sun, S. Syritsyn, A. Vladimirov, M. Wagman, W. Wang, P. Wein, X. Xiong, J. Xu, Y. Xu, Y.-B. Yang, F. Yuan, I. Zahed, Q.-A. Zhang, R. Zhang, S. Zhao and R. Zhu. The authors are also indebted to the enlightening discussions with J. Chang, K. T. Chao, K. Cichy, T. Cohen, J. Collins, M. Constantinou, M. Engelhardt, J. Green, Y. Jia, K. Jansen, K. Lee, J. Karpie, H.-N. Li, J. P. Ma, Y.Q. Ma, A. Manohar, R. McKeown, S. Meinel, B. Mistlberger, J. Negele, K. Orginos, J. Qiu, A. Radyushkin, E. Shuryak, G. Sterman, R. Sufian, F. Steffens, A. WalkerLoud, and C.-P. Yuan. XJ has been partially supported by the U.S. Department of Energy, Office of Science, Office of Nuclear Physics, under contract No. DE-FG0293ER-40762. YZ is supported by the U.S. Department of Energy, Office of Science, Office of Nuclear Physics, under contract No. DE-SC0012704, No. DE-AC02-06CH11357, and within the framework of the TMD Topical Collaboration. YZ is also supported by the U.S. Department of Energy, Office of Science, Office of Nuclear Physics and Office of Advanced Scientific Computing Research within the framework of Scientific Discovery through Advance Computing (ScIDAC) award Computing the Properties of Matter with Leadership Computing Resources. JHZ is supported in part by National Natural Science Foundation of China under grant No. 11975051, No. 12061131006, and by the Fundamental Research Funds for the Central Universities. YSL is supported by $\mathrm{Na}$ tional Natural Science Foundation of China under Grant No. 11905126.

\section{APPENDIX A: ACRONYMS, ABBREVIATIONS AND TERMINOLOGIES}

Here we list some acronyms, abbreviations and terminologies used throughout this review:

\begin{tabular}{|c|c|}
\hline $\mathrm{AM}$ & angular momentum \\
\hline BFKL & Balitsky-Fadin-Kuraev-Lipatov \\
\hline BPHZ & Bogoliubov-Parasiuk-Hepp-Zimmermann \\
\hline BRST & Becchi-Rouet-Stora-Tyutin \\
\hline $\mathrm{DA}$ & distribution amplitude \\
\hline DGLAP & Dokshizer-Gribov-Lipatov-Altarelli-Parisi \\
\hline DIS & deep-inelastic scattering \\
\hline DR & dimensional regularization \\
\hline DVCS & deeply-virtual Compton scattering \\
\hline DVMP & deeply-virtual meson production \\
\hline DY & Drell-Yan \\
\hline EFT & effective field theory \\
\hline EIC & Electron-Ion Collider \\
\hline EOM & equation of motion \\
\hline ERBL & Efremov-Radyushkin-Brodsky-Lepage \\
\hline GCPO & generalized collinear parton observable \\
\hline GPD & generalized parton distribution \\
\hline GTMD & $\begin{array}{l}\text { generalized transverse-momentum- } \\
\text { dependent distribution }\end{array}$ \\
\hline HQET & heavy-quark effective theory \\
\hline IMF & infinite-momentum frame \\
\hline $\mathrm{IR}$ & infrared \\
\hline LaMET & large momentum effective theory \\
\hline $\mathrm{LC}$ & light-cone \\
\hline $\mathrm{LF}$ & light-front \\
\hline LFWF & light-front wave function \\
\hline$\overline{\mathrm{MS}}$ & modified minimal subtraction \\
\hline NPR & non-perturbative renormalization \\
\hline OAM & orbital angular momentum \\
\hline OPE & operator product expansion \\
\hline $\mathrm{PDF}$ & parton distribution function \\
\hline QCD & quantum chromodynamics \\
\hline QED & quantum electrodynamics \\
\hline QFT & quantum field theory \\
\hline RGE & renormalization group equation \\
\hline $\mathrm{RI} / \mathrm{MOM}$ & $\begin{array}{l}\text { regularization-independent } \\
\text { momentum subtraction }\end{array}$ \\
\hline SCET & soft-collinear effective theory \\
\hline SIDIS & semi-inclusive deep-inelastic scattering \\
\hline TMD & transverse-momentum-dependent \\
\hline UV & ultraviolet \\
\hline
\end{tabular}

Parton model: a model proposed by R. Feynman in which hadrons are viewed as a collection of pointlike quasi-free partons.

Parton distribution function: a probability function describing how the longitudinal momentum is distributed among the partons (quarks and gluons) in a hadron.

Factorization theorem: a theorem that separates hadronic observables into process-dependent short- 
distance partonic observables and universal longdistance functions characterizing the hadron structure.

Light-front quantization: a quantization program that is carried out at equal light-front time and yields a relativistic description of QCD bound states in terms of light-front wave functions.

Bjorken $x_{B}$ : The variable proposed by J. D. Bjorken to characterize the kinematics in DIS. Its definition is given above Eq. (11).

Scaling: The behavior that an observable is independent of the scale at which it is probed.

Effective field theory: a theory framework that describes physical phenomena at a given length scale using only active degrees of freedom at that scale, while integrating out degrees of freedom at other length scales.

Renormalization group equation: an equation that describes how a physical system can be viewed and interpreted at different scales.

HQET: an effective field theory obtained from QCD by taking the infinite heavy quark mass limit.

Gauge link or Wilson line: a nonlocal quantity constructed as exponentials of integrals of gauge fields along a given path, used to connect fields at different spacetime points to maintain gauge invariance.

Compton amplitude: the quantum amplitude for scattering of a (virtual) photon by the proton.

Auxiliary field approach: an approach in which the nonlocal gauge link can be replaced by the twopoint function of the auxiliary field.

Matching: a procedure used to relate full theory operators to effective field theory operators.

Nonsinglet: a combination accounting for the difference between quark distributions, e.g., the isovector combination $u-d$ discussed extensively in the context of this review.

Universality class: a collection of operators that flow into the same fixed point under momentum renormalization group running.

Quasi-light-front correlations: spatial correlations defining the finite momentum distributions.

Collinear divergence: divergence in a Feynman diagram when loop momentum of the internal line is collinear to that of the external massless particle.

Two-particle-irreducible diagram: a Feynman diagram that cannot be divided into disconnected parts by cutting two internal lines.

Wilson fermion: a way to discretize the QCD fermion action on the lattice, which breaks down the chiral symmetry.
Generalized parton distribution: generalization of PDFs to non-forward kinematics, i.e., the initial and final states have different momenta.

Skewness: defined to characterize the longitudinal momentum transfer in GPDs.

Distribution amplitude: transition matrix element between vacuum and hadron state, representing the probability amplitude of finding a given Fock state in the hadron.

Twist: defined as dimension - spin of the operator. Leading-twist (higher-twist) denotes the leading (nonleading) power behavior in the quantity under investigation.

Zero-mode: the degrees of freedom with zero longitudinal momentum in LFQ.

Transverse momentum dependent(TMD) PDF: defined in Eq. (220), the distribution function of both longitudinal and transverse momentum for partons.

Staple-shaped gauge-link: the pair of gauge-links separated along transverse directions that appear in the definition of TMD-PDFs, they are defined in Eq. (166), Eq. (173) and Eq. (196).

Rapidity divergence: the divergence of TMDPDF and soft functions due to the presence of infinite rapidity scale introduced by the infinite-long gaugelinks.

Light-cone regulator: regulators that regulates the rapidity divergence. First appeared in the paragraph of Eq. (168).

On-light-cone: rapidity regulator that maintain the presence of light-like separations in the gauge-link.

Off-light-cone: rapidity regulator that makes the separations of the gauge-link non-light-like.

Soft function: functions that capture the factorable soft radiations of TMDPDF. Defined in Eq. (172) for on-light-cone and Eq. (202) for off-light-cone.

Collins Soper kernel: the kernel for rapidity evolution of TMDPDF, see Eq. (191).

Quasi-TMDPDF: defined in Eq. (195), similar to TMDPDF but with light-like separations replaced by space-like ones.

Pinch-pole singularity: the divergence due to infinite long gauge-link pair in the quasi-TMDPDF. Can be subtracted out by the factor $Z_{E}$, see discussion below Eq. (198).

Off-light-cone soft function: soft function using offlight-cone regulator. Defined in Eq. (202) and Eq. (224). Required for matching quasi-TMDPDF to TMDPDF.

Reduced soft function: the rapidity independent part of off-light-cone soft function, see Eq. (204). 
Light-Front wave function: the wave function for hadron state in light-front quantization, expanded in the Free -Fock state.

Reduced diagram: the diagram showing the powerleading region of IR divergences. All the IR safe propagators are shrunk to blobs.

Momentum smearing: a lattice technique to increase the overlap of the field and nonzeromomentum state.

Non-singlet: transforms under the fundmental representation of $S U\left(N_{f}\right)$ with $N_{f}$ the quark flavor number.

\section{APPENDIX B: CONVENTIONS}

We use the following convention for the metric tensor

$$
g^{\mu \nu}=\operatorname{diag}(1,-1,-1,-1) .
$$

In ordinary coordinates, a generic four-vector is denoted as $v^{\mu}=\left(v^{0}, v^{x}, v^{y}, v^{z}\right)$ or $v^{\mu}=\left(v^{0}, \vec{v}_{\perp}, v^{z}\right)$. For example, the spacelike and timelike direction vector are written as $n_{z}=(0,0,0,1)$ and $n_{t}=(1,0,0,0)$, respectively. In light-cone coordinates $\xi^{ \pm}=\frac{1}{\sqrt{2}}\left(\xi^{0} \pm \xi^{3}\right)$, a vector is denoted as $v^{\mu}=\left(v^{+}, v^{-}, \vec{v}_{\perp}\right)$.

The hadron state $|P\rangle$ is normalized as

$$
\left\langle P^{\prime} \mid P\right\rangle=(2 \pi)^{3} 2 P^{0} \delta^{(3)}\left(\vec{P}-\vec{P}^{\prime}\right) .
$$

The covariant derivative and the Wilson line gauge link in the fundamental representation are defined as

$$
D^{\mu} \psi=\left(\partial^{\mu}+i g A^{\mu}\right) \psi=\left(\partial^{\mu}+i g t^{a} A_{a}^{\mu}\right) \psi
$$

and

$$
\begin{aligned}
& W\left(x_{2}, x_{1}\right)= \\
& \exp \left[-i g \int_{0}^{1} d t\left(x_{2}-x_{1}\right)_{\mu} A^{\mu}\left(x_{1}+\left(x_{2}-x_{1}\right) t\right)\right] .
\end{aligned}
$$

The ones in the adjoint representation are completely analogous.

We use $O_{\Gamma}(s)$ to generically denote an operator defining the corresponding (quasi) parton observable, where $s$ can be a lightlike (for parton observables) or spacelike (for quasi parton observables) separation, and $\Gamma$ is a Dirac structure. The momentum fraction in a quasiobservable is denoted as $y$, while that in the usual parton observable is denoted as $x$.

The lightcone operator that defines the quark parton observable is

$$
O_{\Gamma}(\lambda n)=\bar{\psi}(0) \Gamma W(0, \lambda n) \psi(\lambda n)
$$

with $\Gamma$ denoting a Dirac matrix. If we take $\Gamma=\not \equiv \equiv \gamma^{+}$, the unpolarized quark PDF is then given by

$$
q(x)=\frac{1}{2 P^{+}} \int \frac{d \lambda}{2 \pi} e^{i x \lambda}\left\langle P\left|O_{\gamma^{+}}(\lambda n)\right| P\right\rangle
$$

with $n^{\mu}=1 / \sqrt{2}\left(1 / P^{+}, 0,0,-1 / P^{+}\right)$.

Accordingly, the quark quasi-observable is defined by

$$
O_{\Gamma}(z)=\bar{\psi}\left(z n_{z} / 2\right) \Gamma W\left(z n_{z} / 2,-z n_{z} / 2\right) \psi\left(-z n_{z} / 2\right) .
$$

If we choose $\Gamma=\gamma^{t}$, the unpolarized quark quasi-PDF is then defined as

$$
\tilde{q}(y)=\frac{1}{2 P^{0}} \int \frac{d \lambda}{2 \pi} e^{i y \lambda}\left\langle P\left|O_{\gamma^{t}}(z)\right| P\right\rangle
$$

with the quasi light-cone distance $\lambda=z P^{z}$.

The staple-shaped gauge link required for the TMDPDFs is defined as:

$$
\mathcal{W}_{n}\left(\lambda n / 2+\vec{b}_{\perp}\right)=W_{n}^{\dagger}\left(\lambda n / 2+\vec{b}_{\perp}\right) W_{\perp} W_{n}(-\lambda n / 2),
$$

where

$$
W_{n}(\xi)=W(\xi+\infty n, \xi) .
$$

The un-subtracted unpolarized quark TMDPDF is then defined as:

$$
\begin{aligned}
& f\left(x, \vec{k}_{\perp}, \mu, \delta^{-} / P^{+}\right)=\frac{1}{2 P^{+}} \int \frac{d \lambda}{2 \pi} \frac{d^{2} \vec{b}_{\perp}}{(2 \pi)^{2}} e^{-i \lambda x+i \vec{k}_{\perp} \cdot \vec{b}_{\perp}} \\
& \times\left\langle P\left|\bar{\psi}\left(\lambda n / 2+\vec{b}_{\perp}\right) \not \mathcal{W}_{n}\left(\lambda n / 2+\vec{b}_{\perp}\right)\right|_{\delta^{-}} \psi(-\lambda n / 2) \mid P\right\rangle,
\end{aligned}
$$

and the TMD soft function for DY process is defined as:

$$
\begin{aligned}
& S\left(b_{\perp}, \mu, \delta^{+}, \delta^{-}\right) \\
& =\frac{\operatorname{Tr}\left\langle\left.\left.\left. 0\left|\overline{\mathcal{T}} W_{p}\left(\vec{b}_{\perp}\right)\right|_{\delta^{+}} W_{n}^{\dagger}\left(\vec{b}_{\perp}\right)\right|_{\delta^{-}} \mathcal{T} W_{n}(0)\right|_{\delta^{-}} W_{p}^{\dagger}(0)\right|_{\delta^{+}} \mid 0\right\rangle}{N_{c}} \\
& =\frac{\operatorname{Tr}\left\langle\left. 0\left|\mathcal{W}_{n}\left(\vec{b}_{\perp}\right)\right|_{\delta^{+}} \mathcal{W}_{p}^{\dagger}\left(\vec{b}_{\perp}\right)\right|_{\delta^{-}} \mid 0\right\rangle}{N_{c}},
\end{aligned}
$$

where $\left.\right|_{\delta^{ \pm}}$denotes the rapidity regulator for the gauge links involved. In terms of these, the physical scheme independent TMDPDF is defiend as:

$$
f^{\mathrm{TMD}}\left(x, b_{\perp}, \mu, \zeta\right)=\lim _{\delta^{-} \rightarrow 0} \frac{f\left(x, b_{\perp}, \mu, \delta^{-} / P^{+}\right)}{\sqrt{S\left(b_{\perp}, \mu, \delta^{-} e^{2 y_{n}}, \delta^{-}\right)}},
$$

where $\zeta \equiv 2\left(x P^{+}\right)^{2} e^{2 y_{n}}$ is the rapidity scale.

The staple-shaped gauge link for the quasi-TMDPDF is defined as:

$$
\mathcal{W}_{z}\left(\frac{\lambda n_{z}}{2}+\vec{b}_{\perp} ; L\right)=W_{z}^{\dagger}(\xi ; L) W_{\perp} W_{z}\left(-\xi^{z} n_{z} ; L\right),
$$


where

$$
W_{z}(\xi)=W\left(\xi+\left(L-\xi^{z}\right) n_{z}, \xi\right)
$$

The quasi-TMDPDF is then defined using $\mathcal{W}_{z}\left(\frac{\lambda n_{z}}{2}+\right.$ $\left.\vec{b}_{\perp} ; L\right)$ in exactly the same way as that for the unsubtracted TMDPDF:

$$
\begin{aligned}
& \tilde{f}\left(\lambda, b_{\perp}, \mu, \zeta_{z}\right)= \\
& \lim _{L \rightarrow \infty} \frac{\left\langle P\left|\bar{\psi}\left(\frac{\lambda n_{z}}{2}+\vec{b}_{\perp}\right) \gamma^{z} \mathcal{W}_{z}\left(\frac{\lambda n_{z}}{2}+\vec{b}_{\perp} ; L\right) \psi\left(-\frac{\lambda n_{z}}{2}\right)\right| P\right\rangle}{\sqrt{Z_{E}\left(2 L, b_{\perp}, \mu\right)}}
\end{aligned}
$$

where $Z_{E}\left(2 L, b_{\perp}, \mu\right)$ is a flat rectangular Euclidean Wilson-loop along the $n_{z}$ direction with length $2 L$ and width $b_{\perp}$ :

$$
Z_{E}\left(2 L, b_{\perp}, \mu\right)=\frac{1}{N_{c}} \operatorname{Tr}\left\langle 0\left|W_{\perp} \mathcal{W}_{z}\left(\vec{b}_{\perp} ; 2 L\right)\right| 0\right\rangle .
$$

The staple-shaped operators for LFWFs and quasiLFWFs are the same as those for TMD-PDFs and quasiTMDPDFs, and can be found in Sec. V.D

\section{REFERENCES}

Abada, A, Philippe Boucaud, G. Herdoiza, J. P. Leroy, J. Micheli, O. Pene, and J. Rodriguez-Quintero (2001), "Preliminaries on a lattice analysis of the pion light cone wave function: A Partonic signal?" Phys. Rev. D64, 074511, arXiv:hep-ph/0105221 [hep-ph].

Abdel-Rehim, A, et al. (2015), "Nucleon and pion structure with lattice QCD simulations at physical value of the pion mass," Phys. Rev. D92 (11), 114513 [Erratum: Phys. Rev.D93,no.3,039904(2016)], arXiv:1507.04936 [hep-lat].

Accardi, A, L. T. Brady, W. Melnitchouk, J. F. Owens, and N. Sato (2016a), "Constraints on large- $x$ parton distributions from new weak boson production and deepinelastic scattering data," Phys. Rev. D93 (11), 114017 , arXiv:1602.03154 [hep-ph]

Accardi, A, et al. (2016b), "Electron Ion Collider: The Next QCD Frontier," Eur. Phys. J. A52 (9), 268, arXiv:1212.1701 [nucl-ex]

Adamczyk, L, et al. (STAR) (2016), "Measurement of the transverse single-spin asymmetry in $p^{\uparrow}+p \rightarrow$ $W^{ \pm} / Z^{0}$ at RHIC," Phys. Rev. Lett. 116 (13), 132301, arXiv:1511.06003 [nucl-ex]

Agaev, S S, V. M. Braun, N. Offen, and F. A. Porkert (2012), "BELLE Data on the $\pi^{0} \gamma * \gamma$ Form Factor: A Game Changer?" Phys. Rev. D86, 077504, arXiv:1206.3968 [hep-ph].

Aghasyan, M, et al. (COMPASS) (2017), "First measurement of transverse-spin-dependent azimuthal asymmetries in the Drell-Yan process," Phys. Rev. Lett. 119 (11), 112002 arXiv:1704.00488 [hep-ex]

Aglietti, U (1994), "Consistency and lattice renormalization of the effective theory for heavy quarks," Nucl. Phys. B421, 191-216, arXiv:hep-ph/9304274 [hep-ph]

Aglietti, U, Marco Ciuchini, G. Corbo, E. Franco, G. Martinelli, and L. Silvestrini (1998), "Model independent determination of the light cone wave functions for exclusive processes," Phys. Lett. B441, 371-375, arXiv:hep-ph/9806277 [hep-ph].

Aidala, Christine A, Steven D. Bass, Delia Hasch, and Gerhard K. Mallot (2013), "The Spin Structure of the Nucleon," Rev. Mod. Phys. 85, 655-691, arXiv:1209.2803 [hep-ph]

Alekhin, S, J. Blümlein, S. Moch, and R. Placakyte (2017), "Parton distribution functions, $\alpha_{s}$, and heavy-quark masses for LHC Run II," Phys. Rev. D96 (1), 014011 , arXiv:1701.05838 [hep-ph]

Alexandrou, C, S. Bacchio, M. Constantinou, J. Finkenrath, K. Hadjiyiannakou, K. Jansen, G. Koutsou, and A. Vaquero Aviles-Casco (2019a), "The nucleon axial, tensor and scalar charges and $\sigma$-terms in lattice QCD," arXiv:1909.00485 [hep-lat]

Alexandrou, C, M. Constantinou, K. Hadjiyiannakou, K. Jansen, C. Kallidonis, G. Koutsou, A. Vaquero Avilés-Casco, and C. Wiese (2017a), "Nucleon Spin and Momentum Decomposition Using Lattice QCD Simulations," Phys. Rev. Lett. 119 (14), 142002, arXiv:1706.02973 [hep-lat].

Alexandrou, C, M. Constantinou, K. Hadjiyiannakou, K. Jansen, and F. Manigrasso (2020a), "Flavor decomposition for the proton helicity parton distribution functions," arXiv:2009.13061 [hep-lat] 
Alexandrou, C, et al. (2020b), "Moments of nucleon generalized parton distributions from lattice QCD simulations at physical pion mass," Phys. Rev. D101 (3), 034519. arXiv:1908.10706 [hep-lat]

Alexandrou, Constantia, Krzysztof Cichy, Martha Constantinou, Jeremy R. Green, Kyriakos Hadjiyiannakou, Karl Jansen, Floriano Manigrasso, Aurora Scapellato, and Fernanda Steffens (2020c), "Lattice continuum-limit study of nucleon quasi-PDFs," arXiv:2011.00964 [hep-lat]

Alexandrou, Constantia, Krzysztof Cichy, Martha Constantinou, Kyriakos Hadjiyiannakou, Karl Jansen, Haralambos Panagopoulos, and Fernanda Steffens (2017b), "A complete non-perturbative renormalization prescription for quasi-PDFs," Nucl. Phys. B923, 394-415. arXiv:1706.00265 [hep-lat]

Alexandrou, Constantia, Krzysztof Cichy, Martha Constantinou, Kyriakos Hadjiyiannakou, Karl Jansen, Aurora Scapellato, and Fernanda Steffens (2019b), "Systematic uncertainties in parton distribution functions from lattice QCD simulations at the physical point," Phys. Rev. D99 (11), 114504, arXiv:1902.00587 [hep-lat]

Alexandrou, Constantia, Krzysztof Cichy, Martha Constantinou, Kyriakos Hadjiyiannakou, Karl Jansen, Aurora Scapellato, and Fernanda Steffens (2020d), "Unpolarized and helicity generalized parton distributions of the proton within lattice QCD," arXiv:2008.10573 [hep-lat].

Alexandrou, Constantia, Krzysztof Cichy, Martha Constantinou, Karl Jansen, Aurora Scapellato, and Fernanda Steffens (2018a), "Light-Cone Parton Distribution Functions from Lattice QCD," Phys. Rev. Lett. 121 (11), 112001, arXiv:1803.02685 [hep-lat]

Alexandrou, Constantia, Krzysztof Cichy, Martha Constantinou, Karl Jansen, Aurora Scapellato, and Fernanda Steffens (2018b), "Transversity parton distribution functions from lattice QCD," Phys. Rev. D98 (9), 091503. arXiv:1807.00232 [hep-lat]

Alexandrou, Constantia, Krzysztof Cichy, Vincent Drach, Elena Garcia-Ramos, Kyriakos Hadjiyiannakou, Karl Jansen, Fernanda Steffens, and Christian Wiese (2015), "Lattice calculation of parton distributions," Phys. Rev. D92, 014502, arXiv:1504.07455 [hep-lat]

Altarelli, Guido, and G. Parisi (1977), "Asymptotic Freedom in Parton Language," Nucl. Phys. B126, 298-318.

Aoki, S, et al. (Flavour Lattice Averaging Group) (2020), "FLAG Review 2019," Eur. Phys. J. C80 (2), 113 arXiv:1902.08191 [hep-lat]

Aoki, Yasumichi, Tom Blum, Huey-Wen Lin, Shigemi Ohta, Shoichi Sasaki, Robert Tweedie, James Zanotti, and Takeshi Yamazaki (2010), "Nucleon isovector structure functions in $(2+1)$-flavor QCD with domain wall fermions," Phys. Rev. D82, 014501, arXiv:1003.3387 [hep-lat].

Aprahamian, Ani, et al. (2015), "Reaching for the horizon: The 2015 long range plan for nuclear science,"

Arefeva, IYa (1980), "QUANTUM CONTOUR FIELD EQUATIONS," Phys. Lett. B 93, 347-353.

Aubert, Bernard, et al. (BaBar) (2009), "Measurement of the gamma gamma* $\longrightarrow$ pi0 transition form factor," Phys. Rev. D80, 052002, arXiv:0905.4778 [hep-ex].

Bacchetta, Alessandro, Valerio Bertone, Chiara Bissolotti, Giuseppe Bozzi, Filippo Delcarro, Fulvio Piacenza, and Marco Radici (2019), "Transverse-momentum-dependent parton distributions up to $\mathrm{N}^{3} \mathrm{LL}$ from Drell-Yan data," arXiv:1912.07550 [hep-ph].

Bacchetta, Alessandro, Filippo Delcarro, Cristian Pisano,
Marco Radici, and Andrea Signori (2017), "Extraction of partonic transverse momentum distributions from semi-inclusive deep-inelastic scattering, DrellYan and Z-boson production," JHEP 06, 081, [Erratum: JHEP06,051(2019)], arXiv:1703.10157 [hep-ph].

Bagan, E, and P. Gosdzinsky (1994), "Renormalizability of the heavy quark effective theory," $\quad$ Phys. Lett. B320, 123-129, arXiv:hep-ph/9305297 [hep-ph].

Baikov, PA, K.G. Chetyrkin, A.V. Smirnov, V.A. Smirnov, and M. Steinhauser (2009), "Quark and gluon form factors to three loops," Phys. Rev. Lett. 102, 212002, arXiv:0902.3519 [hep-ph],

Bali, Gunnar S, Vladimir M. Braun, Simon Bürger, Meinulf Göckeler, Michael Gruber, Fabian Hutzler, Piotr Korcyl, Andreas Schäfer, André Sternbeck, and Philipp Wein (2019), "Light-cone distribution amplitudes of pseudoscalar mesons from lattice QCD," JHEP 08, 065 , arXiv:1903.08038 [hep-lat]

Bali, Gunnar S, Vladimir M. Braun, Benjamin Gläßle, Meinulf Göckeler, Michael Gruber, Fabian Hutzler, Piotr Korcyl, Andreas Schäfer, Philipp Wein, and JianHui Zhang (2018a), "Pion distribution amplitude from Euclidean correlation functions: Exploring universality and higher-twist effects," Phys. Rev. D98 (9), 094507. arXiv:1807.06671 [hep-lat].

Bali, Gunnar S, Sara Collins, Benjamin Gläßle, Meinulf Göckeler, Johannes Najjar, Rudolf H. Rödl, Andreas Schäfer, Rainer W. Schiel, André Sternbeck, and Wolfgang Söldner (2014), "The moment $\langle x\rangle_{u-d}$ of the nucleon from $N_{f}=2$ lattice QCD down to nearly physical quark masses," Phys. Rev. D90 (7), 074510, arXiv:1408.6850 [hep-lat].

Bali, Gunnar S, Bernhard Lang, Bernhard U. Musch, and Andreas Schäfer (2016), "Novel quark smearing for hadrons with high momenta in lattice QCD," Phys. Rev. D93 (9), 094515, arXiv:1602.05525 [hep-lat].

Bali, Gunnar S, et al. (2018b), "Pion distribution amplitude from Euclidean correlation functions," Proceedings, 35th International Symposium on Lattice Field Theory (Lattice 2017): Granada, Spain, June 18-24, 2017, Eur. Phys. J. C78 (3), 217 , arXiv:1709.04325 [hep-lat].

Balitsky, I (1996), "Operator expansion for highenergy scattering," Nucl. Phys. B463, 99-160. arXiv:hep-ph/9509348 [hep-ph].

Balitsky, I I, Vladimir M. Braun, Y. Koike, and K. Tanaka (1996), " $\mathrm{Q}^{* *} 2$ evolution of chiral odd twist - three distributions $\mathrm{h}(\mathrm{L})\left(\mathrm{x}, \mathrm{Q}^{* *} 2\right)$ and e $\left(\mathrm{x}, \mathrm{Q}^{* *} 2\right)$ in the large N(c) limit," Phys. Rev. Lett. 77, 3078-3081, arXiv:hep-ph/9605439 [hep-ph].

Balitsky, I I, and L. N. Lipatov (1978), "The Pomeranchuk Singularity in Quantum Chromodynamics," Sov. J. Nucl. Phys. 28, 822-829, [Yad. Fiz.28,1597(1978)].

Balitsky, Ian, Wayne Morris, and Anatoly Radyushkin (2019), "Gluon Pseudo-Distributions at Short Distances: Forward Case," arXiv:1910.13963 [hep-ph].

Balitsky, II, and Vladimir M. Braun (1989), "Evolution Equations for QCD String Operators," Nucl. Phys. B 311, 541-584

Ball, Patricia, V. M. Braun, and A. Lenz (2006), "Highertwist distribution amplitudes of the K meson in QCD," JHEP 05, 004, arXiv:hep-ph/0603063 [hep-ph].

Ball, Richard D, et al. (NNPDF) (2017), "Parton distributions from high-precision collider data," Eur. Phys. J. C77 (10), 663 arXiv:1706.00428 [hep-ph]. 
Barone, Vincenzo, Alessandro Drago, and Philip G. Ratcliffe (2002), "Transverse polarisation of quarks in hadrons," Phys. Rept. 359, 1-168, arXiv:hep-ph/0104283 [hep-ph]

Bars, I, and Michael B. Green (1978), "Poincare and Gauge Invariant Two-Dimensional QCD," Phys. Rev. D17, 537

Bass, Steven D (2005), "The Spin structure of the proton," Rev. Mod. Phys. 77, 1257-1302, arXiv:hep-ph/0411005

Bauer, Christian W, Sean Fleming, Dan Pirjol, and Iain W. Stewart (2001), "An Effective field theory for collinear and soft gluons: Heavy to light decays," Phys. Rev. D63, 114020, arXiv:hep-ph/0011336 [hep-ph].

Bauer, Christian W, Dan Pirjol, and Iain W. Stewart (2002), "Soft collinear factorization in effective field theory," Phys. Rev. D65, 054022, arXiv:hep-ph/0109045 [hep-ph]

Bauer, Christian W, and Iain W. Stewart (2001), "Invariant operators in collinear effective theory," $\quad$ Phys. Lett. B516, 134-142 arXiv:hep-ph/0107001 [hep-ph]

Becher, Thomas, and Matthias Neubert (2011), "Drell-Yan Production at Small $q_{T}$, Transverse Parton Distributions and the Collinear Anomaly," Eur. Phys. J. C71, 1665 arXiv:1007.4005 [hep-ph]

Belitsky, A V, and A. V. Radyushkin (2005), "Unraveling hadron structure with generalized parton distributions," Phys. Rept. 418, 1-387, arXiv:hep-ph/0504030 [hep-ph],

Belitsky, Andrei V, X. Ji, and F. Yuan (2003), "Final state interactions and gauge invariant parton distributions," Nucl. Phys. B656, 165-198 arXiv:hep-ph/0208038 [hep-ph]

Belitsky, Andrei V, Xiang-dong Ji, and Feng Yuan (2004), "Quark imaging in the proton via quantum phase space distributions," Phys. Rev. D69, 074014. arXiv:hep-ph/0307383 [hep-ph]

Beneke, M (1999), "Renormalons," Phys. Rept. 317, 1-142, arXiv:hep-ph/9807443 [hep-ph]

Beneke, M, and Vladimir M. Braun (1994), "Heavy quark effective theory beyond perturbation theory: Renormalons, the pole mass and the residual mass term," Nucl. Phys. B426, 301-343. arXiv:hep-ph/9402364 [hep-ph]

Beneke, M, and Vladimir M. Braun (1995), "Power corrections and renormalons in DrellYan production," Nucl. Phys. B454, 253-290. arXiv:hep-ph/9506452 [hep-ph]

Beneke, M, and Vladimir M. Braun (2000), "Renormalons and power corrections," , 1719-1773 arXiv:hep-ph/0010208 [hep-ph]

Beneke, M, Vladimir M. Braun, and Lorenzo Magnea (1997), "Phenomenology of power corrections in fragmentation processes in e+ e- annihilation," Nucl. Phys. B497, 297-333 arXiv:hep-ph/9701309 [hep-ph]

Bertone, Valerio, Ignazio Scimemi, and Alexey Vladimirov (2019), "Extraction of unpolarized quark transverse momentum dependent parton distributions from Drell-Yan/Z-boson production," JHEP 06, 028, arXiv:1902.08474 [hep-ph]

Bhat, Manjunath, Krzysztof Cichy, Martha Constantinou, and Aurora Scapellato (2020), "Parton distribution functions from lattice QCD at physical quark masses via the pseudo-distribution approach," arXiv:2005.02102 [hep-lat].

Bhattacharya, Shohini, Krzysztof Cichy, Martha Constantinou, Andreas Metz, Aurora Scapellato, and Fernanda Steffens (2020a), "New insights on proton structure from lattice QCD: the twist-3 parton distribution function $g_{T}(x)$,"
arXiv:2004.04130 [hep-lat]

Bhattacharya, Shohini, Krzysztof Cichy, Martha Constantinou, Andreas Metz, Aurora Scapellato, and Fernanda Steffens (2020b), "One-loop matching for the twist-3 parton distribution $g_{T}(x)$," Phys. Rev. D $102(3), 034005$. arXiv:2005.10939 [hep-ph]

Bhattacharya, Shohini, Krzysztof Cichy, Martha Constantinou, Andreas Metz, Aurora Scapellato, and Fernanda Steffens (2020c), "The role of zero-mode contributions in the matching for the twist-3 PDFs $e(x)$ and $h_{L}(x)$," arXiv:2006.12347 [hep-ph].

Bhattacharya, Shohini, Christopher Cocuzza, and Andreas Metz (2019a), "Exploring twist-2 GPDs through quasi-distributions in a diquark spectator model," arXiv:1903.05721 [hep-ph].

Bhattacharya, Shohini, Christopher Cocuzza, and Andreas Metz (2019b), "Generalized quasi parton distributions in a diquark spectator model," Phys. Lett. B788, 453-463, arXiv:1808.01437 [hep-ph].

Bhattacharya, Shohini, Andreas Metz, Vikash Kumar Ojha, Jeng-Yuan Tsai, and Jian Zhou (2018), "Exclusive double quarkonium production and generalized TMDs of gluons," arXiv:1802.10550 [hep-ph]

Bhattacharya, Shohini, Andreas Metz, and Jian Zhou (2017), "Generalized TMDs and the exclusive double Drell-Yan process," Phys. Lett. B771, 396-400. arXiv:1702.04387 [hep-ph]

Bigi, Ikaros I Y, Mikhail A. Shifman, N. G. Uraltsev, and A. I. Vainshtein (1994), "The Pole mass of the heavy quark. Perturbation theory and beyond," Phys. Rev. D50, 2234-2246. arXiv:hep-ph/9402360 [hep-ph].

Bjorken, J D, and Emmanuel A. Paschos (1969), "Inelastic Electron Proton and gamma Proton Scattering, and the Structure of the Nucleon," Phys. Rev. 185, 1975-1982

Bloom, Elliott D, et al. (1969), "High-Energy Inelastic e p Scattering at 6-Degrees and 10-Degrees," Phys. Rev. Lett. 23, 930-934.

Bodwin, Geoffrey T (1985), "Factorization of the Drell-Yan Cross-Section in Perturbation Theory," Phys. Rev. D31, 2616, [Erratum: Phys. Rev.D34,3932(1986)].

Boer, Daniel, and P. J. Mulders (1998), "Time reversal odd distribution functions in leptoproduction," $\quad$ Phys. Rev. D57, 5780-5786 arXiv:hep-ph/9711485 [hep-ph].

Boer, Daniel, et al. (2011), "Gluons and the quark sea at high energies: Distributions, polarization, tomography," arXiv:1108.1713 [nucl-th].

Braun, V, P. Gornicki, and L. Mankiewicz (1995), "Ioffe - time distributions instead of parton momentum distributions in description of deep inelastic scattering," Phys. Rev. D 51, 6036-6051, arXiv:hep-ph/9410318

Braun, V, and Dieter Müller (2008), "Exclusive processes in position space and the pion distribution amplitude," Eur. Phys. J. C55, 349-361, arXiv:0709.1348 [hep-ph].

Braun, V M (2006), "Nucleons on the light-cone: Theory and phenomenology of baryon distribution amplitudes," in Continuous advances in QCD. Proceedings, 7th Workshop, QCD 2006, pp. 42-57, arXiv:hep-ph/0608231 [hep-ph]

Braun, V M, S. Collins, M. Göckeler, P. Pérez-Rubio, A. Schäfer, R. W. Schiel, and A. Sternbeck (2015), "Second Moment of the Pion Light-cone Distribution Amplitude from Lattice QCD," Phys. Rev. D92 (1), 014504. 
arXiv:1503.03656 [hep-lat]

Braun, V M, G. P. Korchemsky, and Dieter Müller (2003), "The Uses of conformal symmetry in QCD," Prog. Part. Nucl. Phys. 51, 311-398 arXiv:hep-ph/0306057 [hep-ph]

Braun, V M, T. Lautenschlager, A. N. Manashov, and B. Pirnay (2011), "Higher twist parton distributions from light-cone wave functions," Phys. Rev. D83, 094023 arXiv:1103.1269 [hep-ph]

Braun, Vladimir M (1995), "QCD renormalons and higher twist effects," in '95 QCD and high-energy hadronic interactions. Proceedings, 30th Rencontres de Moriond, Moriond Particle Physics Meetings, Hadronic Session, Le Arcs, France, March 19-25, 1995, pp. 271-278, arXiv:hep-ph/9505317 [hep-ph]

Braun, Vladimir M, and I. E. Filyanov (1990), "Conformal Invariance and Pion Wave Functions of Nonleading Twist," Z. Phys. C48, 239-248, [Sov. J. Nucl. Phys.52,126(1990); Yad. Fiz.52,199(1990)].

Braun, Vladimir M, Einan Gardi, and Stefan Gottwald (2004), "Renormalon approach to higher twist distribution amplitudes and the convergence of the conformal expansion," Nucl. Phys. B685, 171-226 arXiv:hep-ph/0401158 [hep-ph]

Braun, Vladimir M, Alexey Vladimirov, and Jian-Hui Zhang (2019), "Power corrections and renormalons in parton quasidistributions," Phys. Rev. D99 (1), 014013. arXiv:1810.00048 [hep-ph]

Braun, VM, K.G. Chetyrkin, and B.A. Kniehl (2020), "Renormalization of parton quasi-distributions beyond the leading order: spacelike vs. timelike," arXiv:2004.01043 [hep-ph]

Briceño, Raúl A, Maxwell T. Hansen, and Christopher J. Monahan (2017), "Role of the Euclidean signature in lattice calculations of quasidistributions and other nonlocal matrix elements," Phys. Rev. D 96 (1), 014502 , arXiv:1703.06072 [hep-lat]

Briceno, Raul A, Jozef J. Dudek, and Ross D. Young (2018), "Scattering processes and resonances from lattice QCD," Rev. Mod. Phys. 90 (2), 025001. arXiv:1706.06223 [hep-lat]

Briceño, Raúl A, Juan V. Guerrero, Maxwell T. Hansen, and Christopher J. Monahan (2018), "Finitevolume effects due to spatially nonlocal operators," Phys. Rev. D98 (1), 014511, arXiv:1805.01034 [hep-lat].

Bringewatt, J, N. Sato, W. Melnitchouk, Jian-Wei Qiu, F. Steffens, and M. Constantinou (2020), "Confronting lattice parton distributions with global QCD analysis," arXiv:2010.00548 [hep-ph]

Broadhurst, David J, and A. G. Grozin (1991), "Two loop renormalization of the effective field theory of a static quark," Phys. Lett. B267, 105-110, arXiv:hep-ph/9908362 [hep-ph]

Brodsky, SJ (2002), "Perspectives on exclusive processes in QCD," in Exclusive Processes at High Momentum Transfer, pp. 1-33, arXiv:hep-ph/0208158.

Brodsky, Stanley J, and G. Peter Lepage (1989), "Exclusive Processes in Quantum Chromodynamics," IN *MUELLER, A.H. (ED.): PERTURBATIVE QUANTUM CHROMODYNAMICS* 93-240 AND SLAC STANFORD - SLAC-PUB-4947 (89,REC.JUL.) 149p, Adv. Ser. Direct. High Energy Phys. 5, 93-240

Brodsky, Stanley J, Hans-Christian Pauli, and Stephen S.
Pinsky (1998), "Quantum chromodynamics and other field theories on the light cone," Phys. Rept. 301, 299-486, arXiv:hep-ph/9705477 [hep-ph].

Broniowski, Wojciech, and Enrique Ruiz Arriola (2017), "Nonperturbative partonic quasidistributions of the pion from chiral quark models," Phys. Lett. B 773, 385-390, arXiv:1707.09588 [hep-ph]

Broniowski, Wojciech, and Enrique Ruiz Arriola (2018), "Partonic quasidistributions of the proton and pion from transverse-momentum distributions," Phys. Rev. D 97 (3), 034031, arXiv:1711.03377 [hep-ph],

Bunce, Gerry, Naohito Saito, Jacques Soffer, and Werner Vogelsang (2000), "Prospects for spin physics at RHIC," Ann. Rev. Nucl. Part. Sci. 50, 525-575, arXiv:hep-ph/0007218 [hep-ph].

Burkardt, M (1989), "The Virial Theorem and the Structure of the Deuteron in (1+1)-dimensional QCD on the Light Cone," Nucl. Phys. A504, 762-776

Burkardt, Matthias (1993), "Light front quantization of the Sine-Gordon model," Phys. Rev. D47, 4628-4633.

Burkardt, Matthias (2000), "Impact parameter dependent parton distributions and off forward parton distributions for zeta $\longrightarrow$ 0," Phys. Rev. D62, 071503, [Erratum: Phys. Rev.D66,119903(2002)], arXiv:hep-ph/0005108 [hep-ph].

Burkhardt, Hugh, and W.N. Cottingham (1970), "Sum rules for forward virtual Compton scattering," Annals Phys. 56, 453-463.

Cammarota, Justin, Leonard Gamberg, Zhong-Bo Kang, Joshua A. Miller, Daniel Pitonyak, Alexei Prokudin, Ted C. Rogers, and Nobuo Sato (2020), "The origin of single transverse-spin asymmetries in high-energy collisions," arXiv:2002.08384 [hep-ph].

Capitani, S, M. Gockeler, R. Horsley, H. Oelrich, D. Petters, Paul E.L. Rakow, and G. Schierholz (1999a), "Towards a nonperturbative calculation of DIS Wilson coefficients," Nucl. Phys. B Proc. Suppl. 73, 288-290, arXiv:hep-lat/9809171

Capitani, S, M. Gockeler, R. Horsley, D. Petters, D. Pleiter, Paul E.L. Rakow, and G. Schierholz (1999b), "Higher twist corrections to nucleon structure functions from lattice QCD," Nucl. Phys. B Proc. Suppl. 79, 173-175. arXiv:hep-ph/9906320.

Carlson, Carl E, and Michael Freid (2017), "Lattice corrections to the quark quasidistribution at one-loop," Phys. Rev. D 95 (9), 094504, arXiv:1702.05775 [hep-ph],

Catani, S, and M. Grazzini (2012), "Higgs Boson Production at Hadron Colliders: Hard-Collinear Coefficients at the NNLO," Eur. Phys. J. C72, 2013, [Erratum: Eur. Phys. J.C72,2132(2012)], arXiv:1106.4652 [hep-ph]

Catani, Stefano, Leandro Cieri, Daniel de Florian, Giancarlo Ferrera, and Massimiliano Grazzini (2012), "Vector boson production at hadron colliders: hard-collinear coefficients at the NNLO," Eur. Phys. J. C72, 2195, arXiv:1209.0158 [hep-ph].

Chadwick, J (1932), "Possible Existence of a Neutron," Nature 129, 312

Chai, Yahui, et al. (2020), "Parton distribution functions of $\Delta^{+}$on the lattice," arXiv:2002.12044 [hep-lat].

Chambers, AJ, R. Horsley, Y. Nakamura, H. Perlt, P.E.L. Rakow, G. Schierholz, A. Schiller, K. Somfleth, R.D. Young, and J.M. Zanotti (2017), "Nucleon Structure Functions from Operator Product Expansion on the Lattice," Phys. Rev. Lett. 118 (24), 242001. arXiv:1703.01153 [hep-lat]. 
Chang, C C, et al. (2018), "A per-cent-level determination of the nucleon axial coupling from quantum chromodynamics," Nature 558 (7708), 91-94, arXiv:1805.12130 [hep-lat]

Chang, Lei, I. C. Cloet, J. J. Cobos-Martinez, C. D. Roberts, S. M. Schmidt, and P. C. Tandy (2013), "Imaging dynamical chiral symmetry breaking: pion wave function on the light front," Phys. Rev. Lett. 110 (13), 132001. arXiv:1301.0324 [nucl-th]

Chang, Shau-Jin, and Shang-Keng Ma (1969), "Feynman rules and quantum electrodynamics at infinite momentum," Phys. Rev. 180, 1506-1513

Chang, Wen-Chen, and Jen-Chieh Peng (2014), "Flavor Structure of the Nucleon Sea," $\quad$ Prog. Part. Nucl. Phys. 79, 95-135. arXiv:1406.1260 [hep-ph]

Chen, Jiunn-Wei, Saul D. Cohen, Xiangdong Ji, HueyWen Lin, and Jian-Hui Zhang (2016), "Nucleon Helicity and Transversity Parton Distributions from Lattice QCD," Nucl. Phys. B911, 246-273, arXiv:1603.06664 [hep-ph].

Chen, Jiunn-Wei, Tomomi Ishikawa, Luchang Jin, HueyWen Lin, Yi-Bo Yang, Jian-Hui Zhang, and Yong Zhao (2018), "Parton distribution function with nonperturbative renormalization from lattice QCD," Phys. Rev. D97 (1), 014505, arXiv:1706.01295 [hep-lat],

Chen, Jiunn-Wei, Tomomi Ishikawa, Luchang Jin, HueyWen Lin, Jian-Hui Zhang, and Yong Zhao (LP3) (2019a), "Symmetry properties of nonlocal quark bilinear operators on a Lattice," Chin. Phys. C43 (10), 103101. arXiv:1710.01089 [hep-lat]

Chen, Jiunn-Wei, Xiangdong Ji, and Jian-Hui Zhang (2017), "Improved quasi parton distribution through Wilson line renormalization," Nucl. Phys. B915, 1-9, arXiv:1609.08102 [hep-ph].

Chen, Jiunn-Wei, Huey-Wen Lin, and Jian-Hui Zhang (2019b), "Pion generalized parton distribution from lattice QCD," $10.1016 /$ j.nuclphysb.2020.114940 arXiv:1904.12376 [hep-lat]

Chen, Long-Bin, Wei Wang, and Ruilin Zhu (2020a), "Master integrals for two-loop QCD corrections to quark quasi PDFs," JHEP 10, 079, arXiv:2006.10917 [hep-ph].

Chen, Long-Bin, Wei Wang, and Ruilin Zhu (2020b), "Nextto-next-to-leading order corrections to quark Quasi parton distribution functions," arXiv:2006.14825 [hep-ph]

Chen, Long-Bin, Wei Wang, and Ruilin Zhu (2020c), "Quasi parton distribution functions at NNLO: flavor non-diagonal quark contributions," Phys. Rev. D 102 (1), 011503, arXiv:2005.13757 [hep-ph]

Chen, Xiang-Song, Wei-Min Sun, Fan Wang, and T. Goldman (2011), "Proper identification of the gluon spin," Phys. Lett. B700, 21-24, arXiv:1101.5358 [hep-ph]

Chernyak, V L, and A. R. Zhitnitsky (1982), "Exclusive Decays of Heavy Mesons," Nucl. Phys. B201, 492, [Erratum: Nucl. Phys.B214,547(1983)].

Chetyrkin, K G, and A. G. Grozin (2003), "Three loop anomalous dimension of the heavy light quark current in HQET," Nucl. Phys. B666, 289-302, arXiv:hep-ph/0303113 [hep-ph]

Chiu, Jui-Yu, Ambar Jain, Duff Neill, and Ira Z. Rothstein (2012), "A Formalism for the Systematic Treatment of Rapidity Logarithms in Quantum Field Theory," JHEP 05, 084, arXiv:1202.0814 [hep-ph].

Christ, Norman H, B. Hasslacher, and Alfred H. Mueller (1972), "Light cone behavior of perturbation theory,"
Phys. Rev. D 6, 3543 .

Cichy, Krzysztof, and Martha Constantinou (2019), "A guide to light-cone PDFs from Lattice QCD: an overview of approaches, techniques and results," Adv. High Energy Phys. 2019, 3036904, arXiv:1811.07248 [hep-lat].

Cichy, Krzysztof, Luigi Del Debbio, and Tommaso Giani (2019), "Parton distributions from lattice data: the nonsinglet case," JHEP 10, 137, arXiv:1907.06037 [hep-ph].

Collins, J C, A. V. Efremov, K. Goeke, M. Grosse Perdekamp, S. Menzel, B. Meredith, A. Metz, and P. Schweitzer (2005), "Sivers effect in semi-inclusive deeply inelastic scattering and Drell-Yan," in Transversity. Proceedings, Workshop, Como, Italy, September 7-10, 20 pp. 212-219, arXiv:hep-ph/0510342 [hep-ph]

Collins, J C, A. V. Efremov, K. Goeke, S. Menzel, A. Metz, and P. Schweitzer (2006), "Sivers effect in semi-inclusive deeply inelastic scattering," Phys. Rev. D73, 014021, arXiv:hep-ph/0509076 [hep-ph].

Collins, John (2008), "Rapidity divergences and valid definitions of parton densities," Proceedings, International Workshop on Relativistic nuclear and particle physics (Light Cone 2008): Mulhouse, France, July 7-11, 2008, PoS LC2008, 028, arXiv:0808.2665 [hep-ph]

Collins, John (2011a), "Foundations of perturbative QCD," Camb. Monogr. Part. Phys. Nucl. Phys. Cosmol. 32, 1624.

Collins, John (2011b), "New definition of TMD parton densities," Proceedings, QCD Evolution Workshop on From Collinear to Non-Collinear Case: Newport News, Virginia, April 8-9, 2011, Int. J. Mod. Phys. Conf. Ser. 4, 85-96. arXiv:1107.4123 [hep-ph]

Collins, John, and Ted C. Rogers (2017), "Connecting Different TMD Factorization Formalisms in QCD," Phys. Rev. D96 (5), 054011, arXiv:1705.07167 [hep-ph]

Collins, John C (1986), Renormalization, Cambridge Monographs on Mathematical Physics, Vol. 26 (Cambridge University Press, Cambridge).

Collins, John C (1993), "Fragmentation of transversely polarized quarks probed in transverse momentum distributions," Nucl. Phys. B396, 161-182, arXiv:hep-ph/9208213 [hep-ph].

Collins, John C (2002), "Leading twist single transversespin asymmetries: Drell-Yan and deep inelastic scattering," Phys. Lett. B536, 43-48, arXiv:hep-ph/0204004 [hep-ph].

Collins, John C, and Andreas Metz (2004), "Universality of soft and collinear factors in hardscattering factorization," Phys. Rev. Lett. 93, 252001. arXiv:hep-ph/0408249 [hep-ph].

Collins, John C, and Ted C. Rogers (2013), "Equality of Two Definitions for Transverse Momentum Dependent Parton Distribution Functions," Phys. Rev. D87 (3), 034018, arXiv:1210.2100 [hep-ph]

Collins, John C, and Randall J. Scalise (1994), "The Renormalization of composite operators in Yang-Mills theories using general covariant gauge," Phys. Rev. D50, 4117-4136. arXiv:hep-ph/9403231 [hep-ph].

Collins, John C, and Davison E. Soper (1981), "Back-To-Back Jets in QCD," Nucl. Phys. B193, 381, [Erratum: Nucl. Phys.B213,545(1983)].

Collins, John C, and Davison E. Soper (1982a), "Back-ToBack Jets: Fourier Transform from B to K-Transverse," Nucl. Phys. B197, 446-476 
Collins, John C, and Davison E. Soper (1982b), "Parton Distribution and Decay Functions," Nucl. Phys. B194, 445-492

Collins, John C, Davison E. Soper, and George F. Sterman (1983), "Factorization for One Loop Corrections in the Drell-Yan Process," Nucl. Phys. B223, 381-421

Collins, John C, Davison E. Soper, and George F. Sterman (1985a), "Factorization for Short Distance Hadron - Hadron Scattering," Nucl. Phys. B261, 104-142.

Collins, John C, Davison E. Soper, and George F. Sterman (1985b), "Transverse Momentum Distribution in Drell-Yan Pair and W and Z Boson Production," Nucl. Phys. B250, 199-224.

Collins, John C, Davison E. Soper, and George F. Sterman (1988), "Soft Gluons and Factorization," Nucl. Phys. B308, 833-856

Constantinou, Martha (2020), "The x-dependence of hadronic parton distributions: A review on the progress of lattice QCD," in 38th International Symposium on Lattice Field Theory, arXiv:2010.02445 [hep-lat]

Constantinou, Martha, and Haralambos Panagopoulos (2017), "Perturbative renormalization of quasi-parton distribution functions," Phys. Rev. D96 (5), 054506 arXiv:1705.11193 [hep-lat]

Constantinou, Martha, Haralambos Panagopoulos, and Gregoris Spanoudes (2019), "One-loop renormalization of staple-shaped operators in continuum and lattice regularizations," Phys. Rev. D99 (7), 074508, arXiv:1901.03862 [hep-lat]

Constantinou, Martha, et al. (2020), "Parton distributions and lattice QCD calculations: toward 3D structure," arXiv:2006.08636 [hep-ph]

Courtoy, Aurore, Gary R. Goldstein, J. Osvaldo Gonzalez Hernandez, Simonetta Liuti, and Abha Rajan (2014), "On the Observability of the Quark Orbital Angular Momentum Distribution," Phys. Lett. B731, 141-147, arXiv:1310.5157 [hep-ph]

Craigie, N S, and Harald Dorn (1981), "On the Renormalization and Short Distance Properties of Hadronic Operators in QCD," Nucl. Phys. B185, 204-220.

Curci, G, W. Furmanski, and R. Petronzio (1980), "Evolution of Parton Densities Beyond Leading Order: The Nonsinglet Case," Nucl. Phys. B175, 27-92.

D'Alesio, Umberto, Miguel G. Echevarria, Stefano Melis, and Ignazio Scimemi (2014), "Non-perturbative QCD effects in $q_{T}$ spectra of Drell-Yan and Z-boson production," JHEP 11, 098, arXiv:1407.3311 [hep-ph].

Dasgupta, M, and B. R. Webber (1996), "Power corrections and renormalons in deep inelastic structure functions," $\quad$ Phys. Lett. B382, 273-281, arXiv:hep-ph/9604388 [hep-ph]

Dasgupta, M, and B. R. Webber (1997), "Power corrections and renormalons in $e^{+} e^{-}$fragmentation functions," Nucl. Phys. B484, 247-264, arXiv:hep-ph/9608394 [hep-ph].

Dawson, C, G. Martinelli, G.C. Rossi, Christopher T. Sachrajda, Stephen R. Sharpe, M. Talevi, and M. Testa (1998), "New lattice approaches to the delta I $=1 / 2$ rule," Nucl. Phys. B 514, 313-335, arXiv:hep-lat/9707009

Deka, M, T. Streuer, T. Doi, S. J. Dong, T. Draper, K. F. Liu, N. Mathur, and A. W. Thomas (2009), "Moments of Nucleon's Parton Distribution for the Sea and Valence Quarks from Lattice QCD," Phys. Rev. D79, 094502 . arXiv:0811.1779 [hep-ph]
Del Debbio, Luigi, Tommaso Giani, Joseph Karpie, Kostas Orginos, Anatoly Radyushkin, and Savvas Zafeiropoulos (2020a), "Neural-network analysis of Parton Distribution Functions from Ioffe-time pseudodistributions," arXiv:2010.03996 [hep-ph]

Del Debbio, Luigi, Tommaso Giani, and Christopher J. Monahan (2020b), "Notes on lattice observables for parton distributions: nongauge theories," JHEP 09, 021, arXiv:2007.02131 [hep-lat].

Dennison, David M (1927), "A Note on the Specific Heat of the Hydrogen Molecule," Proceedings of the Royal Society of London 115, 483-486.

Detmold, William, Anthony V. Grebe, Issaku Kanamori, C.J. David Lin, Santanu Mondal, Robert J. Perry, and Yong Zhao (2020), "A Preliminary Determination of the Second Mellin Moment of the Pion's Distribution Amplitude Using the Heavy Quark Operator Product Expansion," in Asia-Pacific Symposium for Lattice Field Theory, arXiv:2009.09473 [hep-lat]

Detmold, William, Issaku Kanamori, C.J. David Lin, Santanu Mondal, and Yong Zhao (2018), "Moments of pion distribution amplitude using operator product expansion on the lattice," PoS LATTICE2018, 106 , arXiv:1810.12194 [hep-lat]

Detmold, William, and C. J. David Lin (2006), "Deep-inelastic scattering and the operator product expansion in lattice QCD," Phys. Rev. D73, 014501, arXiv:hep-lat/0507007 [hep-lat]

Deur, Alexandre, Stanley J. Brodsky, and Guy F. De Téramond (2019), "The Spin Structure of the Nucleon," Rept. Prog. Phys. 82 (076201), 10.1088/1361-6633/ab0b8f arXiv:1807.05250 [hep-ph].

Diehl, M (2003), "Generalized parton distributions," Phys. Rept. 388, 41-277, arXiv:hep-ph/0307382 [hep-ph].

Dirac, Paul A M (1949), "Forms of Relativistic Dynamics," Rev. Mod. Phys. 21, 392-399.

Dokshitzer, Yuri L (1977), "Calculation of the Structure Functions for Deep Inelastic Scattering and e+ e- Annihilation by Perturbation Theory in Quantum Chromodynamics." Sov. Phys. JETP 46, 641-653, [Zh. Eksp. Teor. Fiz.73,1216(1977)].

Dokshitzer, Yuri L, G. Marchesini, and B. R. Webber (1996), "Dispersive approach to power behaved contributions in QCD hard processes," Nucl. Phys. B469, 93-142, arXiv:hep-ph/9512336 [hep-ph]

Dolgov, D, et al. (LHPC, TXL) (2002), "Moments of nucleon light cone quark distributions calculated in full lattice QCD," Phys. Rev. D66, 034506, arXiv:hep-lat/0201021 [hep-lat]

Dorn, Harald (1986), "Renormalization of Path Ordered Phase Factors and Related Hadron Operators in Gauge Field Theories," Fortsch. Phys. 34, 11-56

Dotsenko, V S, and S. N. Vergeles (1980), "Renormalizability of Phase Factors in the Nonabelian Gauge Theory," Nucl. Phys. B169, 527-546

Drell, S D, and Tung-Mow Yan (1971), "Partons and their Applications at High-Energies," Annals Phys. 66, 578, [Annals Phys.281,450(2000)].

Ebert, Markus A, Stella T. Schindler, Iain W. Stewart, and Yong Zhao (2020a), "One-loop Matching for Spin-Dependent Quasi-TMDs," JHEP 09, 099, arXiv:2004.14831 [hep-ph]

Ebert, Markus A, Iain W. Stewart, and Yong Zhao (2019a), "Determining the Nonperturbative Collins-Soper 
Kernel From Lattice QCD," Phys. Rev. D99 (3), 034505 arXiv:1811.00026 [hep-ph]

Ebert, Markus A, Iain W. Stewart, and Yong Zhao (2019b), "Towards Quasi-Transverse Momentum Dependent PDFs Computable on the Lattice," JHEP 09, 037. arXiv:1901.03685 [hep-ph].

Ebert, Markus A, Iain W. Stewart, and Yong Zhao (2020b), "Renormalization and Matching for the Collins-Soper Kernel from Lattice QCD," JHEP 03, 099 arXiv:1910.08569 [hep-ph]

Echevarría, Miguel G, Ahmad Idilbi, and Ignazio Scimemi (2013), "Soft and Collinear Factorization and Transverse Momentum Dependent Parton Distribution Functions," Phys. Lett. B726, 795-801, arXiv:1211.1947 [hep-ph]

Echevarria, Miguel G, Ahmad Idilbi, Zhong-Bo Kang, and Ivan Vitev (2014), "QCD Evolution of the Sivers Asymmetry," Phys. Rev. D89, 074013, arXiv:1401.5078 [hep-ph]

Echevarria, Miguel G, Ahmad Idilbi, and Ignazio Scimemi (2012), "Factorization Theorem For Drell-Yan At Low $q_{T}$ And Transverse Momentum Distributions On-The-LightCone," JHEP 07, 002, arXiv:1111.4996 [hep-ph].

Echevarria, Miguel G, Ignazio Scimemi, and Alexey Vladimirov (2016a), "Transverse momentum dependent fragmentation function at next-to-next-to-leading order," Phys. Rev. D93 (1), 011502, [Erratum: Phys. Rev.D94,no.9,099904(2016)], arXiv:1509.06392 [hep-ph].

Echevarria, Miguel G, Ignazio Scimemi, and Alexey Vladimirov (2016b), "Universal transverse momentum dependent soft function at NNLO," Phys. Rev. D93 (5), 054004, arXiv:1511.05590 [hep-ph]

Echevarria, Miguel G, Ignazio Scimemi, and Alexey Vladimirov (2016c), "Unpolarized Transverse Momentum Dependent Parton Distribution and Fragmentation Functions at next-to-next-to-leading order," JHEP 09, 004, arXiv:1604.07869 [hep-ph]

Efremov, A V, K. Goeke, S. Menzel, A. Metz, and P. Schweitzer (2005), "Sivers effect in semi-inclusive DIS and in the Drell-Yan process," Phys. Lett. B612, 233-244, arXiv:hep-ph/0412353 [hep-ph]

Efremov, AV, and A.V. Radyushkin (1980), "Asymptotical Behavior of Pion Electromagnetic Form-Factor in QCD," Theor. Math. Phys. 42, 97-110

Egerer, Colin, Robert G. Edwards, Kostas Orginos, and David G. Richards (2020), "Distillation at HighMomentum," arXiv:2009.10691 [hep-lat].

Ellis, R Keith, W. Furmanski, and R. Petronzio (1983), "Unraveling Higher Twists," Nucl. Phys. B212, 29.

Engelhardt, M (2017), "Quark orbital dynamics in the proton from Lattice QCD - from Ji to Jaffe-Manohar orbital angular momentum," Phys. Rev. D95 (9), 094505. arXiv:1701.01536 [hep-lat]

Engelhardt, M, J. Green, N. Hasan, S. Krieg, S. Meinel, J. Negele, A. Pochinsky, and S. Syritsyn (2018), "Quark orbital angular momentum in the proton evaluated using a direct derivative method," Proceedings, 36th International Symposium on Lattice Field Theory (Lattice 2018): East Lansing, MI, United States, July 22-28, 2018, PoS LATTICE2018, 115, arXiv:1901.00843 [hep-lat].

Engelhardt, M, P. Hägler, B. Musch, J. Negele, and A. Schäfer (2016), "Lattice QCD study of the BoerMulders effect in a pion," Phys. Rev. D93 (5), 054501. arXiv:1506.07826 [hep-lat]

Estermann, I, R. FRISCH, and O. Stern (1933), "Magnetic Moment of the Proton," Nature 132, 169-170
Ethier, J J, N. Sato, and W. Melnitchouk (2017), "First simultaneous extraction of spin-dependent parton distributions and fragmentation functions from a global QCD analysis," Phys. Rev. Lett. 119 (13), 132001 arXiv:1705.05889 [hep-ph].

Fan, Zhou-You, Yi-Bo Yang, Adam Anthony, HueyWen Lin, and Keh-Fei Liu (2018), "Gluon Quasi-Parton-Distribution Functions from Lattice QCD," Phys. Rev. Lett. 121 (24), 242001 arXiv:1808.02077 [hep-lat]

Fan, Zhouyou, Xiang Gao, Ruizi Li, Huey-Wen Lin, Nikhil Karthik, Swagato Mukherjee, Peter Petreczky, Sergey Syritsyn, Yi-Bo Yang, and Rui Zhang (2020a), "Isovector parton distribution functions of the proton on a superfine lattice," Phys. Rev. D 102 (7), 074504 arXiv:2005.12015 [hep-lat]

Fan, Zhouyou, Rui Zhang, and Huey-Wen Lin (2020b), "Nucleon Gluon Distribution Function from $2+1+1$-Flavor Lattice QCD," arXiv:2007.16113 [hep-lat].

Farrar, Glennys R, and Darrell R. Jackson (1979), "The Pion Form-Factor," Phys. Rev. Lett. 43, 246

Favart, L, M. Guidal, T. Horn, and P. Kroll (2016), "Deeply Virtual Meson Production on the nucleon," Eur. Phys. J. A52 (6), 158, arXiv:1511.04535 [hep-ph]

Feynman, R P (1972), Photon-hadron interactions (CRC Press).

Feynman, Richard P (1969), "Very high-energy collisions of hadrons," Phys. Rev. Lett. 23, 1415-1417.

Filippone, B W, and Xiang-Dong Ji (2001), "The Spin structure of the nucleon," Adv. Nucl. Phys. 26, 1 , arXiv:hep-ph/0101224 [hep-ph],

de Florian, Daniel, Rodolfo Sassot, Marco Stratmann, and Werner Vogelsang (2014), "Evidence for polarization of gluons in the proton," Phys. Rev. Lett. 113 (1), 012001, arXiv:1404.4293 [hep-ph]

Fritzsch, H, Murray Gell-Mann, and H. Leutwyler (1973), "Advantages of the Color Octet Gluon Picture," Phys. Lett. B 47, 365-368

Gamberg, Leonard, Zhong-Bo Kang, Ivan Vitev, and Hongxi Xing (2015), "Quasi-parton distribution functions: a study in the diquark spectator model," Phys. Lett. B743, 112-120, arXiv:1412.3401 [hep-ph]

Gao, Jun, Lucian Harland-Lang, and Juan Rojo (2018), "The Structure of the Proton in the LHC Precision Era," Phys. Rept. 742, 1-121, arXiv:1709.04922 [hep-ph].

Gao, Xiang, Luchang Jin, Christos Kallidonis, Nikhil Karthik, Swagato Mukherjee, Peter Petreczky, Charles Shugert, Sergey Syritsyn, and Yong Zhao (2020), "Valence parton distribution of pion from lattice QCD: Approaching continuum," arXiv:2007.06590 [hep-lat]

Geesaman, D F, and P. E. Reimer (2019), "The sea of quarks and antiquarks in the nucleon," $\quad$ Rept. Prog. Phys. 82 (4), 046301. arXiv:1812.10372 [nucl-ex]

Gehrmann, T, E.W.N. Glover, T. Huber, N. Ikizlerli, and C. Studerus (2010), "Calculation of the quark and gluon form factors to three loops in QCD," JHEP 06, 094, arXiv:1004.3653 [hep-ph].

Gehrmann, Thomas, Thomas Luebbert, and Li Lin Yang (2014), "Calculation of the transverse parton distribution functions at next-to-next-to-leading order," JHEP 06, 155 , arXiv:1403.6451 [hep-ph].

Gervais, Jean-Loup, and A. Neveu (1980), "The Slope of the Leading Regge Trajectory in Quantum Chromodynamics," 
Nucl. Phys. B 163, 189-216.

Gockeler, M, R. Horsley, D. Pleiter, Paul E. L. Rakow, A. Schafer, G. Schierholz, and W. Schroers (QCDSF) (2004), "Generalized parton distributions from lattice QCD," Phys. Rev. Lett. 92, 042002 arXiv:hep-ph/0304249 [hep-ph]

Gong, Ming, Yi-Bo Yang, Jian Liang, Andrei Alexandru, Terrence Draper, and Keh-Fei Liu ( $\chi$ QCD) (2017), "Strange and charm quark spins from the anomalous Ward identity," Phys. Rev. D95 (11), 114509, arXiv:1511.03671 [hep-ph]

Green, J R, M. Engelhardt, S. Krieg, J. W. Negele, A. V. Pochinsky, and S. N. Syritsyn (2014), "Nucleon Structure from Lattice QCD Using a Nearly Physical Pion Mass," Phys. Lett. B734, 290-295, arXiv:1209.1687 [hep-lat].

Green, Jeremy, Karl Jansen, and Fernanda Steffens (2018), "Nonperturbative Renormalization of Nonlocal Quark Bilinears for Parton Quasidistribution Functions on the Lattice Using an Auxiliary Field," Phys. Rev. Lett. 121 (2), 022004 arXiv:1707.07152 [hep-lat]

Green, Jeremy R, Karl Jansen, and Fernanda Steffens (2020), "Improvement, generalization, and scheme conversion of Wilson-line operators on the lattice in the auxiliary field approach," arXiv:2002.09408 [hep-lat]

Gribov, V N, and L. N. Lipatov (1972), "Deep inelastic e p scattering in perturbation theory," Sov. J. Nucl. Phys. 15, 438-450, [Yad. Fiz.15,781(1972)].

Gross, David J, and Frank Wilczek (1973), "Ultraviolet Behavior of Nonabelian Gauge Theories," Phys. Rev. Lett. 30, 1343-1346.

Grozin, Andrey, Johannes M. Henn, Gregory P. Korchemsky, and Peter Marquard (2016), "The three-loop cusp anomalous dimension in QCD and its supersymmetric extensions," JHEP 01, 140, arXiv:1510.07803 [hep-ph]

Grozin, Andrey G (2005), "B-meson distribution amplitudes," Helmholtz International Summer School on Heavy Quark Physics Moscow, Dubna, Russia, June 6-16, 2005, Int. J. Mod. Phys. A20, 7451-7484, arXiv:hep-ph/0506226 [hep-ph].

Gupta, Rajan, David Daniel, and Jeffrey Grandy (1993), "Bethe-Salpeter amplitudes and density correlations for mesons with Wilson fermions," Phys. Rev. D48, 3330-3339, arXiv:hep-lat/9304009 [hep-lat].

Hagler, Ph (2010), "Hadron structure from lattice quantum chromodynamics," Phys. Rept. 490, 49-175 arXiv:0912.5483 [hep-lat].

Hagler, Ph, B. U. Musch, J. W. Negele, and A. Schafer (2009), "Intrinsic quark transverse momentum in the nucleon from lattice QCD," EPL 88 (6), 61001 arXiv:0908.1283 [hep-lat].

Hagler, Ph, et al. (LHPC) (2008), "Nucleon Generalized Parton Distributions from Full Lattice QCD," Phys. Rev. D77, 094502, arXiv:0705.4295 [hep-lat]

Hannaford-Gunn, A, R. Horsley, Y. Nakamura, H. Perlt, P.E.L. Rakow, G. Schierholz, K. Somfleth, H. Stüben, R.D. Young, and J.M. Zanotti (2020), "Scaling and higher twist in the nucleon Compton amplitude," PoS LATTICE2019, 278, arXiv:2001.05090 [hep-lat].

Harada, Koji, Atsushi Okazaki, and Masa-aki Taniguchi (1996), "Mesons in the massive Schwinger model on the light cone," Phys. Rev. D54, 7656-7663. arXiv:hep-th/9509136 [hep-th]

Harindranath, A, and J. P. Vary (1987), "SOLVING
TWO-DIMENSIONAL PHI**4 THEORY BY DISCRETIZED LIGHT FRONT QUANTIZATION," Phys. Rev. D36, 1141-1147.

Harland-Lang, L A, A. D. Martin, P. Motylinski, and R. S. Thorne (2015), "Parton distributions in the LHC era: MMHT 2014 PDFs," Eur. Phys. J. C75 (5), 204, arXiv:1412.3989 [hep-ph]

Hashimoto, Shoji, and Hideo Matsufuru (1996), "Lattice heavy quark effective theory and the Isgur-Wise function," Phys. Rev. D54, 4578-4584 arXiv:hep-lat/9511027 [hep-lat]

Hatta, Yoshitaka (2012), "Notes on the orbital angular momentum of quarks in the nucleon," Phys. Lett. B708, 186-190, arXiv:1111.3547 [hep-ph]

Hatta, Yoshitaka, Xiangdong Ji, and Yong Zhao (2014), "Gluon helicity $\Delta G$ from a universality class of operators on a lattice," Phys. Rev. D89 (8), 085030. arXiv:1310.4263 [hep-ph].

Hatta, Yoshitaka, Yuya Nakagawa, Feng Yuan, Yong Zhao, and Bowen Xiao (2017), "Gluon orbital angular momentum at small- $x$," Phys. Rev. D95 (11), 114032 arXiv:1612.02445 [hep-ph]

Hatta, Yoshitaka, and Shinsuke Yoshida (2012), "Twist analysis of the nucleon spin in QCD," JHEP 10,080, arXiv:1207.5332 [hep-ph].

Heinzl, Th, St. Krusche, and E. Warner (1991), "Nontrivial vacuum structure in light-cone quantum field theory," Nucl. Phys. A532, 429-434.

Henn, Johannes M, Gregory P. Korchemsky, and Bernhard Mistlberger (2019), "The full four-loop cusp anomalous dimension in $\mathcal{N}=4$ super Yang-Mills and QCD," arXiv:1911.10174 [hep-th]

Hobbs, T J, Bo-Ting Wang, Pavel M. Nadolsky, and Fredrick I. Olness (2019), "Charting the coming synergy between lattice QCD and high-energy phenomenology," Phys. Rev. D100 (9), 094040, arXiv:1904.00022 [hep-ph]

Hobbs, TJ (2018), "Quantifying finite-momentum effects in the quark quasidistribution functions of mesons," Phys. Rev. D 97 (5), 054028, arXiv:1708.05463 [hep-ph].

Hofstadter, Robert (1956), "Electron scattering and nuclear structure," Rev. Mod. Phys. 28, 214-254

Holt, Roy J, and Craig D. Roberts (2010), "Distribution Functions of the Nucleon and Pion in the Valence Region," Rev. Mod. Phys. 82, 2991-3044, arXiv:1002.4666 [nucl-th].

Hoodbhoy, Pervez, Xiang-Dong Ji, and Wei Lu (1999a), "Implications of color gauge symmetry for nucleon spin structure," Phys. Rev. D59, 074010, arXiv:hep-ph/9808305 [hep-ph].

Hoodbhoy, Pervez, Xiang-Dong Ji, and Wei Lu (1999b), "Quark orbital - angular - momentum distribution in the nucleon," Phys. Rev. D 59, 014013 , arXiv:hep-ph/9804337.

't Hooft, Gerard (1974), "A Two-Dimensional Model for Mesons," Nucl. Phys. B75, 461-470.

Horgan, R R, et al. (2009), "Moving NRQCD for heavy-tolight form factors on the lattice," Phys. Rev. D80, 074505 . arXiv:0906.0945 [hep-lat]

Horsley, Roger, Yoshifumi Nakamura, Holger Perlt, Paul E.L. Rakow, Gerrit Schierholz, Kim Somfleth, Ross D. Young, and James M. Zanotti (QCDSF-UKQCD-CSSM) (2020), "Structure functions from the Compton amplitude," PoS LATTICE2019, 137, arXiv:2001.05366 [hep-lat] Hou, Tie-Jiun, et al. (2019), "New CTEQ global analysis of 
quantum chromodynamics with high-precision data from the LHC," arXiv:1912.10053 [hep-ph].

Ishikawa, Tomomi, Yan-Qing Ma, Jian-Wei Qiu, and Shinsuke Yoshida (2016), "Practical quasi parton distribution functions," arXiv:1609.02018 [hep-lat]

Ishikawa, Tomomi, Yan-Qing Ma, Jian-Wei Qiu, and Shinsuke Yoshida (2017), "Renormalizability of quasiparton distribution functions," Phys. Rev. D96 (9), 094019. arXiv:1707.03107 [hep-ph]

Izubuchi, Taku, Xiangdong Ji, Luchang Jin, Iain W. Stewart, and Yong Zhao (2018), "Factorization Theorem Relating Euclidean and Light-Cone Parton Distributions," Phys. Rev. D98 (5), 056004, arXiv:1801.03917 [hep-ph],

Izubuchi, Taku, Luchang Jin, Christos Kallidonis, Nikhil Karthik, Swagato Mukherjee, Peter Petreczky, Charles Shugert, and Sergey Syritsyn (2019), "Valence parton distribution function of pion from fine lattice," Phys. Rev. D100 (3), 034516, arXiv:1905.06349 [hep-lat]

Jaffe, R L, and Xiang-Dong Ji (1991), "Chiral odd parton distributions and polarized Drell-Yan," Phys. Rev. Lett. 67, 552-555.

Jaffe, R L, and Xiang-Dong Ji (1992), "Chiral odd parton distributions and Drell-Yan processes," Nucl. Phys. B375, 527-560

Jaffe, R L, and Aneesh Manohar (1990), "The G(1) Problem: Fact and Fantasy on the Spin of the Proton," Nucl. Phys. B337, 509-546.

Jaffe, R L, and M. Soldate (1982), "Twist Four in Electroproduction: Canonical Operators and Coefficient Functions," Phys. Rev. D26, 49-68.

$\mathrm{Ji}$, X (2004), "Generalized parton distributions," Ann. Rev. Nucl. Part. Sci. 54, 413-450.

Ji, Xiang-Dong (1992), "Gluon correlations in the transversely polarized nucleon," Phys. Lett. B289, 137-142

Ji, Xiang-Dong (1993), "The Nucleon structure functions from deep inelastic scattering with electroweak currents," Nucl. Phys. B402, 217-250

$\mathrm{Ji}$, Xiang-Dong (1995), "Infrared renormalons and power corrections in deep inelastic sum rules," Nucl. Phys. B448, 51-66, arXiv:hep-ph/9411312 [hep-ph]

Ji, Xiang-Dong (1997a), "Deeply virtual Compton scattering," Phys. Rev. D55, 7114-7125 arXiv:hep-ph/9609381 [hep-ph]

Ji, Xiang-Dong (1997b), "Gauge-Invariant Decomposition of Nucleon Spin," Phys. Rev. Lett. 78, 610-613. arXiv:hep-ph/9603249 [hep-ph]

$\mathrm{Ji}$, Xiang-Dong (1998), "Off forward parton distributions," J. Phys. G24, 1181-1205, arXiv:hep-ph/9807358 [hep-ph]

Ji, Xiang-dong (2003), "Viewing the proton through 'color' filters," $\quad$ Phys. Rev. Lett. 91, 062001. arXiv:hep-ph/0304037 [hep-ph]

Ji, Xiang-Dong, and Chi-hong Chou (1990), "QCD radiative corrections to the transverse spin structure function g2 (x, $\left.\mathrm{Q}^{* *} 2\right)$ : 1. Nonsinglet operators," Phys. Rev. D42, 3637-3644.

Ji, Xiang-dong, and Chul-woo Jung (2001), "Studying hadronic structure of the photon in lattice QCD," Phys. Rev. Lett. 86, 208, arXiv:hep-lat/0101014.

Ji, Xiang-dong, Jian-Ping Ma, and Feng Yuan (2004), "QCD factorization for spin-dependent cross sections in DIS and Drell-Yan processes at low transverse momentum," Phys. Lett. B597, 299-308. arXiv:hep-ph/0405085 [hep-ph]

Ji, Xiang-dong, Jian-ping Ma, and Feng Yuan (2005), "QCD factorization for semi-inclusive deep-inelastic scattering at low transverse momentum," Phys. Rev. D71, 034005 arXiv:hep-ph/0404183 [hep-ph]

Ji, Xiang-Dong, and M. J. Musolf (1991), "Subleading logarithmic mass dependence in heavy meson form-factors," Phys. Lett. B257, 409-413

Ji, Xiang-Dong, and Jonathan Osborne (2001), "An Analysis of the next-to-leading order corrections to the $\mathrm{g}(\mathrm{T})=$ $\mathrm{g}(1)+\mathrm{g}(\mathrm{s}))$ scaling function," Nucl. Phys. B608, 235-278. arXiv:hep-ph/0102026 [hep-ph].

$\mathrm{Ji}$, Xiangdong (2013), "Parton Physics on a Euclidean Lattice," $\quad$ Phys. Rev. Lett. 110, 262002 arXiv:1305.1539 [hep-ph]

$\mathrm{Ji}$, Xiangdong (2014), "Parton Physics from Large-Momentum Effective Field Theory," Sci. China Phys. Mech. Astron. 57, 1407-1412,

arXiv:1404.6680 [hep-ph].

Ji, Xiangdong (2017), "Proton Tomography Through Deeply Virtual Compton Scattering," Natl. Sci. Rev. 4 (2), 213-223, arXiv:1605.01114 [hep-ph],

Ji, Xiangdong (2020), "Fundamental Properties of the Proton in Light-Front Zero Modes," arXiv:2003.04478 [hep-ph].

Ji, Xiangdong, Lu-Chang Jin, Feng Yuan, Jian-Hui Zhang, and Yong Zhao (2019a), "Transverse momentum dependent parton quasidistributions," Phys. Rev. D99 (11), 114006. arXiv:1801.05930 [hep-ph]

Ji, Xiangdong, Yizhuang Liu, and Yu-Sheng Liu (2019b), "Transverse-Momentum-Dependent PDFs from LargeMomentum Effective Theory," arXiv:1911.03840 [hep-ph]

Ji, Xiangdong, Yizhuang Liu, and Yu-Sheng Liu (2020a), "TMD soft function from large-momentum effective theory," Nucl. Phys. B 955, 115054, arXiv:1910.11415 [hep-ph]

Ji, Xiangdong, Yizhuang Liu, and Yu-Sheng Liu (in preparation), .

Ji, Xiangdong, Yizhuang Liu, Andreas Schäfer, Wei Wang, Yi-Bo Yang, Jian-Hui Zhang, and Yong Zhao (2020b), "A Hybrid Renormalization Scheme for Quasi LightFront Correlations in Large-Momentum Effective Theory," arXiv:2008.03886 [hep-ph]

Ji, Xiangdong, Yizhuang Liu, and Ismail Zahed (2019c), "Quasiparton distribution functions: Two-dimensional scalar and spinor QCD," Phys. Rev. D99 (5), 054008. arXiv:1807.07528 [hep-ph]

Ji, Xiangdong, Andreas Schäfer, Xiaonu Xiong, and Jian-Hui Zhang (2015a), "One-Loop Matching for Generalized Parton Distributions," Phys. Rev. D92, 014039 , arXiv:1506.00248 [hep-ph]

Ji, Xiangdong, Andreas Schäfer, Feng Yuan, Jian-Hui Zhang, and Yong Zhao (2016), "Spin decomposition of the electron in QED," Phys. Rev. D93 (5), 054013 , arXiv:1511.08817 [hep-ph]

Ji, Xiangdong, Peng Sun, Xiaonu Xiong, and Feng Yuan (2015b), "Soft factor subtraction and transverse momentum dependent parton distributions on the lattice," Phys. Rev. D91, 074009, arXiv:1405.7640 [hep-ph].

Ji, Xiangdong, Xiaonu Xiong, and Feng Yuan (2012), "Proton Spin Structure from Measurable Parton Distributions," Phys. Rev. Lett. 109, 152005, arXiv:1202.2843 [hep-ph].

Ji, Xiangdong, Xiaonu Xiong, and Feng Yuan (2013a), "Probing Parton Orbital Angular Momentum in Longitudinally Polarized Nucleon," Phys. Rev. D88 (1), 014041. arXiv:1207.5221 [hep-ph]

Ji, Xiangdong, and Feng Yuan (2020), "Transverse 
spin sum rule of the proton," Phys. Lett. B 810, 135786 arXiv:2008.04349 [hep-ph]

Ji, Xiangdong, Feng Yuan, and Yong Zhao (2017a), "Hunting the Gluon Orbital Angular Momentum at the Electron-Ion Collider," Phys. Rev. Lett. 118 (19), 192004. arXiv:1612.02438 [hep-ph].

Ji, Xiangdong, Feng Yuan, and Yong Zhao (2020c), "Proton spin after 30 years: what we know and what we don't?" arXiv:2009.01291 [hep-ph]

Ji, Xiangdong, and Jian-Hui Zhang (2015), "Renormalization of quasiparton distribution," Phys. Rev. D92, 034006 arXiv:1505.07699 [hep-ph]

Ji, Xiangdong, Jian-Hui Zhang, and Yong Zhao (2013b), "Physics of the Gluon-Helicity Contribution to Proton Spin," Phys. Rev. Lett. 111, 112002 , arXiv:1304.6708 [hep-ph]

Ji, Xiangdong, Jian-Hui Zhang, and Yong Zhao (2015c), "Justifying the Naive Partonic Sum Rule for Proton Spin," Phys. Lett. B743, 180-183, arXiv:1409.6329 [hep-ph].

Ji, Xiangdong, Jian-Hui Zhang, and Yong Zhao (2017b), "More On Large-Momentum Effective Theory Approach to Parton Physics," Nucl. Phys. B924, 366-376, arXiv:1706.07416 [hep-ph].

Ji, Xiangdong, Jian-Hui Zhang, and Yong Zhao (2018), "Renormalization in Large Momentum Effective Theory of Parton Physics," Phys. Rev. Lett. 120 (11), 112001 arXiv:1706.08962 [hep-ph]

Jia, Shaoyang, and James P. Vary (2019), "Valence structures of light and strange mesons from the basis lightfront quantization framework," in 18th International Conference on Hadron Spectroscopy and Structure (HADRON 2019) Guilin, Guangxi, China, August 16-21, 2019, arXiv:1911.11191 [nucl-th],

Jia, Yu, Shuangran Liang, LiuJi Li, and Xiaonu Xiong (2017), "Solving the Bars-Green equation for moving mesons in two-dimensional QCD," JHEP 11, 151, arXiv:1708.09379 [hep-ph]

Jia, Yu, Shuangran Liang, Xiaonu Xiong, and Rui Yu (2018), "Partonic quasidistributions in two-dimensional QCD," Phys. Rev. D98 (5), 054011, arXiv:1804.04644 [hep-th]

Jia, Yu, and Xiaonu Xiong (2016), "Quasidistribution amplitude of heavy quarkonia," Phys. Rev. D94 (9), 094005. arXiv:1511.04430 [hep-ph].

Joó, Bálint, Joseph Karpie, Kostas Orginos, Anatoly Radyushkin, David Richards, and Savvas Zafeiropoulos (2019a), "Parton Distribution Functions from Ioffe time pseudo-distributions," JHEP 12, 081, arXiv:1908.09771 [hep-lat]

Joó, Bálint, Joseph Karpie, Kostas Orginos, Anatoly V. Radyushkin, David G. Richards, Raza Sabbir Sufian, and Savvas Zafeiropoulos (2019b), "Pion valence structure from Ioffe-time parton pseudodistribution functions," Phys. Rev. D100 (11), 114512 arXiv:1909.08517 [hep-lat]

Joó, Bálint, Joseph Karpie, Kostas Orginos, Anatoly V. Radyushkin, David G. Richards, and Savvas Zafeiropoulos (2020), "Parton Distribution Functions from Ioffe time pseudo-distributions from lattice caclulations; approaching the physical point," arXiv:2004.01687 [hep-lat].

Kang, Zhong-Bo, Alexei Prokudin, Peng Sun, and Feng Yuan (2016), "Extraction of Quark Transversity Distribution and Collins Fragmentation Functions with QCD Evolution," Phys. Rev. D93 (1), 014009 , arXiv:1505.05589 [hep-ph], Kang, Zhong-Bo, and Jian-Wei Qiu (2009), "Evolution of twist-3 multi-parton correlation functions relevant to single transverse-spin asymmetry," Phys. Rev. D79, 016003 , arXiv:0811.3101 [hep-ph]

Karpie, Joseph, Kostas Orginos, Alexander Rothkopf, and Savvas Zafeiropoulos (2019), "Reconstructing parton distribution functions from Ioffe time data: from Bayesian methods to Neural Networks," JHEP 04, 057. arXiv:1901.05408 [hep-lat].

Karpie, Joseph, Kostas Orginos, and Savvas Zafeiropoulos (2018), "Moments of Ioffe time parton distribution functions from non-local matrix elements," JHEP 11, 178, arXiv:1807.10933 [hep-lat].

Kiptily, D V, and M. V. Polyakov (2004), "Genuine twist three contributions to the generalized parton distributions from instantons," Eur. Phys. J. C37, 105-114, arXiv:hep-ph/0212372 [hep-ph].

Kock, Arthur, Yizhuang Liu, and Ismail Zahed (2020), "Pion and Kaon parton distributions in the QCD instanton vacuum," arXiv:2004.01595 [hep-ph]

Kogut, John B, and Davison E. Soper (1970), "Quantum Electrodynamics in the Infinite Momentum Frame," Phys. Rev. D1, 2901-2913.

Konychev, Anton V, and Pavel M. Nadolsky (2006), "Universality of the Collins-SoperSterman nonperturbative function in gauge boson production," $\quad$ Phys. Lett. B633, 710-714 arXiv:hep-ph/0506225 [hep-ph].

Korchemskaya, IA, and G.P. Korchemsky (1992), "On lightlike Wilson loops," Phys. Lett. B 287, 169-175

Korchemsky, GP, and A.V. Radyushkin (1987), "Renormalization of the Wilson Loops Beyond the Leading Order," Nucl. Phys. B 283, 342-364.

Kovchegov, Yuri V (1999), "Small x F(2) structure function of a nucleus including multiple pomeron exchanges," Phys. Rev. D60, 034008, arXiv:hep-ph/9901281 [hep-ph]. Kovchegov, Yuri V, and Eugene Levin (2012), "Quantum chromodynamics at high energy," Camb. Monogr. Part. Phys. Nucl. Phys. Cosmol. 33, 1-350

Kronfeld, Andreas S, and Douglas M. Photiadis (1985), "Phenomenology on the Lattice: Composite Operators in Lattice Gauge Theory," Phys. Rev. D 31, 2939.

Kumericki, Kresimir, Simonetta Liuti, and Herve Moutarde (2016), "GPD phenomenology and DVCS fitting," Eur. Phys. J. A52 (6), 157, arXiv:1602.02763 [hep-ph]

Kuraev, E A, L. N. Lipatov, and Victor S. Fadin (1977), "The Pomeranchuk Singularity in Nonabelian Gauge Theories," Sov. Phys. JETP 45, 199-204, [Zh. Eksp. Teor. Fiz.72,377(1977)].

Lan, Jiangshan, Chandan Mondal, Shaoyang Jia, Xingbo Zhao, and James P. Vary (2019), "Parton Distribution Functions from a Light Front Hamiltonian and QCD Evolution for Light Mesons," Phys. Rev. Lett. 122 (17), 172001. arXiv:1901.11430 [nucl-th]

Landry, F, R. Brock, G. Ladinsky, and C. P. Yuan (2001), "New fits for the nonperturbative parameters in the CSS resummation formalism," Phys. Rev. D63, 013004 , arXiv:hep-ph/9905391 [hep-ph].

Langnau, Alex, and M. Burkardt (1993), "Ultraviolet regularization of light cone Hamiltonian perturbation theory: Application to the anomalous magnetic moment of the electron (g-2) in light cone gauge," Phys. Rev. D47, 3452-3464.

Lübbert, Thomas, Joel Oredsson, and Maximilian Stahlhofen (2016), "Rapidity renormalized TMD soft 
and beam functions at two loops," JHEP 03, 168 arXiv:1602.01829 [hep-ph]

Leader, E, and C. Lorcé (2014), "The angular momentum controversy: What's it all about and does it matter?" Phys. Rept. 541 (3), 163-248, arXiv:1309.4235 [hep-ph].

Lee, RN, A.V. Smirnov, and V.A. Smirnov (2010), "Analytic Results for Massless Three-Loop Form Factors," JHEP 04, 020, arXiv:1001.2887 [hep-ph].

Lenz, F, M. Thies, K. Yazaki, and S. Levit (1991), "Hamiltonian formulation of two-dimensional gauge theories on the light cone," NATO ASI: Hadrons and Hadronic Matter Cargese, France, August 8-18, 1989, Annals Phys. 208, 1-89.

Lepage, G Peter, and Stanley J. Brodsky (1979), "Exclusive Processes in Quantum Chromodynamics: Evolution Equations for Hadronic Wave Functions and the Form-Factors of Mesons," Phys. Lett. 87B, 359-365.

Lepage, G Peter, and Paul B. Mackenzie (1993), "On the viability of lattice perturbation theory," Phys. Rev. D 48, 2250-2264, arXiv:hep-lat/9209022

Li, Hsiang-nan (2016), "Nondipolar Wilson links for quasiparton distribution functions," Phys. Rev. D94 (7), 074036 arXiv:1602.07575 [hep-ph]

Li, Hsiang-nan, and George F. Sterman (1992), "The Perturbative pion form-factor with Sudakov suppression," Nucl. Phys. B381, 129-140

Li, Ye, Duff Neill, and Hua Xing Zhu (2016), “An Exponential Regulator for Rapidity Divergences," Submitted to: Phys. Rev. D arXiv:1604.00392 [hep-ph].

Li, Ye, and Hua Xing Zhu (2017), "Bootstrapping Rapidity Anomalous Dimensions for Transverse-Momentum Resummation," $\quad$ Phys. Rev. Lett. 118 (2), 022004 arXiv:1604.01404 [hep-ph]

Li, Zheng-Yang, Yan-Qing Ma, and Jian-Wei Qiu (2019), "Multiplicative Renormalizability of Operators defining Quasiparton Distributions," $\quad$ Phys. Rev. Lett. 122 (6), 062002 arXiv:1809.01836 [hep-ph]

Li, Zheng-Yang, Yan-Qing Ma, and Jian-Wei Qiu (2020), "Extraction of Next-to-Next-to-Leading-Order PDFs from Lattice QCD Calculations," arXiv:2006.12370 [hep-ph]

Lin, Huey-Wen, Jiunn-Wei Chen, Saul D. Cohen, and Xiangdong Ji (2015), "Flavor Structure of the Nucleon Sea from Lattice QCD," Phys. Rev. D91, 054510 arXiv:1402.1462 [hep-ph]

Lin, Huey-Wen, Jiunn-Wei Chen, Zhouyou Fan, JianHui Zhang, and Rui Zhang (2020), "The ValenceQuark Distribution of the Kaon from Lattice QCD," arXiv:2003.14128 [hep-lat]

Lin, Huey-Wen, Jiunn-Wei Chen, Xiangdong Ji, Luchang Jin, Ruizi Li, Yu-Sheng Liu, Yi-Bo Yang, Jian-Hui Zhang, and Yong Zhao (2018a), "Proton Isovector Helicity Distribution on the Lattice at Physical Pion Mass," Phys. Rev. Lett. 121 (24), 242003. arXiv:1807.07431 [hep-lat]

Lin, Huey-Wen, W. Melnitchouk, Alexei Prokudin, N. Sato, and H. Shows (2018b), "First Monte Carlo Global Analysis of Nucleon Transversity with Lattice QCD Constraints," Phys. Rev. Lett. 120 (15), 152502 arXiv:1710.09858 [hep-ph]

Lin, Huey-Wen, and Rui Zhang (2019), "Lattice finitevolume dependence of the nucleon parton distributions," Phys. Rev. D100 (7), 074502.

Lin, Huey-Wen, et al. (2018c), "Parton distribu- tions and lattice QCD calculations: a community white paper," Prog. Part. Nucl. Phys. 100, 107-160, arXiv:1711.07916 [hep-ph]

Liu, Keh-Fei (2000), "Parton degrees of freedom from the path integral formalism," Phys. Rev. D62, 074501, arXiv:hep-ph/9910306 [hep-ph]

Liu, Keh-Fei (2016), "Parton Distribution Function from the Hadronic Tensor on the Lattice," PoS LATTICE2015, 115, arXiv:1603.07352 [hep-ph]

Liu, Keh-Fei (2017), "Evolution equations for connected and disconnected sea parton distributions," Phys. Rev. D 96 (3), 033001, arXiv:1703.04690 [hep-ph].

Liu, Keh-Fei (2020), "PDF in PDFs from Hadronic Tensor and LaMET," Phys. Rev. D $102(7), 074502$, arXiv:2007.15075 [hep-ph]

Liu, Keh-Fei, and Shao-Jing Dong (1994), "Origin of difference between anti-d and anti-u partons in the nucleon," Phys. Rev. Lett. 72, 1790-1793, arXiv:hep-ph/9306299

Liu, KF, S.J. Dong, Terrence Draper, D. Leinweber, J.H. Sloan, W. Wilcox, and R.M. Woloshyn (1999), "Valence QCD: Connecting QCD to the quark model," Phys. Rev. D 59, 112001, arXiv:hep-ph/9806491

Liu, Wei-Yang, and Jiunn-Wei Chen (2020), "Renormalon Effects in Quasi Parton Distributions," arXiv:2010.06623 [hep-ph]

Liu, Yu-Sheng, Jiunn-Wei Chen, Luchang Jin, Ruizi Li, HueyWen Lin, Yi-Bo Yang, Jian-Hui Zhang, and Yong Zhao (2018), "Nucleon Transversity Distribution at the Physical Pion Mass from Lattice QCD," arXiv:1810.05043 [hep-lat].

Liu, Yu-Sheng, Wei Wang, Ji Xu, Qi-An Zhang, JianHui Zhang, Shuai Zhao, and Yong Zhao (2019a), "Matching generalized parton quasidistributions in the RI/MOM scheme," Phys. Rev. D100 (3), 034006 arXiv:1902.00307 [hep-ph]

Liu, Yu-Sheng, Wei Wang, Ji Xu, Qi-An Zhang, Shuai Zhao, and Yong Zhao (2019b), "Matching the meson quasidistribution amplitude in the RI/MOM scheme," Phys. Rev. D99 (9), 094036, arXiv:1810.10879 [hep-ph]

Liu, Yu-Sheng, et al. (Lattice Parton) (2020), "Unpolarized isovector quark distribution function from lattice QCD: A systematic analysis of renormalization and matching," Phys. Rev. D101 (3), 034020, arXiv:1807.06566 [hep-lat]

Lorce, Cedric, Barbara Pasquini, Xiaonu Xiong, and Feng Yuan (2012), "The quark orbital angular momentum from Wigner distributions and light-cone wave functions," Phys. Rev. D85, 114006, arXiv:1111.4827 [hep-ph].

Luke, Michael E, and Aneesh V. Manohar (1992), "Reparametrization invariance constraints on heavy particle effective field theories," Phys. Lett. B286, 348-354, arXiv:hep-ph/9205228 [hep-ph].

Luo, Ming-Xing, Xing Wang, Xiaofeng Xu, Li Lin Yang, Tong-Zhi Yang, and Hua Xing Zhu (2019), "Transverse Parton Distribution and Fragmentation Functions at NNLO: the Quark Case," JHEP 10,083, arXiv:1908.03831 [hep-ph]

Luo, Ming-xing, Tong-Zhi Yang, Hua Xing Zhu, and Yu Jiao Zhu (2020), "Quark Transverse Parton Distribution at the Next-to-Next-to-Next-toLeading Order," Phys. Rev. Lett. 124 (9), 092001 arXiv:1912.05778 [hep-ph]

Luscher, Martin, and Stefan Schaefer (2011), "Lattice QCD without topology barriers," JHEP 07, 036 arXiv:1105.4749 [hep-lat]

Ma, Yan-Qing, and Jian-Wei Qiu (2018a), "Exploring 
Partonic Structure of Hadrons Using ab initio Lattice QCD Calculations," Phys. Rev. Lett. 120 (2), 022003. arXiv:1709.03018 [hep-ph]

Ma, Yan-Qing, and Jian-Wei Qiu (2018b), "Extracting Parton Distribution Functions from Lattice QCD Calculations," Phys. Rev. D98 (7), 074021, arXiv:1404.6860 [hep-ph]

Ma, Zhi-Lei, Jia-Qing Zhu, and Zhun Lu (2019), "Quasi parton distribution function and quasi generalized parton distribution of the pion meson in a spectator model," arXiv:1912.12816 [hep-ph]

Mannel, Thomas, Winston Roberts, and Zbigniew Ryzak (1992), "A Derivation of the heavy quark effective Lagrangian from QCD," Nucl. Phys. B368, 204-217.

Manohar, Aneesh V (1991), "Polarized parton distribution functions," Phys. Rev. Lett. 66, 289-292

Manohar, Aneesh V, and Iain W. Stewart (2007), "The ZeroBin and Mode Factorization in Quantum Field Theory," Phys. Rev. D76, 074002 arXiv:hep-ph/0605001 [hep-ph].

Manohar, Aneesh V, and Mark B. Wise (2000), "Heavy quark physics," Camb. Monogr. Part. Phys. Nucl. Phys. Cosmol. 10, 1-191.

von Manteuffel, Andreas, Erik Panzer, and Robert M. Schabinger (2020), "Analytic four-loop anomalous dimensions in massless QCD from form factors," arXiv:2002.04617 [hep-ph]

Maris, Pieter, and Craig D. Roberts (2003), "Dyson-Schwinger equations: A Tool for hadron physics," Int. J. Mod. Phys. E12, 297-365. arXiv:nucl-th/0301049 [nucl-th].

Martinelli, G (1999), "Hadronic weak interactions of light quarks," Nucl. Phys. B Proc. Suppl. 73, 58-71, arXiv:hep-lat/9810013.

Martinelli, G, C. Pittori, Christopher T. Sachrajda, M. Testa, and A. Vladikas (1995), "A General method for nonperturbative renormalization of lattice operators," Nucl. Phys. B445, 81-108, arXiv:hep-lat/9411010 [hep-lat].

Martinelli, G, and Christopher T. Sachrajda (1987), "A Lattice Calculation of the Second Moment of the Pion's Distribution Amplitude," Phys. Lett. B 190, 151-156.

McCartor, Gary (1994), "Schwinger model in the light cone representation," Z. Phys. C64, 349-354. arXiv:hep-th/9406094 [hep-th].

de Melo, J P B C, Isthiaq Ahmed, and Kazuo Tsushima (2016), "Parton Distribution in Pseudoscalar Mesons with a Light-Front Constituent Quark Model," Proceedings, 16th International Conference on Hadron Spectroscopy (Hadron 2015): Newport News, Virginia, USA, September 13-18, 2015, AIP Conf. Proc. 1735 (1), 080012 arXiv:1512.07260 [hep-ph]

Messiah, A (1979), QUANTUM MECHANICS. VOL. 2 (GERMAN TRANSLATION).

Miller, Gerald A (2007), "Charge Density of the Neutron," Phys. Rev. Lett. 99, 112001, arXiv:0705.2409 [nucl-th].

Müller, Dieter, D. Robaschik, B. Geyer, F. M. Dittes, and J. Hořejši (1994), "Wave functions, evolution equations and evolution kernels from light ray operators of QCD," Fortsch. Phys. 42, 101-141, arXiv:hep-ph/9812448 [hep-ph]

Moch, S, J.A.M. Vermaseren, and A. Vogt (2005), "Three-loop results for quark and gluon form-factors," Phys. Lett. B 625, 245-252, arXiv:hep-ph/0508055.

Monahan, Chirstopher, and Kostas Orginos (2017), "Quasi parton distributions and the gradient flow," JHEP 03, 116 arXiv:1612.01584 [hep-lat].

Monahan, Christopher (2018a), "Recent Developments in $x$-dependent Structure Calculations," PoS LATTICE2018, 018, arXiv:1811.00678 [hep-lat]

Monahan, Christopher (2018b), "Smeared quasidistributions in perturbation theory," Phys. Rev. D97 (5), 054507 arXiv:1710.04607 [hep-lat].

Mueller, Alfred H (1985), "On the Structure of Infrared Renormalons in Physical Processes at High-Energies," Nucl. Phys. B250, 327-350

Mueller, Alfred H (1994a), "Soft gluons in the infinite momentum wave function and the BFKL pomeron," Nucl. Phys. B415, 373-385

Mueller, Dieter (1994b), "Conformal constraints and the evolution of the nonsinglet meson distribution amplitude," Phys. Rev. D49, 2525-2535.

Mulders, P J, and R. D. Tangerman (1996), "The Complete tree level result up to order $1 / \mathrm{Q}$ for polarized deep inelastic leptoproduction," Nucl. Phys. B461, 197-237, [Erratum: Nucl. Phys.B484,538(1997)], arXiv:hep-ph/9510301 [hep-ph]

Musch, B U, Ph. Hagler, M. Engelhardt, J. W. Negele, and A. Schafer (2012), "Sivers and Boer-Mulders observables from lattice QCD," Phys. Rev. D85, 094510 , arXiv:1111.4249 [hep-lat]

Musch, Bernhard U, Philipp Hagler, John W. Negele, and Andreas Schafer (2011), "Exploring quark transverse momentum distributions with lattice QCD," Phys. Rev. D83, 094507, arXiv:1011.1213 [hep-lat].

Nachtmann, Otto (1973), "Positivity constraints for anomalous dimensions," Nucl. Phys. B63, 237-247.

Nakanishi, Noboru, and Haruichi Yabuki (1977), "Null-Plane Quantization and Haag's Theorem," Lett. Math. Phys. 1, 371-374.

Nakanishi, Noboru, and Koichi Yamawaki (1977), "A Consistent Formulation of the Null-Plane Quantum Field Theory," Nucl. Phys. B122, 15-28.

Nam, Seung-il (2017), "Quasi-distribution amplitudes for pion and kaon via the nonlocal chiralquark model," Mod. Phys. Lett. A32 (39), 1750218. arXiv:1704.03824 [hep-ph]

Nocera, Emanuele R, Richard D. Ball, Stefano Forte, Giovanni Ridolfi, and Juan Rojo (NNPDF) (2014), "A first unbiased global determination of polarized PDFs and their uncertainties," Nucl. Phys. B887, 276-308, arXiv:1406.5539 [hep-ph]

Orginos, Kostas, Anatoly Radyushkin, Joseph Karpie, and Savvas Zafeiropoulos (2017), "Lattice QCD exploration of parton pseudo-distribution functions," Phys. Rev. D96 (9), 094503, arXiv:1706.05373 [hep-ph]

Pauli, Hans Christian, and Stanley J. Brodsky (1985), "Discretized Light Cone Quantization: Solution to a Field Theory in One Space One Time Dimensions," Phys. Rev. D32, 2001.

Penttinen, M, Maxim V. Polyakov, A. G. Shuvaev, and M. Strikman (2000), "DVCS amplitude in the parton model," Phys. Lett. B491, 96-100, arXiv:hep-ph/0006321 [hep-ph].

Perry, Robert J, Avaroth Harindranath, and Kenneth G. Wilson (1990), "Light front Tamm-Dancoff field theory," Phys. Rev. Lett. 65, 2959-2962.

Politzer, H David (1973), "Reliable Perturbative Results for Strong Interactions?" Phys. Rev. Lett. 30, 1346-1349. 
Politzer, H David, and Mark B. Wise (1988), "Leading Logarithms of Heavy Quark Masses in Processes with Light and Heavy Quarks," Phys. Lett. B206, 681-684.

Polyakov, Alexander M (1980), "Gauge Fields as Rings of Glue," Nucl. Phys. B 164, 171-188

Qiu, Jian-wei, and George F. Sterman (1991), "Single transverse spin asymmetries," Phys. Rev. Lett. 67, 2264-2267

Radici, Marco, and Alessandro Bacchetta (2018), "First Extraction of Transversity from a Global Analysis of Electron-Proton and ProtonProton Data," Phys. Rev. Lett. 120 (19), 192001 arXiv:1802.05212 [hep-ph]

Radyushkin, A V (2017a), "Quasi-parton distribution functions, momentum distributions, and pseudo-parton distribution functions," Phys. Rev. D96 (3), 034025. arXiv:1705.01488 [hep-ph]

Radyushkin, A V (2018a), "Quark pseudodistributions at short distances," Phys. Lett. B781, 433-442. arXiv:1710.08813 [hep-ph]

Radyushkin, A V (2019a), "Theory and applications of parton pseudodistributions," arXiv:1912.04244 [hep-ph]

Radyushkin, Anatoly (2017b), "Nonperturbative Evolution of Parton Quasi-Distributions," Phys. Lett. B767, 314-320. arXiv:1612.05170 [hep-ph]

Radyushkin, Anatoly (2017c), "Target Mass Effects in Parton Quasi-Distributions," Phys. Lett. B770, 514-522, arXiv:1702.01726 [hep-ph]

Radyushkin, Anatoly (2018b), "One-loop evolution of parton pseudo-distribution functions on the lattice," Phys. Rev. D98 (1), 014019, arXiv:1801.02427 [hep-ph],

Radyushkin, Anatoly V (2017d), "Pion Distribution Amplitude and Quasi-Distributions," Phys. Rev. D 95 (5), 056020, arXiv:1701.02688 [hep-ph],

Radyushkin, Anatoly V (2019b), "Generalized parton distributions and pseudodistributions," $\quad$ Phys. Rev. D 100 (11), 116011, arXiv:1909.08474 [hep-ph]

Radyushkin, AV (1999), "Double distributions and evolution equations," $\quad$ Phys. Rev. D 59, 014030, arXiv:hep-ph/9805342

Radyushkin, AV (2019c), "Structure of parton quasi-distributions and their moments," Phys. Lett. B 788, 380-387, arXiv:1807.07509 [hep-ph].

Ralston, John P, and Davison E. Soper (1979), "Production of Dimuons from High-Energy Polarized Proton Proton Collisions," Nucl. Phys. B152, 109

Rossi, GC, and M. Testa (2017), "Note on lattice regularization and equal-time correlators for parton distribution functions," Phys. Rev. D 96 (1), 014507, arXiv:1706.04428 [hep-lat]

Rossi, Giancarlo, and Massimo Testa (2018), "Euclidean versus Minkowski short distance," Phys. Rev. D 98 (5), 054028, arXiv:1806.00808 [hep-lat]

Rothe, HJ (1992), Lattice gauge theories: An Introduction, Vol. 43.

Rutherford, E (1919), "Collision of $\alpha$ particles with light atoms. IV. An anomalous effect in nitrogen," Phil. Mag. Ser.6 37, 581-587. [Phil. Mag.90,no.sup1,31(2010)].

Samuel, Stuart (1979), "COLOR ZITTERBEWEGUNG," Nucl. Phys. B 149, 517-524.

Schaefer, Stefan, Rainer Sommer, and Francesco Virotta (ALPHA) (2011), "Critical slowing down and error analysis in lattice QCD simulations," Nucl. Phys. B 845, 93-119.
arXiv:1009.5228 [hep-lat]

Schäfer, Thomas, and Edward V. Shuryak (1998), "Instantons in QCD," Rev. Mod. Phys. 70, 323-426. arXiv:hep-ph/9610451 [hep-ph].

Scimemi, Ignazio, and Alexey Vladimirov (2018a), "Analysis of vector boson production within TMD factorization," Eur. Phys. J. C78 (2), 89, arXiv:1706.01473 [hep-ph].

Scimemi, Ignazio, and Alexey Vladimirov (2018b), "Systematic analysis of double-scale evolution," JHEP 08, 003 , arXiv:1803.11089 [hep-ph]

Scimemi, Ignazio, and Alexey Vladimirov (2019), "Nonperturbative structure of semi-inclusive deep-inelastic and Drell-Yan scattering at small transverse momentum," arXiv:1912.06532 [hep-ph]

Shanahan, Phiala, Michael Wagman, and Yong Zhao (2020), "Collins-Soper Kernel for TMD Evolution from Lattice QCD," arXiv:2003.06063 [hep-lat]

Shanahan, Phiala, Michael L. Wagman, and Yong Zhao (2019), "Nonperturbative renormalization of staple-shaped Wilson line operators in lattice QCD," arXiv:1911.00800 [hep-lat]

Shifman, Mikhail A, and M. B. Voloshin (1987), "On Annihilation of Mesons Built from Heavy and Light Quark and anti-B0 $<\longrightarrow$ B0 Oscillations," Sov. J. Nucl. Phys. 45, 292, [Yad. Fiz.45,463(1987)].

Shugert, Charles, Xiang Gao, Taku Izubichi, Luchang Jin, Christos Kallidonis, Nikhil Karthik, Swagato Mukherjee, Peter Petreczky, Sergey Syritsyn, and Yong Zhao (2020), "Pion valence quark PDF from lattice QCD," in 37th International Symposium on Lattice Field Theory (Lattice 2019) Wuhan, Hubei, China, June 16-22, 2019, arXiv:2001.11650 [hep-lat]

Sivers, Dennis W (1990), "Single Spin Production Asymmetries from the Hard Scattering of Point-Like Constituents," Phys. Rev. D41, 83

Son, Hyeon-Dong, Asli Tandogan, and Maxim V. Polyakov (2019), "Nucleon quasi-Parton Distributions in the large $\mathrm{N}_{c}$ limit," arXiv:1911.01955 [hep-ph].

Srivastava, Prem P, and Stanley J. Brodsky (2001), "Light front quantized QCD in light cone gauge," Phys. Rev. D64, 045006, arXiv:hep-ph/0011372 [hep-ph].

Stefanis, NG (1984), "GAUGE INVARIANT QUARK TWO POINT GREEN'S FUNCTION THROUGH CONNECTOR INSERTION TO O (alpha-s)," Nuovo Cim. A 83, 205

Sterman, George F (1993), An Introduction to quantum field theory (Cambridge University Press).

Stewart, Iain W, and Yong Zhao (2018), "Matching the quasiparton distribution in a momentum subtraction scheme," Phys. Rev. D97 (5), 054512, arXiv:1709.04933 [hep-ph]

Sufian, Raza Sabbir, Colin Egerer, Joseph Karpie, Robert G. Edwards, Bálint Joó, Yan-Qing Ma, Kostas Orginos, JianWei Qiu, and David G. Richards (2020), "Pion Valence Quark Distribution at Large $x$ from Lattice QCD," arXiv:2001.04960 [hep-lat]

Sufian, Raza Sabbir, Joseph Karpie, Colin Egerer, Kostas Orginos, Jian-Wei Qiu, and David G. Richards (2019), "Pion Valence Quark Distribution from Matrix Element Calculated in Lattice QCD," Phys. Rev. D99 (7), 074507. arXiv:1901.03921 [hep-lat]

Sun, Peng, Joshua Isaacson, C. P. Yuan, and Feng Yuan (2018), "Nonperturbative functions for SIDIS and Drell-Yan processes," Int. J. Mod. Phys. A33 (11), 1841006 
arXiv:1406.3073 [hep-ph]

Tanabashi, M, et al. (Particle Data Group) (2018), "Review of Particle Physics," Phys. Rev. D98 (3), 030001.

Tangerman, R D, and P. J. Mulders (1995), "Intrinsic transverse momentum and the polarized Drell-Yan process," Phys. Rev. D51, 3357-3372, arXiv:hep-ph/9403227 [hep-ph]

Thomas, Anthony William, and Wolfram Weise (2001), The Structure of the Nucleon (Wiley, Germany).

Uehara, S, et al. (Belle) (2012), "Measurement of $\gamma \gamma^{*} \rightarrow \pi^{0}$ transition form factor at Belle," Phys. Rev. D86, 092007. arXiv:1205.3249 [hep-ex]

Vary, J P, H. Honkanen, Jun Li, P. Maris, S. J. Brodsky, A. Harindranath, G. F. de Teramond, P. Sternberg, E. G. $\mathrm{Ng}$, and C. Yang (2010), "Hamiltonian light-front field theory in a basis function approach," Phys. Rev. C81, 035205 arXiv:0905.1411 [nucl-th]

Vladimirov, Alexey (2018), "Structure of rapidity divergences in multi-parton scattering soft factors," JHEP 04, 045 arXiv:1707.07606 [hep-ph]

Vladimirov, Alexey A (2017), "Correspondence between Soft and Rapidity Anomalous Dimensions," $\quad$ Phys. Rev. Lett. 118 (6), 062001 arXiv:1610.05791 [hep-ph]

Vladimirov, Alexey A, and Andreas Schäfer (2020), "Transverse momentum dependent factorization for lattice observables," arXiv:2002.07527 [hep-ph]

Wakamatsu, Masashi (2014), "Is gauge-invariant complete decomposition of the nucleon spin possible?" Int. J. Mod. Phys. A29, 1430012 arXiv:1402.4193 [hep-ph]

Wandzura, S, and Frank Wilczek (1977), "Sum Rules for Spin Dependent Electroproduction: Test of Relativistic Constituent Quarks," Phys. Lett. B 72, 195-198.

Wang, Wei, Yu-Ming Wang, Ji Xu, and Shuai Zhao (2019a), " $B$-meson light-cone distribution amplitude from lattice QCD," arXiv:1908.09933 [hep-ph].

Wang, Wei, Jian-Hui Zhang, Shuai Zhao, and Ruilin Zhu (2019b), "Complete matching for quasidistribution functions in large momentum effective theory," Phys. Rev. D100 (7), 074509, arXiv:1904.00978 [hep-ph]

Wang, Wei, and Shuai Zhao (2018), "On the power divergence in quasi gluon distribution function," JHEP 05, 142 arXiv:1712.09247 [hep-ph]

Wang, Wei, Shuai Zhao, and Ruilin Zhu (2018), "Gluon quasidistribution function at one loop," Eur. Phys. J. C78 (2), 147, arXiv:1708.02458 [hep-ph],

Weinberg, Steven (1966), "Dynamics at infinite momentum," Phys. Rev. 150, 1313-1318.

West, Geoffrey B (1975), "Electron Scattering from Atoms, Nuclei and Nucleons," Phys. Rept. 18, 263-323.

Wilson, Kenneth G (1974), "Confinement of Quarks," Phys. Rev. D10, 2445-2459.

Wilson, Kenneth G, Timothy S. Walhout, Avaroth Harindranath, Wei-Min Zhang, Robert J. Perry, and Stanislaw D. Glazek (1994), "Nonperturbative QCD: A Weak coupling treatment on the light front," Phys. Rev. D49, 6720-6766. arXiv:hep-th/9401153 [hep-th].

Wu, J J, W. Kamleh, D. B. Leinweber, R. D. Young, and J. M. Zanotti (2018), "Accessing high-momentum nucleons with dilute stochastic sources," J. Phys. G45 (12), 125102. arXiv:1807.09429 [hep-lat]

Xiong, Xiaonu, Xiangdong Ji, Jian-Hui Zhang, and
Yong Zhao (2014), "One-loop matching for parton distributions: Nonsinglet case," Phys. Rev. D90 (1), 014051. arXiv:1310.7471 [hep-ph],

Xiong, Xiaonu, Thomas Luu, and Ulf-G. Meißner (2017), "Quasi-Parton Distribution Function in Lattice Perturbation Theory," arXiv:1705.00246 [hep-ph].

Xiong, Xiaonu, and Jian-Hui Zhang (2015), "One-loop matching for transversity generalized parton distribution," Phys. Rev. D92 (5), 054037, arXiv:1509.08016 [hep-ph]

$\mathrm{Xu}$, Shu-Sheng, Lei Chang, Craig D. Roberts, and Hong-Shi Zong (2018), "Pion and kaon valence-quark parton quasidistributions," Phys. Rev. D97 (9), 094014. arXiv:1802.09552 [nucl-th]

Yamawaki, Koichi (1998), "Zero mode problem on the light front," in QCD, light cone physics and hadron phenomenology. Proceedings, 10th Nuclear Summer School and Symposium, NuSS'97, Seoul, Korea, June 23-28, 1997, pp. 116199, arXiv:hep-th/9802037 [hep-th]

Yang, Yi-Bo, Raza Sabbir Sufian, Andrei Alexandru, Terrence Draper, Michael J. Glatzmaier, Keh-Fei Liu, and Yong Zhao (2017), "Glue Spin and Helicity in the Proton from Lattice QCD," Phys. Rev. Lett. 118 (10), 102001. arXiv:1609.05937 [hep-ph].

Yoon, Boram, Michael Engelhardt, Rajan Gupta, Tanmoy Bhattacharya, Jeremy R. Green, Bernhard U. Musch, John W. Negele, Andrew V. Pochinsky, Andreas Schäfer, and Sergey N. Syritsyn (2017), "Nucleon Transverse Momentum-dependent Parton Distributions in Lattice QCD: Renormalization Patterns and Discretization Effects," Phys. Rev. D96 (9), 094508. arXiv:1706.03406 [hep-lat].

Zhang, Jian-Hui, Jiunn-Wei Chen, Xiangdong Ji, Luchang Jin, and Huey-Wen Lin (2017), "Pion Distribution Amplitude from Lattice QCD," Phys. Rev. D95 (9), 094514, arXiv:1702.00008 [hep-lat].

Zhang, Jian-Hui, Jiunn-Wei Chen, Luchang Jin, HueyWen Lin, Andreas Schäfer, and Yong Zhao (2019a), "First direct lattice-QCD calculation of the $x$ dependence of the pion parton distribution function," Phys. Rev. D100 (3), 034505, arXiv:1804.01483 [hep-lat]

Zhang, Jian-Hui, Jiunn-Wei Chen, and Christopher Monahan (2018), "Parton distribution functions from reduced Ioffe-time distributions," Phys. Rev. D97 (7), 074508. arXiv:1801.03023 [hep-ph]

Zhang, Jian-Hui, Xiangdong Ji, Andreas Schäfer, Wei Wang, and Shuai Zhao (2019b), "Accessing Gluon Parton Distributions in Large Momentum Effective Theory," Phys. Rev. Lett. 122 (14), 142001. arXiv:1808.10824 [hep-ph]

Zhang, Jian-Hui, Luchang Jin, Huey-Wen Lin, Andreas Schäfer, Peng Sun, Yi-Bo Yang, Rui Zhang, Yong Zhao, and Jiunn-Wei Chen (LP3) (2019c), "Kaon Distribution Amplitude from Lattice QCD and the Flavor SU(3) Symmetry," Nucl. Phys. B939, 429-446, arXiv:1712.10025 [hep-ph]

Zhang, Qi-An, et al. (Lattice Parton) (2020a), "LatticeQCD Calculations of TMD Soft Function Through LargeMomentum Effective Theory," arXiv:2005.14572 [hep-lat].

Zhang, Rui, Carson Honkala, Huey-Wen Lin, and Jiunn-Wei Chen (2020b), "Pion and Kaon Distribution Amplitudes in the Continuum Limit," arXiv:2005.13955 [hep-lat].

Zhang, Rui, Huey-Wen Lin, and Boram Yoon (2020c), "Probing nucleon strange and charm distributions with lattice QCD," arXiv:2005.01124 [hep-lat]. 
$\begin{array}{ccccc}\text { Zhao, } & \text { Yong } & (2019), & \text { "Unraveling } & \text { high- } \\ \text { energy } & \text { hadron } & \text { structures } & \text { with } & \text { lattice }\end{array}$ QCD," Int. J. Mod. Phys. A33 (36), 1830033 arXiv:1812.07192 [hep-ph]

Zhao, Yong (2020), "Theoretical Developments of the LaMET Approach to Parton Physics," PoS LATTICE2019, 267.
Zhao, Yong, Keh-Fei Liu, and Yibo Yang (2016), "Orbital Angular Momentum and Generalized Transverse Momentum Distribution," Phys. Rev. D93 (5), 054006. arXiv:1506.08832 [hep-ph].

Zuazo, JD (2001), Fourier Analysis . Crm Proceedings \& Lecture Notes (American Mathematical Soc.). 